\title{
A New Power System Stabilizer Designed for Grid- Connected Doubly Fed Induction Generator-Based Wind Generation System
}

\author{
by \\ Rahul Kosuru
}

A thesis submitted to the Faculty of Graduate and Postdoctoral Affairs in partial fulfillment of the requirements for the degree of

Master of Applied Science

in

Electrical and Computer Engineering

Carleton University

Ottawa, Ontario

(C) 2014, Rahul Kosuru 


\begin{abstract}
:
The objective of this thesis is to address the stability of a DFIG based wind generation system and improve the system's performance by employing a power system stabilizer (PSS). The PSS signals are applied to the inner current control loops of the Doubly Fed Induction Generator (DFIG) control scheme (voltage oriented control). The performance characteristics of PSS controllers based on the stator voltage and the network frequency, when the system is subjected to grid faults, are investigated via simulations. The system's performance is demonstrated by the small signal stability analysis and the time domain analysis. In the first step, the analysis is carried out without any PSS and the need for a PSS is addressed. In the next step, the system performance improvement witnessed with the use of the PSS, is discussed. It is observed that the network damping and the system's dynamic stability are improved when employing the designed PSS's to the DFIG based wind energy conversion system.
\end{abstract}




\section{Acknowledgements:}

I would like to thank my supervisor Professor Xiaoyu Wang, for giving me this opportunity and guiding me through my master's research. His helpfulness and cheerful personality made our regular discussions fruitful and enjoyable. Under his supervision, I learned how to explore knowledge and conduct research.

The sincere thank you must go to my research group Akshay Kashyap, Shichao Liu, Jian Xiong as well as my friends S.Kiran Reddy, Chaitanya, Revanth and Sajja.B, for their exceptional support during my master's study.

I would like to thank Ms. Anna Lee for her help and guidance in the department. I would also like to thank Nagui Mikhail for his help in the Hydro lab. Thank you to the Department of Electronics, for its support during the whole research.

I also wish to extend my gratitude to my parents, brother and my loving father for their great love, support and caring. 


\section{Contents}

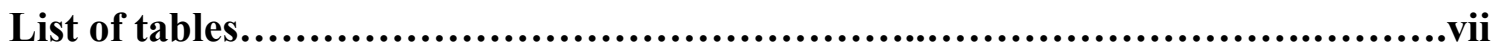

List of figure..........................................................................iii

List of abbreviations....................................................................

List of symbols...........................................................................

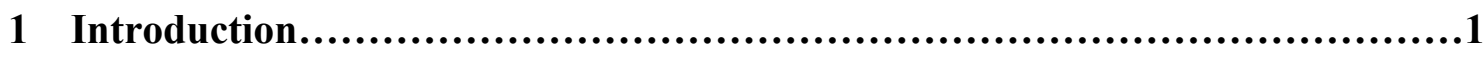

1.1 Renewable Energy Resources........................................... 2

1.2 Wind Energy Conversion System........................................

1.2.1 Stand-alone wind energy conversion system.......................4

1.2.2 Grid integrated wind energy conversion system....................6

1.2.3 On-land and off-shore wind energy conversion system..................8

1.3 A Review of the Design of a Power System Stabilizer..............................9

1.4 Description on the usage of different systems in the research.................12

1.5 Scope and Objective of the Thesis....................................... 15

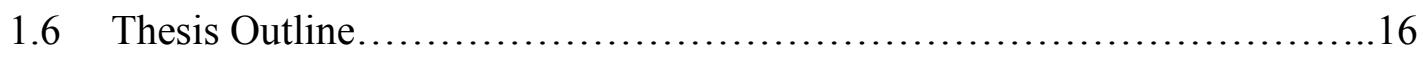

2 Modeling of a DFIG based Wind Energy Generation System......................19

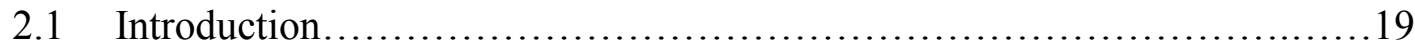

2.2 Classification of Wind Energy Generation System..........................19

2.2.1 Based on the orientation of the axis of rotation.....................20

2.2.2 Based on turbine speed...........................................

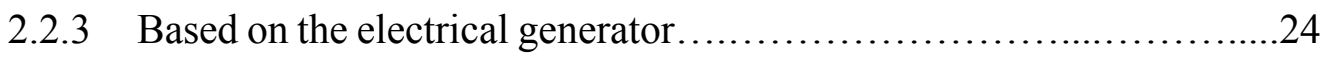

2.3 Doubly Fed Induction Generator based Wind Energy Conversion System.....31

2.4 Modules of the DFIG based Wind Energy Generation System.................33

2.4.1 Aerodynamic and drive train model................................33

2.4.2 Electrical generator (DFIG) model.............................44

2.4.3 Converter model...............................................60

2.4.4 Grid integrated model.........................................62

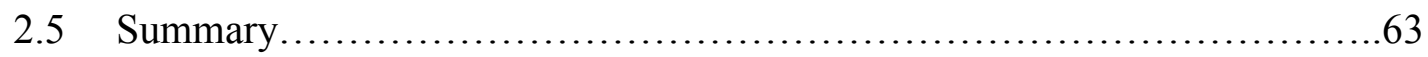

2.6 Summary of large signal equations used for analysis .........................64

3 Controller Design for a DFIG based Wind generation System......................67

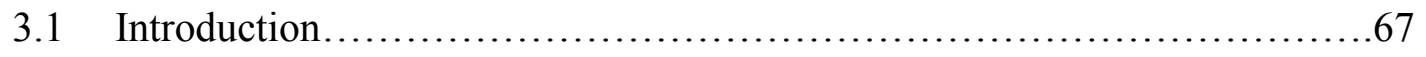


3.2 Design of Mechanical Controller......................................67

3.2.1 Pitch controller design...........................................68

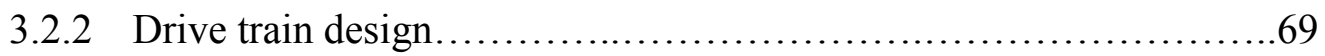

3.3 Design of Electrical Controller...................................... 70

3.3.1 Rotor side converter controller design.............................71

3.3.2 Grid side converter controller design............................... 74

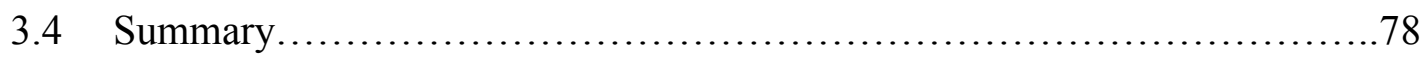

3.5 Summary of large signal equations of controllers from chapter $3 \ldots \ldots \ldots \ldots \ldots . .78$

4 Performance Analysis of a Grid Connected DFIG based Wind Generation

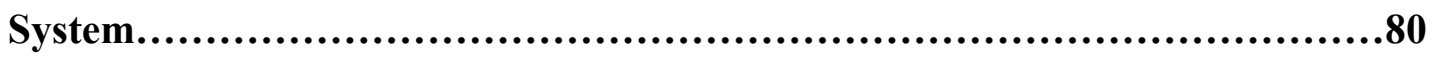

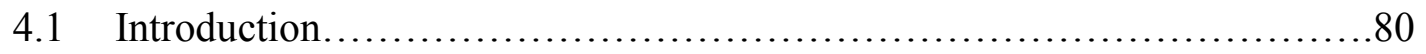

4.2 Small Signal Stability Analysis............................................... 80

4.3 Large Signal Analysis of a DFIG based Wind Generation System............95

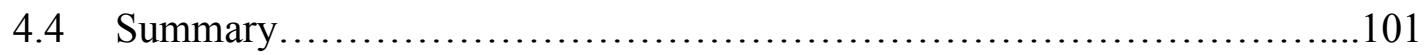

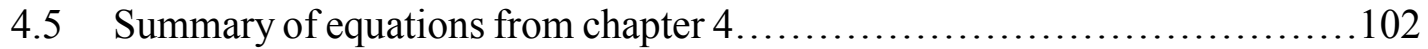

5 Performance Analysis of a Grid Connected DFIG based Wind Generation System with a Power System Stabilizer..........................................106

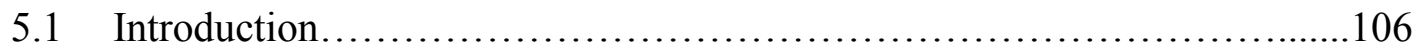

5.2 Power System Stabilizer (PSS) ........................................... 106

5.3 Design and Small Signal Stability Analysis of a DFIG based Wind System with a Power System Stabilizer (PSS) ....................................... 108

5.3.1 Design of voltage PSS............................................109

5.3.2 Modeling of voltage stabilizer...................................110

5.3.3 Small signal stability analysis with voltage PSS ....................111

5.3.4 Modeling of PSS with transformation technique......................114

5.3.5 Small signal model with PSS transformation technique................116

$5.4 \quad$ Large Signal Analysis with PSS..........................................119

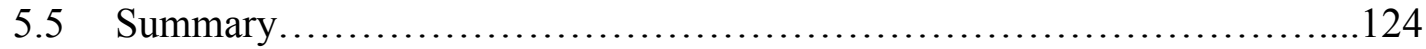

6 Conclusions and Future Work.............................................126

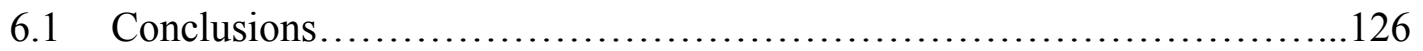

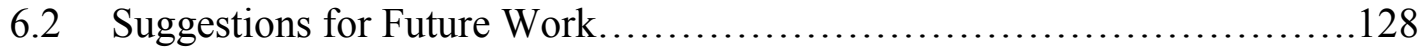


7 Appendices................................................................130

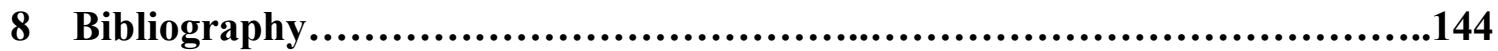




\section{List of tables:}

Table 2.1: Maximum $C_{p}$ and optimal tip speed ratio................................ 38

Table 4.1: Eigenvalues of $(E, A)$ of grid connected system without controller...............84

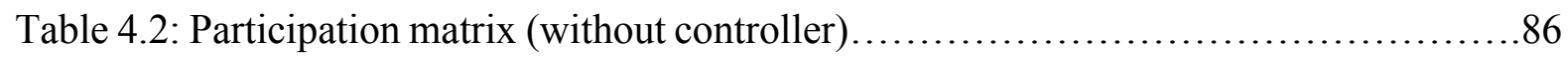

Table 4.3: Eigenvalues of $(E, A)$ and the corresponding state variables...................87

Table 4.4: Eigenvalues of $(E, A)$ for grid integrated DFIG model (with controller).........91

Table 4.5: Participation factors with controllers....................................93

Table 4.6: State variables corresponding to eigenvalues of $(E, A) \ldots \ldots \ldots \ldots \ldots \ldots \ldots \ldots \ldots 4$

Table 5.1: Eigenvalues of $(E, A)$ of grid connected DFIG system with voltage stabilizer...111

Table 5.2: State variables corresponding to eigenvalues............................ 113

Table 5.3: Eigenvalues of $(E, A)$ - grid connected DFIG system with Transformation PSS...117

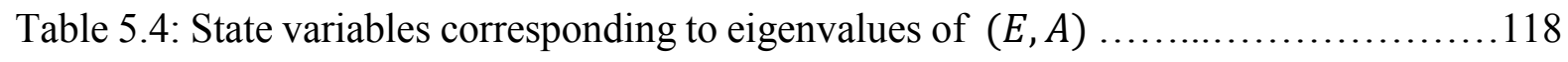




\section{List of figures:}

Figure 1.1: Components of Wind energy conversion system..........................

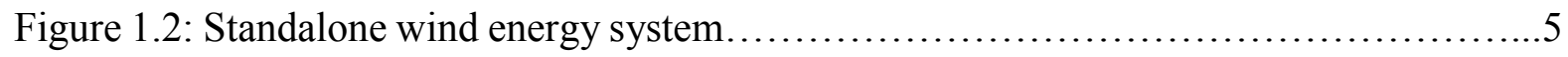

Figure 1.3: Schematic for a grid-integrated wind energy conversion system...............6

Figure 2.1: Schematic of basic wind turbine configurations..........................22

Figure 2.2: Wind turbine system with squirrel cage induction generator (SCIG)...........26

Figure 2.3: Wind turbine system with wound rotor induction generator (WRIG) $\ldots \ldots \ldots \ldots . .27$

Figure 2.4: Wind turbine system with Doubly Fed Induction Generator (DFIG)............28

Figure 2.5(a): Wind turbine system with full power converter generator.................29

Figure 2.5(b): Wind turbine system with full power converter generator (Synchronous

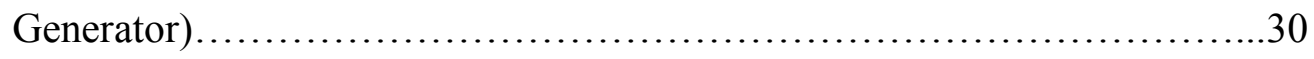

Figure 2.5(c): Wind turbine system with full power converter generator (Permanent

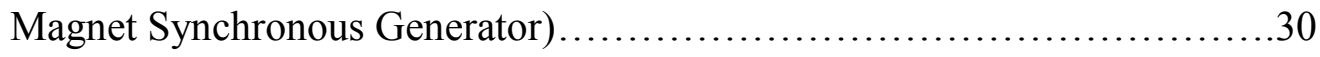

Figure 2.6: Physical model of DFIG based wind generation system...................... 32

Figure 2.7: Theoretical power curve of a wind turbine............................... 37

Figure 2.8: DFIG shaft system, represented by a two-mass model $\ldots \ldots \ldots \ldots \ldots \ldots \ldots \ldots \ldots . .41$

Figure 2.9 (a): Super synchronous mode.....................................47

Figure 2.9 (a): Sub synchronous mode....................................... 47

Figure 2.10: Speed- torque characteristics of DFIG $\ldots \ldots \ldots \ldots \ldots \ldots \ldots \ldots \ldots \ldots \ldots \ldots \ldots \ldots \ldots$

Figure 2.11: Single phase steady state equivalent electric circuit of DFIG.................49

Figure 2.12: DFIG steady state equivalent circuit with different frequencies................50

Figure 2.13: DFIG steady state equivalent circuit referred to stator....................51

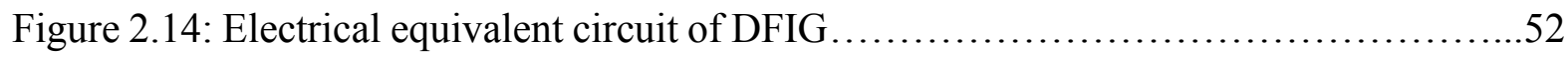


Figure 2.15: two axis reference frame for DFIG...................................56

Figure 2.16 Equivalent circuit of DFIG in synchronously rotating reference frame...........59

Figure 2.17: Converter circuit............................................60

Figure 2.18: Schematic of grid integrated model.................................62

Figure 3.1: Wind turbine model............................................64

Figure 3.2: Pitch controller...............................................65

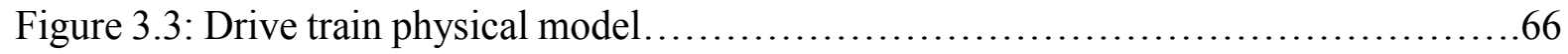

Figure 3.4: Rotor side converter controller block...............................70

Figure 3.5: PLL model........................................................... 72

Figure 3.6: Grid side converter controller.................................... 73

Figure 4.1: Eigen plot without controller....................................... 81

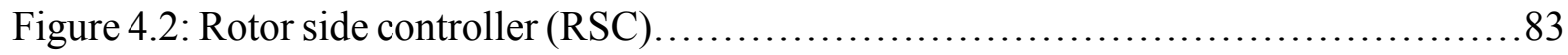

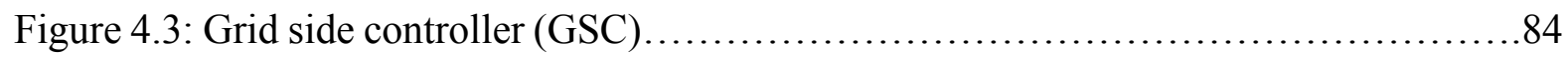

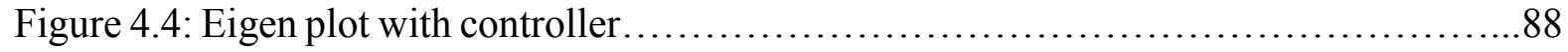

Figure 4.5: Zoomed Eigen plot with controller................................. 88

Figure 4.6: Plot for active power.......................................... 92

Figure 4.7: Reactive power................................................ 93

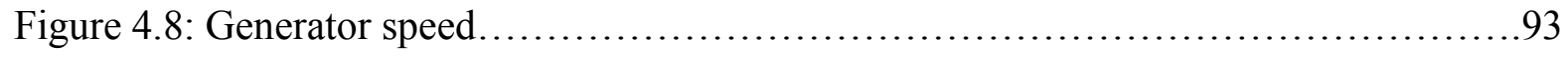

Figure 4.9: DC-link plot................................................. 94

Figure 4.10: Active power during three phase fault at the grid....................... 95

Figure 4.11: Reactive power during three phase fault at the grid end.................... 96

Figure 4.12: DC-Link voltage during three phase fault at the grid end....................96

Figure 4.13: Generator speed during fault condition .............................97

Figure 5.1: General structure of a power system stabilizer.......................... 100 


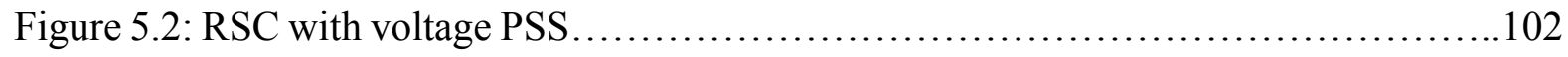

Figure 5.3: Diagram block for voltage PSS .................................. 103

Figure 5.4: Eigen plot with voltage stabilizer.................................. 105

Figure 5.5: RSC with transformation PSS ..................................... 107

Figure 5.6: Block diagram for PSS with transformation technique..................... 108

Figure 5.7: Eigen plot with PSS ........................................... 109

Figure 5.8: (a).Active power and (b).reactive power with and without PSS ...............112

Figure 5.9: (a). DC-link voltage and (b). Generator speed........................... 113

Figure 5.10: (a).Active power and (b).reactive power with and without PSS......................114

Figure 5.11: (a). DC-link voltage and (b). Generator speed with PSS .................. 114

Figure 5.12: (a).Active power and (b).reactive power with and without PSS with fault......115

Figure 5.13: (a). DC-link voltage and (b). Generator speed with PSS during fault...........116

Figure 5.14: (a).Active power and (b).reactive power with and without PSS during fault....116

Figure 5.15: (a). DC-link voltage and (b). Generator speed with PSS during fault...........117

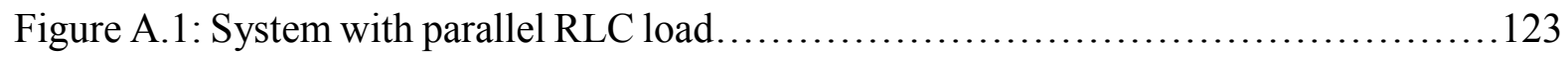




\section{List of abbreviations:}

\begin{tabular}{|c|c|}
\hline DFIG & Doubly Fed Induction Generator \\
\hline WT & Wind Turbine \\
\hline WECS & Wind Energy Conversion System \\
\hline SCIG & Squirrel Cage Induction Generator \\
\hline SG & Synchronous Generator \\
\hline PCC & Point of Common Coupling \\
\hline GSC & Grid Side Converter \\
\hline $\mathrm{RSC}$ & Rotor Side Converter \\
\hline SFOC & Stator Flux Oriented Control \\
\hline SVOC & Stator Voltage Oriented Control \\
\hline PI & Proportional-Integral \\
\hline PLL & Phase-Locked Loop \\
\hline PWM & Pulse Width Modulation \\
\hline IGBT & Insulated Gate Bipolar Transistor \\
\hline VSC & Voltage Source Converter \\
\hline DAE & Differential Algebraic Equation \\
\hline GE & General Electric \\
\hline GEVS & General Electric Voltage Shift \\
\hline GEFS & General Electric Frequency Shift \\
\hline PSS & Power System Stabilizer \\
\hline WRIG & Wound Rotor Induction Generator \\
\hline PMSG & Permanent Magnet Synchronous Generator \\
\hline
\end{tabular}




\section{List of symbols}

\begin{tabular}{|c|c|}
\hline$V_{w}$ & Rated wind speed \\
\hline$\omega_{t}$ & Turbine speed \\
\hline$\theta_{t w}$ & Twist angle of drive train shaft \\
\hline$P_{t}$ & Power extracted by wind turbine \\
\hline$H_{t}$ & Wind turbine inertia constant \\
\hline$K_{t g}$ & Shaft spring constant \\
\hline$D_{t g}$ & Shaft mutual damping \\
\hline$R$ & Blade length \\
\hline$\rho$ & Air density \\
\hline$C_{p}$ & Power coefficient \\
\hline$C_{p}(\lambda, \theta)$ & Performance coefficient \\
\hline$\theta$ & Pitch angle \\
\hline$\lambda$ & Tip speed ratio \\
\hline$C_{\text {pmax }}$ & Maximum value of $C_{p}$ \\
\hline$T_{m}$ & Mechanical torque \\
\hline$T_{e}$ & Electrical Torque \\
\hline$\omega_{s}$ & Synchronous speed \\
\hline$\omega_{r}$ & Rotor speed \\
\hline$S$ & Slip of the generator \\
\hline$P_{S}$ & Generator stator active power \\
\hline$P_{r}$ & Generator rotor active power \\
\hline$Q_{s}$ & Generator stator reactive power \\
\hline$Q_{r}$ & Generator rotor reactive power \\
\hline$H_{g}$ & Generator inertia constant \\
\hline$p$ & No of pairs of poles \\
\hline$v_{d s}$ & Direct component of stator voltage \\
\hline
\end{tabular}




$\begin{array}{ll}v_{q s} & \text { Quadrature component of stator voltage } \\ v_{d r} & \text { Direct component of rotor voltage } \\ v_{q r} & \text { Quadrature component of rotor voltage } \\ i_{d s} & \text { Direct component of stator current } \\ i_{q s} & \text { Quadrature component of stator current } \\ i_{d r} & \text { Direct component of rotor current } \\ i_{q r} & \text { Quadrature component of rotor current } \\ \psi_{d s} & \text { Direct component of stator flux } \\ \psi_{q s} & \text { Quadrature component of stator flux } \\ \psi_{d r} & \text { Direct component of rotor flux } \\ \psi_{q r} & \text { Quadrature component of rotor flux } \\ r_{s} & \text { Stator resistance } \\ r_{r} & \text { Rotor resistance } \\ L_{l s} & \text { Stator inductance } \\ L_{l r} & \text { Rotor inductance } \\ L_{m} & \text { Mutual inductance } \\ V_{d c} & \text { DC-link voltage } \\ C & \text { DC-link capacitance } \\ v_{d g} & \text { Direct component of grid side converter voltage } \\ v_{q g} & \text { Quadrature component of grid side converter voltage } \\ i_{d g} & \text { Direct component of grid side converter current } \\ i_{q g} & \text { Quadrature component of grid side converter current } \\ \omega_{b a s e} & \text { Electrical base speed } \\ \omega_{p l l} & \text { Network frequency acquired by PLL } \\ K_{p} ; K_{i} & \text { Proportional and integral gains } \\ Q_{s r e f} & \text { Reactive power reference } \\ K_{p} & \text { Gain of PSS with voltage as input } \\ T_{w} & \text { Time constant of washout filter } \\ & \end{array}$




\section{Chapter 1}

\section{Introduction:}

Electric power generation plays a significant role in industrializing any society. The generation of energy is increasing from day to day to meet the demand of the growing industries and the individual house loads. Conventionally, electric power, which is produced at the generating station, is transmitted to individual loads and industries through a transmission network. This transmission network consists of a transmission system, a distribution system and many electrical sources and loads. Generally at the electric power generation station, energy that is available in one of the natural forms, is converted to electric form and is transmitted to the grid. The natural forms of energy that are available at the generating station can be divided into two categories:

- Non-renewable energy resources: Coal, fuel oil, natural gas and nuclear energy.

- Renewable energy resources: Wind, hydro, solar, bio, geothermal and ocean energy.

The demand for electric power generation cannot be met by using only non-renewable energy resources, as they are depleting quickly and moreover they are limited. Electric power, which is generated by using fossil fuels, has many disadvantages such as an impact on the environment, a high cost of energy generation and limited reserves of fuels. One of the best solutions to overcome these issues, as well as meet the energy demand is, the integration of renewable energy resources to power grid.

While integrating any renewable energy resource to an existing power grid, one of the fundamental requirements is to be considered is, the supply of a stable power to the grid. Therefore, a proper design and analysis are needed when integrating the renewable source to the power grid. 


\subsection{Renewable Energy Resources:}

From the global energy consumption statistics, it can be observed that renewable energy sources contribute a major share of the power supplied to the grid; together, renewable and non-renewable sources are able to meet the demand. As of 2012, if the total amount of global energy consumption taken was $100 \%$ the share of the non-renewable sources was $81 \%$, with fossil fuels contributing $78.4 \%$ and nuclear power $2.6 \%$. The share of the renewable energy sources was a total of $19 \%$. By the end of 2013 , the non-renewable energy consumption was reduced to $77.9 \%$ while the renewable share was increased to $22.1 \%$. In terms of renewable energy sources used globally, hydropower contributes the main share with $16.4 \%$, and wind energy generation occupies the second place with $2.9 \%$ share, next bio-power delivers $1.8 \%$, solar PV with $0.7 \%$, and finally, ocean energy $0.4 \%$ [1]. When G7 countries are taken into account, Canada is considered as the world leader in the use of renewable energy sources for electric power generation [3]. As of 2012, the total power generated in Canada was 616TWh (terawatt-hours). In this, hydro was contributing $61.2 \%$, nuclear $14.5 \%$, coal $9.5 \%$, gas/oil $11.5 \%$, and other renewable sources $3.3 \%$. In other words, the contribution from renewable sources totalled $64.5 \%$ of Canada's total power supply, placing Canada in the first place among G7 countries [2]. Hydro's current installed capacity is 75,403MW. By the end of 2013 the global installed capacity of solar PV was $139,000 \mathrm{MW}$ and 1,210MW in Canada, in part because of the largest solar PV farm located at the Sarnia Solar Project 2, with a capacity of $60 \mathrm{MW}$. At the end of 2013, the global capacity of wind power was $318,105 \mathrm{MW}$ while in Canada it amounted to 7,803MW [2]. In Canada as and globally wind generation is the second most important renewable energy source after hydro. The main aim of this research is to focus on the design and analysis of a grid connected wind generation system. 


\subsection{Wind Energy Conversion System:}

It can be observed from the global energy statistics, as well as from the Canadian energy consumption statistics, that wind energy plays an important role in renewable energy power generation.

A wind energy conversion system works by capturing, the power of the wind in the wind turbine, with the help of the aerodynamic blades, this energy then converted to mechanical energy. The rotating mechanical energy is then fed to an electrical generator that converts the mechanical energy to electrical energy. In order to supply a standard speed to the generator, a gear system is usually located between the aerodynamic model and the generator model to maintain the speed between the low speed shaft (the turbine shaft) and the high speed shaft (the generator shaft). The electrical power produced by the generator is then supplied to the desired load, or to the grid, through a power electronic converter system and transformer. The arrangement of the wind system is indicated in the block diagram shown below.

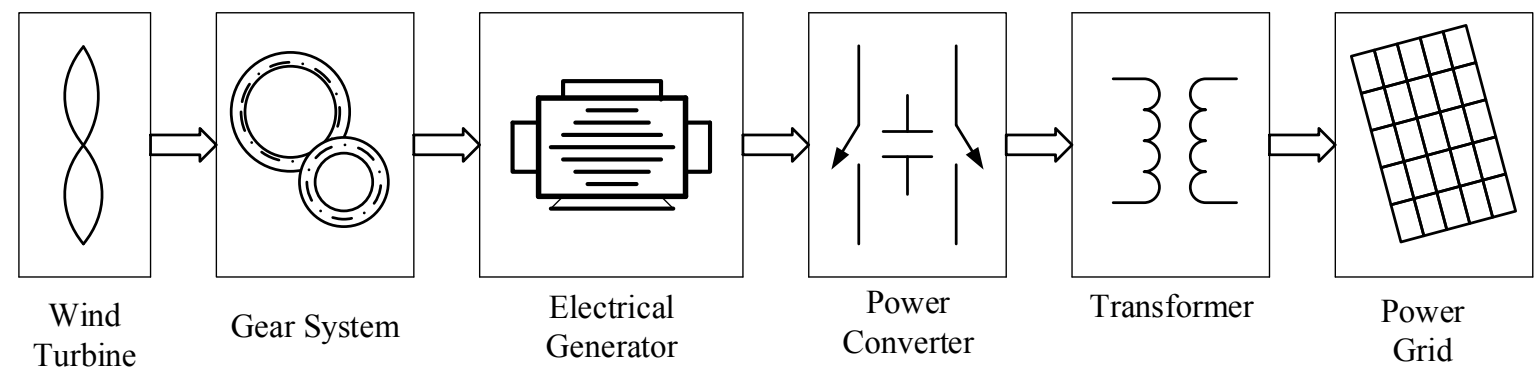

Figure 1.1: Components of wind energy conversion system [8]

As discussed above the components used in a wind generation system can be seen in Figure 1.1. 
The power generated by using a wind energy conversion system can be utilized directly by the load or it can be supplied to the grid. The wind generating system can be placed on-land or it can be located off-shore. Based on the location of the wind generation system and the type of the system connected, wind energy system can be classified as follows:

- Stand-alone wind energy conversion system

- Grid integrated wind energy conversion system

- On-land and off-shore wind energy conversion system.

\subsubsection{Stand-alone wind energy conversion system:}

Most of the primary wind energy systems, developed are stand-alone wind energy systems used to generate electric power. These wind farms are not directly connected to the main electric grid. Stand-alone wind farms small in size and are developed to feed the load to which they are directly connected; these wind farms do not have any impact on the grid. A standalone wind farm is very useful when the location of the load is very far from the generating station. In this case transmission costs can be reduced to minimum, and the load can be fed directly by using the stand-alone system. In general most of the stand-alone wind farms are equipped with an additional energy storage devices such as batteries, since the desired wind speed cannot be reached consistently. Stand-alone wind farms are very useful for storing electricity in the batteries, generating power for remote area lighting, and emergency lighting, and generating power to run the electric motors used to pump water for irrigation and cattle. As the use of the stand-alone wind farm is small, its installed capacity is also low that that of grid connected wind energy systems.

A stand-alone wind energy system which is connected to an individual load is shown in Figure 1.2. At the load end, the system is connected directly to the load without being connected to 
the grid. Here, the wind turbine, which is driven by wind speed, provides the necessary mechanical energy to the generator through a drive train which can be seen in Figure 1.2.

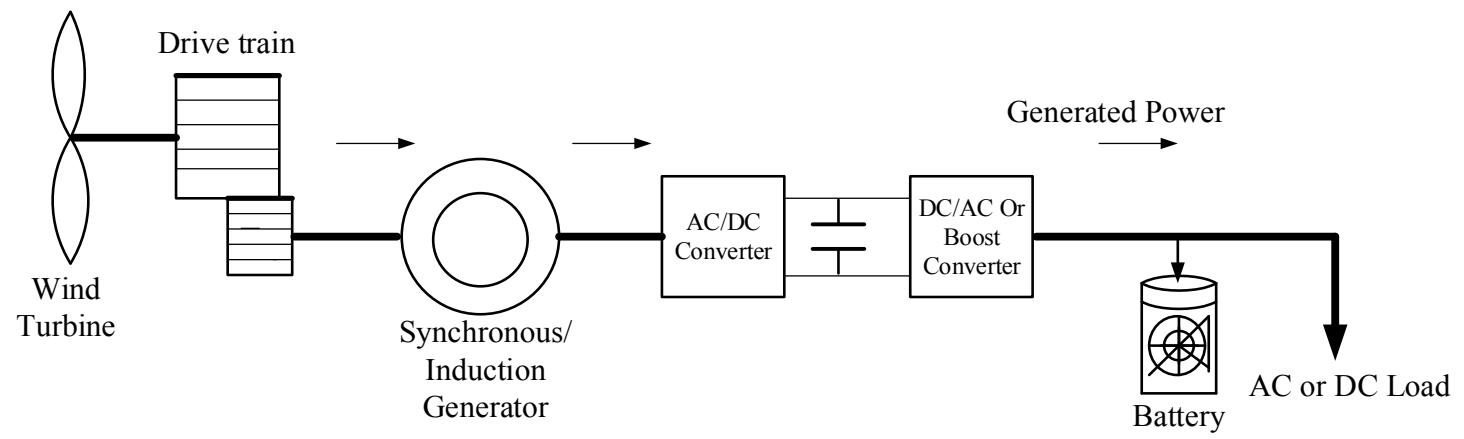

Figure 1.2: Stand -alone wind energy system

The electrical power which is generated is fed to a converter block before being delivered to the load connected at the load end. The battery shown in Figure 1.2 acts as a storage unit that can be used in the case of an emergency, for example when wind speeds are low. The load connected at the load end can be an AC or a DC system. A proper design and control is needed when connecting the wind energy system to the grid. There is much literature on the control and design of stand-alone systems; a new hybrid control system is shown in [4], which is used to meet the load demand in abnormal cases. In [4], the hybrid system is developed by adopting a wind-battery system by controlling the charge of the battery bank using MPPT and the pitch control of the wind energy system. Reference [5] describes the stabilization of a network frequency by employing a flywheel energy storage system (FESS) in an isolated power system (wind energy system). In [6], a super-conducting magnetic energy storage (SMES) system is used to minimize the frequency fluctuations caused by an isolated wind farm connected to the load. In [7], a voltage frequency controller regulates a PMSG based stand-alone wind system. 


\subsubsection{Grid integrated wind energy conversion system}

Grid integrated wind energy conversion system is more prominent than stand-alone wind system. The majority of the wind energy systems currently installed are grid integrated systems. The physical design of these systems is similar to that of stand-alone systems, but at the load end, the system is integrated with the grid. In this scheme, there is no need for an additional energy system such as a battery or capacitor bank. The power generated is directly fed to the grid through a step-up transformer. The general schematic of a grid-integrated system is shown in Figure 1.3.

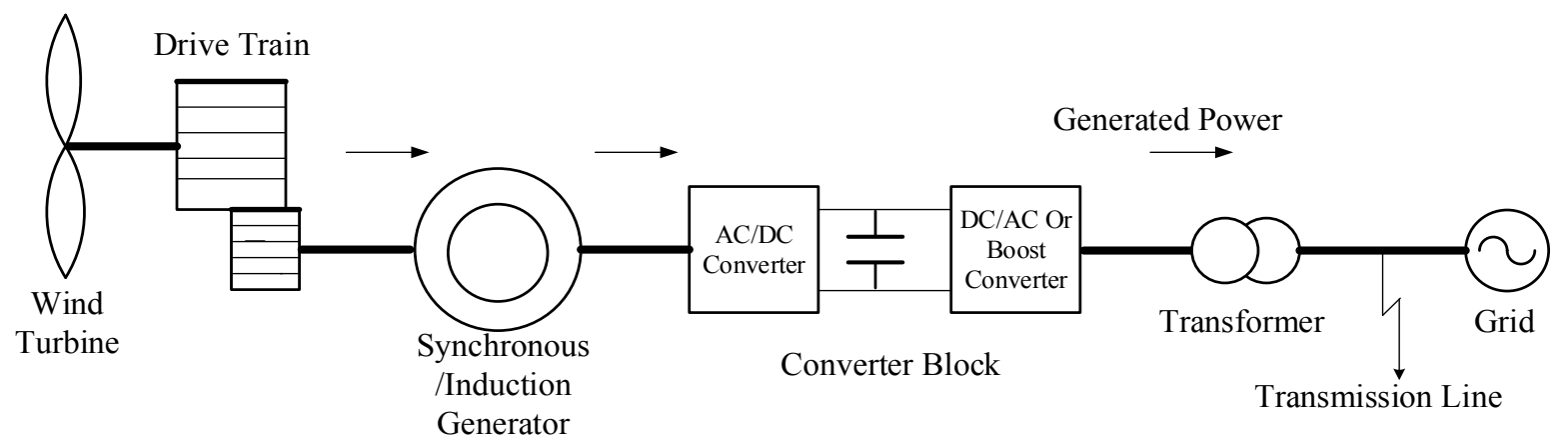

Figure 1.3. Schematic for a grid-integrated wind energy conversion system

The operational functioning of a grid-integrated wind system is similar to that of the standalone wind system discussed in Section 1.2.1, with the exception that here, the system is integrated to the grid. While integrating the system to the grid, care should be taken to design the controller. There is currently much research and analyses taking place to develop control strategies and operational techniques for a grid integrated system. Controllers are designed by considering each component of a wind energy conversion system separately. The components can be observed in Figure 1.1. Design of the controllers can be classified in to two categories,

- Mechanical controller design.

- Electrical controller deign 
When considering the design of the mechanical controller in literature, review [9] deals with the modeling of the mechanical drive train, [10] deals with the design of the controller by considering wind shear and tower shadow, and [11] discusses the design of the controller by considering the cut-in wind speeds. In reference [12], a clear description is investigated by studying aerodynamic measurements. Speed and pitch controllers are developed in [13]. Similarly, literature works [14], [15], [16], [17], [18], [19], and [20], mainly review the pitch of the controller and aerodynamics of wind turbine.

The design of the electrical controller is mainly based on the electrical machine that is used for the generation of electrical power and the power electronic devices. Usually, the electric generators used are induction and synchronous generators. The design of controllers can be classified in to two categories:

- Machine side controller (MSC)

- Grid side controller (GSC)

There is a lot of research taking place to design and improve each controller. Some of the literature that covers the design of controllers for synchronous generators is discussed in this section. Reference [21] proposes a novel control scheme in which MSC is controlled to extract maximum wind power, and GSC is controlled to stabilize the DC-link voltage. In [22], a new control strategy is reviewed to control the active and reactive powers independently. In this scheme, maximum efficiency is achieved by reducing the switching losses. A finite control set-model predictive control (FCS-MPC) strategy is proposed in [23] by eliminating the linear controllers and modulation stages; with this method, switching stages are reduced. References [24] and [25] explain different control strategies to control the reactive power and the frequency control respectively. For induction generator fed wind energy conversion systems, 
unified voltage and pitch angle controller design is discussed in [26]. Reference [27] reviews the control of the stator flux of the generator to maintain and regulate the voltages, while also retaining a constant frequency. Reference [28] suggests a new control scheme for speed and reactive power control of doubly fed induction generators (DFIG) under unbalanced grid voltages. Reference [29] recommends a GSC control of DFIG to improve the power quality of distribution networks. Reference [30] uses capacitor energy storage for constant power control of DFIG. A comparison on control strategies for induction generator fed wind systems is discussed in [31].

\subsubsection{On-land and off-shore wind energy conversion system}

Most of the wind farms developed are on-land wind energy conversion systems. There are several reasons for the development of on-land wind systems, such as the reduction in the maintenance cost and uncomplicated construction and transmission of power to the load centres. The main drawbacks in on-land wind systems, which often lead to the development of offshore wind systems, is the lack of wind resources on the land and the minimal environmental effects such as audible noise and visual impact [32]. Offshore wind systems are more effective and advantageous in densely populated areas. The main disadvantages of offshore wind farms is construction and maintenance costs, as well as the transmission of power to the load centres. In [33], a study was conducted on transmission voltages and concluded that there was a relationship between the switching voltages and the length of the feeders that are energized. Reference [34] worked on a controller design and proposed a method for linear parameter varying modelling and control for an off-shore wind farm.

However the main focus of this research is on on-land wind energy conversion system. When it comes to supplying the power to the grid, whether it be on-land or off-shore wind energy 
conversion systems, the main constraint is supplying a constant power or a stabilized power to the grid. In this thesis a new power system stabilizer is designed for a grid integrated wind energy conversion system.

\subsection{A Review of the Design of a Power System Stabilizer:}

In general any power system network (grid) experiences a lot of disturbances, as there may be a great deal of variations at the load end or at the power generating stations (renewable or nonrenewable). For any system to operate without any disturbances, the critical control issues that need to be considered are power system stability and voltage regulation [35]. The importance of power system stability can be illustrated clearly by viewing [36], which presents a clear description of power system instability as the primary grounds for any major black out. In the present thesis, the necessity and design of a power system stabilizer is emphasized, along with the controllers used to design the DFIG fed wind generation systems. This section offers a review on power system stabilizers by comparing the proposed model with the existing models. A clear description of the design and control of PSS is discussed in Chapter 5.

In a DFIG fed wind generation system the decoupled controllers are used to control the rotor side active power and reactive power, the generator speed, and tracking the maximum power. They also help maintain DC voltage and regulate grid side active and reactive powers. The power system stabilizer can be applied at the output or the input of the controller. In this thesis, the proposed stabilizer is applied at the input of the controller. A power system stabilizer can be employed for any DFIG variable that is influenced by the network oscillations such as rotor speed, stator electrical power, and voltage or network frequency [37]. In this research work two PSS are implemented. One of the PSS is implemented by considering the network frequency, and the second stabiliser is employed by considering the generator's terminal 
voltage. Reference [37] proposes a PSS based on the generator slip signal. The main difference between the proposed model and the model in [37] is that the PSS signal is employed at the DFIG controller and converter interface in [37]. Where as in [38], [39] and [40], the PSS is employed at the input of the active power by taking the rotor speed deviation as the signal for different performance methods. The proposed model is quite different from those in [37]-[40] in the implementation of the controllers and PSS. In the present thesis PSS is employed at the interface of the generated reference signal and the input signal to the inner current control $\operatorname{loop}\left(I_{d r}^{r e f}, I_{d r}^{*}\right.$ or $\left.I_{q r}^{r e f}, I_{q r}^{*}\right)$. Based on [37]-[40] it is reported that the damping of the oscillations in the network fed by a DFIG, can be improved by employing a PSS in the system [41]. The PSS used in [41] and [42] is similar to the PSS proposed in [39] and [40], where a clear study of the optimization of the parameters and the impact of the tuned damping controller under super/sub-synchronous modes of operation are presented. However the main purpose of this research is not to focus on the mode of operation but to investigate the impact of the new PSS on the system's performance. In [41] the damping controller signal is derived from the generator speed $\left(\omega_{r}\right)$ and employed at the rotor phase angle control. Improving stability with a PSS is proposed in [42], which is similar to the aim of this research with the use of a different PSS. From the results and conclusions made in Chapter 5 and Chapter 6, it can be observed that the proposed PSS provides the system with complete damped oscillations. The control strategies that will be discussed in Chapter 3 and the proposed PSS that will be studied in Chapter 5 are very different than those in [37]-[42].

The PSS in [43] is mainly used to damp the electromechanical oscillations and is employed at the voltage quadrature axis, which is fed to the DFIG model. The main difference between the proposed model and [43] remain in the design and the implementation of PSS. However, a 
clear analysis is not presented in [43]. In [44], two stabilizers are employed for a large wind system at the same time and are able to improve the damping of the inter-area power system oscillations, whereas [45] uses a single PSS to improve the damping of inter-area oscillations. The present research has a few similarities to the research conducted in [46], but the design and control of PSS and control strategies are quite different. In [46], the test case is an aggregated model with a large wind farm, which is different from the test case used in this research, and the author successfully improves the network damping capability with the help of two PSS stabilizers. A large wind system is taken as a test case in [47] and successfully obtains a better performance using DFIG PSS. The input signal to the PSS used in [47] is the wind generator power output. References [48] and [49] discussed the oscillations caused by DFIG when integrated in a network that is already fed by a synchronous generator. A coordinated robust control is proposed in [48] by using a power damping oscillator (POD) for DFIG and PSS at a synchronous generator. The IEEE 39 bus system is considered as the test system and a PSS along with an automatic voltage regulator (AVR) are implemented in [49] here the PSS and AVR are implemented at the synchronous generator. References [48] and [49] shows the importance and need of PSS for damping the oscillations caused to the network by the DFIG wind generation system.

The transformation technique and the terminal voltage method used in developing the PSS are similar to the method implemented in [50] and [51]. The major difference is the control strategy and the input signals for the developed PSS are different. Moreover, the proposed system is implemented to a DFIG fed wind generation system.

The main objective of any power system stabilizer is to provide stable power to the grid and to improve the damping of the oscillations. All the power system stabilizers discussed above successfully provide stable power to the grid. Similarly, the proposed model also achieved in 
providing a stable power to the grid with improvements, and major changes such as, the implementation of the stabilizer and the control strategies. From the results of the analysis discussed in Chapters 5 and 6, it can be observed that the proposed model has greater success in supplying a stable power to the grid.

\subsection{Description on the usage of different systems in the research:}

For a clear understanding of different systems used in the research a clear description is provided in this section. The systems different systems used are:

\section{a) Linear system:}

A system is said to be linear if it satisfies the properties of superposition and homogeneity. Superposition principle states that the response of the system to a weighted sum of signals should be equal to the corresponding weighted sum of the outputs of the system to each of the individual input signals. This can be explained by considering two different inputs, $x$ and $y$, in the domain of function $f$ and it can be represented as $f(x+y)=f(x)+f(y)$. The property of homogeneity states that for a given input, $x$, in the domain of the function $f$, and for any real number $a$, and it can be represented as $f(a x)=a f(x)$. General representation of a linear system is as follows:

$$
\dot{x}=A x
$$

\section{b) Nonlinear system:}

Any function that does not satisfy the superposition principle and homogeneity is nonlinear. It can be represented as:

$$
\dot{x}=f(x)
$$

In general the behavior of power system, can be represented as a set of $n$ first order nonlinear ordinary differential equations of the following form [82]: 


$$
\dot{x}_{i}=f_{i}\left(x_{1}, x_{2}, \ldots, x_{n} ; u_{1}, u_{2}, \ldots, u_{r} ; t\right) i=1,2, \ldots, n
$$

Where $n$ is the order of the system and $r$ is the number of inputs. This can be represented in vector-matrix notation as, $\dot{x}_{i}=f(x, u, t) . X$ is refereed as state vector and $x_{i}$ as state variables represented as $x=\left[x_{1}, x_{2} \ldots \ldots x_{n}\right]^{T}$ and $U$ is the vector of input variables given as $U=$ $\left[u_{1}, u_{2} \ldots \ldots u_{r}\right]^{T}$

\section{State variable:}

Any set of $n$ linearly independent system variables may be used to describe the state of the system. These are referred to as state variables; they form a minimal set of dynamic variables that, along with the inputs to the system, provide a complete description of the system behavior. These state variables may be physical quantities in a system such as angle, speed, voltage, or they may be abstract mathematical variables associated with the differential equations describing the dynamics of the system [82].

\section{c) Equilibrium point:}

Equilibrium point can be stated as the point where the system can stay forever without moving. Simply it can be defined as the points where all the derivatives $\dot{x}_{1}, \dot{x}_{2}, \ldots, \dot{x}_{n}$ are simultaneously zero; they define the point on the trajectory with zero velocity [82].

\section{d) Time invariant system:}

A system is said to be time-invariant if the characteristics of the system do not change with time. For a time-invariant system if $y(n)$ is the response of the system to the input $x(n)$, then the response of the system to the input $x(n-k)$ is $y(n-k)$. That is, if the input sequence is shifted by $k$ samples, the generated output sequence is the original sequence shifted by $k$ samples. This can be explained more clearly by taking by considering a linear system. Consider a linear system with $x$ as the state vector of dimension $n$ and $y$ as the output vector of 
dimension $m$ and $u$ is the input vector of dimension $r$, and $A$ is the state or plant matrix of size $n \times n ; B$ is the control or input matrix of size $n \times r ; C$ is the output matrix of size $m \times n$ and finally $D$ is the matrix which define the proportion of input which appears directly in the output, size $m \times r$. For these a linear time invariant system can be expressed as [90]:

$$
\begin{aligned}
\dot{x}(t) & =A x(t)+B u(t) \\
y(t) & =C x(t)+D u(t)
\end{aligned}
$$

\section{e) Time variant system:}

A system is said to be time variant system, if the matrices $A, B, C, D$ consists of any element that is varying with time. This can be made clear by using the state space model of a dynamic system. A dynamic system in state space model can be represented as:

$$
\begin{aligned}
& \dot{x}(t)=A(t) x(t)+B(t) u(t) \\
& y(t)=C(t) x(t)+D(t) u(t)
\end{aligned}
$$

\section{f) Descriptor system:}

A descriptor system can be defined as the system which consists of a mixture of static and dynamic equations. The general representation of a descriptor system is [91]:

$$
E \dot{x}(t)=A x(t)+B u(t)
$$

Equation (1.8), is a representation of a descriptor system. The system used is a linear timetime invariant system. The vector $x(t)$ is an $n$-dimensional descriptor vector and $u(t)$ is an $m$-dimensional input vector. Matrices $E, A, B$ are constant matrices. $E$ and $A$ are size of $n \times n$ and $B$ is $n \times m$.

\section{g) Large signal system:}

In general every power system network is a nonlinear system. The system representing the entire power system network is called as large signal system and analyzing the stability of the 
large signal system is termed as large signal stability. Analyzing large signal stability is a complex problem when compared with the small signal system.

\section{h) Small signal system:}

Linearizing the large signal system around an equilibrium point or operating point to study the system properties or characteristics at that particular operating point is termed as small signal system.

\section{i) Small signal stability:}

Small signal stability is defined as the ability of the power system to maintain synchronism when the system is subjected to small disturbances. Here the disturbance is considered to be small if the equations that describe the resulting response of the system may be linearized for the purpose of analysis [82].

\subsection{Scope and Objective of the Thesis}

Over the past two decades, wind energy has become very popular among renewable energy sources. In wind energy generation techniques, DFIG technology has gained more popularity due to its flexible control strategies and high efficiency of energy transfer. Even though DFIG generation systems have been very popular, there are some issues related to the oscillations and the supply of unstable power when integrated to the grid. Supplying a stable power to the grid is a challenging problem.

In conventional generating plants, where synchronous generators are used, when issues related to power stability arises, a power system stabilizer is used to overcome these stability problems. One of the method that can be implemented to overcome the stability problems is the application of a PSS. In last decade, research has been taking place to implement power system stabilizer for a DFIG fed wind generation system. This work focuses mainly on stability 
analysis and helps understand the advantages in implementing a stabilizer to improve the damping of oscillations when connected to the grid.

The main objectives of this thesis are:

- To establish an adequate mathematical model of a grid integrated DFIG based wind generation system. The developed model is evaluated in Matlab/Simulink environment.

- To conduct a performance analysis of a grid integrated wind generation system without a power system stabilizer and to investigate the characteristics of the developed model.

- To conduct a performance analysis on a grid integrated DFIG based wind system with a selected PSS and present the characteristics of the developed mode by comparing with the model without a PSS.

- To conduct fault studies for the designed wind generation system with power system stabilizers and validate the system's performance.

\subsection{Thesis Outline:}

The research is conducted using analytical and simulation method. The thesis is organized as follows:

The thesis is organized in 6 chapters. The present chapter discusses the importance of renewable energy resources by reviewing the present and future statistics. From the statistics it can be observed that wind energy resources are the most promising and rapidly emerging technology. This chapter discusses about different modes of connecting a wind system to the load. It exposes about the problems related to unstable power supply and how to overcome these issues. In this chapter the necessity of studying the impact of grid integrated wind generation system is emphasized and the importance of a PSS for a DFIG based wind 
generation system is presented. A thorough literature review of power system stabilizers is provided, and finally, the scope and objective of the work carried out in this thesis is revealed. Chapter 2, starts by classifying different wind energy generation systems by presenting the importance and benefits of DFIG wind generation system. A clear description of DFIG based wind generation systems is given. Finally the chapter ends with the presentation of the mathematical modeling of a DFIG based wind generation system. Chapter 2 discloses the mathematical modeling of

a) Aerodynamic and drive train model

b) Modelling of an electrical generator (DFIG)

c) Modeling of the converter

d) Grid integrated model

The models presented in Chapter 2 need to be controlled. Chapter 3 discusses about the control strategies for the models developed. The different control strategies discussed in Chapter 3 are as follows: to control the aerodynamic and drive train model, a mechanical controller is designed, for the electrical generator and converter model, electrical controllers are developed. Finally, to control the grid, a grid controller is presented.

A performance analysis of a DFIG based wind generation system is presented in Chapter 4. First, the analysis is carried out for a large signal detail and average model in Matlab/Simulink. Small signal stability studies and time domain analysis are accomplished by validating the developed model. In this chapter, the entire analysis is carried out without a stabilizer. Its conclusion shows the effect of the oscillations on the network that occurs in the system.

A new power system stabilizer is designed and implemented in Chapter 5, in the presentation of the improvement in damping the oscillations. In Chapter 5, a clear design and description of a power system stabilizer is discussed. Small signal and large signal analyses are carried out 
with a PSS for a DFIG based wind generation system. The effects of the designed PSS are presented in the same chapter. Finally, a fault analysis is executed on the developed system with the two power system stabilizers.

At last, Chapter 6 summarizes the main contributions and conclusions of the thesis. Some possible avenues for future work are also discussed. 


\section{Chapter 2: Modeling of a DFIG Based Wind Energy Generation System}

\subsection{Introduction:}

The main aim of this chapter is to present a clear description and mathematical modeling of the different modules in a DFIG based wind generation system. Before discussing mathematical modeling, the basic concepts of a wind energy generation system, when connected to a utility grid, are presented to ensure a clear understanding of its characteristics and operations. Section 2.2 provides a detailed description of the classification of grid integrated wind energy generation systems, and then presents the importance of the system as well as the factors that lead to the choice of a DFIG as the generator for this thesis. Section 2.3 will offer a literature review of doubly fed induction generators. The mathematical modeling background of all the modules of DFIG based wind generation systems is presented in Section 2.4. Finally, the chapter ends with a summary describing the importance of Chapter 3.

\subsection{Classification of Wind Energy Generation System:}

In wind energy generation systems, wind energy acts as the main source of energy (prime mover), passing through the blades of the wind turbine. The wind speed will cause the blades to start rotating at a certain pace. Generally, the average wind speed is $10-15 \mathrm{~m} / \mathrm{sec}$. The maximum wind speed to which the blades can resist is about $25 \mathrm{~m} / \mathrm{sec}$, beyond which the blades stop rotating, as they can get damaged.

Firstly, the wind speed mainly affects the orientation of the axis of rotation which, in return, affects the generation of electricity. Secondly, the generation of electrical energy depends on wind turbine speed. Thirdly, the classification is made based on the generator used. A clear description of wind system classifications is discussed in the following section.

Wind systems can be classified as follows: 


\subsubsection{Based on orientation of axis of rotation}

2.2.2 Based on turbine speed

2.2.3 Based on electrical generator.

\subsubsection{Based on the orientation of the axis of rotation:}

One of the classifications of wind energy conversion systems is made based on the orientation of axis of rotation; it can also be said that it is based on the constructional design. The distinction is made between the rotors physical design. There are two types of rotors used in wind energy conversion systems [52]:

a) Rotors with a vertical axis of rotation

b) Rotors with a horizontal axis of rotation.

\section{a) Rotors with vertical axis of rotation:}

If the orientation of the spin axis is perpendicular to the ground or the wind turbines in which the blades rotate about a vertical axis, they are termed as vertical axis wind turbines (VAWTs). The main advantage of VATWs is their simple design, which includes the possibility of housing mechanical and electrical components, a gearbox, and a generator at the ground level [52]; this helps neglect the yaw system. For this reason, the installation and maintenance costs

are low. The main disadvantages are: its low tip speed ratio, the limited control on wind speed, the limited control on the output power, and the pitch angle control. These disadvantages are why horizontal axis wind turbines (HAWTs) are often chosen. The other reason for choosing HAWTs is that the geometrical shape of the rotor blades in VAWTs is complicated, which causes many wind turbine manufacturers to design and develop larger horizontal axis wind turbines.

For a better understanding of the differences between VAWTs and HAWTs, the schematic of basic wind turbine configurations is shown in Figure 2.1. From the figure, it can be seen that 
for the VAWT, the generator and the gearbox are placed at ground level. The configuration shown for VAWT is the most common configuration and is called Darrieus turbines [53]. The other types of VAWTs are "H" or "box" turbines; these turbines are not as common as Darrieus turbines.

\section{b) Rotors with horizontal axis of rotation:}

The most common type of wind turbines manufactured are horizontal axis wind turbines (HAWTs). In HAWTs, the turbine's blades rotate around a horizontal axis. The superiority of this design is largely based on the following characteristics [52]:

- The power output and rotor speed can be controlled by using blade pitch control.

- With the help of blade pitch control large wind turbines can be protected against over speed and extreme wind speeds.

- Achievement of higher efficiency by easy tracking of the maximum power point.

- The ability to turn the blades.

- Easy control and usage of power electronic devices.

Because of these advantages, all the wind turbines which are used in present scenario are horizontal axis wind turbines.

Some of the disadvantages are:

- Complex construction and design, like mounting the generator and gear box on a tower.

- High installation and maintenance cost.

For a better understanding of the horizontal axis wind turbines the schematic is shown in Figure 2.1. In General, a horizontal axis wind turbine consists of rotor blades, nacelle which consists of a gearbox, a generator, a control unit and power converters and the mechanical shaft. The physical location of the different components that are discussed above can be seen 
in Figure 2.1. These HAWTs are further classified based on the flow of wind, such as the upwind speed and the down wind speed. A clear description about the classification based on flow of wind is not provided as the topic is mainly related to aerodynamics which is not the main aim of this research work. However the prime focus of this thesis is on the use of horizontal axis wind turbines to develop a wind model which is discussed in the following sections of this chapter.

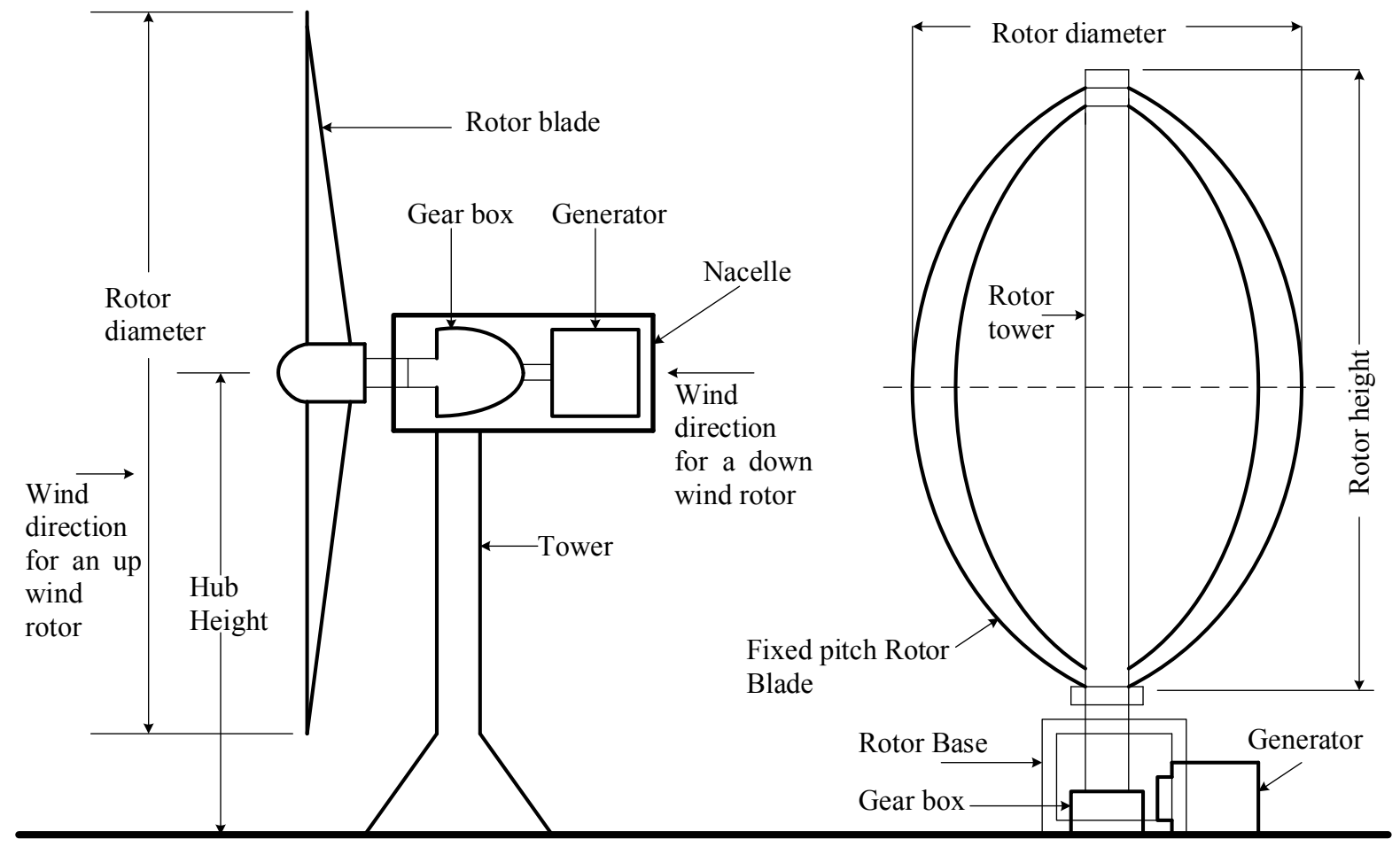

a) Horizontal axis wind turbine(HAWT)

b) Vertical axis wind turbine(VAWT)

Figure 2.1: Schematic of basic wind turbine configurations [53].

From this point of section the wind turbine design and analysis is carried out only for horizontal axis wind turbines.

\subsubsection{Based on turbine speed:}

The next classification of wind turbines is made based on the turbine speed. This classification is very important since the control strategies and the electrical generators that are developed 
for any research depend on the turbine speed. A clear description of the type of the generators that can be developed is presented in Section 2.2.3. The control methods and the generators for wind turbines are developed based on two types of wind turbine speeds. They are [54]:
a) Fixed speed wind turbines and
b) Variable speed wind turbines.

\section{a) Fixed speed wind turbines:}

In a fixed speed wind turbine, the rotor is initially held in a stop position but then accelerates when the brakes are released. The rotor starts rotating with the wind until it hits the predetermined fixed speed. Eventually, when the rotor hits the specified fixed speed, the system is synchronized with the grid through the generator that is used, and the grid holds a constant speed. Technically, a fixed speed wind turbine can be specified as the turbine in which the asynchronous machine is coupled to a fixed frequency electrical network, which rotates at a quasi-fixed mechanical speed, independent of wind speed [54]. In this type of wind turbine, maximum efficiency is achieved at the specified fixed speed. If the wind speed is higher or lower, the efficiency of the specified speed will be reduced. This is one of the main drawbacks; the other disadvantages are high fluctuations in the output power and bad power quality. Because of all these disadvantages, there is great preference for variable speed wind turbines. The main advantages of a fixed speed wind turbine are its low cost and simple structure and design.

\section{b) Variable speed wind turbines:}

The speed wind turbine is simply a technological evolution of a fixed speed wind turbine. In a variable speed wind turbine, the wind speed is matched to the rotor, which eventually allows the best flow of the wind to the rotor and extracts maximum power from the wind flow. Extracting the maximum efficiency and control of from rotor is facilitated by adding a pitch 
controller to the rotor design. Therefore, the design of rotor blades and of control strategies is very complex in variable speed wind turbines. These types of turbines have more control over the output power as well as many advantages when compared to fixed speed wind turbines. Variable speed wind turbine brings the following advantages over fixed speed wind turbine:

- Full control over the output power and a stable power is supplied to the connected grid.

- Can operate even at low wind speeds and sustain for some extent of higher wind speeds.

- Higher efficiency can be achieved by employing maximum power tracking method.

- Less mechanical loads.

In [55], a comparison is made between constant-speed (fixed speed) and variable speed wind turbines. It concludes that variable speed operation gives an improved wind production and a more flexible system, for various wind conditions. With all these advantages, nowadays most of the wind turbines manufactured are variable speed wind turbines.

\subsubsection{Based on the electrical generator:}

In a wind turbine system, the process of converting mechanical energy to electrical energy is part of an electrical system. In an electrical system, the main components are the electrical generator, supervisory control, the power electronic devices, and transformers. In these components, the primary item used for the classification of wind energy systems is the generator. However, the other electrical components are similar in many types of wind energy systems; they are determined based on their generation capabilities and the users' requirements. This section mainly deals with the classification of wind energy systems based on the electrical generators. As discussed in Section 2.2.2, the type of generator will also determine the turbine speed, whether it is a fixed speed wind turbine or a variable speed wind turbine. In general, there two types of generator models are used: synchronous and 
asynchronous generators. Further classification is made based on these two types of generator models. Commercially, there are four types of electrical generators that are used in wind energy generation systems. These four types of generators are derived from the two models mentioned above (synchronous and asynchronous). Based on the type of generator and the grid interface, wind energy systems can be categorised into four types $[56,60]$ :

a) Squirrel cage induction generator or fixed speed system

b) Wound rotor induction generator with variable rotor resistance

c) Doubly fed induction generator

d) Full power converter generator

\section{a) Squirrel cage induction generator (SCIG) or Fixed speed system:}

Usually fixed speed wind turbines are employed with a squirrel cage induction generator and are directly connected to the grid. SCIG are mainly used for smaller wind turbines as they are simple and economical compared to other generators. While using an induction generator, a gearbox is employed as an intermediary between the generator and the turbine speeds. An induction generator consists of a stator and a rotor. In a squirrel cage induction generator, the rotor bars are permanently short circuited, therefore the rotor voltage is zero. A wind turbine system with SCIG is shown in Figure 2.2. From the figure, it can be observed that a gear system is employed between the wind turbine rotor and SCIG; the reason for employing a gearbox is discussed above. The stator is connected to a soft starter and then directly to the grid through a transformer. The two important components that can be seen in Figure 2.2 are the capacitor bank and the soft starter. The reason for employing the capacitor bank is mainly to compensate for the reactive power [57], so the size of the capacitor bank needs to be chosen carefully. A soft starter is employed to mitigate high starting currents and to produce a smooth grid connection [57]. Speeding up the turbine during wind gusts, in order to reduce the 
mechanical stresses, is possible by increasing the rotor resistance of a SCIG, which further leads to an increase in the slip to $2-3 \%$, whereas the usual variation of the slip is $1-2 \%$ [57]. However, the system with SCIG cannot be used for higher power ratings.

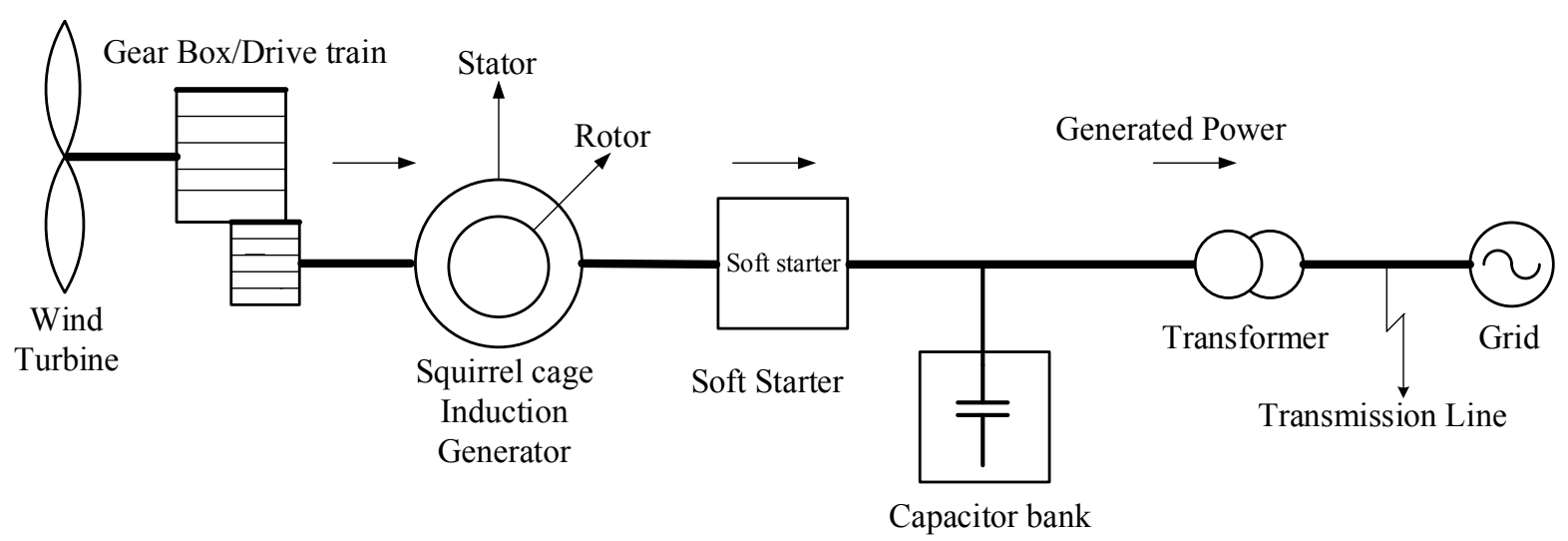

Figure 2.2: Wind turbine system with squirrel cage induction generator (SCIG) $[56,60]$

\section{b) Wound rotor induction generator (WRIG) with variable resistance:}

The next category of wind turbines uses the second type of generator, called a wound rotor induction generator; a fixed speed machine. By employing power electronic converters in a wound rotor induction generator model, we can achieve a much greater control performance [58]. The wind turbine system with WRIG can be seen in Figure 2.3. In the wound rotor induction generator, the stator is directly connected to the grid, as shown in Figure 2.3, but, the wounded rotor winding is connected to a variable resistor via slip rings. This extra resistance, employed in a wound rotor induction generator, can be controlled by using power electronic converters. With this technology, it is possible for the rotor to have configurations such as slip power recovery, the use of cyclo-converters, and rotor resistance chopper control [59]. This is one of the main advantages of WRIG over SCIG. As shown in Figure 2.3, connecting a WRIG wind turbine is similar to connecting one equipped with SCIG. As with SCIG, this solution also consists of a soft starter for a smooth grid connection and capacitor 
bank, to compensate for the reactive power [58]; this is one of the main disadvantages of both technologies (SCIG \& WRIG). Finally, by controlling the rotor resistance, the slip of the machine can be changed to $2-10 \%[57,60]$, through which the generator output can be controlled, and the wound rotor induction generator has a large slip range compared to that of the squirrel cage induction generator. WRIG is preferred for both fixed and variable speed wind turbines, despite the fact that it has limited variable speed control when it is employed for variable wind turbines.

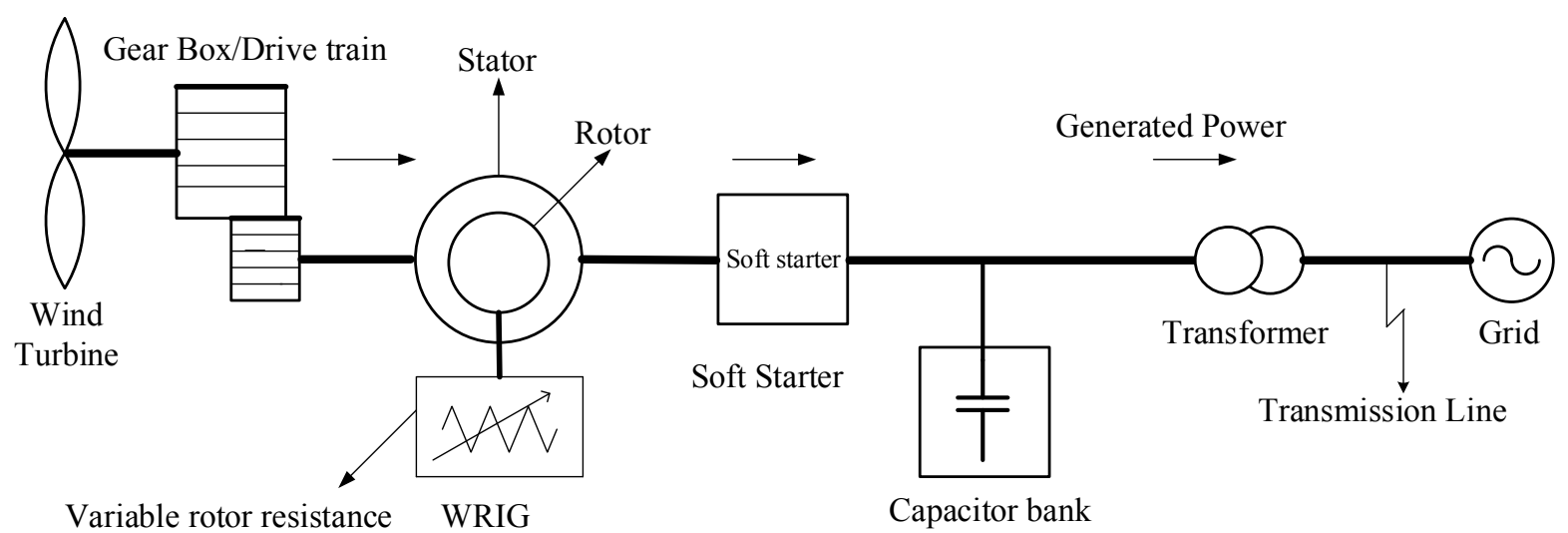

Figure 2.3: Wind turbine system with wound rotor induction generator (WRIG) [56, 60]

\section{c) Doubly fed induction generator (DFIG):}

The third type of generator, based on which a wind turbine system is categorized, is the doubly fed induction generator (DFIG). DFIG is considered one of the best solutions for variable speed wind turbines and large units. Usually, wind turbines with DFIG are employed with power electronic converters. The constructional features of DFIG are similar to those of WRIG, except that in DFIG, the rotor winding of WRIG is connected to the grid through an AC-DC-AC converter. Accessibility to the rotor winding is possible with the use of slip rings and brushes, applied in the rotor circuit design. With the help of power electronic converters, there is control over the rotor speed, which is the main reason for classifying DFIG as a 
variable speed asynchronous generator. By being able to control the speed of DFIG, it is possible to produce power at maximum efficiency, based on the available wind resources. A variable speed wind turbine equipped with DFIG and power electronic converters connected to the grid is shown in Figure 2.4. It can be observed that the stator of DFIG is directly connected to the grid, whereas the rotor is connected to the grid by using a back to back power electronic converter. This research work mainly presents DFIG. A clear description of a DFIG based wind generation system is given in Section 2.3.

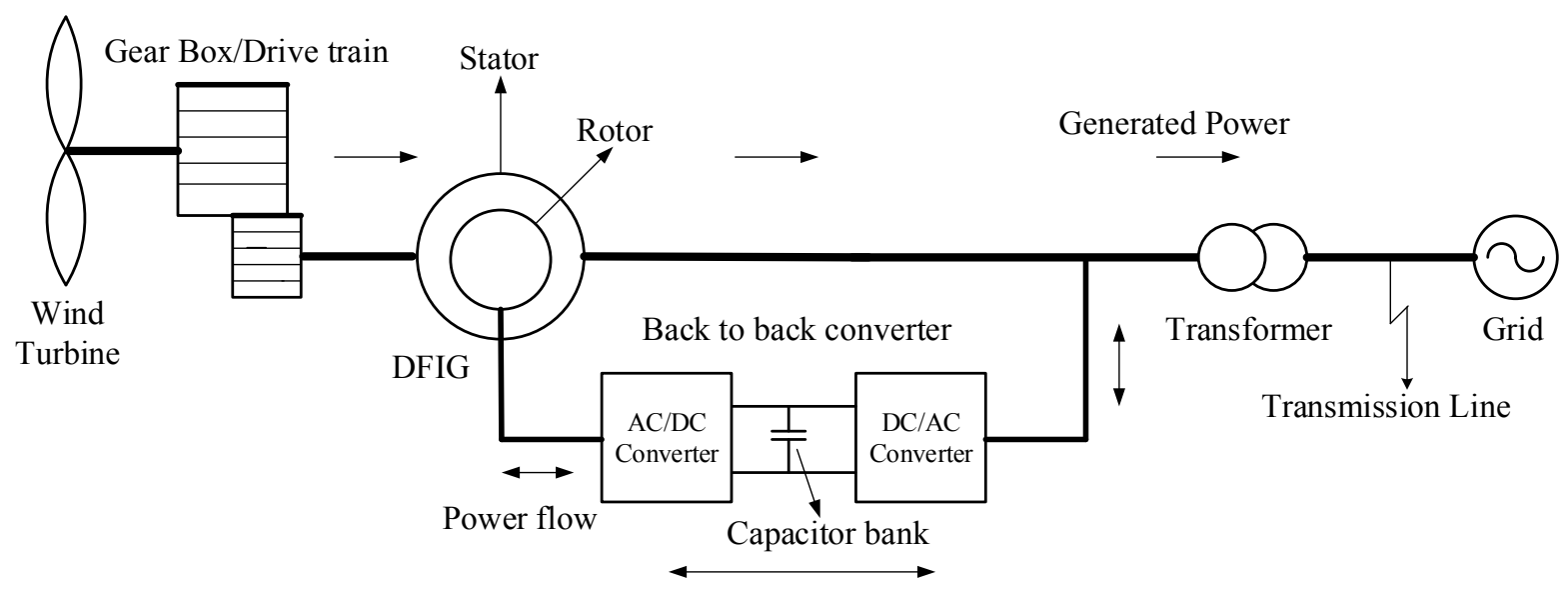

Figure 2.4: Wind turbine system with Doubly Fed Induction Generator (DFIG) [56, 60]

\section{d) Full power converter generator:}

The last type of generator on which the wind turbine system is classified, is the fully converted synchronous generator, or the fully converted squirrel cage induction generator (SCIG). In this configuration, the total power is interchanged between the wind system and the grid through a power electronic converter system, whereas in some of the systems it can be transmitted to the grid directly. [57]. By introducing power electronic converters, there is more control over the electrical machine, and the technical performance also increases. However, there are losses during the power conversion in the power electronic devices. The use of the electrical generator totally depends on the rating of the wind turbine. For larger ratings of wind turbines, 
synchronous generators are suitable, whereas for smaller ratings, squirrel cage induction generators are preferred. The reason is that synchronous generators will operate at a unity power factor whereas SCIG requires reactive power compensation or capacitor banks. In this type of wind system, the drive train/gearbox is optional and totally dependent on the type of generator used. If the generator is a synchronous generator, then a gearbox is not necessary, but if the generator is SCIG, the gearbox is a necessity; this can be observed from [57] and [60]. With induction machines, the wind turbine system cannot be operated without a gearbox because of the extreme excitation losses due to the large air gap [57], whereas in a synchronous generator, the field excitation is provided by a small power electronic converter which is connected externally, or by using a permanent magnet synchronous generator. Therefore in this case, the excitation is given externally, which helps eliminate the need for the gearbox [61]. This explains why the term for the systems using synchronous generators is direct driven synchronous generators in wind systems.

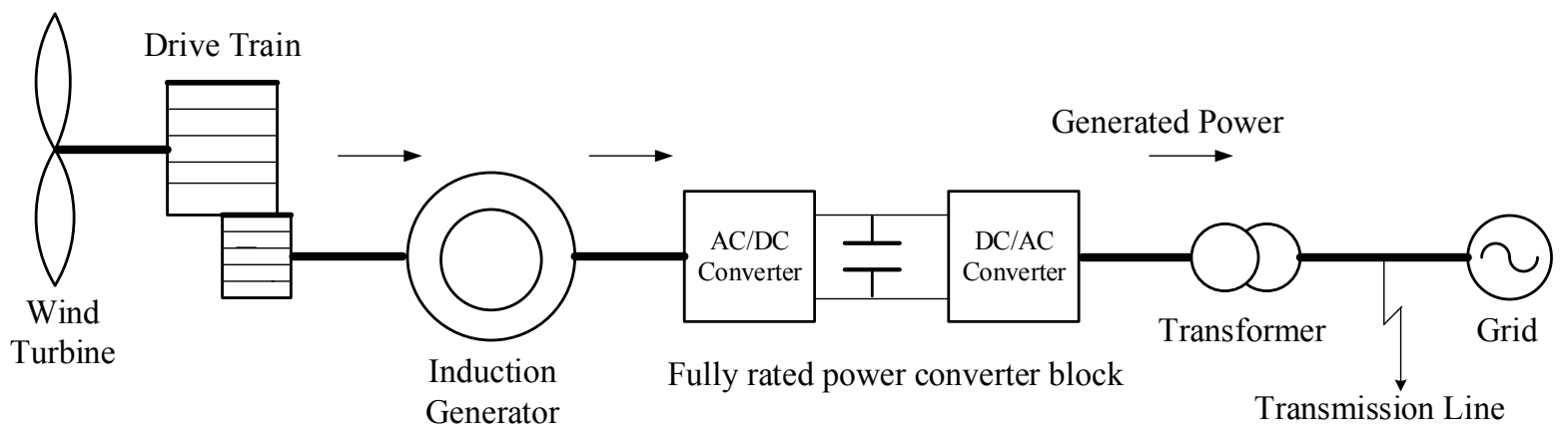

Figure 2.5(a): Wind turbine system with full power converter generator (Induction Generator) $[56,60]$

The Figure 2.5(a), shows the configuration of a wind system with a fully rated power converter. It can be observed that the system is equipped with an induction generator; this can be a SCIG or a WRIG. In this case, a gearbox is employed with the system, whereas Figure 2.5(b) and 
Figure 2.5(c) show the configuration of a wind system with a synchronous generator and with a permanent magnet synchronous generator. It can be recognized that in Figure 2.5(b), the synchronous generator is excited by using the power electronic converter externally, while in Figure $2.5(\mathrm{c})$, it is excited by a permanent magnet, as the generator is a permanent magnet synchronous generator.

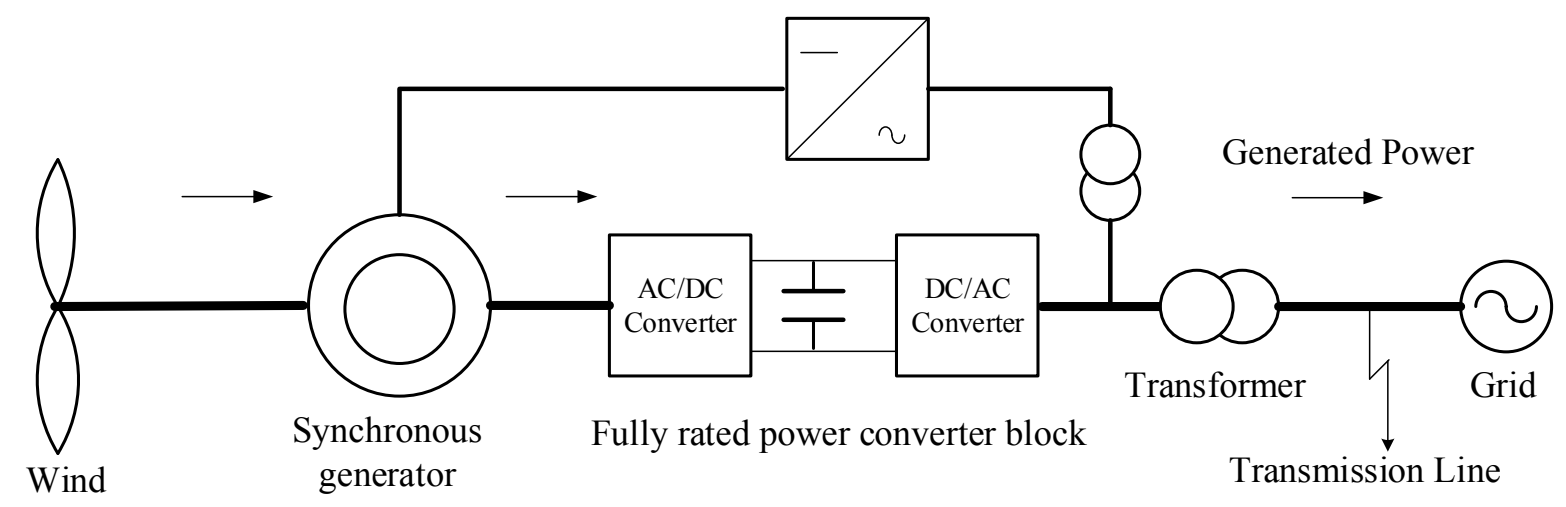

Turbine

Figure 2.5(b): Wind turbine system with full power converter generator (Synchronous

Generator) [56]

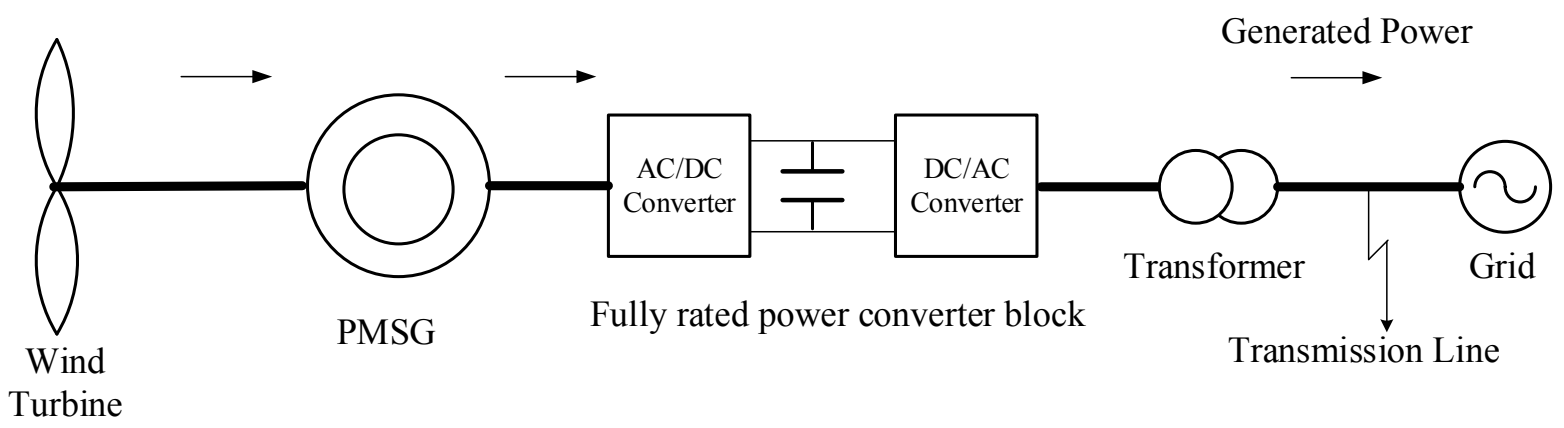

Figure 2.5(c): Wind turbine system with full power converter generator (Permanent Magnet Synchronous Generator) $[56,61]$

Active power and reactive power are controlled by the power electronic converters which are separating the electrical generator and the electrical grid. Thus the control strategies for all the configurations of Figure 2.5 are same. Over the last decade, there is much tremendous move 
in industries in manufacturing variable speed wind turbines, especially there is much demand for DFIG and full converter models [62]. The main reasons for variable speed wind turbines to attract over fixed speed wind turbines include reduced mechanical stresses, increased power control, controllability [62] and operating at unity power factor. For large wind farms type (c) and type (d) are promising technologies and they both have their own advantages and disadvantages over each other.

However in this thesis the analysis is carried out on DFIG based wind generation system.

\subsection{Doubly Fed Induction Generator based Wind Energy Conversion System:}

As discussed above for the variable wind generation system, the best technologies available are using DFIG or a fully converted generator that employs a synchronous generator. In order to reduce the losses in power electronic devices and be efficient, the best method is the DFIG technology. Much research has been done to develop models for the DFIG wind generation system. Reference [63] is used to develop the basic configuration of a DFIG based wind generation system. The main focus of this thesis is DFIG based wind generation system. In this section, a clear description of the DFIG based wind generation system is presented, and the modeling of the system is explored in Section 2.4. For a clear understanding of the physical model of the DFIG wind system, the complete model implemented in this thesis is shown in Figure 2.6. It can be observed from Figure 2.6, that the DFIG stator is directly connected to the gird, while the rotor is connected to the gird via an AC-DC-AC converter. DFIG based wind generation systems have different modules, as shown in Figure 2.6. A clear description of the modules is given in Section 2.4. Here, a general description of the working model is discussed. Initially, wind speed allows for the rotation of the wind turbine, which produces the 
mechanical energy. By using mechanical energy, the shaft starts rotating and provides the required mechanical energy to the generator. Finally, the generator converts the mechanical energy developed into electrical energy and then, it is transmitted and distributed to the grid. In order to deliver the rated power to the grid, the electrical energy generated is transmitted through different stages such as power electronic converters and transformers.

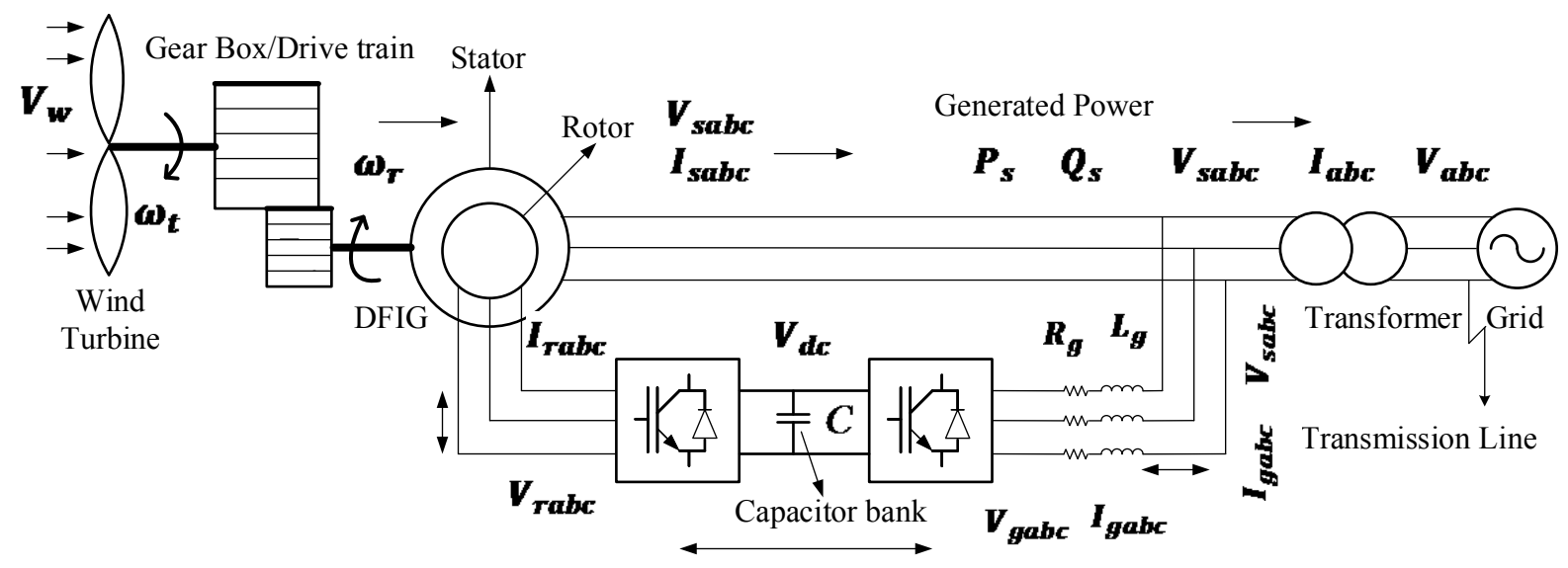

Figure 2.6: Physical model of DFIG based wind generation system.

DFIG based wind generation system can be categorized into two models:

a) Mechanical model and

b) Electrical model

The main aim of a mechanical model is to develop the required mechanical energy for the generator. The mechanical energy is developed by utilizing the kinetic energy available in the wind. In order to develop this energy, the different components that are needed are (1) Wind turbines, (2) Gearbox and (3) Pitch controller. So, the mechanical part consists of the aerodynamic model of the wind system with a gearbox. The function of the electrical model is to generate the required amount of electrical energy to the grid. The different electrical components that are involved in the electrical model are (1) Electrical generator, (2) Power electronic converter and (3) Grid integration model. Based on the components discussed 
above, the entire wind system can be subcategorized into four modules. A clear description of these modules is presented in the next section.

\subsection{Modules of the DFIG based Wind Generation System:}

From Figure 2.6, DFIG based wind generation system can be categorized in to four modules:

2.4.1 Aerodynamic and Drive train model

2.4.2 Electrical generator (DFIG) model

\subsubsection{Converter model}

\subsubsection{Gird integrated model}

\subsubsection{Aerodynamic and Drive train model:}

The main components in aerodynamic and drive train model are the turbine and the drive train. The turbine consists of the rotor blades, the hub, the yaw bearing and the pitching actuators.

\section{The rotor model:}

The blades and the hub together form the rotor. The rotor blades of the turbine converts the available kinetic energy in the wind into mechanical energy. The blade length and number of blades determine the rating of the wind turbine. Generally for a power generation wind turbine system two or three blades are used. In most of the cases three blade wind turbines are used and they are dominant in the present market. The reason for choosing and the advantages of three blade wind turbines are [64]:

- The rotor moment of inertia is easier to understand and it is better to handle than the rotor moment of inertia in a two-bladed turbine.

- Lower noise level and better visual aesthetics than two-bladed wind turbine.

The hub is attached to the low speed shaft of the wind turbine. The yaw bearing is a large cam wheel which is mounted onto the tower. The yaw bearing helps the nacelle of the wind turbine 
to rotate with the rotor against the wind. This yaw bearing uses electrical motors for turning the nacelle. Pitch controller tells the yaw motor when to turn the nacelle.

While modeling wind turbine for electrical power system analysis, most of the mechanical elemental analysis is avoided and kinetic energy captured is represented with a simple equation. With the given wind speed and turbine conditions the aerodynamic model can be represented with equation (2.1). It can be defined as the power contained in the form of kinetic energy in the wind crossing the wind turbine at a speed $V_{w}$, of surface $A$, is expressed as in [54] i.e., $P_{w}=0.5 \rho A V_{w}^{3}$. This equation is obtained as follows [52]. The kinetic energy equation for mass of air $m$ moving at an average speed of $V_{w}$ is expressed as $E=0.5 m V_{w}^{2}$. The power available is the rate of change of energy $P_{w}=\frac{d}{d t}(E)=0.5 \dot{m} V_{w}^{2}$. Mass flow with the air density $\rho$ through an area $A$ is given as $\dot{m}=\rho A V_{w}$. Finally the available wind power is expressed as $P_{w}=0.5 \rho A V_{w}^{3}$.

The wind turbine can use only a part of that power so the wind power extracted by the turbine is given as $P_{t}=P_{w} C_{p}$. Where $C_{p}$ is the power coefficient of wind power that is extracted from wind turbine and it is defined as the ratio between the mechanical power extracted by the wind turbine converter and that of the undisturbed air stream, it can be expressed as $C_{p}=$ $\frac{\text { Extracted power }}{\text { Power in wind }}$. In power system studies as the main concentration is on electrical systems, $C_{p}$ has been developed as a function of tip speed ratio $\lambda$ and the pitch angle $\theta$ [13]. Tip speed ratio $\lambda$ is defined as the ratio of the blade tip linear speed to the wind speed available. It determines the function of available power extracted from the wind turbine rotor. Tip speed ratio $\lambda$ is expressed as $\lambda=\left(\omega_{t} R\right) /\left(V_{w}\right)$, in this expression $\omega_{t}$ is wind turbine rotor angular speed and $R$ is the radius of the blade. In equation (2.1), $A$ is the swept area of the wind turbine 
rotor which is expressed as $A=\pi R^{2}\left[m^{2}\right]$. Finally power extracted from wind turbine is given as $[13,19-21,30,31,47,52,61$, and 63$]$ :

$$
P_{t}=0.5 \rho A V_{w}^{3} C_{p}(\lambda, \theta)
$$

In this equation $P_{t}$ is the mechanical power extracted from the air flow by the wind turbine and it is the function of air density $\rho\left[\mathrm{kg} / \mathrm{m}^{3}\right]$, wind velocity $V_{w}$ in $[\mathrm{m} / \mathrm{s}]$ turbine rotor blade radius $R$ in $[m]$ and power coefficient $C_{p}$.

\section{$C_{p}$ Model:}

In general $C_{p}$ model is a dynamic model. But in this thesis the dynamics of $C_{p}$ model are neglected as they are more related to turbine efficiency and mechanical studies, however the overall dynamic behavior of the wind turbine-generator model would not change. In most of the cases $C_{p}$ curves are provided by manufactures and it is concluded that the power curves of individual wind turbines show a high degree of similarity and a general numerical approximation can be used [65].

For power system a general expression for $C_{p}(\lambda, \theta)$ is given as [65-69]:

$$
C_{p}(\lambda, \theta)=c_{1}\left(\frac{c_{2}}{\lambda+c_{8} \theta}-\frac{c_{2} c_{9}}{\theta^{3}+1}-c_{3} \theta-c_{4} \theta^{c_{5}}-c_{6}\right) \exp \left(\frac{-c_{7}}{\lambda+c_{8} \theta}+\frac{c_{7} c_{9}}{\theta^{3}+1}\right)+C_{10} \lambda
$$

In $C_{p}$ model $\lambda$ is the tip speed ratio and $\theta[\mathrm{deg}]$ is the pitch angle. Tip speed ratio is defined as

$$
\lambda=\left(\omega_{t} R\right) /\left(V_{w}\right)
$$

In plotting for $C_{p}$ model, [68] and [69] used the curve fitting formula. However the power curves have great similarity in all the references discussed above.

$$
C_{p}(\lambda, \theta)=\sum_{i=0}^{4} \sum_{j=0}^{4} \alpha_{i, j} \theta^{i} \lambda^{j}
$$

It is proven in [68] and [69] that this curve fitting approximation provides a good approximation for choosing the range of tip speed ratio $(\lambda)$. Tip speed ratio is one of the 
important parameter as it determines the range of boundaries for wind speed. In [68] tip speed ratio range is approximated as $2<\lambda<13$. The meaning of these boundaries is that, value of $\lambda$ beyond the lower and higher boundaries represent very high and low wind speeds, respectively, that were outside the continuous operating region of the wind turbine generator $[68,69]$.

\section{Betz limit:}

The above discussed $C_{p}$ and the operating regions determine the operating characteristics of any wind turbine. The maximum achievable value of $C_{p}$ is 0.593 known as Betz limit and it determines the maximum amount of wind energy that can be extracted by a wind turbine form the available wind [70] or in other words maximum efficiency of any wind turbine is 0.593 .

\section{Operating region}

Operating region characteristics of wind turbine can be explained clearly by using the power curve. Generally the power curve of a wind turbine is defined as the graph that indicates range of the electrical power output for a given or designed turbine at different wind speeds [71]. Theoretical power curve of a wind turbine is shown in Figure 2.7 [71]: The three operating regions are
(a) Cut-in wind speed,
(b) Rated wind speed and
(c) Cut-out wind speed.

(a) Cut-in Wind speed:

Cut-in wind speed is defined as the wind speed at which usable wind power is generated. The wind turbines which are manufactured are designed to start running at wind speed around 3 $5 \mathrm{~m} / \mathrm{s}$ [71]. This wind speed is called as cut-in wind speed. The machine do not produce any 
power below this wind speed as the energy in the wind is not sufficient to overcome the inertia of the wind turbine rotor.

(b) Rated Wind speed:

The wind speed at which rated power is achieved is called as rated wind speed [71]. Usually the rated wind speed for megawatt size turbine is given as $12-15 \mathrm{~m} / \mathrm{s}$ [71]. The power above the rated wind speed is maintained at a constant level.

(c) Cut-out Wind speed:

The high wind speeds at which the wind turbine is shut down or programmed to stop is called as cut-out wind speeds. Usually this speed is taken as $25 \mathrm{~m} / \mathrm{s}$ [71]. The turbine is shutdown beyond the cut-out wind speeds as to protect or prevent the turbine from damaging.

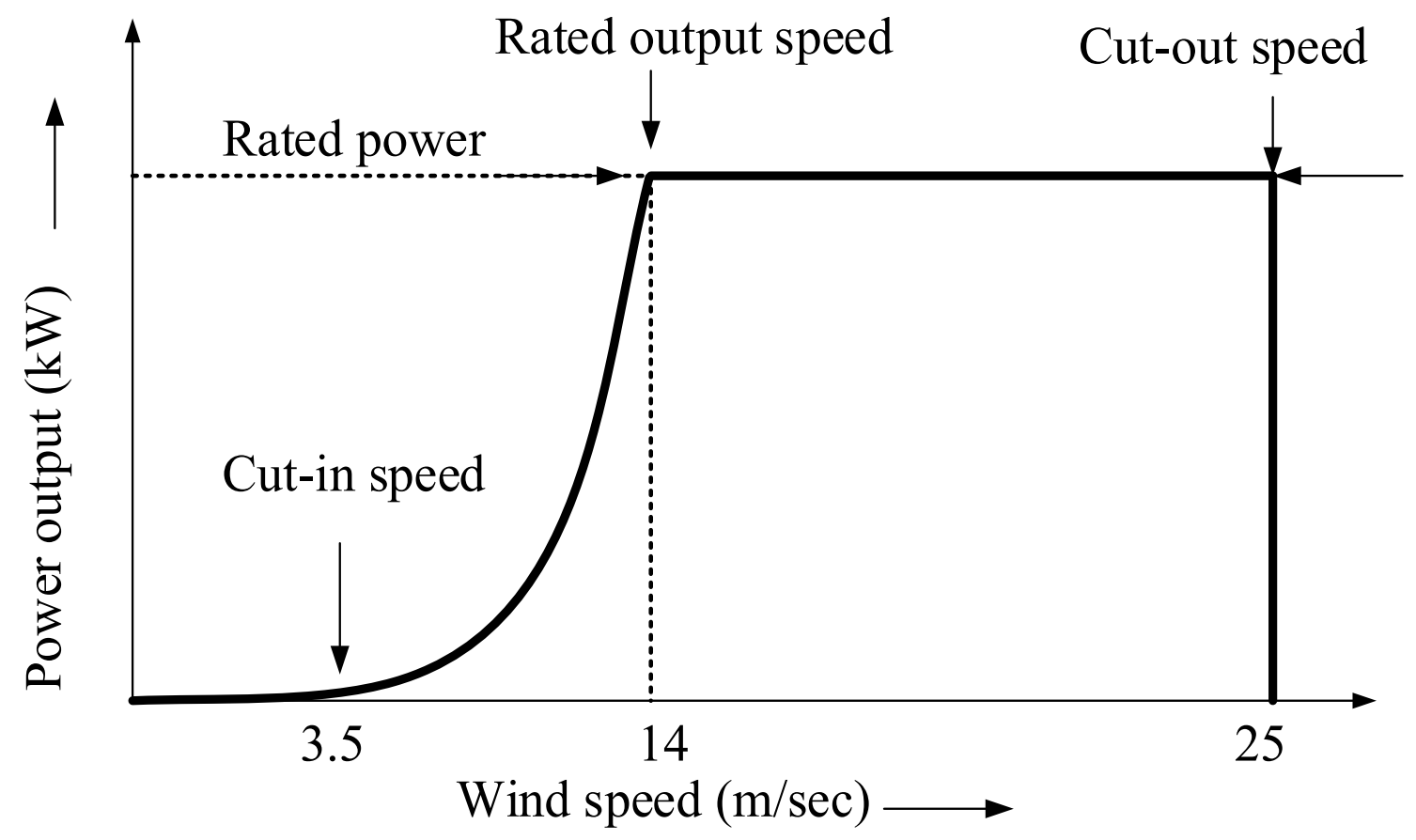

Figure 2.7: Theoretical power curve of a wind turbine [71].

As discussed above the maximum efficiency or the maximum power coefficient is given as 0.593, which is Betz limit. In present market no wind turbine has been designed which is 
capable of exceeding the Betz limit [70]. In practical most of the wind turbines which are designed are able to achieve $75 \%-80 \%$ of Betz limit.

$C_{\text {pmax }}$ is obtained when the blade pitch angle $\theta$ reaches zero. Optimal tip speed ratio $\lambda_{\text {optimal }}$ is defined as the tip speed ratio value obtained at $\theta=0$.

A survey is made on the above discussed references for maximum $C_{p}$ and $\lambda_{\text {optimal }}$ and the following table is obtained:

Table 2.1: Maximum $C_{p}$ and optimal tip speed ratio

\begin{tabular}{|c|c|c|}
\hline Reference & $C_{\text {pmax }}$ & $\lambda_{\text {optimal }}$ \\
\hline$[13]$ and [66] & 0.4382 & 6.325 \\
\hline$[65]$ - constant speed & 0.4906 & 8.7622 \\
\hline$[65]-$ Variable speed & 0.4412 & 7.2064 \\
\hline$[68]$ and [69] & 0.48 & 8.1 \\
\hline Matlab [72] & 0.5 & 9.9 \\
\hline
\end{tabular}

Finally a complete aerodynamic model is developed in this section. In developing the further wind model (2.1), (2.2) and (2.3) are used. In (2.1) coefficients of mechanical power are $\rho\left[\mathrm{kg} / \mathrm{m}^{3}\right]$, and $[\mathrm{m}]$. Similarly the coefficients in (2.2) $C_{1}$ to $C_{10}$ and (2.3) $[\mathrm{m}]$. In this thesis work the data for aerodynamic model is developed by using [72].

Turbine parameters can be drawn by using (2.1). This is possible by calculating the values at the given rated operating condition of the wind turbine. A clear description about calculating the parameters at rated operating condition is given in [66]. From [66] if $P_{\text {rated }}$ is the rated power of the wind turbine, $V_{\text {wrated }}$ is the rated wind speed usually it is in the range of $12-$ $15 \mathrm{~m} / \mathrm{s}$ and the coefficient performance is at its maximum value $C_{p \max }$, then one of the main 
parameter of the wind turbine that is radius can be calculated by using (2.1). This value is defined as $R[m]$ and it is given as [66]:

$$
R=\sqrt{P_{\text {rated }}[W] /\left(0.5 \rho \pi C_{\text {pmax }} V_{\text {wrated }}^{3}\right)}
$$

From (2.5) it can be seen that radius of the wind turbine depends on performance coefficient value $C_{\text {pmax }}$. Finally from the above discussion all the parameters for aerodynamic model can be calculated.

\section{Drive train model:}

Generally in a DFIG based wind generation system the mechanical energy developed by the wind turbine rotor is fed to the generator by means of a gear box. This is because it is not appropriate to drive the generator directly with the mechanical power from the wind turbine rotor or main shaft unless the turbine is equipped with multi-pole generators [71], and moreover the wind turbine rotor cannot produce the desired amount of torque or speed to run the generator. For a better understanding of the necessity of a gearbox in [73], a small example is discussed. Usually a large three-blade wind turbine operates in a speed range of 6-20rpm where as a 4pole, $60 \mathrm{~Hz}$ generator operates at a speed of $1800 \mathrm{rpm}$, so to adapt the low speed of the wind turbine rotor to high speed of the generator a gear box is necessary. Therefore, a gear box usually called as drive train is employed and it acts as an interface between wind turbine rotor and electrical generator to transfer the mechanical energy within the wind generation system.

Usually the drive train is formed by the turbine rotating mass, low speed shaft, the gearbox, high speed shaft and finally the generator rotating mass. With these components a drive train can be represented as a series of rotating disks connected by a shaft. The modeling of drive train is developed by neglecting the mechanical twisting and stresses as these are more related 
to mechanical design and studies. Moreover for power system stability studies it is suggested in [74], to consider the turbine, gearbox and the generator as rigid disks and shafts as mass less torsional springs.

For easy understanding and discussion of drive train model the terminology used for developing the model is presented in this section:

Mass moment of inertia $(H)$ : It is defined as the product of mass $(\mathrm{dm})$ and square of the perpendicular distance $(r)$ between the mass and rotational axis i.e. $H=\int r^{2} d m\left[k g m^{2}\right]$. $H$ describes the mass distribution of a rigid body about an axis.

Shaft stiffness or spring constant $(K)$ : Usually the torsional systems are represented by an equivalent spring mass models. The spring mass model is a type of mechanical link and in most of the applications it is assumed to have negligible mass [75]. If force $F$ is applied on a spring, it undergoes to elongation or reduction in length $x$, then the applied force is give as $F=$ $K x$. In this equation $k$ is a constant and it is known as spring constant or shaft stiffness. It also relates torque to angular displacement defined as [75]:

$$
K=T / \theta[N m / r a d]
$$

In (2.6) $T$ is shaft torque $[\mathrm{Nm}]$ and $\theta[\mathrm{rad}]$ is the twist angle. In other words spring constant or shaft stiffness can be represented in terms of shear modulus or shaft material modulus of rigidity $G\left[N / m^{2}\right]$, moment of inertia $I$ and the shaft length $l[m]$ as [9]:

$$
\begin{aligned}
& K=\frac{G I}{L} \\
& I=\frac{\pi D^{4}}{32}
\end{aligned}
$$

In (2.8) $D$ represents the diameter of the shaft.

Gear Ratio(n): The high speed generator speed is matched with the low speed turbine blades by designing the gear ratio also known as gear box conversion ratio $\left(r_{g b}\right)$. For a given rated 
speed of generator and wind turbine the gear ratio is determined as the ratio of the generator speed to the wind turbine speed in $r p m$ and it is given as [73]: $r_{g b}=\frac{n_{m}}{n_{M}}=\frac{(1-s) \cdot 60 \cdot f_{S}}{P \cdot n_{M}}$. In this equation $n_{m}$ and $n_{M}$ are the generator and turbine rated speed in $r p m, s$ is the rated slip, . $f_{s}$ is the rated stator frequency in $\mathrm{Hz}$, and $P$ is the number of pole pairs of the generator [73]. In [9], the transmission ratio of gear box or gear ratio is represented as the ratio of number of high speed and low speed gears i.e.: $n=\frac{n_{H}}{n_{L}}$. In this equation $n$ is the transmission ratio of gear box, $n_{H}$ and $n_{L}$ are number of high-speed and low-speed gears.

\section{Modeling:}

In this section the mathematical modeling of the drive train is presented. The complete mechanical drive train model used in this thesis work is shown in Figure 2.8.

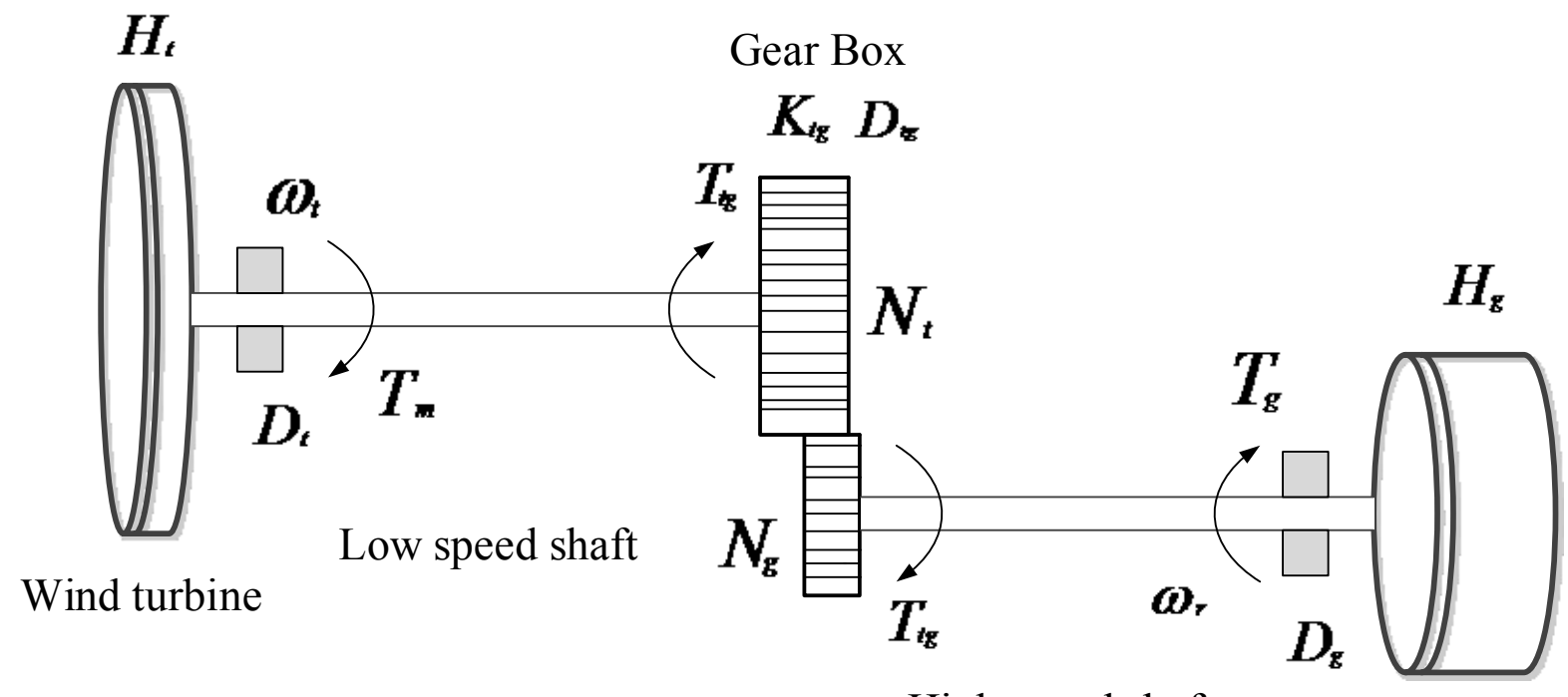

High speed shaft

Figure 2.8: DFIG shaft system, represented by a two-mass model [63]

In Figure $2.8 \omega_{t}$ and $\omega_{r}$ are the turbine and generator rotor speed, respectively. $T_{m}$ and $T_{e}$ are the mechanical torque applied to the turbine and electrical torque respectively; $H_{t}$ and $H_{g}$ are 
the turbine inertia constant and generator inertia constant respectively; $D_{t}$ and $D_{g}$ are the damping coefficients of the turbine and generator respectively; $T_{t g}$ is the internal torque of the model; $D_{t g}$ is the damping coefficient of the shaft between the two masses; $K_{t g}$ is the spring constant or shaft stiffness. Finally $N_{t} / N_{g}$ is the gear ratio of the gear box. It is suggested in [79], the turbine can be represented as a single inertia of the turbine as a lumped-mass model as shown in Figure 2.8 by neglecting the blades and hub with additional rotating masses and shafts. There are three types of drive train models that are used for developing a wind model: a) three mass model, b) two mass model and c) single mass model. The three mass model is considered to be as a direct representation of the entire drive train system with springs and dampers representing the torsion losses on the two shafts. But, the direct implementation of the three mass model is considered as an excessive demand on computational resources during simulating wind turbine generation system [69]. The three mass model is simplified into a two mass model as discussed in [9]. This is done by converting the inertia of the wind turbine, the spring constant, and the damping ratio of the low speed shaft (wind turbine) to the high speed (generator) using [9]:

$$
\begin{aligned}
n & =\frac{n_{H}}{n_{L}} \\
H_{g} & =\frac{H_{t}}{n^{2}} \\
K_{g} & =\frac{K_{t}}{n^{2}} \\
D_{g} & =\frac{D_{t}}{n^{2}}
\end{aligned}
$$

The most commonly used models for power system studies in a wind generation system are the two mass model and the single mass or lumped mass model. Many researches have adopted 
the two mass model [41, 42, 63, 65, 69, 76 and 77]. Similarly lumped mass models are discussed in [9, 76 and 78]. However, in both the models two mass model is recommended for power system stability studies, this is because there is more possibility for the representation of shaft stiffness and inertia constants [80]. Whereas in lumped mass model all the mechanical part of the wind system constituting the wind farm is considered as a single rotating mass [80]. The reason for choosing two mass model over lumped mass model is discussed above. Now the mathematical modeling of the two mass model shown in Figure 2.8 can be obtained as discussed below:

Generally in a wind generation system the wind turbine rotor has larger inertia $\left(H_{t}\right)$ and the inertia of generator $\left(H_{g}\right)$ is less when compared with the wind turbine rotor. The dynamics of the two-mass model can be obtained by applying Newton's equation of motion for each mass. The equations obtained are:

$$
\begin{aligned}
& 2 H_{t} p \omega_{t}=T_{m}-D_{t} \omega_{t}-T_{s h} \\
& 2 H_{g} p \omega_{r}=T_{s h}-D_{g} \omega_{r}-T_{e}
\end{aligned}
$$

Where $p=d / d t$ and $T_{s h}$ is the shaft torque and it is given as:

$$
T_{s h}=D_{t g}\left(\omega_{t}-\omega_{r}\right)+T_{t g}
$$

By substituting (2.16) in (2.13) - (2.15), the electromechanical dynamic equations are obtained:

$$
\begin{gathered}
2 H_{t} p \omega_{t}=T_{m}-D_{t} \omega_{t}-D_{t g}\left(\omega_{t}-\omega_{r}\right)-T_{t g} \\
2 H_{g} p \omega_{r}=D_{t g}\left(\omega_{t}-\omega_{r}\right)+T_{t g}-D_{g} \omega_{r}-T_{e} \\
p T_{t g}=K_{t g}\left(\omega_{t}-\omega_{r}\right)
\end{gathered}
$$

The variables used in equations $(2.13)-(2.18)$ are discussed above. In (2.16) $T_{m}$ is the mechanical torque obtained from wind turbine and it is derived below. In (2.17) $T_{e}$ is the electrical torque of the generator and it is given as $T_{e}=\psi_{q r} i_{q r}-\psi_{d r} i_{q r}$. A clear description 
about electrical torque is obtained from modeling the generator in Section 2.4.2. The above derived modeling is obtained from [63 and 76].

From (2.1) the mechanical torque $T_{m}$ is given as:

$$
T_{m}=\frac{P_{t}}{\omega_{t}}
$$

\section{Lumped mass or one-mass model:}

The lumped mass model is formed by combining the turbine, gearbox and the shafts together into an equivalent mass inertia $H_{m}$, the electromechanical dynamic equation obtained is as follows [63]:

$$
2 H_{m} p \omega_{m}=T_{m}-D_{m} \omega_{m}-T_{e}
$$

In (2.16), $\omega_{m}$ is the rotational speed of the lumped mass system and $\omega_{m}=\omega_{t}=\omega_{r}$; and $D_{m}$ is the damping of the lumped mass model.

\subsubsection{Electrical generator (DFIG) model:}

Doubly fed induction generators are the most commonly used and widely accepted generators for the large wind power generation. As mentioned in Subsection 2.2.3 (c) the constructional features of DFIG are similar to that of the wound rotor and squirrel cage induction generators, except that the rotor windings are not shorted, therefore the voltage of rotor windings is not equal to zero. A typical block diagram of connecting a DFIG in a wind generation system can be observed from Figure 2.6. The main advantage of DFIG based wind generation system is that, the usage of a back to back power electronic converter which handles only a fraction of total output power, thus leading to reduction of losses in power electronic converters. This fraction of output power depends on the allowable sub and super synchronous speed range [81]. Where has in fully converter based systems the losses caused by power electronic devices is more as the total output power is transmitted through this equipment. The other advantages 
for usage of DFIG for wind generation applications include from the characteristics of doubly fed induction generator they are [81]: 1) A DFIG can supply power at constant voltage and frequency while the rotor speed varies, this makes DFIG more suitable for wind generation applications. 2) The speed range can be extended above and below synchronous speeds along with generation of power from both stator and rotor. 3) Supply of low distortion currents to the main supply and control over system power factor [27].

A doubly fed induction generator consists of two three phase winding sets, each contributing to stator and rotor respectively, and both the stator and rotor windings are capable of supplying energy bidirectional [54]. Depending on the operating condition the power can be delivered from the rotor winding to the grid or it can be supplied to the rotor winding from the grid through back to back converter.

The DFIG with $p$ pairs of poles, when supplied by a balance three phase voltage of frequency $f_{s}$, the stator flux is induce in the stator. With this the stator flux starts rotating at a constant speed, this is synchronous speed $n_{s}$, and it is expressed as [54]:

$$
n_{s}=\frac{60 f_{s}}{p}(r p m)
$$

From Faraday's law of induction this stator flux induces an electro motive force (emf) in the rotor windings, due to which a current is induced in the rotor winding. According to Laplace's law this induced current creates an induced force in the rotor of the DFIG machine. From this discussion it can be observed that the induced voltage depends on the rotational speed of stator flux and rotational speed of the rotor. The relation between angular frequency of the induced rotor voltages and currents is given by [54]: $\omega_{r}=\omega_{s}-\omega_{m}$; in this equation $\omega_{r}$ is the angular frequency of the voltages and currents of the rotor windings $(\mathrm{rad} / \mathrm{s}) ; \omega_{s}$ is the angular frequency of the voltages and currents of the stator windings $(\mathrm{rad} / \mathrm{s})$; and $\omega_{m}$ is the angular 
frequency of the rotor $(\mathrm{rad} / \mathrm{s})$. Finally from this the slip $(s)$ is obtained which shows relation between the speed of stator and the rotor angular frequency. It is expressed as [54]:

$$
s=\frac{\omega_{s}-\omega_{m}}{\omega_{s}}
$$

From (2.18), relation between rotor angular frequency, the lip and stator angular frequency as well as between the stator and rotor frequencies can be obtained and it is given as:

$$
\begin{gathered}
\omega_{r}=s \omega_{s} \\
f_{r}=s f_{s}
\end{gathered}
$$

Based on the rotor speed derived in (2.19); operation of DFIG can be classified into two modes:

a) Super-synchronous mode of operation

b) Sub-synchronous mode of operation.

a) Super-synchronous mode of operation [32]: Induction generator operates in super synchronous mode of operation when the slip is negative, and this is possible only when the speed of the generator is above the synchronous speed $\left(\omega_{s}\right)$. This can be explained clearly by using (2.18) from which it can be observed that, during the super-synchronous mode of operation $\left(\omega_{m}>\omega_{s}\right)$ thus leading to $\omega_{r}<0$ which finally indicates that slip $s<0$. In this mode of operation the mechanical power $\left(P_{t}\right)$, from the wind turbine, is delivered to the grid from both the stator winding and rotor winding of the generator. As shown in Figure 2.9 (a), stator power $\left(P_{S}\right)$ is directly fed to the grid whereas rotor power $\left(P_{r}\right)$ is fed through power electronic converter circuit. The power distribution in super-synchronous mode of operation can be observed in Figure 2.9 (a) [32].

b) Sub-synchronous mode of operation [32]: In this mode of operation the DFIG operates below the synchronous speed $\left(\omega_{s}\right)$, thus slip is positive in this mode of operation. This can be explained clearly by using (2.18), in sub-synchronous mode of operation $\left(\omega_{m}<\omega_{s}\right)$ thus 
leading to $\omega_{r}>0$ which finally indicates that $\operatorname{slip} s>0$. In this mode of operation the mechanical power $\left(P_{t}\right)$, from the wind turbine is delivered to the grid from the stator winding of the generator only. Whereas the rotor of the generator draws power from the grid through the converter circuit. This power is fed along with the mechanical power $\left(P_{t}\right)$ to the generator stator. So the total power delivered to the grid is only through the stator of the generator and it is the total of mechanical power and the rotor power $\left(P_{t}+P_{r}\right)$. The important thing is that in sub-synchronous mode of operation even though the total power is fed by the stator, it will not exceed the power rating of the machine as the mechanical power from the generator shaft is lower than the super-synchronous mode [32]. The distribution of power in sub-synchronous model of operation can be observed in Figure 2.9 (b) [32].

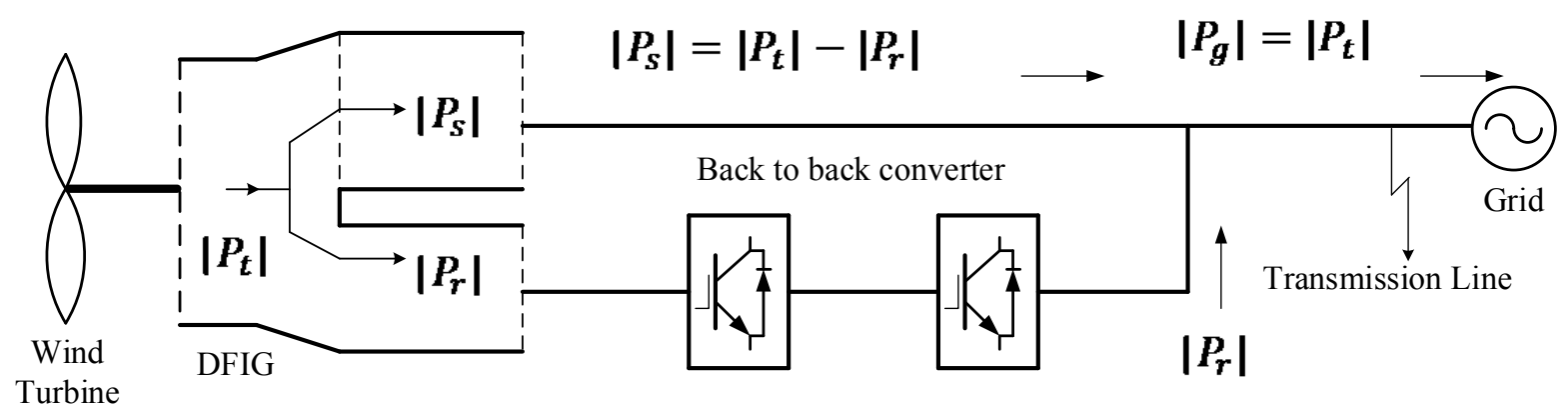

Figure 2.9 (a): Super synchronous mode

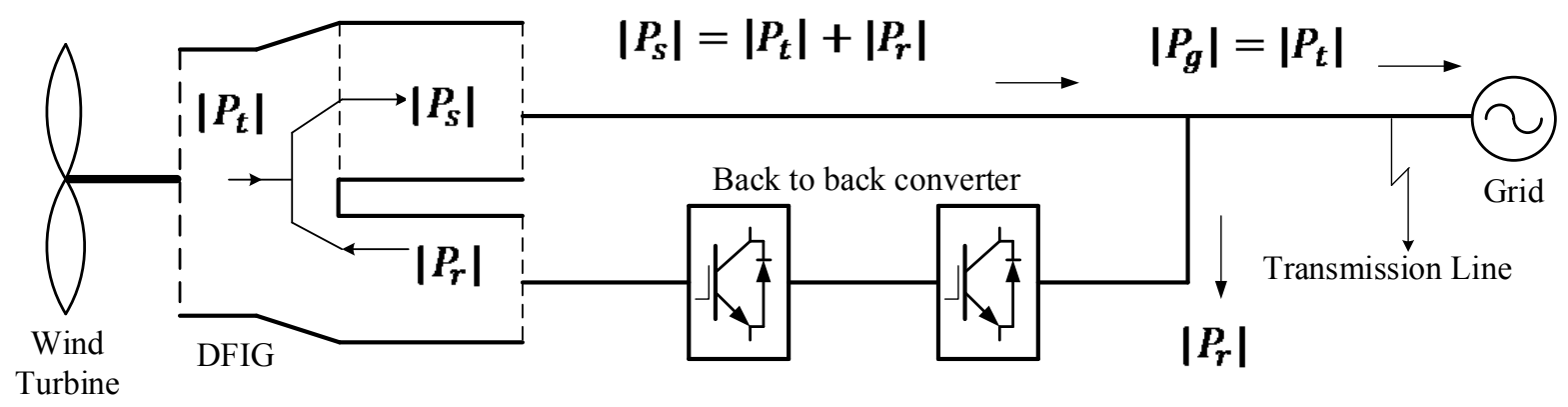

Figure 2.9(b): Sub- synchronous mode

However the doubly fed induction generator can be operated as both generator and as well as motor in both super-synchronous and sub-synchronous model of operation using the 
controllers. But in this thesis the research interest is on generating mode of operation. This can be made clear by observing the torque speed characteristics as shown in Figure 2.10. From the figure it can be observed that the DFIG machine can be operated in both generator and motor mode with respect to the rotor speed $\omega_{r}$ along the synchronous speed $\omega_{s}$.

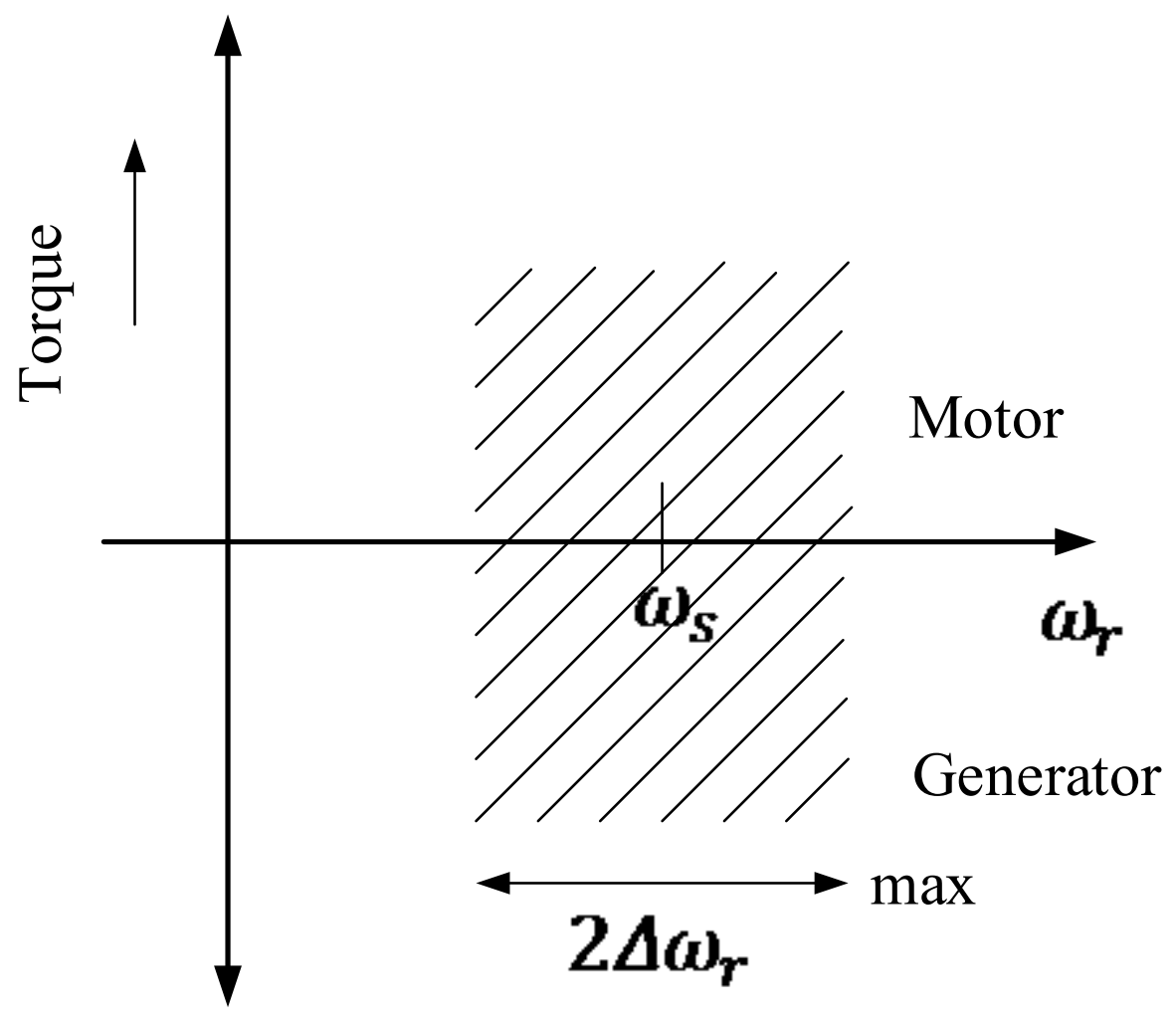

Figure 2.10: Speed- torque characteristics of DFIG

In this thesis the DFIG machine based on which the analysis is performed, is operated as a generator.

For power system stability studies the DFIG machine is modeled by neglecting stator transients as they do not affect the electromechanical oscillations [82] and by neglecting stator transients it is easier to solve for stator and grid equations [83]. Similarly the rotor electrical transients are also neglected as the rotor winding is controlled by the fast acting converters [65]. In addition, the other assumptions that are made for modeling the DFIG are: the skin effect, the 
saturation effect and the iron losses (hysteresis and eddy currents) are neglected as these phenomena contribute more for conducting loss performance, transient fault analysis.

\section{DFIG steady state model:}

Prior to discussing the dynamic model of DFIG, for an easy and clear understanding DFIG steady state model is presented in this subsection. It is assumed that both the stator and rotor are connected in star winding and stator is supplied by a constant voltage by the grid. The analysis is carried out for a 1-phase system as shown in Figure 2.11.

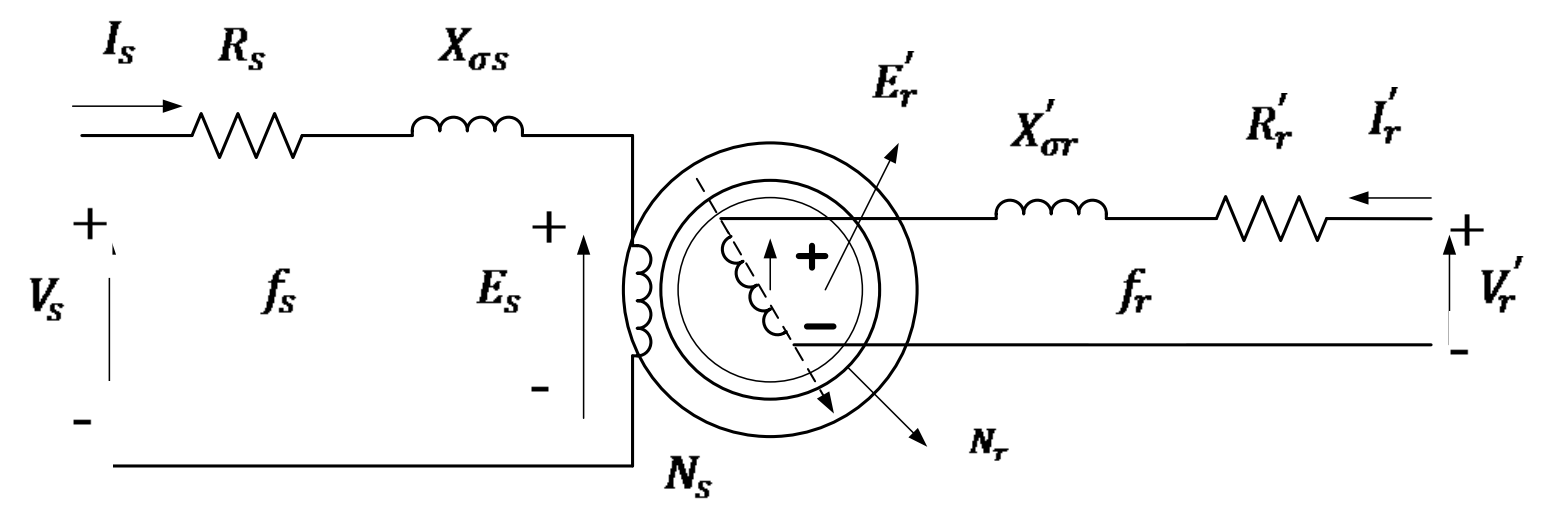

Figure 2.11:Single-phase steady state equivalent electric circuit of DFIG [54]

The steady state model equations for the circuit shown above are derived as follows [54]:

Stator model:

$$
V_{s}=R_{s} I_{s}+j \omega_{s} L_{\sigma s}+E_{s}
$$

Where $V_{S}$ is supplied stator voltage at frequency $f_{s} ; E_{s}$ is induced emf in stator winding; $I_{s}$ is induced stator current; $R_{S}$ is stator resistance; $X_{\sigma s}=j \omega_{S} L_{\sigma s}$ is stator leakage impedance.

Rotor model:

$$
V_{r}^{\prime}=R_{r}^{\prime} I_{r}^{\prime}+j \omega_{r} L_{\sigma r}^{\prime}+E_{r}^{\prime}(2.22)
$$

$V_{r}^{\prime}$ is the supplied rotor voltage at frequency $f_{r} ; E_{r}^{\prime}$ is induced emf in rotor winding; $I_{r}^{\prime}$ is induced rotor current; $R_{r}^{\prime}$ is rotor resistance; $X_{\sigma r}^{\prime}=j \omega_{s} L_{\sigma r}^{\prime}$ is rotor leakage impedance. In 
Figure 2.11, $N_{s}$ is number of stator winding turns per phase whereas $N_{r}$ is number of rotor winding turns per phase. In (2.21) and (2.22) voltages and currents are represented as rms values.

Induced emf in stator and rotor are given as:

$$
\begin{aligned}
& E_{s}=\sqrt{2} \pi N_{s} f_{s} K_{s} \Phi_{m} \\
& E_{r}^{\prime}=\sqrt{2} \pi N_{r} f_{r} K_{r} \Phi_{m}
\end{aligned}
$$

$K_{s}$ and $K_{r}$ are winding factors for stator and rotor respectively. Referring rotor variables to stator side the obtained variables are: $R_{r}=R_{r}^{\prime}\left(\frac{N_{s}}{N_{r}}\right)^{2} ; L_{\sigma r}=L_{\sigma r}^{\prime}\left(\frac{N_{S}}{N_{r}}\right)^{2} ; V_{r}=V_{r}^{\prime}\left(\frac{N_{s}}{N_{r}}\right) ; I_{r}=$ $I_{r}^{\prime}\left(\frac{N_{r}}{N_{S}}\right) ; E_{r}=E_{r}^{\prime}\left(\frac{N_{S}}{N_{r}}\right)$. From this the rotor final equation referred to stator can be defined as:

$$
V_{r}=R_{r} I_{r}+j \omega_{r} L_{\sigma r}+E_{r}
$$

Equation (2.25) represents rotor equation at frequency $f_{r}$. From (2.21) and (2.25) an equivalent circuit can be defined as shown in Figure 2.12. In this case the frequencies are different for stator and rotor.

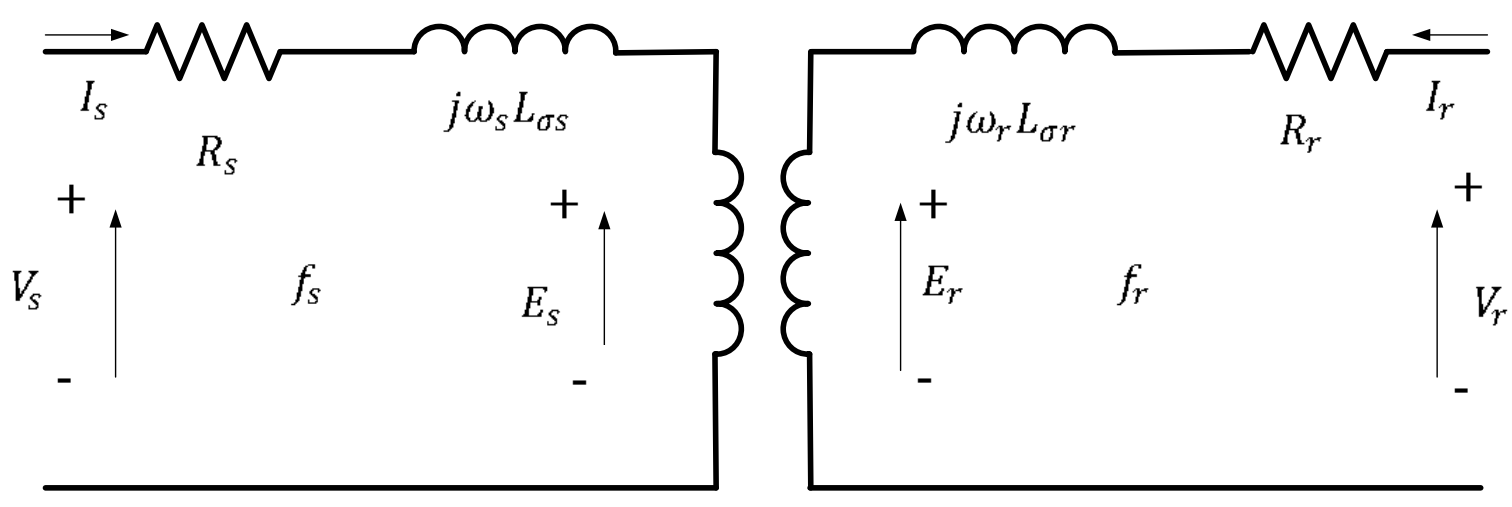

Figure 2.12: DFIG steady state equivalent circuit with different frequencies [54] An equation can be obtained by transforming rotor frequency to stator frequency by dividing (2.25) with the slip $s$. The obtained model is: 


$$
\frac{V_{r}}{S}=\frac{R_{r}}{S}+j \omega_{s} L_{\sigma r}^{\prime}+\frac{E_{r}}{S}
$$

Final steady state equivalent circuit is shown in Figure 2.13, obtained from 2.26.

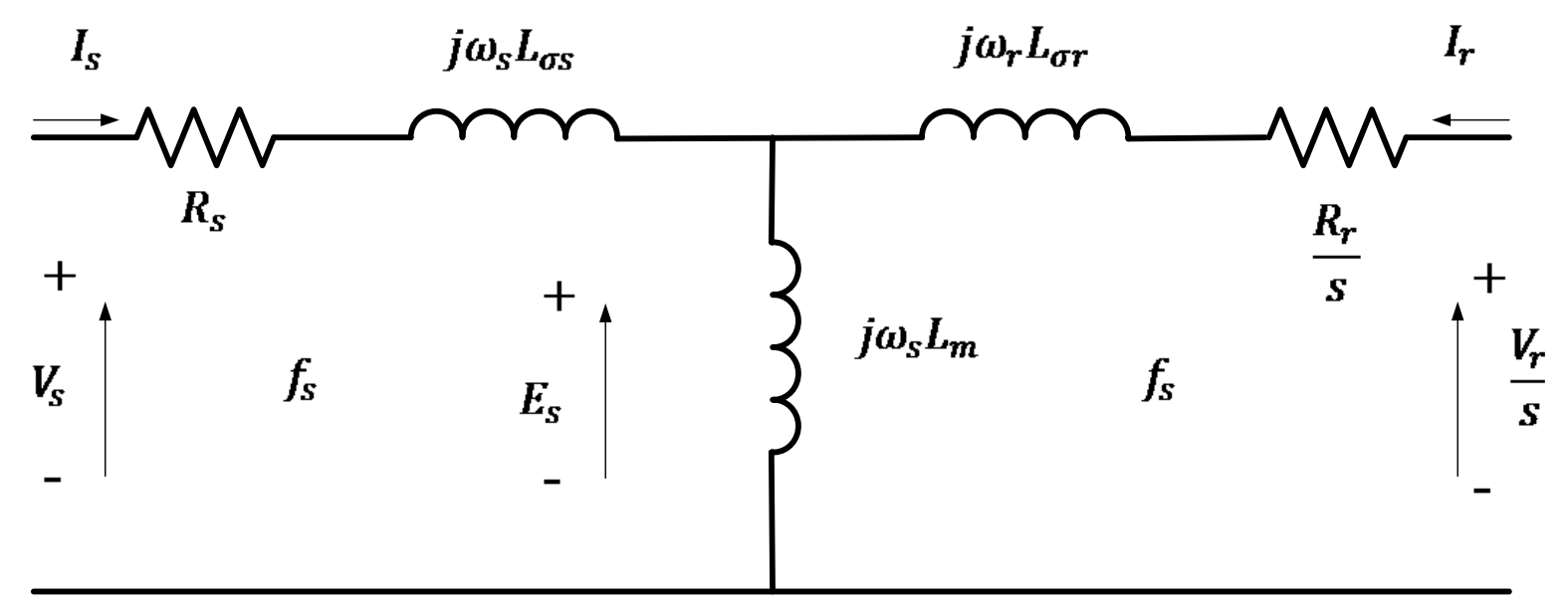

Figure 2.13: DFIG steady state equivalent circuit referred to stator

From the steady state equivalent model shown in Figure 2.13, active power can be derived, similarly stator and rotor flux equations can be expressed in terms of currents and inductances as shown below:

$$
\psi_{m}=L_{m}\left(I_{s}+I_{r}\right)
$$

Stator flux equation is expressed as $\psi_{s}=L_{s} I_{s}+L_{m} I_{r}=\left(L_{\sigma s}+L_{m}\right) I_{s}+L_{m} I_{r}$

$$
\psi_{s}=L_{\sigma s} I_{s}+\psi_{m}
$$

Similarly rotor flux is given as $\psi_{r}=L_{r} I_{r}+L_{m} I_{s}=\left(L_{\sigma r}+L_{m}\right) I_{r}+L_{m} I_{s}$

$$
\psi_{r}=L_{\sigma r} I_{r}+\psi_{m}
$$

$\psi_{m}, \psi_{s}$ and $\psi_{r}$ denote the mutual flux, stator flux and rotor flux respectively. Where as $L_{s}$ and $L_{r}$ denote stator and rotor inductance respectively.

From this the new voltage equations can be represented as:

$$
\begin{aligned}
& V_{s}=R_{s} I_{s}+j \omega_{s} \psi_{s} \\
& V_{r}=R_{r} I_{r}+j \omega_{r} \psi_{r}
\end{aligned}
$$




\section{Dynamic modeling of DFIG:}

The steady state model presented previously is further developed in to a dynamic model. Representing the machine by its dynamic model is important as it is required for designing and developing of the machine controllers. By using the dynamic model of the machine it is possible to understand the performance of different parameters related to machine.

\section{Model in abc-frame:}

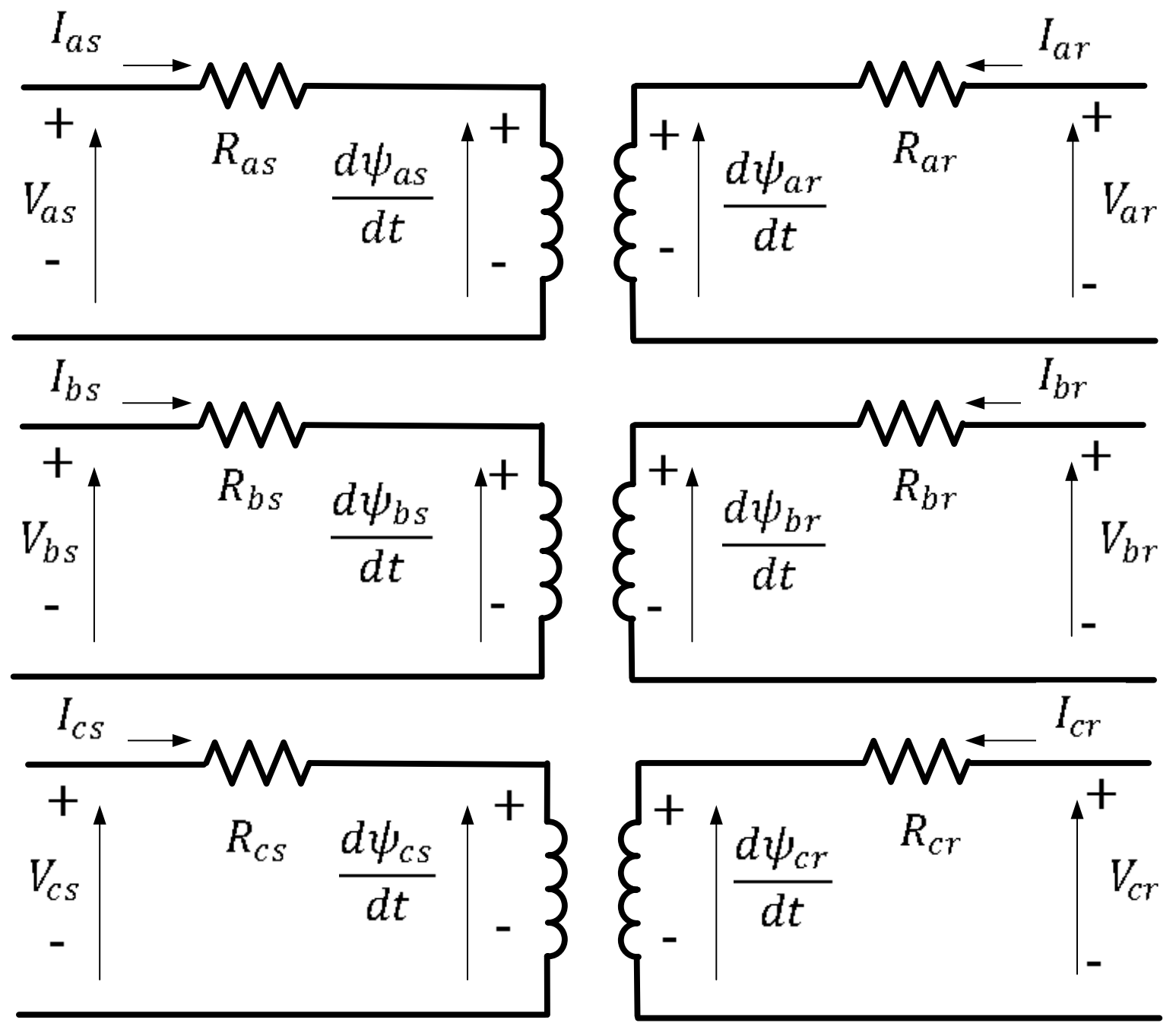

Figure 2.14: Electrical equivalent circuit of DFIG

The three phase differential equations of doubly fed induction machine can be obtained by applying Kirchhoff's voltage law to both the stator and rotor circuits of the machine. The 
differential equations can be described as follows by using the electrical equivalent model of the machine represented in $a b c$ frame as shown in Figure 2.14:

Stator equations:

$$
\begin{aligned}
& v_{a s}=R_{s} i_{a s}+\frac{d \psi_{a s}}{d t} \\
& v_{b s}=R_{s} i_{b s}+\frac{d \psi_{b s}}{d t} \\
& v_{c s}=R_{s} i_{c s}+\frac{d \psi_{c s}}{d t}
\end{aligned}
$$

Where, $v_{a s}, v_{b s}$ and $v_{c s}$ are the applied stator voltages; $i_{a s}, i_{b s}$ and $i_{c s}$ are stator currents and $\psi_{a s}, \psi_{b s}$ and $\psi_{c s}$ are the stator fluxes.

Rotor side equations can be defined as:

$$
\begin{aligned}
& v_{a r}=R_{r} i_{a r}+\frac{d \psi_{a r}}{d t} \\
& v_{b r}=R_{r} i_{b r}+\frac{d \psi_{b r}}{d t} \\
& v_{c r}=R_{r} i_{c r}+\frac{d \psi_{c r}}{d t}
\end{aligned}
$$

Where, $v_{a r}, v_{b r}$ and $v_{c r}$ are the rotor voltages referred to stator; $i_{a r}, i_{b r}$ and $i_{c r}$ are stator referred rotor currents and finally $\psi_{a r}, \psi_{b r}$ and $\psi_{c r}$ are the rotor fluxes. Voltage equations for stator and rotor can be expressed as:

$$
\begin{aligned}
& \left(f_{a b c s}\right)^{T}=\left[\begin{array}{lll}
f_{a s} & f_{b s} & f_{c s}
\end{array}\right] \\
& \left(f_{a b c r}\right)^{T}=\left[\begin{array}{lll}
f_{a r} & f_{b r} & f_{c r}
\end{array}\right]
\end{aligned}
$$

For a magnetically linear system the flux linkages can be expressed as [84]:

$$
\left[\begin{array}{l}
\psi_{a s} \\
\psi_{b s} \\
\psi_{c s}
\end{array}\right]=\left[\begin{array}{ccc}
L_{a s} & L_{a b s} & L_{a c s} \\
L_{b a s} & L_{b s} & L_{b c s} \\
L_{c a s} & L_{c b s} & L_{c s}
\end{array}\right]\left[\begin{array}{l}
i_{a s} \\
i_{b s} \\
i_{c s}
\end{array}\right]+\left[\begin{array}{lll}
L_{a s a r} & L_{a s b r} & L_{a s c r} \\
L_{b s a r} & L_{b s b r} & L_{b s c r} \\
L_{c s a r} & L_{c s b r} & L_{c s c r}
\end{array}\right]\left[\begin{array}{l}
i_{a r} \\
i_{b r} \\
i_{c r}
\end{array}\right]
$$


In (2.40), $L_{a s}, L_{b s}$ and $L_{c s}$ are stator winding self-inductances; $L_{a b s}, L_{a c s}, L_{b a s}, L_{b c s}, L_{c a s}$ and $L_{c b s}$ are mutual inductances between stator windings. $\left[\begin{array}{lll}L_{a s a r} & L_{a s b r} & L_{a s c r} \\ L_{b s a r} & L_{b s b r} & L_{b s c r} \\ L_{c s a r} & L_{c s b r} & L_{c s c r}\end{array}\right]=L_{s r}$; represent mutual inductances between stator and rotor winding.

Similarly rotor fluxes can be represented as:

$$
\left[\begin{array}{l}
\psi_{a r} \\
\psi_{b r} \\
\psi_{c r}
\end{array}\right]=\left[\begin{array}{ccc}
L_{a r} & L_{a b r} & L_{a c r} \\
L_{b a r} & L_{b r} & L_{b c r} \\
L_{c a r} & L_{c b r} & L_{c r}
\end{array}\right]\left[\begin{array}{l}
i_{a r} \\
i_{b r} \\
i_{c r}
\end{array}\right]+\left[\begin{array}{lll}
L_{a r a s} & L_{a r b s} & L_{a r c s} \\
L_{b r a s} & L_{b r b s} & L_{b r c s} \\
L_{c r a s} & L_{c r b s} & L_{c r c s}
\end{array}\right]\left[\begin{array}{l}
i_{a s} \\
i_{b s} \\
i_{c s}
\end{array}\right]
$$

In (2.41), $L_{a r}, L_{b r}$ and $L_{c r}$ are rotor winding self-inductances; $L_{a b r}, L_{a c r}, L_{b a r}, L_{b c r}, L_{c a r}$ and $L_{c b r}$ are mutual inductances between rotor windings. $\left[\begin{array}{lll}L_{\text {aras }} & L_{\text {arbs }} & L_{\text {arcs }} \\ L_{\text {bras }} & L_{b r b s} & L_{b r c s} \\ L_{c r a s} & L_{c r b s} & L_{c r c s}\end{array}\right]=L_{r s}=$ $\left(L_{s r}\right)^{T}$; represent mutual inductances between stator and rotor winding.

Where:

$$
\begin{gathered}
L_{s s}=\left[\begin{array}{ccc}
L_{a s} & L_{a b s} & L_{a c s} \\
L_{b a s} & L_{b s} & L_{b c s} \\
L_{c a s} & L_{c b s} & L_{c s}
\end{array}\right]=\left[\begin{array}{ccc}
L_{l s}+L_{m s} & -\frac{1}{2} L_{m s} & -\frac{1}{2} L_{m s} \\
-\frac{1}{2} L_{m s} & L_{l s}+L_{m s} & -\frac{1}{2} L_{m s} \\
-\frac{1}{2} L_{m s} & -\frac{1}{2} L_{m s} & L_{l s}+L_{m s}
\end{array}\right] \\
L_{r r}=\left[\begin{array}{cccc}
L_{a r} & L_{a b r} & L_{a c r} \\
L_{b a r} & L_{b r} & L_{b c r} \\
L_{c a r} & L_{c b r} & L_{c r}
\end{array}\right]=\left[\begin{array}{ccc}
L_{l r}+L_{m r} & -\frac{1}{2} L_{m r} & -\frac{1}{2} L_{m r} \\
-\frac{1}{2} L_{m r} & L_{l r}+L_{m r} & -\frac{1}{2} L_{m r} \\
-\frac{1}{2} L_{m r} & -\frac{1}{2} L_{m r} & L_{l r}+L_{m r}
\end{array}\right] \\
L_{s r}=\left(L_{m s r}\right)\left[\begin{array}{ccc}
\cos \theta_{r} & \cos \left(\theta_{r}+\frac{2 \pi}{3}\right) & \cos \left(\theta_{r}-\frac{2 \pi}{3}\right) \\
\cos \left(\theta_{r}-\frac{2 \pi}{3}\right) & \cos \theta_{r} & \cos \left(\theta_{r}+\frac{2 \pi}{3}\right) \\
\cos \left(\theta_{r}+\frac{2 \pi}{3}\right) & \cos \left(\theta_{r}-\frac{2 \pi}{3}\right) & \cos \theta_{r}
\end{array}\right]
\end{gathered}
$$

From these equations flux equations in $a b c$ reference frame can be represented as: 


$$
\begin{aligned}
& \psi_{a b c s}=L_{s s} i_{a b c s}+L_{s r} i_{a b c r} \\
& \psi_{a b c r}=L_{r r} i_{a b c r}+L_{r s} i_{a b c s}
\end{aligned}
$$

In (2.42) - (2.46) $L_{l s}$ and $L_{m s}$ are leakage and magnetizing inductances if the stator winding; $L_{l r}$ and $L_{m r}$ are for the rotor windings and $L_{m s r}$ is the amplitude of the mutual inductance between stator and rotor windings. The derivation for the above matrices can be found in [84]. These voltage equations describe the performance of the DFIG machine in $a b c$ reference frame. In practice the above derived generator model is represented in rotating two axis reference frame. The system is represented in two axis reference frame for an easy control over the variables and to reduce the complexity of the differential equations which describe the behavior of the machine [84]. There are three different reference frames used to represent the machine in two axis reference frame: a) Stator reference frame; b) Rotor reference frame and c) Synchronous reference frame. However, in most of the cases the induction machine is represented either in synchronously rotating reference frame or stationary reference frame [85]. In stationary reference frame the rotor variables are transformed to a frame of reference fixed in stator [84], i.e. the rotating speed of the frame is zero (stationary). In rotor reference frame theory the stator variables are referred to the rotor and the rotating speed of the frame is the angular speed of the rotor. Finally in synchronous reference frame both the stator and rotor variables are referred to a reference frame rotating in synchronism with the rotating field. The rotating speed of the frame is its synchronous peed [84].

\section{Model in synchronous (qd0) reference frame:}

It can be observed from the flux equations (2.45) and (2.46), the variables $L_{s s}$ and $L_{s r}$ are constants and the variables related to $L_{s r}$ and $L_{r s}$ are time dependent, this can be observed from (2.44) which changes with the change in angle. So, the system in $a b c$ reference frame is 
time varying. This can be made time invariant by converting the system to a two-axis reference frame in which the variables are shifted to a common rotating reference frame.

Transformation from $a b c$ reference frame to $q d 0$ reference frame is carried out by using Parks transformation method. The transformation equation is $[84,86]$ :

$$
f_{q d 0 s}=T_{s} f_{a b c}
$$

$\left(f_{\text {abcs }}\right)^{T}$ is same as $(2.38)$ where as $\left(f_{q d 0}\right)^{T}=\left[\begin{array}{lll}f_{q s} & f_{d s} & f_{0 s}\end{array}\right]$

$$
T_{S}=\frac{2}{3}\left[\begin{array}{ccc}
\cos \theta & \cos \left(\theta-\frac{2 \pi}{3}\right) & \left.\cos \left(\theta+\frac{2 \pi}{3}\right)\right) \\
\sin \theta & \sin \left(\theta-\frac{2 \pi}{3}\right) & \sin \left(\theta+\frac{2 \pi}{3}\right) \\
\frac{1}{2} & \frac{1}{2} & \frac{1}{2}
\end{array}\right]
$$

Where $\theta$ is the transformation angle between the $q$ axis and $a$ axis as shown in Figure 2.15:

$$
\theta=\int_{0}^{t} \omega+\theta(0)
$$

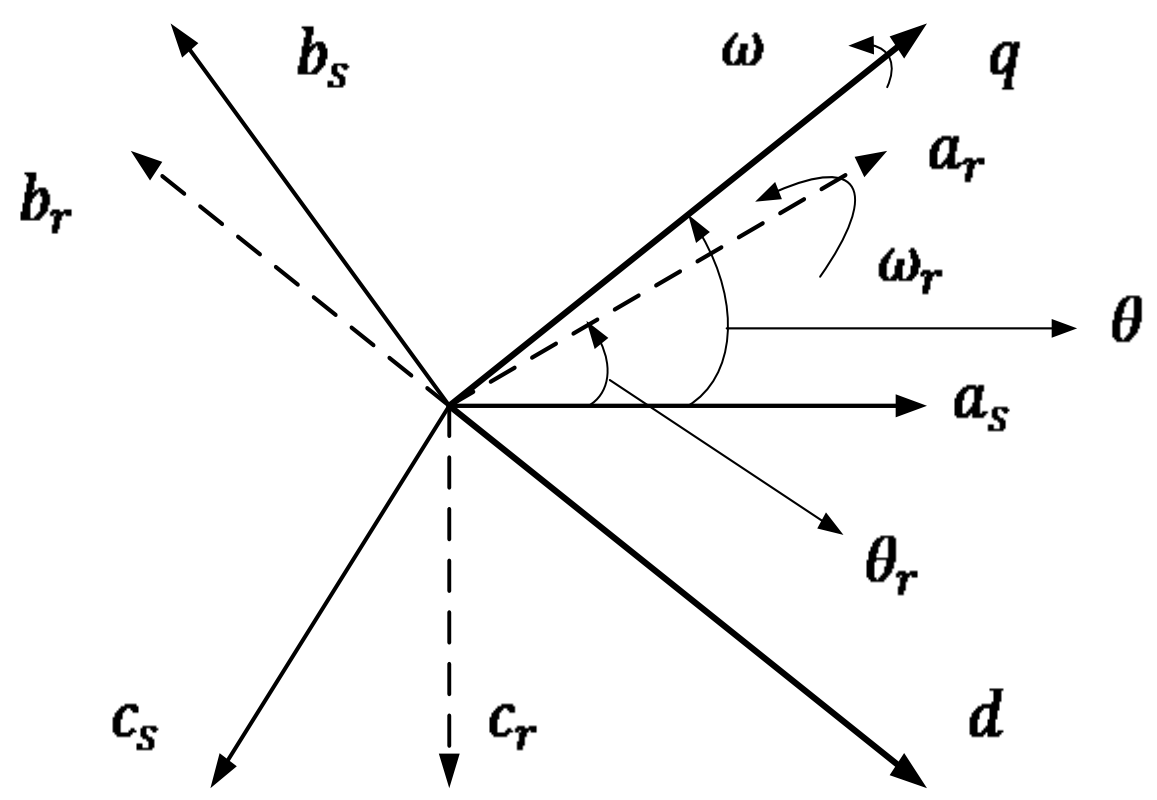

Figure 2.15: two axis reference frame for DFIG

In Figure $2.15 q$ and $d$ axis are orthogonal to each other rotating at an angular velocity of $\omega$. 
Whereas $a_{s}, b_{s}$ and $c_{s}$ represent stator variables displaced by $120^{\circ}$. Matrix represented in (2.47) is used for transforming stator variables. For analyzing an induction machine variables associated with the rotor also need to be transformed to $q d$ axis reference frame. From Figure $2.15 a_{r}, b_{r}$ and $c_{r}$ represent rotor variables displaced by $120^{\circ}$ rotating at an angular velocity of $\omega_{r}$. The angular position between the $q d$ axis reference frame and rotor $a b c$ reference frame is $\left(\theta-\theta_{r}\right)$.

$$
\theta_{r}=\int_{0}^{t} \omega_{r}+\theta_{r}(0)
$$

The transformation equation for rotor variables is $[84,86]$ :

$$
f_{q d r 0 s}=T_{r} f_{a b c r}
$$

$\left(f_{a b c r}\right)^{T}$ is same as (2.39) where as $\left(f_{q d r 0}\right)^{T}=\left[\begin{array}{lll}f_{q r} & f_{d r} & f_{0 r}\end{array}\right]$

$$
T_{r}=\frac{2}{3}\left[\begin{array}{ccc}
\cos \left(\theta-\theta_{r}\right) & \cos \left(\theta-\theta_{r}-\frac{2 \pi}{3}\right) & \cos \left(\theta-\theta_{r}+\frac{2 \pi}{3}\right) \\
\sin \left(\theta-\theta_{r}\right) & \sin \left(\theta-\theta_{r}-\frac{2 \pi}{3}\right) & \sin \left(\theta-\theta_{r}+\frac{2 \pi}{3}\right) \\
\frac{1}{2} & \frac{1}{2} & \frac{1}{2}
\end{array}\right]
$$

By using (2.47) and (2.51) and there transpose matrices, transformed equations can be obtained by implementing the transformation procedure given in $[84,86]$. As it is discussed the main two reference frames which are used to describe an induction machine are the stationary reference frame and synchronous reference frame. However in this thesis synchronous reference frame is used. This is because for power system studies to conduct steady-state analysis and derive small signal model the convenient way of using $q d$ axis model is by representing the model in synchronously rotating reference frame [86]. In synchronous reference frame the $q d$ axis rotate at an angular speed $\omega_{s}$. So, the angular speed represented in Figure 2.15, is represented as $\omega=\omega_{s}$ in synchronously rotating reference frame. By 
transforming the model in to synchronously rotating $q d$ axis reference frame the obtained transformed equations for DFIG in per unit are:

Stator model represented in $q d$ axis reference frame

$$
\begin{aligned}
& v_{d s}=r_{s} i_{d s}-\frac{\omega_{s}}{\omega_{b}} \psi_{q s}+p \psi_{d s} \\
& v_{q s}=r_{s} i_{q s}+\frac{\omega_{s}}{\omega_{b}} \psi_{d s}+p \psi_{q s}
\end{aligned}
$$

Rotor model represented in $q d$ axis reference frame

$$
\begin{aligned}
& v_{d r}=r_{r} i_{d r}-\left(\frac{\omega_{s}-\omega_{r}}{\omega_{b}}\right) \psi_{q r}+p \psi_{d r} \\
& v_{q r}=r_{r} i_{q r}+\left(\frac{\omega_{s}-\omega_{r}}{\omega_{b}}\right) \psi_{d r}+p \psi_{q r}
\end{aligned}
$$

Flux linkages are given as:

$$
\begin{gathered}
\psi_{d s}=L_{s} i_{d s}+L_{m} i_{d r} \\
\psi_{q s}=L_{s} i_{q s}+L_{m} i_{q r} \\
\psi_{d r}=L_{r} i_{d r}+L_{m} i_{d s} \\
\psi_{q r}=L_{r} i_{q r}+L_{m} i_{q s} \\
L_{s}=L_{l s}+L_{m} \\
L_{r}=L_{l r}+L_{m}
\end{gathered}
$$

In this model $p=\frac{d}{d t} ; \omega_{s}$ is the rotational speed of the synchronous reference frame, $\omega_{r}$ is the rotor speed of the machine, $L_{l s}$ is the stator leakage $L_{l r}$ is the rotor leakage inductance referred to stator and $L_{m}$ is the mutual inductance. $r_{s}$ is the stator resistance while $r_{r}$ is the resistance of the rotor referred to stator. $\omega_{b}$ is the base electrical angular velocity.

The electromagnetic torque equation and the active and reactive power equations can be obtained from the above equations in terms of $q d$ components. By neglecting the power losses 
in stator and rotor the active and reactive powers for stator and rotor of the machine in $q d$ reference frame can be expressed as:

$$
\begin{gathered}
P_{s}=\frac{3}{2}\left(v_{d s} i_{d s}+v_{q s} i_{q s}\right) \\
Q_{s}=\frac{3}{2}\left(v_{q s} i_{d s}-v_{d s} i_{q s}\right) \\
P_{r}=\frac{3}{2}\left(v_{d r} i_{d r}+v_{q r} i_{q r}\right) \\
Q_{r}=\frac{3}{2}\left(v_{q r} i_{d r}-v_{d r} i_{q r}\right)
\end{gathered}
$$

The per unit electromagnetic torque equation in $q d$ components can be expressed as:

$$
T_{e}=L_{m}\left(i_{q s} i_{d r}-i_{d s} i_{q r}\right)
$$

The equivalent circuit of a DFIG in synchronously rotating reference is given in Figure 2.16.

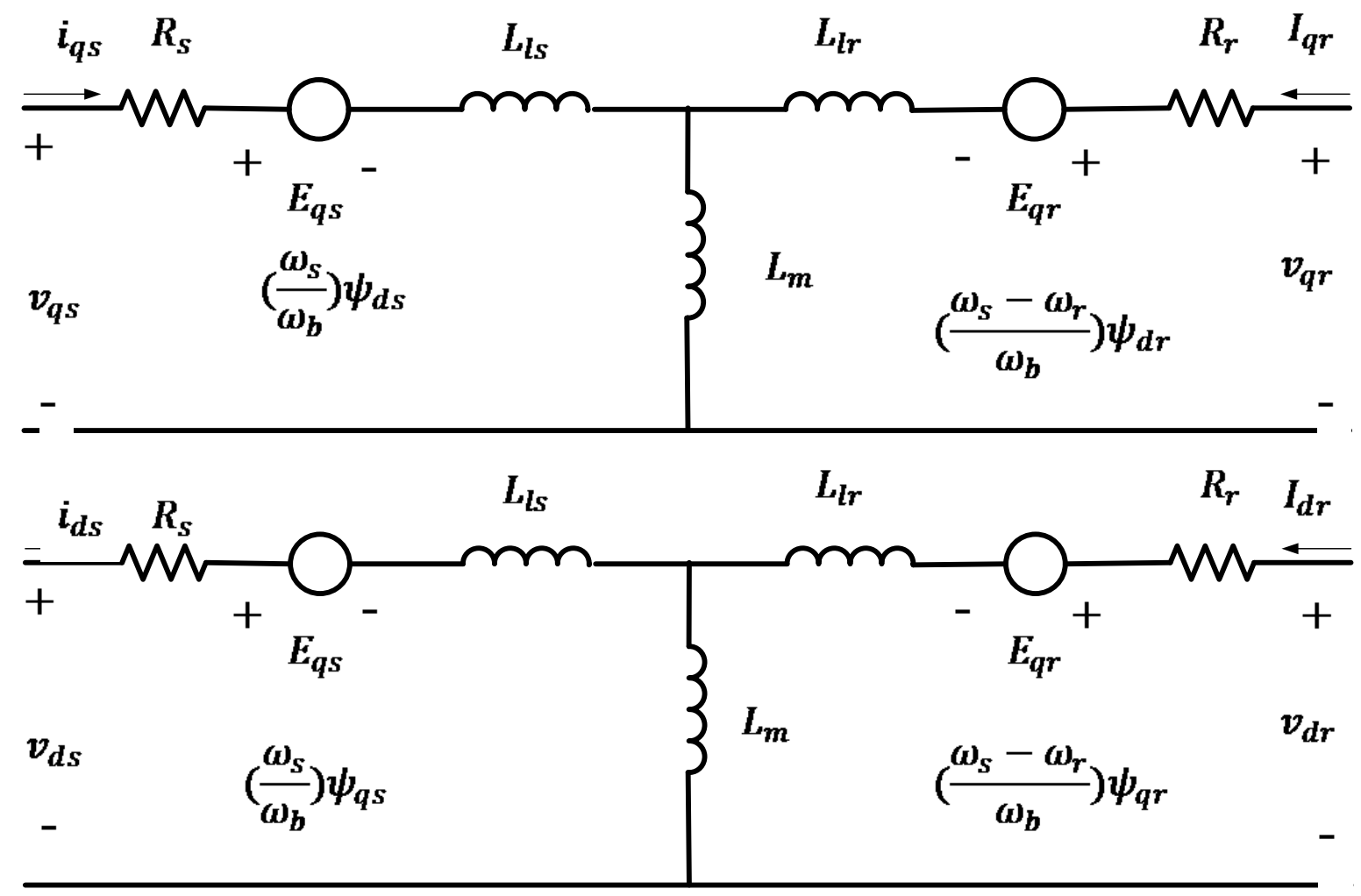

Figure:2.16 Equivalent circuit of DFIG in synchronously rotating reference frame 


\subsubsection{Converter model:}

In this thesis a back to back converter is used. The back to back converter consists of two-level two voltage source converters. One of the converter is connected to the rotor of the DFIG machine and the other converter is connected to the grid through a filter. A DC-link is connected between the two converters as shown in Figure 2.17. In both the converters the switches used are considered to be ideal switches so that the switches allow the flow of power in both the directions. However the switching characteristics of the converters are not discussed as in power system stability studies switching effects and the dynamics of the switching have very less scope and concern, with more concentration on the control strategies used to control the converters.

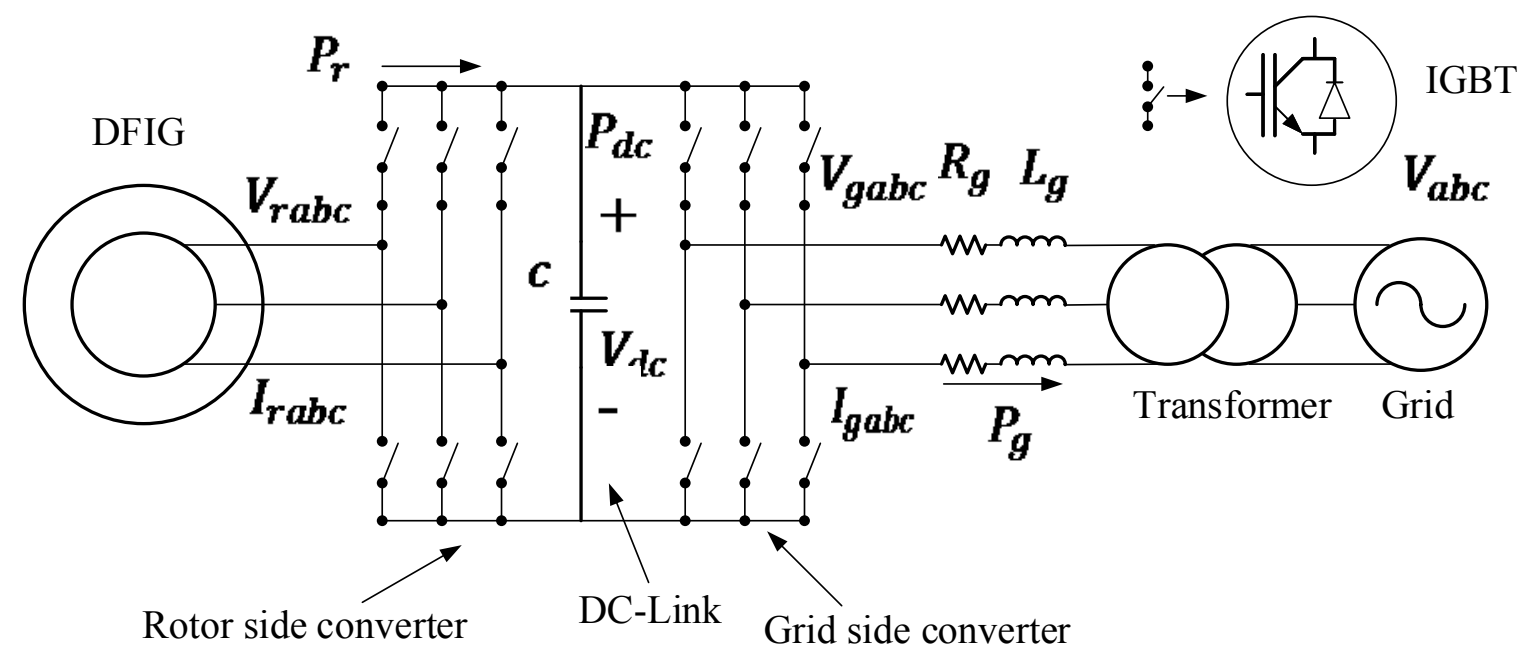

Figure. 2.17: Converter circuit

The converter connected to the rotor of the DFIG machine is termed as rotor side converter and the converter connected to the grid is termed as grid side converter. It can be observed form Figure 2.17, the rotor side converter is connected to grid side converter by the DC link. The switches used and the configuration described above allows the power to flow in both the directions. So, the machine can operate both in sub-synchronous and super-synchronous speed. 
For the converter to function bidirectional the switches which are used are the insulated gate bipolar transistors (IGBT). The switching losses are not considered in this research work. The main objective of these converters is to keep the system stable and supply, and for this the control strategies are applied to each converter separately. The generated control signals from the controllers are fed to the converters using a sinusoidal pulse width modulator. The control strategies for the converters are discussed in Chapter 3.

Active power from grid side converter in $q d$ reference frame is given as:

$$
P_{g}=\left(v_{d g} i_{d g}+v_{q g} i_{q g}\right)
$$

Where $v_{d g}, v_{q g}$ and $i_{d g}, i_{q g}$ are grid side converter voltages and currents respectively.

\section{DC-link model:}

The topics that are usually discussed to model the DC link are, whether to consider the DClink dynamics and regulate the DC voltage. Studies have been conducted by neglecting the DC-link dynamics and by keeping DC-voltage constant [76, 87, and 88]. However in this research work the DC-link voltage is regulated. The main aim of DC-link capacitor is to maintain a constant voltage in its terminals [54].

The power balance equation for the converter can be obtained from Figure 2.17. From (2.64) the power from rotor is given as $P_{r}=\left(v_{d r} i_{d r}+v_{q r} i_{q r}\right)$, similarly from (2.67) the power from grid side converter is given as $P_{g}=\left(v_{d g} i_{d g}+v_{q g} i_{q g}\right)$. From these two models the power balance equations can be given as:

$$
P_{r}=P_{g}+P_{d c}
$$

In the above Figure $2.17 V_{d c}$ is the DC-link voltage and $I_{d c}$ is the DC-link current. Where as $P_{d c}$ is the power from DC-link and $C$ is the capacitance value of the DC-link capacitor. As discussed in the above section, the grid side converter acts as a voltage source converter and it 
maintains the dc-link voltage constant. The details of this converter controller is presented in the next chapter.

The final model used for regulating for DC-link voltage can be written as:

$$
\begin{gathered}
P_{d c}=V_{d c} I_{d c}=C V_{d c} \frac{d V_{d c}}{d t} \\
\frac{d V_{d c}}{d t}=\frac{1}{V_{d c} C}\left(v_{d r} i_{d r}+v_{q r} i_{q r}-\left(v_{d g} i_{d g}+v_{q g} i_{q g}\right)\right)
\end{gathered}
$$

\subsubsection{Grid integrated model:}

Figure 2.18 shows a three phase grid connected wind generation system. In the grid connected wind energy system the stator of the generator and the grid side converter are connected to the grid as shown. The distribution network is represented by equivalent impedance $(Z)$.

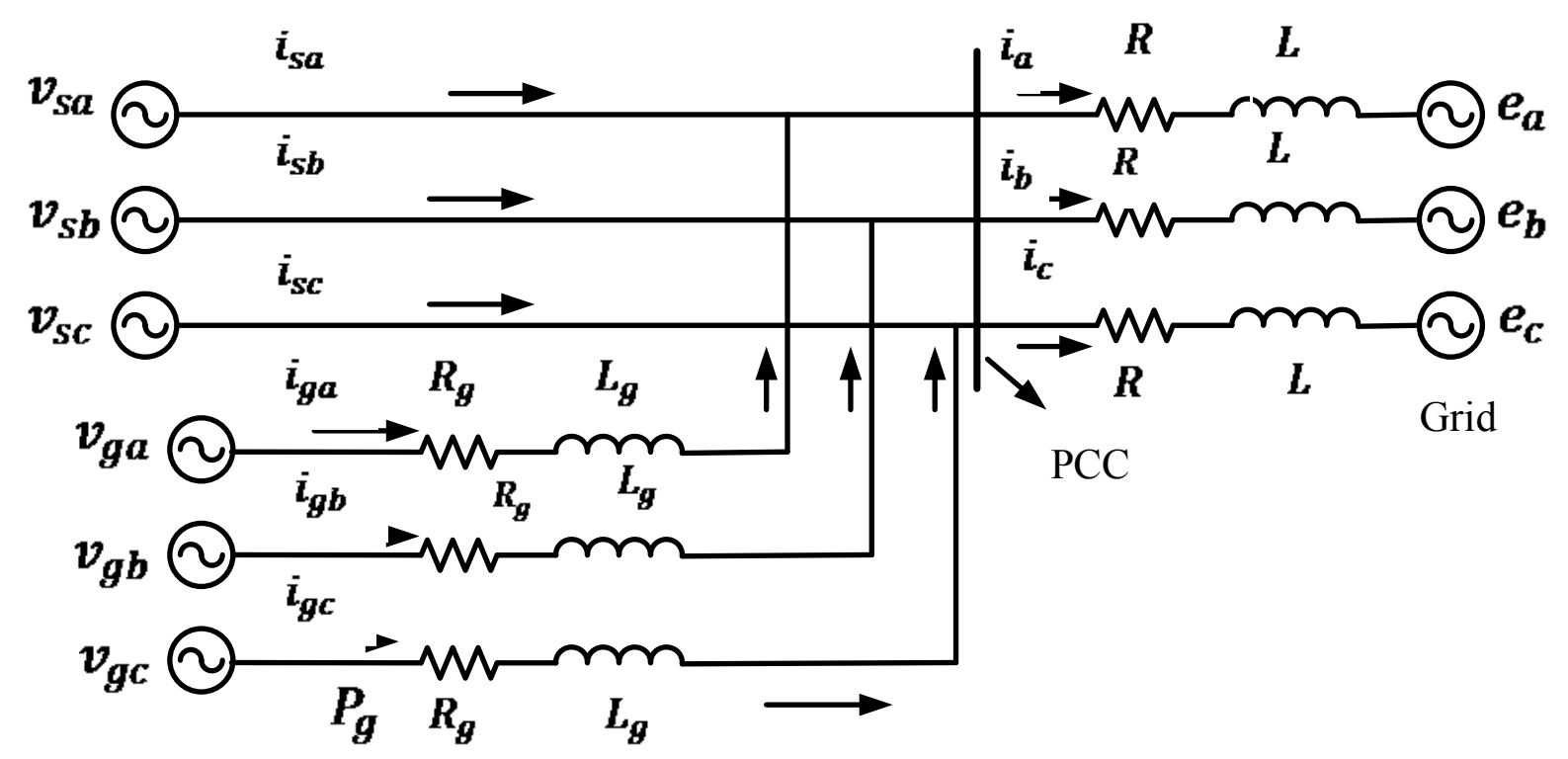

Figure 2.18: Schematic of grid integrated model

In Figure 2.18, $v_{s a}, v_{s b}$ and $v_{s c}$ represent the stator voltages of the generator and $i_{s a}, i_{s b}$ and $i_{s c}$ represent the stator currents. Similarly, $v_{g a}, v_{g b}$ and $v_{g c}$ represent the voltages from the grid side converter and $i_{g a}, i_{g b}$ and $i_{g c}$ represent the currents from the grid side converter. 
$R_{g}$ and $L_{g}$ represent the resistance and inductance of the grid side converter filter respectively. $i_{a}, i_{b}$ and $i_{c}$ are the currents flowing to the grid, whereas $R$ and $L$ are the resistance and inductance of the distribution network respectively. Finally $e_{a}, e_{b}$ and $e_{c}$ are the grid voltages.

The network model in $a b c$ reference frame can be expressed as:

$$
\begin{gathered}
v_{s a}=R i_{a}+L p i_{a}+e_{a} \\
v_{s b}=R i_{b}+L p i_{b}+e_{b} \\
v_{s c}=R i_{c}+L p i_{c}+e_{c} \\
i_{a}=i_{s a}+i_{g a} \\
i_{b}=i_{s b}+i_{g b} \\
i_{c}=i_{s c}+i_{g c} \\
v_{g a}=R_{g} i_{g a}+L_{g} p i_{g a}+v_{s a} \\
v_{g b}=R_{g} i_{g a}+L_{g} p i_{g a}+v_{s a} \\
v_{g c}=R_{g} i_{g a}+L_{g} p i_{g a}+v_{s a}
\end{gathered}
$$

Control and synchronization of the grid parameters in $q d$ reference frame is presented in chapter 3. The distribution network for wind generation system can also be represented with a three phase parallel $R L C$ load. The model with parallel $R L C$ load is presented in Appendix A.

\subsection{Summary:}

In this chapter, a grid integrated DFIG based wind system is first introduced by presenting the classification of wind generation systems. As explained, the system is classified in to four modules and all the modules are discussed clearly in this chapter, this gives a clear idea and description about the different types of wind generation system. Then the mathematical modeling of each module that is used in the rest of the research work is discussed, here the 
controllers are not presented. The control strategies are developed in the next chapter by utilizing the mathematical model. The chapter ends with a summary of all the equations derived.

\subsection{Summary of large signal equations used for analysis:}

Aerodynamic model:

$$
\begin{aligned}
& P_{t}=0.5 \rho A V_{w}^{3} C_{p}(\lambda, \theta) \\
& \lambda=\left(\omega_{t} R\right) /\left(V_{w}\right)
\end{aligned}
$$

Drive train model

$$
\begin{gathered}
2 H_{t} p \omega_{t}=T_{m}-D_{t} \omega_{t}-D_{t g}\left(\omega_{t}-\omega_{r}\right)-T_{t g} \\
2 H_{g} p \omega_{r}=D_{t g}\left(\omega_{t}-\omega_{r}\right)+T_{t g}-D_{g} \omega_{r}-T_{e} \\
p T_{t g}=K_{t g}\left(\omega_{t}-\omega_{r}\right) \\
T_{m}=\frac{P_{t}}{\omega_{t}}
\end{gathered}
$$

Electrical generator model in $a b c$ reference frame

$$
\begin{aligned}
& v_{a s}=R_{s} i_{a s}+\frac{d \psi_{a s}}{d t} \\
& v_{b s}=R_{s} i_{b s}+\frac{d \psi_{b s}}{d t} \\
& v_{c s}=R_{s} i_{c s}+\frac{d \psi_{c s}}{d t} \\
& v_{a r}=R_{r} i_{a r}+\frac{d \psi_{a r}}{d t} \\
& v_{b r}=R_{r} i_{b r}+\frac{d \psi_{b r}}{d t} \\
& v_{c r}=R_{r} i_{c r}+\frac{d \psi_{c r}}{d t}
\end{aligned}
$$




$$
\begin{aligned}
& \psi_{a b c s}=L_{s s} i_{a b c s}+L_{s r} i_{a b c r} \\
& \psi_{a b c r}=L_{r r} i_{a b c r}+L_{r s} i_{a b c s}
\end{aligned}
$$

Electrical generator model in $d q$ reference frame:

$$
\begin{gathered}
v_{d s}=r_{s} i_{d s}-\frac{\omega_{s}}{\omega_{b}} \psi_{q s}+p \psi_{d s} \\
v_{q s}=r_{s} i_{q s}+\frac{\omega_{s}}{\omega_{b}} \psi_{d s}+p \psi_{q s} \\
v_{d r}=r_{r} i_{d r}-\left(\frac{\omega_{s}-\omega_{r}}{\omega_{b}}\right) \psi_{q r}+p \psi_{d r} \\
v_{q r}=r_{r} i_{q r}+\left(\frac{\omega_{s}-\omega_{r}}{\omega_{b}}\right) \psi_{d r}+p \psi_{q r} \\
\psi_{d s}=L_{s} i_{d s}+L_{m} i_{d r} \\
\psi_{q s}=L_{s} i_{q s}+L_{m} i_{q r} \\
\psi_{d r}=L_{r} i_{d r}+L_{m} i_{d s} \\
\psi_{q r}=L_{r} i_{q r}+L_{m} i_{q s} \\
L_{s}=L_{l s}+L_{m} \\
Q_{r}=\frac{3}{2}\left(v_{q r} i_{d r}-v_{d r} i_{q r}\right) \\
T_{e}=L_{m}\left(i_{q s} i_{d r}-i_{d s} i_{q r}\right) \\
P_{s}=\frac{3}{2}\left(v_{d s} i_{d s}+v_{q s} i_{q s}\right) \\
Q_{s}=\frac{3}{2}\left(v_{q s} i_{d s}-v_{d s} i_{q s}\right) \\
\left.P_{d r} i_{d r}+v_{q r} i_{q r}\right) \\
L_{r}
\end{gathered}
$$

DC-Link model: 


$$
\begin{gathered}
P_{d c}=V_{d c} I_{d c}=C V_{d c} \frac{d V_{d c}}{d t} \\
\frac{d V_{d c}}{d t}=\frac{1}{V_{d c} C}\left(v_{d r} i_{d r}+v_{q r} i_{q r}-\left(v_{d g} i_{d g}+v_{q g} i_{q g}\right)\right)
\end{gathered}
$$

The network model in $a b c$ reference frame can be expressed as:

$$
\begin{gathered}
v_{s a}=R i_{a}+L p i_{a}+e_{a} \\
v_{s b}=R i_{b}+L p i_{b}+e_{b} \\
v_{s c}=R i_{c}+L p i_{c}+e_{c} \\
i_{a}=i_{s a}+i_{g a} \\
i_{b}=i_{s b}+i_{g b} \\
i_{c}=i_{s c}+i_{g c} \\
v_{g a}=R_{g} i_{g a}+L_{g} p i_{g a}+v_{s a} \\
v_{g b}=R_{g} i_{g a}+L_{g} p i_{g a}+v_{s a} \\
v_{g c}=R_{g} i_{g a}+L_{g} p i_{g a}+v_{s a}
\end{gathered}
$$




\section{Chapter 3: Controller Design for a DFIG based Wind Energy Generation}

\section{System}

\subsection{Introduction:}

The DFIG based wind generation system has multiple control strategies with different objectives for each controller. The main objective of these control system is, to ensure the safe operation of the mechanical and electrical components in the system by extracting maximum efficiency from the wind turbine. The controller model in the wind turbine generation system can be explained by dividing them into mechanical controller and electrical controller. In this chapter, the control strategies are exposed by relating them to the mathematical models discussed in Chapter 2.

\subsection{Design of Mechanical Controller:}

The main objective of the mechanical controller is to extract the maximum amount of available power from the available wind. It maintains the turbine speed within the allowable limits. The mechanical controller will activate the pitch controller to operate the system within the rated power. The mechanical model with the pitch angle controller used in Simulink is shown in Figure 3.1[89].

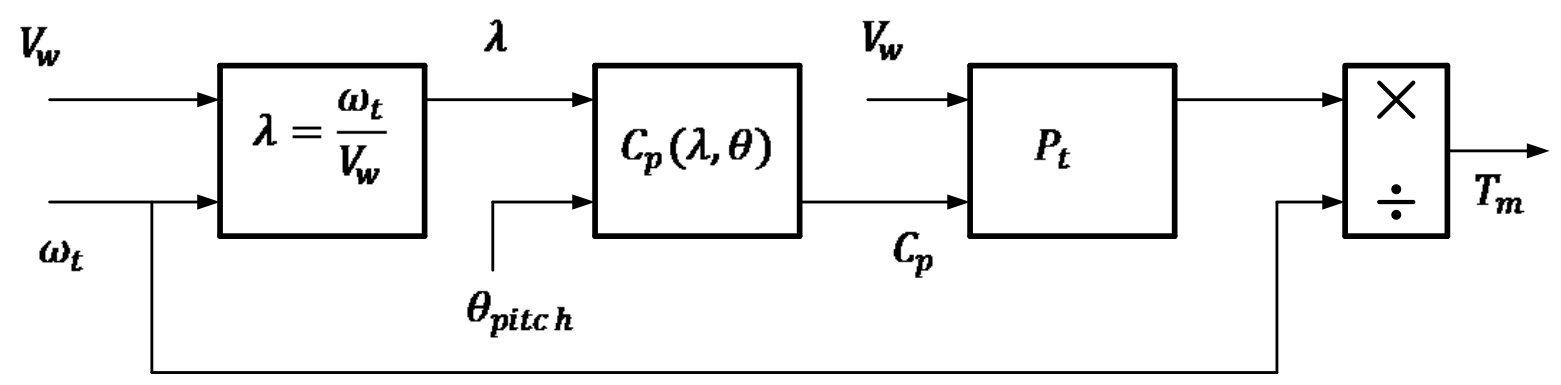

Figure 3.1: Wind turbine model

The input to the model are wind speed $V_{w}$ and mechanical turbine speed $\omega_{t}$; and the final output from the model is the mechanical torque $T_{m}$, this is the input to the drive train model. 
The main controllable command in the turbine model is the pitch angle $\theta_{\text {pitch }}$, this is the input to $C_{p}(\lambda, \theta)$ curve block along with the tip speed ratio $(\lambda)$ command.

\subsubsection{Pitch controller design:}

The main objective of the pitch controller is, to maintain the mechanical power that is delivered to the shaft within the limits, this is possible by pitching the blades. When the wind speed or the power extracted from the wind is above the rated power the pitch command is activated, and the blades are pitched to reduce the mechanical power $\left(P_{t}\right)$ delivered to the shaft. During the maximum wind speed the pitch angle is increased. Furthermore, when the wind power is below the rated power, the pitch angle is controlled by maintaining the pitch at its minimum, this makes the turbine to operate at its $C_{\text {pmax }}$. The pitch controller used in the research work is as shown in Figure 3.2[68]. The parameters for the control block shown in Figure 3.2 are presented in appendix B.

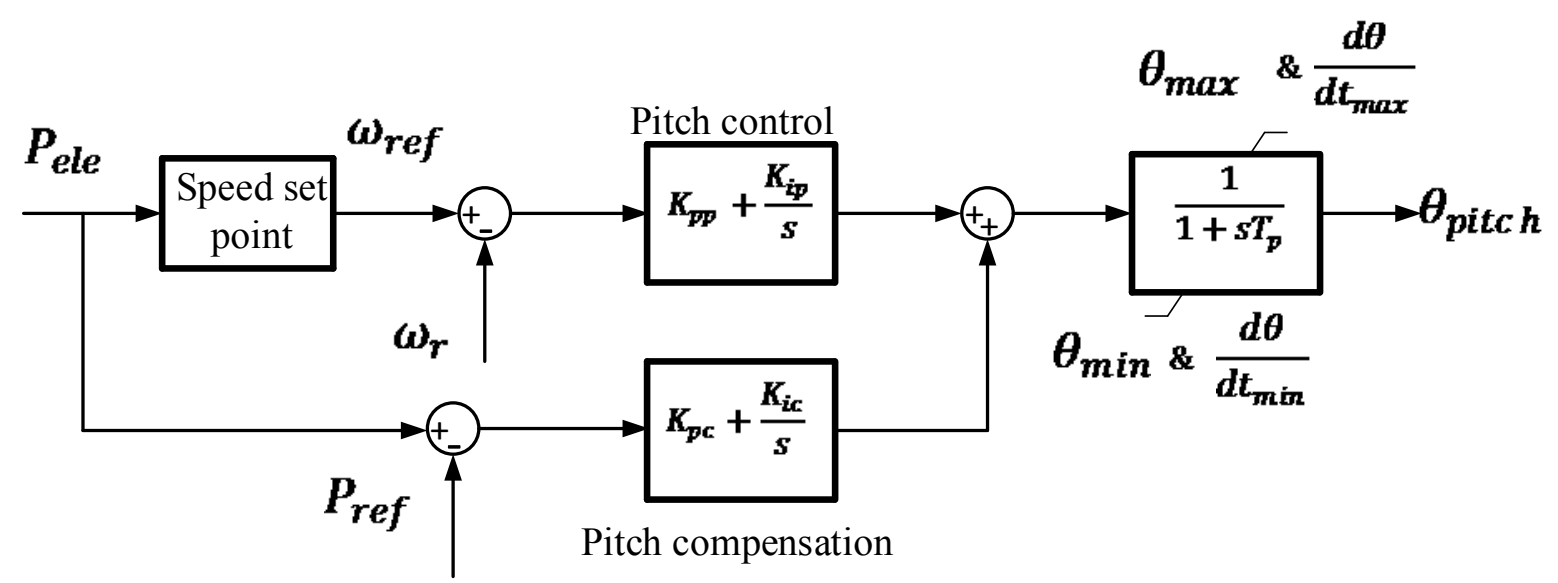

Figure 3.2: Pitch controller

From the above discussion it can be concluded that during reduced wind and maximum wind speeds the power output of the wind system is controlled by using the pitch controller. In Figure 3.2, it can be observed that there are three modes of control. The controller has the rotor speed control, the pitch angle control and the power compensation. In Figure 3.2 above $P_{\text {ele }}$ 
is the electrical power supplied to the grid, $P_{r e f}$ is the reference active power. $\omega_{r e f}$ and $\omega_{r}$ are the rotor reference speed and rotor speed respectively. $\omega_{\text {ref }}$ is generally $1.2 p u$. But for lesser wind speeds, i.e. when the power below $0.7 \mathrm{pu}$, the reference speed is reduced according to $3.1[68]$ and finally $\theta_{p i t c h}$ is the pitch angle command given to $C_{p}(\lambda, \theta)$ block in Figure 3.1.

$$
\omega_{\text {ref }}=-0.67\left(P_{\text {ele }}\right)^{2}+1.42(P)+0.51
$$

The other mechanical control design is based on the drive train model.

\subsubsection{Drive train design:}

The input to the drive train are, the mechanical torque from the wind turbine rotor and the electrical torque from the generator. The model for drive train is given in Chapter 2.

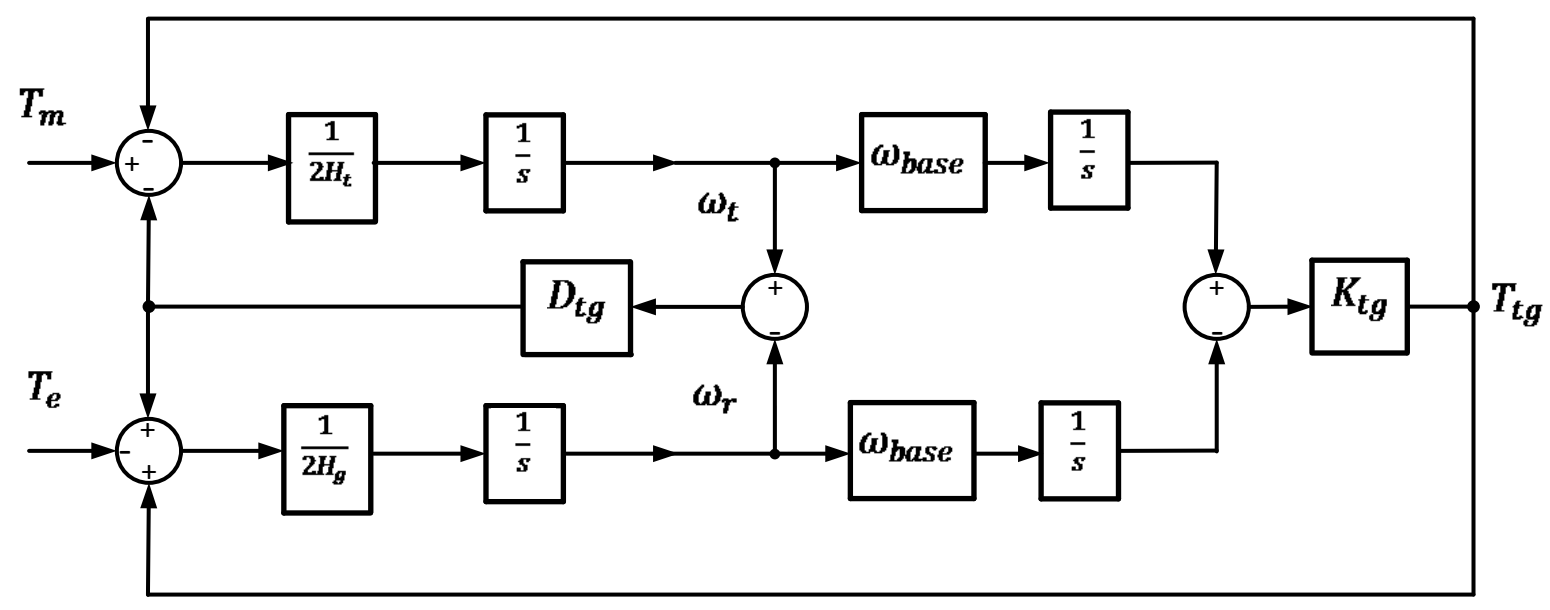

Figure 3.3: Drive train physical model

In this chapter the design of drive train implemented in Simulink is discussed. The block diagram for two mass drive train used in Simulink is as shown in Figure 3.3[68]. In Figure 3.3, $T_{m}$ is the mechanical torque from the aerodynamic model; $T_{e}$ is the electrical torque form the generator; $\omega_{t}$ and $\omega_{r}$ are the wind turbine rotor speed and generator rotor speed respectively; $H_{t}$ and $H_{g}$ are the inertia constants of wind turbine rotor and generator respectively; $T_{t g}$ is the shaft torque; $D_{t g}$ is the damping coefficient and $K_{t g}$ is the shaft stiffness. It can be observed 
from the Figure 3.3, that the speed of the wind turbine and the rotor speed of the generator are dependent on the wind power model and the electrical power from the generator.

\subsection{Design of Electrical Controller:}

Modeling of the electrical generator and the grid are discussed in Chapter 2, it can be observed from Figure 2.6 that, these electrical components are connected by a back to back converter. The control schemes are applied to the converters discussed in Chapter 2, which controls the grid and generator parameters. The control of the converters is done in the synchronously rotating reference frame, the same reference frame which is used for developing the mathematical model for the electrical machine. The two converters mentioned are the rotor side converter and the grid side converter, and the control schemes are applied to these two converters and they are explained in the subsection of this section. The two control schemes of the converters can be termed as:

a) Rotor side converter controller

b) Grid side converter controller

\section{Control strategies:}

To develop the control strategies for the converters any one of the axes in two reference frame $(q d)$, rotating with synchronous speed is aligned with either the flux or the voltages of the stator. The two commonly used control strategies are the stator flux oriented control and the stator voltage oriented control. In the stator flux oriented control the $d$-axis is aligned with the stator flux linkage vector [63]. This results in $\psi_{d s}=\psi_{s}$ and $\psi_{q s}=0$. Whereas in stator voltage oriented control, the $d$-axis is aligned with the stator voltage linkage vector, resulting in $V_{d s}=V_{s}$ and $V_{q s}=0$. Usually for rotor side converter control the stator flux oriented control is used and for grid side converter controller stator voltage oriented control is adopted 
[63]. In this thesis the stator voltage oriented control is implemented for both the rotor side converter controller and grid side converter controller as discussed in [89]. The main objective of these control strategies is to decouple the control of active and reactive power, and the control is possible by using both of the strategies [89].

The controllers are designed based on stator voltage oriented control which yields:

$$
V_{d s}=V_{s} ; V_{q s}=0
$$

\subsubsection{Rotor side converter controller:}

The main objective of the rotor side converter controller is to control both the active and reactive power of the stator. It consists of two control loops; the inner control loop regulates the $d$ and $q$ axis rotor currents, whereas the outer loop controls the active $P_{S}$ and reactive $Q_{s}$ power of the generator stator. This can be observed from the following mathematical modeling derived based on (3.2) and (3.3). By neglecting the transients in the stator flux linkages of (2.52) and (2.53) the obtained stator voltage equations are $v_{d s}=r_{s} i_{d s}-\omega_{s} \psi_{d s}$ and $v_{q s}=$ $r_{s} i_{q s}+\omega_{s} \psi_{q s}$. From the obtained voltage equations, in stator voltage orientation the resultant flux linkages under an assumption that resistive drops are negligible are:

$$
\psi_{d s}=0 ; \psi_{q s}=-\frac{V_{d s}}{\omega_{s}}
$$

By applying (3.2) and (3.3) to the stator power equation (2.62) the obtained power equation is $P_{s}=-\frac{L_{m}}{L_{s}}\left(v_{d s} i_{d r}\right)$; from the obtained power model it can be observed that it is independent from $q$ - axis rotor current and it is only dependent on the $d$ - axis rotor current. By applying the same (3.2) and (3.3) to stator reactive power model as given in (2.63), the obtained rotor model is $Q_{s}=\frac{\left(v_{d s}\right)^{2}}{\omega_{s} L_{s}}+\frac{L_{m}}{L_{s}}\left(v_{d s} i_{q r}\right)$; from this model it can be observed that stator reactive power is totally depending on the $q$ - axis rotor current and it is independent from $d$-axis rotor 
current. From this discussion it can be concluded that the stator active and reactive power can be controlled independently by rotor $d$ - axis and $q$ - axis currents respectively. The control of stator active and reactive power gives reference current outputs $i_{d r}^{*}$ and $i_{q r}^{*}$ respectively.

\section{Design for rotor inner current control loop:}

The generated reference currents are fed as inputs to the inner current loops of the rotor current controller. The final outputs of the rotor side converter controller are $v_{d r}$ and $v_{q r}$. The controller is designed based on the rotor voltage models developed in Chapter 2 i.e. (2.54) and (2.55) which can be expanded and expressed as:

$$
\begin{aligned}
& v_{d r}=r_{r} i_{d r}-\left(\omega_{s}-\omega_{r}\right)\left(L_{r} i_{q r}+L_{m} i_{q s}\right)+p\left(L_{r} i_{d r}+L_{m} i_{d s}\right) \\
& v_{q r}=r_{r} i_{q r}+\left(\omega_{s}-\omega_{r}\right)\left(L_{r} i_{d r}+L_{m} i_{d s}\right)+p\left(L_{r} i_{q r}+L_{m} i_{q s}\right)
\end{aligned}
$$

While designing the rotor side controller in (3.4) and (3.5) the second terms on right hand side acts as the decoupling elements i.e. $-\left(\omega_{s}-\omega_{r}\right)\left(L_{r} i_{q r}+L_{m} i_{q s}\right)$ and $\left(\omega_{s}-\omega_{r}\right)\left(L_{r} i_{d r}+\right.$ $\left.L_{m} i_{d s}\right)$; if the transients of the machine are neglected the derivative terms become zero. So by equating the derivative terms zeros the terms obtained for $d$-axis rotor voltage will be in terms of $i_{d r}$; similarly the $d$ - axis rotor voltage will be in terms of $i_{q r}$ and they are given as $v_{d r 1}=$ $r_{r} i_{d r}+\left(L_{r}-\frac{L_{m}^{2}}{L_{s}}\right) p i_{d r}$ and $v_{q r 1}=r_{r} i_{q r}+\left(L_{r}-\frac{L_{m}^{2}}{L_{s}}\right) p i_{q r}$.From these equations a transfer function can be developed for the inner current control loops and from this the proportional gain and integral gain can be obtained easily. It can be observed from (3.6) and (3.6) that the design is same for both the control loops so the PI control design is also same for both the inner current control loops. In these control loops $v_{d r 1}$ and $v_{q r 1}$ are the outputs of the rotor current controller loops and they are fed to the pulse width modulator of the converter along with the decoupled elements. Finally these obtained signals from the modulator are fed to the converter circuit and this is connected to the DC link of the model. In some of the cases the 
rotor resistances are fed to the controller along with the decoupled elements. The obtained transfer function is:

$$
\begin{aligned}
& \frac{i_{d r}}{v_{d r 1}}=\frac{1}{r_{r}+\left(L_{r}-\frac{L_{m}^{2}}{L_{s}}\right) s} \\
& \frac{i_{q r}}{v_{q r 1}}=\frac{1}{r_{r}+\left(L_{r}-\frac{L_{m}^{2}}{L_{s}}\right) s}
\end{aligned}
$$

The rotor side converter controller block diagram is described in Figure 3.4:

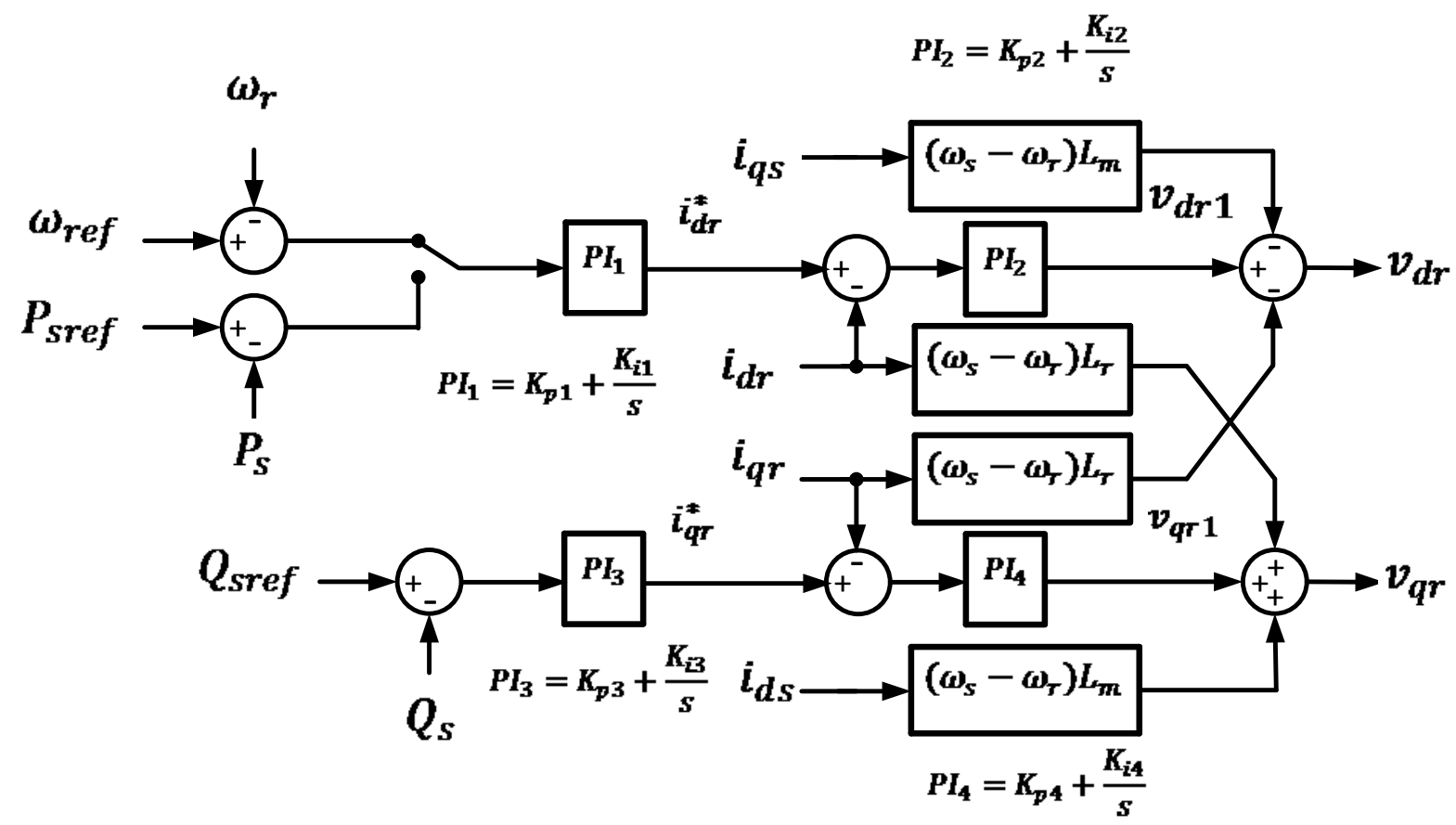

Figure 3.4: Rotor side converter controller block

In Figure 3.4; $i_{d r}$ and $i_{q r}$ are the rotor currents and they are transformed from rotor three phase currents $i_{r a b c}$ to $i_{r d q}$ by applying a transforming angle of $\left(\theta_{s}-\theta_{r}\right)$ where $\theta_{s}$ is the angle obtained from $v_{s a b c}$ at grid frequency and $\theta_{r}$ is the rotor angle. These angles are transformed by using a simple phase locked loop which is discussed while designing of grid controller. Similarly in Figure 3.4; $P_{S}$ is the stator active power and $Q_{S}$ is the stator reactive power and 
these are obtained from $v_{s a b c}$ and $i_{s a b c}$. Reactive power reference $Q_{s r e f}$ is given as 0 , whereas stator active power reference $P_{\text {sref }}$ is generated from maximum power tracking method, in this thesis a simple look up table is used which tries to obtain the reference stator power by plotting for power and speed. The other reference at $v_{d r}$ control is the generator reference value which is set by the speed set point discussed in Section 3.2. Nonetheless, any of the method can be used for $v_{d r}$ control.

\subsubsection{Grid side converter controller:}

To design the grid side converter controller first the grid model in the $a b c$ reference frame developed in Chapter 2 are transformed into two reference frame. This is possible by using a phase locked loop which provides required transformation angle and the frequency for synchronizing the model with the grid. The design and operation of PLL is derived from [51] and it is shown in Figure 3.5.

\section{Design of grid controller:}

The grid model discussed in Chapter 2 in $a b c$ reference frame is transformed to $d q$ reference frame based on the following transformation matrix [51]:

$$
\left[\begin{array}{l}
v_{d} \\
v_{q}
\end{array}\right]=\frac{2}{3}\left[\begin{array}{ccc}
\cos \theta & \cos \left(\theta-\frac{2 \pi}{3}\right) & \cos \left(\theta+\frac{2 \pi}{3}\right) \\
-\sin \theta & -\sin \left(\theta-\frac{2 \pi}{3}\right) & -\sin \left(\theta+\frac{2 \pi}{3}\right)
\end{array}\right]\left[\begin{array}{l}
v_{a} \\
v_{b} \\
v_{c}
\end{array}\right]
$$

In (3.8); $\theta$ is the transformation angle in the above matrix is the grid side converter terminal voltage angle and it is expressed as $\theta=\omega_{0} t+\delta$; where $\delta$ is given as $p \delta=\omega-\omega_{0}$ where $\omega$ is the grid side convert terminal frequency. The measured phase angle by PLL is given as $\theta_{p l l}$ and the measured frequency is given as $\omega_{p l l}$ and this provides the transformation angle and the frequency to the grid side convert controller design. The obtained angle and frequency information is used for both $a b c-d q$ and $d q-a b c$ transformation at the grid end. Initially 
for operating PLL the $q$ - axis voltage of stator $v_{q s}$ is obtained by $a b c-d q$ transformation technique. Once the grid side converter terminal voltage is transformed and the transformation angle is $\theta_{p l l}$ then the obtained equation is $v_{q s}=V \sin \left(\theta-\theta_{p l l}\right)=V \sin \left(\delta-\delta_{p l l}\right)$; where $\delta_{p l l}$ is the measured phase angle and it is expressed as $\delta_{p l l}=\theta_{p l l}-\omega_{0} t$.

The grid side voltage frequency $\omega_{p l l}$ is obtained by the addition of the error signal processed in the PI controller and $\omega_{0}$; and it is given as:

$$
\begin{gathered}
\omega_{p l l}=\left(k_{p p l l}+\frac{k_{i p l l}}{s}\right) v_{q s}+\omega_{0} \\
p \delta_{p l l}=\omega_{p l l}-\omega_{0}
\end{gathered}
$$

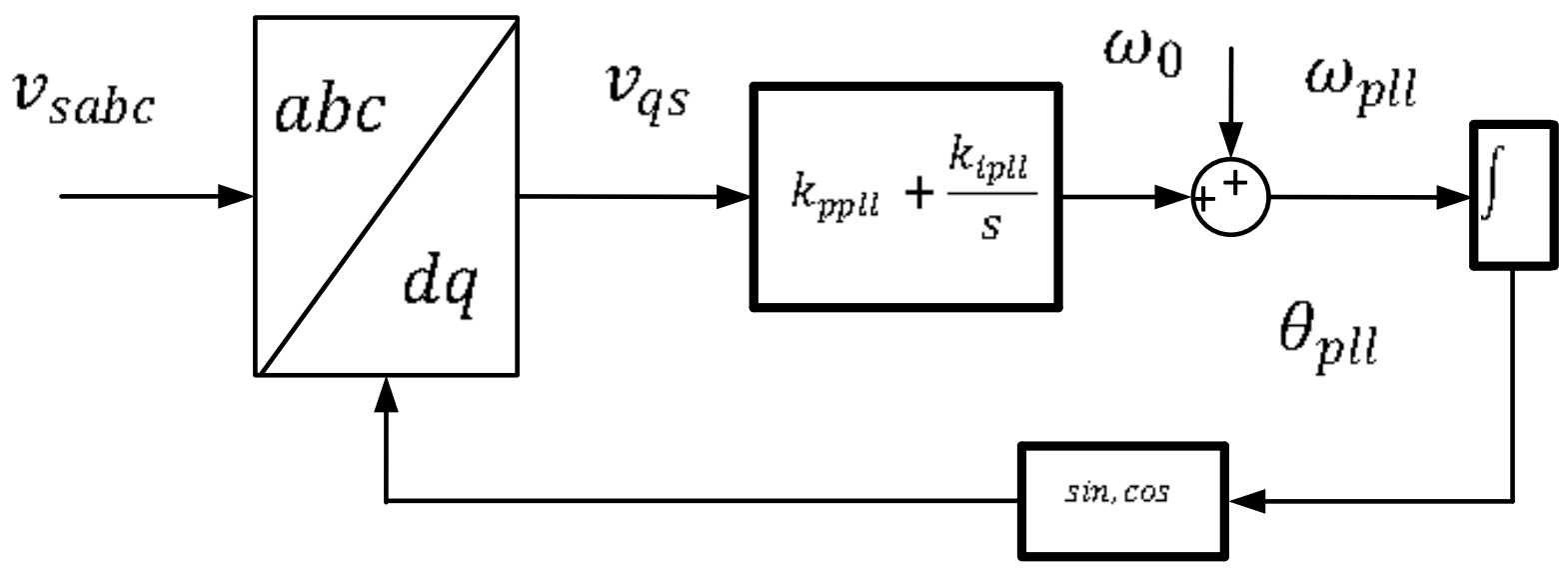

Figure 3.5: PLL model

By using this three phase PLL the grid equations can be transformed in to two reference frame. The $d q$ transformed equations of the grid model discussed in Section 2.4.4 are as follows:

$$
\begin{aligned}
& v_{d s}=r i_{d}+L p i_{d}-\omega_{p l l} L i_{q}+e_{d} \\
& v_{q s}=r i_{q}+L p i_{q}-\omega_{p l l} L i_{d}+e_{q}
\end{aligned}
$$

In (3.11) and (3.12) $v_{d s}$ and $v_{q s}$ are the stator voltages; $r$ and $L$ are the grid filter resistance and inductance respectively; $i_{d}$ and $i_{q}$ are the total currents supplied to the grid in $d q$ reference frame. Finally $e_{d}$ and $e_{q}$ are the transformed voltages of the grid terminal. The model for the 
total current supplied to the grid i.e. $i_{d}$ and $i_{q}$ are given as the sum of the stator currents $i_{d q s}$ and grid side converter currents $i_{d q g}$ :

$$
\begin{aligned}
& i_{d}=i_{d s}+i_{d g} \\
& i_{q}=i_{q s}+i_{q g}
\end{aligned}
$$

The grid side converter voltages in $d q$ reference frame can be expressed as:

$$
\begin{aligned}
& v_{d g}=R_{g} i_{d g}+L_{g} p i_{d g}-\omega_{p l l} L_{g} i_{q g}+v_{d s} \\
& v_{q g}=R_{g} i_{q g}+L_{g} p i_{q g}+\omega_{p l l} L_{g} i_{d g}+v_{q s}
\end{aligned}
$$

Finally the grid voltages are expressed as:

$$
\begin{gathered}
e_{d}=E \cos \left(\delta_{p l l}\right) \\
e_{q}=-E \sin \left(\delta_{p l l}\right)
\end{gathered}
$$

From the above derived grid side model the grid side converter controller can be designed.

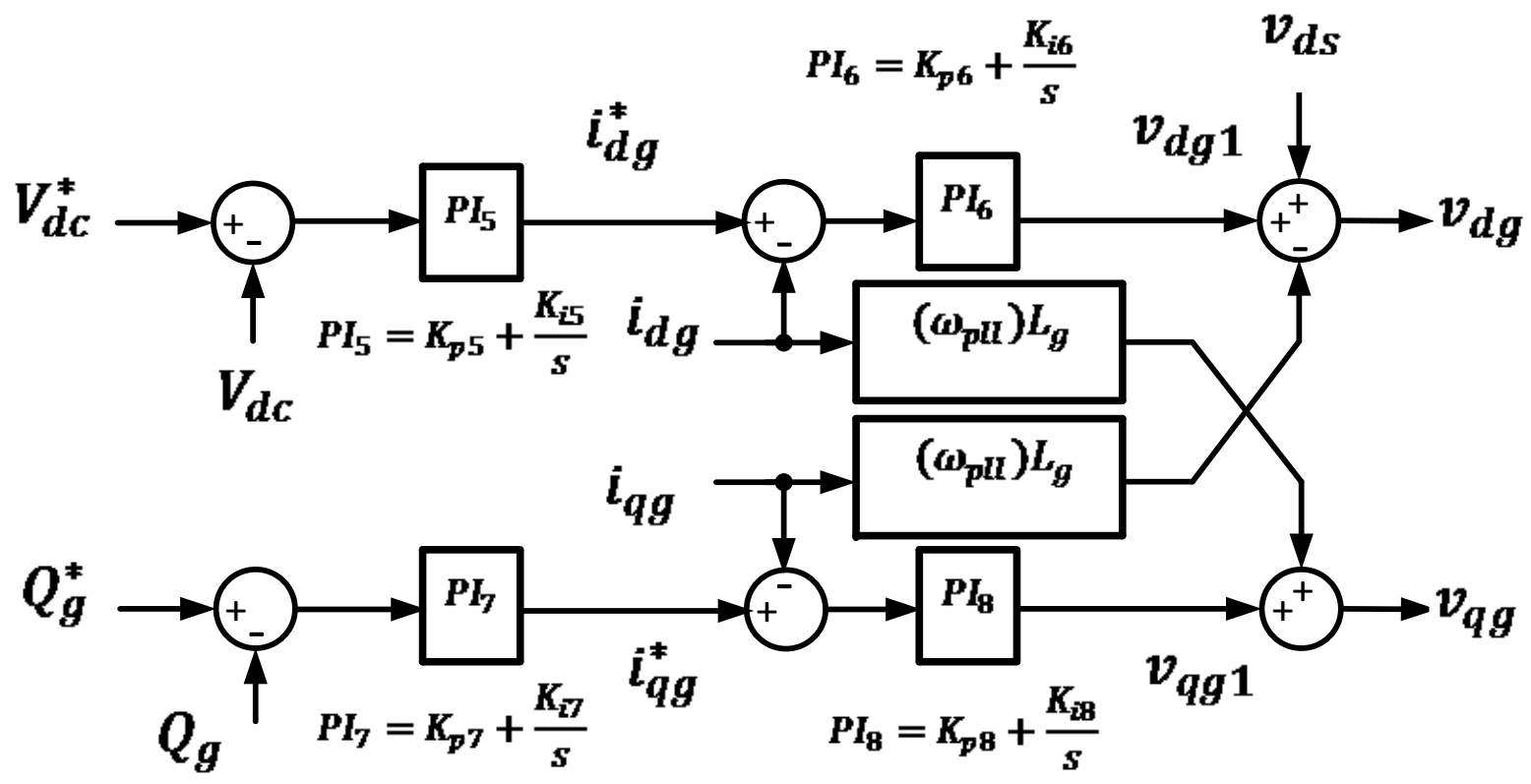

Figure 3.6: Grid side converter controller

The grid side converter controller used in this thesis work is as shown in Figure 3.6[63]. The main objective of this grid side converter controller is to regulate the DC-link voltage and 
exchange the power between the rotor side converter and the grid. The other objective of this controller is to control the reactive power that is delivered to the grid, at the grid side converter. Similar to that of the rotor side converter controller the grid side converter controller also consists of two cascaded control loops. The DC-link voltage and the reactive power are controlled by the outer control loop whereas the inner current control loop regulates the current components in the grid side converter.

From the above discussed grid model the active power and the reactive power at the grid can be expressed as:

$$
\begin{aligned}
& P_{g c}=\frac{3}{2}\left(v_{d s} i_{d g}+v_{q s} i_{q g}\right) \\
& Q_{g}=\frac{3}{2}\left(v_{q s} i_{d g}-v_{d s} i_{q g}\right)
\end{aligned}
$$

In these equation by applying the synchronously rotating reference frame and aligning the $d$ axis on the grid voltage vector the obtained results are $v_{d s}=v_{s}$ and $v_{q s}=0$. By applying this to (3.15) and (3.16) the obtained equations are:

$$
\begin{gathered}
v_{d g}=R_{g} i_{d g}+L_{g} p i_{d g}-\omega_{p l l} L_{g} i_{q g}+v_{d s} \\
v_{q g}=R_{g} i_{q g}+L_{g} p i_{q g}+\omega_{p l l} L_{g} i_{d g}
\end{gathered}
$$

From (3.19) and (3.20) the outer control loops can be designed, the obtained result is a function of DC-link voltage $v_{d c}$ and the grid side converter current $i_{d g}[63,89]$. By using this independent control loop is developed for DC-link voltage $v_{d c}$ with $i_{d g}^{*}$ as its output. Similarly the grid side reactive power also can be controlled individually, this can be observed from the equation (3.20). When the reference frame is applied to (3.20) the obtained result is $Q_{g}=$ $\frac{3}{2}\left(-v_{d s} i_{q g}\right)$. With this the outer control loop, for the grid side converter the control strategies for the outer loop can be designed easily. From this outer loop $i_{q g}^{*}$ is obtained. Similarly for 
the inner control loop design the same reference frame is applied. The inner control loops are mainly designed as current control loops which uses grid side currents as inputs.

\section{Grid side inner current control loop:}

With (3.21) and (3.22) the inner current control loop can be designed. In (3.21) $\omega_{p l l} L_{g} i_{q g}$ and $v_{d s}$ are fed as the inputs at the outer end of the controller design and in (3.22) $\omega_{p l l} L_{g} i_{d g}$ is fed at the outer end. $\omega_{p l l} L_{g} i_{q g}$ and $\omega_{p l l} L_{g} i_{d g}$ act as decoupling elements for grid side converter controller. The final obtained equations are:

$$
\begin{aligned}
& v_{d g}=R_{g} i_{d g}+L_{g} p i_{d g} \\
& v_{q g}=R_{g} i_{q g}+L_{g} p i_{q g}
\end{aligned}
$$

From (3.23) and (3.24) the PI controller can be designed and it can be observed that both the controllers are same.

\subsection{Summary:}

In this chapter, the designs of the electrical and mechanical controllers are discussed. When designing the electrical controllers the inner current control loop and the outer loop designs are also considered for both RSC and GSC. All the parameters of the model are presented in Appendix B. The mathematical model of the expanded controller with proportional and integral gain is not shown, and it is discussed in Chapter 4. In Chapter 4 the small signal and the large signal analyses are discussed for the models developed in Chapter 2 and the controllers discussed in Chapter 3.

\subsection{Summary of large signal equations of controllers from chapter 3:}

$$
\omega_{p l l}=\left(k_{p p l l}+\frac{k_{i p l l}}{s}\right) v_{q s}+\omega_{0}
$$




$$
\begin{gathered}
p \delta_{p l l}=\omega_{p l l}-\omega_{0} \\
v_{d s}=r i_{d}+L p i_{d}-\omega_{p l l} L i_{q}+e_{d} \\
v_{q s}=r i_{q}+L p i_{q}-\omega_{p l l} L i_{d}+e_{q} \\
i_{d}=i_{d s}+i_{d g} \\
i_{q}=i_{q s}+i_{q g} \\
v_{d g}=R_{g} i_{d g}+L_{g} p i_{d g}-\omega_{p l l} L_{g} i_{q g}+v_{d s} \\
v_{q g}=R_{g} i_{q g}+L_{g} p i_{q g}+\omega_{p l l} L_{g} i_{d g}+v_{q s} \\
e_{d}=E c o s\left(\delta_{p l l}\right) \\
e_{q}=-E s i n\left(\delta_{p l l}\right) \\
v_{d g}=R_{g} i_{d g}+L_{g} p i_{d g}-\omega_{p l l} L_{g} i_{q g}+v_{d s} \\
v_{q g}=R_{g} i_{q g}+L_{g} p i_{q g}+\omega_{p l l} L_{g} i_{d g} \\
\left.v_{d g}=R_{g} i_{d g}+L_{g} p i_{d g} i_{d g}+v_{q s} i_{q g}\right) \\
v_{q g}=R_{g} i_{q g}+L_{g} p i_{q g} \\
3 \\
\frac{3}{2}\left(v_{q s} i_{d g}-v_{d s} i_{q g}\right)
\end{gathered}
$$




\section{Chapter 4: Performance Analysis of a Grid Connected DFIG based Wind}

\section{Generation System}

\subsection{Introduction:}

The mathematical modelling and control strategies of the wind energy conversion system are discussed in Chapter 2 and 3. In this chapter the analysis is carried on the developed system. The main objective of this chapter is to investigate on the stability analysis of the grid integrated wind system. The two analysis which are carried out in this thesis are the

a) Small signal stability analysis and

b) Large signal analysis.

The chapter is organized as follows: In Section 4.2, small signal stability analysis is introduced and the small signal model is developed. The small signal model is developed with controller and without controller by connecting the system to the grid and finally Eigen values and participation factors of $(E, A)$ are derived and a conclusion is made on the sensitivity of the parameters. In Section 4.3 the analysis is executed for the large signal model, this analysis is made for the detailed and averaged models. At last, the system is validated for the large signal model in Section 4.3.

\subsection{Small Signal Stability Analysis:}

In a small signal stability analysis the system is subjected to small disturbances [82]. Small signal stability analysis used in this thesis is totally a mathematical analysis on the developed system and it is used for determining the characteristics of the dynamics of the wind system. The small signal analysis is carried out in the following steps, first the large signal model developed in Chapter 2 and 3 are linearized around an equilibrium point. The linearization is carried out by applying Taylor expansion about an operating point and this is done as described 
in [82 and 84]. These obtained linear differential equation describe the dynamic behaviour of small displacements about the operating point [84]. With this the entire system can be treated as a linear system with small disturbances and further the Eigen analysis can be carried out on the obtained linear model through which the stability of the system can be determined.

This is described clearly in this section which is adopted from [82].

In general the power system is a dynamic system and it consists of nonlinear differential equations. The linear analysis is carried out for the general nonlinear equation which is given as:

$$
\dot{X}=f(X, U)
$$

In (4.1) $X$ is a vector of state variables represented as $X=\left[x_{1}, x_{2} \ldots \ldots x_{n}\right]^{T}$ and $U$ is a vector of input variables given as $U=\left[u_{1}, u_{2} \ldots \ldots u_{n}\right]^{T}$ for $n$ nonlinear equations.

For linearization technique consider initial state vector $x_{0}$ and the corresponding input vector $u_{0}$ at an equilibrium point where the small signal stability analysis is carried out. Then the obtained equation is

$$
\dot{X}_{0}=f\left(X_{0}, U_{0}\right)
$$

If the system is perturbed to small disturbances the obtained equations are:

$$
X=X_{0}+\Delta X ; U=U_{0}+\Delta U
$$

$\Delta$ Represents the small deviation.

Substituting (4.3) in (4.1) the new system obtained is

$$
\dot{X}=\dot{X}_{0}+\Delta \dot{X}=f\left[\left(X_{0}+\Delta X\right),\left(U_{0}+\Delta U\right)\right]
$$

Equilibrium point mentioned above can be defined as the point where all the derivatives of the large signal model are equal to zero; once the obtained equations are solved simultaneously the equilibrium point parameters can be obtained. The equilibrium points describe the points 
on the trajectory with zero velocity [82]. So the equilibrium point will satisfy $f\left(X_{0}\right)=0$. Where $X_{0}$ is the state vector $X$ at the equilibrium point.

The nonlinear function $f(X, U)$ can be expanded by using Taylor's series expansion. Second and higher order terms of $\Delta X$ and $\Delta U$ are neglected [82 and 84]. The obtained expression is:

$$
\begin{gathered}
\dot{X}_{\iota}=f_{i}\left[\left(X_{0}+\Delta X\right),\left(U_{0}+\Delta U\right)\right] \\
\dot{X}_{\iota}=f_{i}\left(X_{0}, U_{0}\right)+\frac{\partial f_{i}}{\partial x_{1}} \Delta x_{1}+\cdots+\frac{\partial f_{i}}{\partial x_{n}} \Delta x_{n}+\frac{\partial f_{i}}{\partial u_{1}} \Delta u_{1}+\cdots+\frac{\partial f_{i}}{\partial u_{r}} \Delta u_{r}
\end{gathered}
$$

From (4.6) the final linearized equation can be obtained in the form of:

$$
\Delta \dot{X}=A \Delta X+B \Delta U
$$

$A$ and $B$ are obtained at equilibrium point.

From the above discussed method the linearized equations of the model in this thesis can be developed and it is derived as follows:

\section{Mechanical model:}

Linearized equations for the mechanical model which consist of the modeling of drive train and the aerodynamic model can be obtained by applying the above method to (2.16) - (2.19); the final equations derived are:

$$
\begin{gathered}
2 H_{t} p\left(\Delta \omega_{t}\right)=-\left(\frac{-P_{t}}{\omega_{t 0}^{2}}-D_{t g}\right)\left(\Delta \omega_{t}\right)-K_{t g} \Delta\left(\theta_{t}-\theta_{r}\right)+D_{t g}\left(\Delta \omega_{r}\right) \\
p \Delta\left(\theta_{t}-\theta_{r}\right)=\left(\Delta \omega_{t}\right)-\left(\Delta \omega_{r}\right) \\
2 H_{g} p\left(\Delta \omega_{r}\right)=K_{t g} \Delta\left(\theta_{t}-\theta_{r}\right)+D_{t g}\left(\Delta \omega_{t}\right)-D_{t g}\left(\Delta \omega_{r}\right)-L_{m} i_{q s 0}\left(\Delta i_{d r}\right)-L_{m} i_{d r 0}\left(\Delta i_{q s}\right) \\
+L_{m} i_{d s 0}\left(\Delta i_{q r}\right)+L_{m} i_{q r 0}\left(\Delta i_{d s}\right)
\end{gathered}
$$

These three equations contribute the linearized model for the drive train and the aerodynamic model. Variables $\left(X_{0}\right)$ denote the constant values at the equilibrium point. All the parameters are discussed in Appendix B. 


\section{DFIG model:}

Linearized equation for the DFIG model are derived from the DFIG large signal model equation (2.52)- (2.61). The final linearized equations are as follows:

$$
\begin{gathered}
\frac{L_{s}}{\omega_{b}} p\left(\Delta i_{d s}\right)+\frac{L_{m}}{\omega_{b}} p\left(\Delta i_{d r}\right)=\left(\Delta v_{d s}\right)+\frac{\omega_{e}}{\omega_{b}} L_{s}\left(\Delta i_{q s}\right)-R_{s}\left(\Delta i_{d s}\right)+\frac{\omega_{e}}{\omega_{b}} L_{m}\left(\Delta i_{q r}\right) \\
\frac{L_{s}}{\omega_{b}} p\left(\Delta i_{q s}\right)+\frac{L_{m}}{\omega_{b}} p\left(\Delta i_{q r}\right)=\left(\Delta v_{q s}\right)-R_{s}\left(\Delta i_{q s}\right)-\frac{\omega_{e}}{\omega_{b}} L_{s}\left(\Delta i_{d s}\right)-\frac{\omega_{e}}{\omega_{b}} L_{m}\left(\Delta i_{d r}\right) \\
\frac{L_{r}}{\omega_{b}} p\left(\Delta i_{d r}\right)+\frac{L_{m}}{\omega_{b}} p\left(\Delta i_{d s}\right)=\left(\Delta v_{d r}\right)+b_{1}\left(\Delta i_{q s}\right)-a_{1}\left(\Delta \omega_{r}\right)+c_{1}\left(\Delta i_{q r}\right)-R_{r}\left(\Delta i_{d r}\right)
\end{gathered}
$$

Where $a_{1}=\left(\frac{L_{m}}{\omega_{b}} i_{q s 0}+\frac{L_{r}}{\omega_{b}} i_{q r 0}\right) ; b_{1}=\frac{L_{m}}{\omega_{b}}\left(\omega_{e}-\omega_{r 0}\right) c_{1}=\frac{L_{r}}{\omega_{b}}\left(\omega_{e}-\omega_{r 0}\right)$

$$
\frac{L_{r}}{\omega_{b}} p\left(\Delta i_{q r}\right)+\frac{L_{m}}{\omega_{b}} p\left(\Delta i_{q s}\right)=\left(\Delta v_{q r}\right)-b_{1}\left(\Delta i_{d s}\right)+a_{2}\left(\Delta \omega_{r}\right)-c_{1}\left(\Delta i_{d r}\right)-R_{r}\left(\Delta i_{d r}\right)
$$

Where $a_{2}=\left(\frac{L_{m}}{\omega_{b}} i_{d s 0}+\frac{L_{r}}{\omega_{b}} i_{d r 0}\right)$

In the first step small signal analysis is carried out for the wind system without adding any controller. For this the other equations which contribute are the DC-link voltage and the PLL to synchronize the system to the grid.

Linearized equation of the DC-link model developed from (2.69) is:

$$
C p\left(\Delta v_{d c}\right)=a_{3}\left(\Delta v_{d c}\right)+\frac{1}{v_{d c 0}}\left(b_{2}-c_{2}\right)
$$

Where $a_{3}=\frac{1}{v_{d c 0}^{2}}\left(v_{d r 0} i_{d r 0}+v_{q r 0} i_{q r 0}-\left(v_{d g 0} i_{d g 0}+v_{q g 0} i_{q g 0}\right)\right)$;

$$
\begin{gathered}
b_{2}=\left(v_{d g 0}\left(\Delta i_{d g}\right)+i_{d g 0}\left(\Delta v_{d g}\right)+v_{q g 0}\left(\Delta i_{q g}\right)+i_{q g 0}\left(\Delta v_{q g}\right)\right) \\
c_{2}=\left(v_{d r 0}\left(\Delta i_{d r}\right)+i_{d r 0}\left(\Delta v_{d r}\right)+v_{q r 0}\left(\Delta i_{q r}\right)+i_{q r 0}\left(\Delta v_{q r}\right)\right)
\end{gathered}
$$

Linearized PLL equations are:

$$
p \Delta \theta_{p l l}=\Delta \omega_{p l l}
$$




$$
p\left(\Delta \omega_{p l l}\right)-k_{p p l l} p\left(\Delta v_{q s}\right)=k_{i p l l}\left(\Delta v_{q s}\right)
$$

The state space model is developed for the linearized model (4.8)- (4.16) in the form of:

$$
E(\Delta \dot{X})=A(\Delta X)
$$

The state variables without adding controller to the model are:

$$
\Delta X=\left[\begin{array}{llllllll}
\omega_{t} & \omega_{r} & i_{d s} & i_{q s} & i_{d r} & i_{q r} & v_{d c} & \theta_{p l l}
\end{array}\right]^{T}
$$

As described in [82], the real components of eigenvalues of $(E, A)$ gives the damping and the imaginary component gives the frequency of oscillations. For a complex pair of eigenvalues:

$$
\lambda=\sigma \pm j \omega
$$

Damping coefficients is given by $\xi=-\left(\frac{\sigma}{\sqrt{\sigma^{2}+\omega^{2}}}\right)$ and oscillating frequency is given by $f_{o s c}=(\omega / 2 \pi)$.

\section{Small signal model without controller:}

For the system without controller the eigenvalues of $(E, A)$ are plotted in the Matlab environment and the obtained eigenvalues of $(E, A)$ and damping coefficient and frequency oscillations are given in Table 4.1.

Table 4.1: Eigenvalues of $(E, A)$ of a grid connected system without controller.

\begin{tabular}{|c|c|c|c|c|c|}
\hline & $\lambda=\sigma \pm j \omega$ & $\sigma$ & $\omega$ & $\xi$ & $f_{\text {osc }}$ \\
\hline$\lambda_{1}, \lambda_{2}$ & $-18.13 \pm 507.09 i$ & -18.13 & 507.09 & 0.0357 & 80.7 \\
\hline$\lambda_{3}, \lambda_{4}$ & $-24.38 \pm 326.16 i$ & -24.38 & 326.16 & 0.0745 & 52 \\
\hline$\lambda_{5}, \lambda_{6}$ & $-0.51 \pm 0.70 i$ & -0.51 & 0.7 & 0.5889 & 0.1 \\
\hline$\lambda_{7}$ & -0.06 & -0.06 & 0 & 1 & 0 \\
\hline$\lambda_{8}$ & -10.0 & -10 & 0 & 1 & 0 \\
\hline
\end{tabular}


From Table 4.1, it can be observed that the eigenvalues of $(E, A)$ have negative real parts and this can be observed more easily from the Eigen plot shin in Figure 4.1. So from this a conclusion can be drawn that the system is small signal stable. There are a total of three complex pairs and one pair frequency is close to the fundamental frequency.

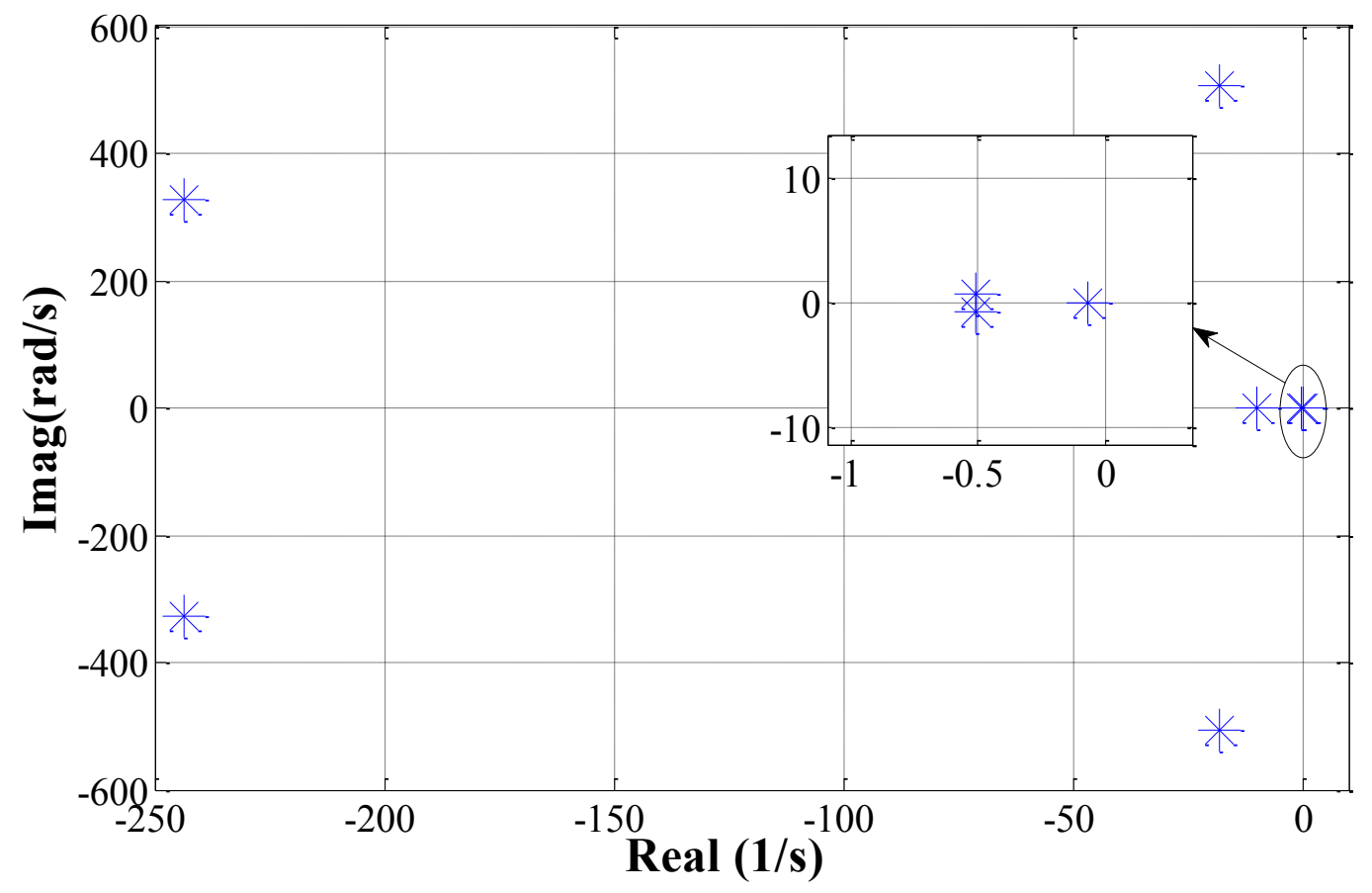

Figure 4.1: Eigen plot without controller

\section{Participation factors for the model without controller:}

The importance of implementing participation matrix for stability studies is described clearly in [82], which states that using the left and right Eigen vectors separately for finding relationship between states and modes is dependent on units and scaling associated with the state variables. So for this participation matrix is found to be the best solution which combines both right and left Eigen vectors. The general procedure for finding the participation matrix is as follows: In the first step the small signal model is developed and then from this model the eigenvalues of $(E, A)$ and eigenvectors are computed at an operating point. Then the right and 
left Eigen vectors are identified for the model. From this the participation matrix is defined as $P=\left[\begin{array}{llll}p_{1} & p_{2} & \ldots & p_{n}\end{array}\right]$ and $p_{i}=\left[\begin{array}{llll}p_{1 i} & p_{2 i} & \ldots & p_{n i}\end{array}\right]^{T}=\left[\begin{array}{llll}\phi_{1 i} \Psi_{i 2} & \phi_{2 i} \Psi_{i 2} & \ldots & \phi_{n i} \Psi_{i n}\end{array}\right]^{T}$ where $\phi_{1}$ represents the element of right eigenvector and $\Psi_{i}$ represent the left Eigen vector. From this the participation factor element can be derived and it is given as $p_{k i}=\phi_{k i} \Psi_{i k}[82]$. The participation matrix for the model without controller is obtained and is shown in Table 4.2:

Table 4.2: Participation matrix (without controller)

\begin{tabular}{|c|c|c|c|c|c|c|c|c|}
\hline & $\lambda_{1}$ & $\lambda_{2}$ & $\lambda_{3}$ & $\lambda_{4}$ & $\lambda_{5}$ & $\lambda_{6}$ & $\lambda_{7}$ & $\lambda_{8}$ \\
\hline$\omega_{t}$ & 0 & 0 & 0 & 0 & 0.1167 & 0.1167 & 0 & 0 \\
\hline$\omega_{r}$ & 0 & 0 & 0 & 0 & 0.5039 & 0.5039 & 0 & 0 \\
\hline$I_{d s}$ & 1.0637 & 1.0637 & 0.6209 & 0.6209 & 0 & 0 & 0 & 0 \\
\hline$I_{q s}$ & 0.9163 & 0.9163 & 1.3214 & 1.3214 & 0 & 0 & 0 & 0 \\
\hline$I_{d r}$ & 0.9016 & 0.9016 & 0.7618 & 0.7618 & 0 & 0 & 0 & 0 \\
\hline$I_{q r}$ & 0.9912 & 0.9912 & 1.3184 & 1.3184 & 0 & 0 & 0 & 0 \\
\hline$V_{d c}$ & 0 & 0 & 0 & 0 & 0 & 0 & 1.0 & 0 \\
\hline$\theta_{P L L}$ & 0 & 0 & 0 & 0 & 0 & 0 & 0 & 1.0 \\
\hline
\end{tabular}

From the participation matrix shown in Table 4.2, the following observations can be drawn:

- It can be observed that $\lambda_{1}$ and $\lambda_{2}$ are mainly associated with stator currents $i_{d s}$ and $i_{q s}$ The corresponding state variables for the obtained Eigen analysis is shown in Table 4.3

- Similarly $\lambda_{3}$ and $\lambda_{4}$ are mainly associated with the rotor currents $i_{d r}$ and $i_{q r}$ and it can be observed from Table 4.1 that the oscillating frequency is close to the fundamental frequency. 
- $\lambda_{5}$ and $\lambda_{6}$ are associated with the wind turbine speed and the generator speed and they oscillate at very low frequency.

- Finally $\lambda_{7}$ is associated with dc-link voltage $v_{d c}$ and $\lambda_{8}$ with $\theta_{p l l}$.

The eigenvalues of $(E, A)$ along with the corresponding states is given in Table 4.3

Table 4.3: Eigenvalues of $(E, A)$ and the corresponding state variables:

\begin{tabular}{|c|c|c|}
\hline & $\lambda=\sigma \pm \mathrm{j} \omega$ & State Variables $\Delta X$ \\
\hline$\lambda_{1}, \lambda_{2}$ & $-18.13 \pm 507.09 i$ & $I_{d s}$ and $I_{q s}$ \\
\hline$\lambda_{3}, \lambda_{4}$ & $-24.38 \pm 326.16 i$ & $I_{d r}$ and $I_{q r}$ \\
\hline$\lambda_{5}, \lambda_{6}$ & $-0.51 \pm 0.70 i$ & $\omega_{r}$ and $\omega_{t}$ \\
\hline$\lambda_{7}$ & -0.06 & $V_{d c}$ \\
\hline$\lambda_{8}$ & -10.0 & $\theta_{P L L}$ \\
\hline
\end{tabular}

\section{Small signal model with controller:}

The above discussed equations are same to the system equipped with the controller.

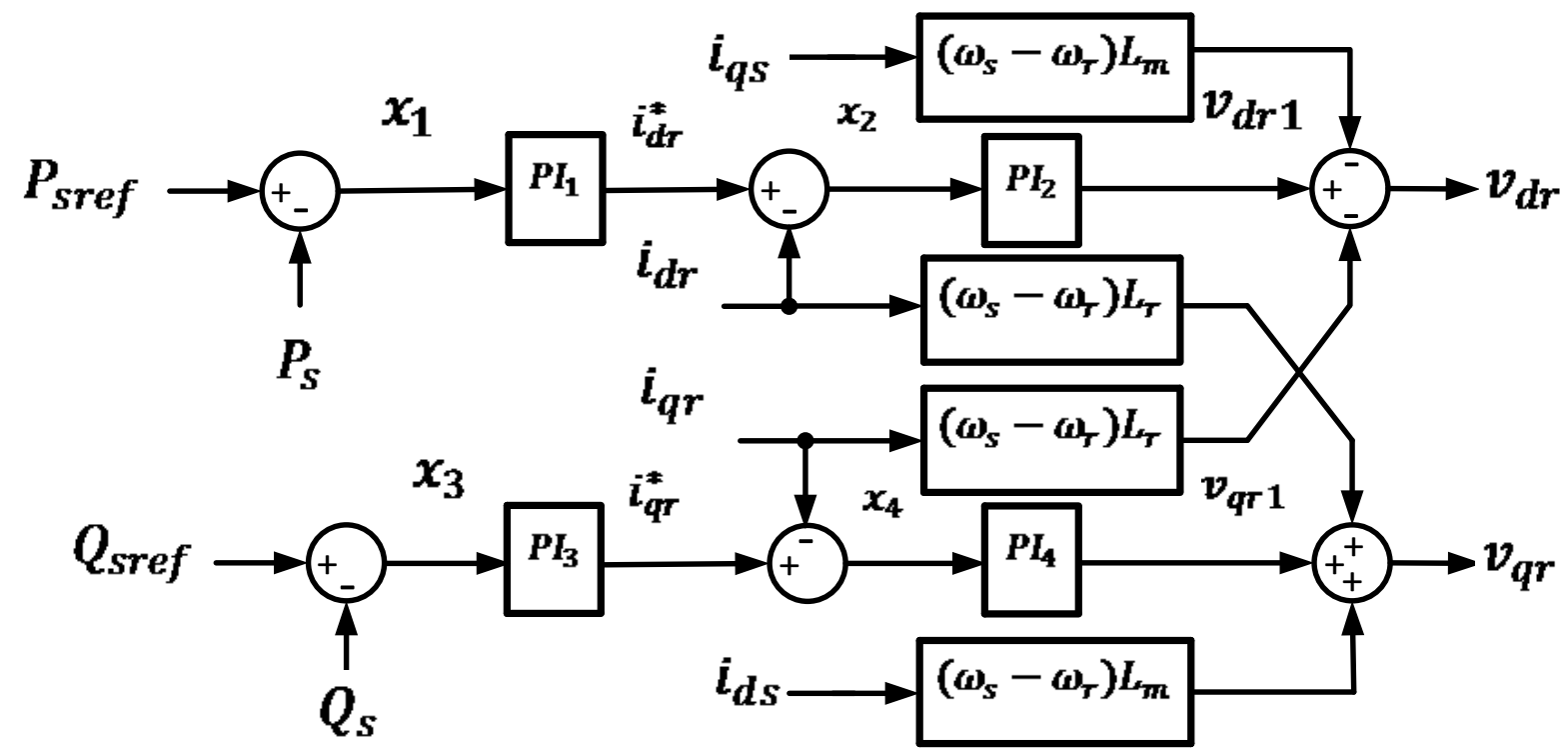

Figure 4.2: Rotor side controller (RSC) 
Additional rotor side controller and grid side controller equations are added to the system. For easy modeling purpose the RSC figure is repeated again. The mathematical modeling for controllers is given in Chapter 3, in this section the expanded large signal equations are derived and further the linearized model is developed for these equations. Figure 4.2, shows the rotor side controller and Figure 4.3, shows the design of grid side controller.

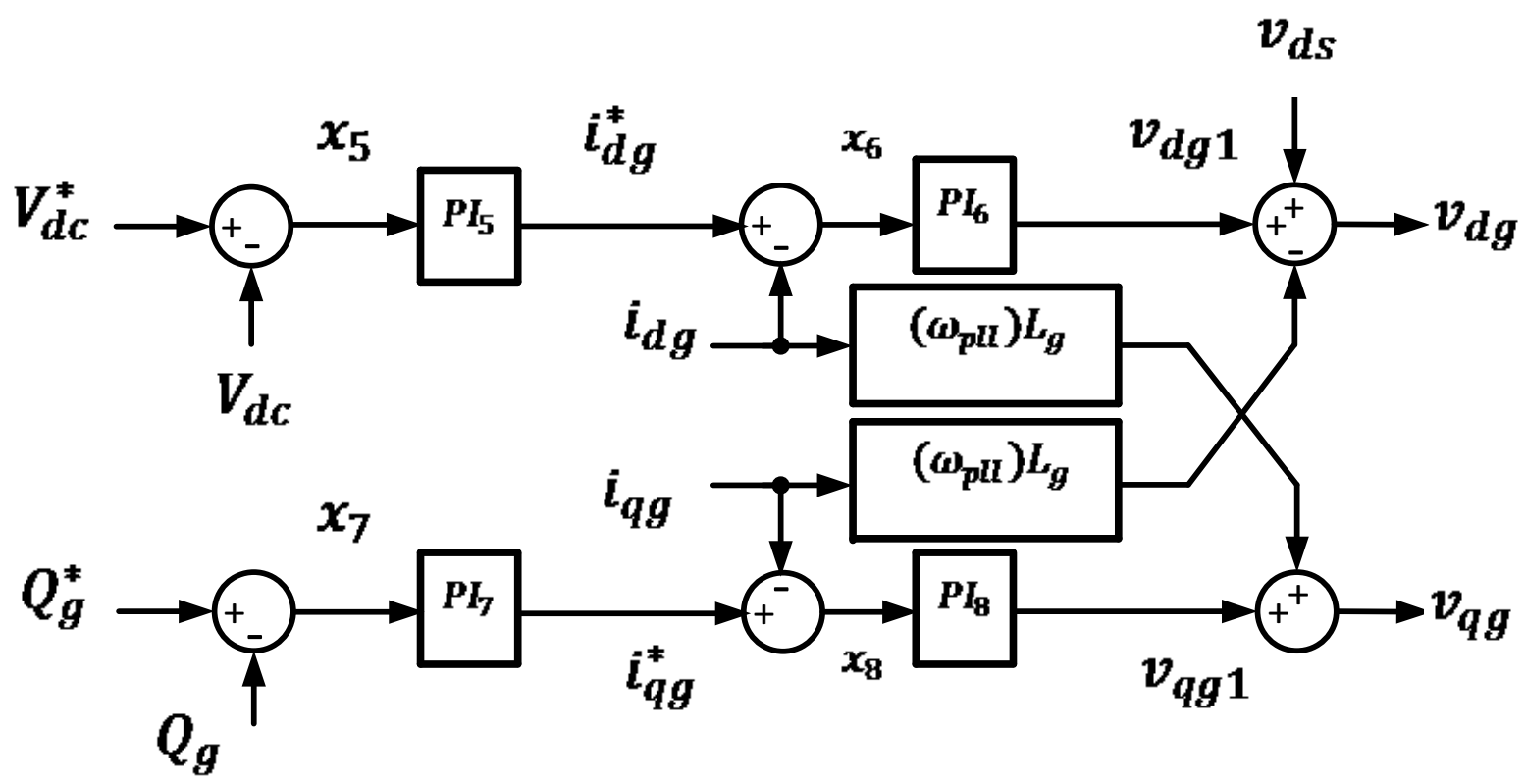

Figure 4.3: Grid side controller (GSC)

From Figure 4.2 and 4.3; the large signal model for the controllers can be derived as:

Rotor side controller model:

$$
\begin{gathered}
p x_{1}=P_{\text {sref }}-P_{s} \\
p x_{2}=K_{p 1}\left(P_{\text {sref }}-P_{s}\right)+K_{i 1} x_{1}-i_{d r} \\
v_{d r}=K_{p 2} K_{p 1}\left(P_{\text {sref }}-P_{s}\right)+K_{p 2} K_{i 1} x_{1}-K_{p 2} i_{d r}+K_{i 2} x_{2}-\left(\omega_{s}-\omega_{r}\right) L_{r} i_{q r} \\
-\left(\omega_{s}-\omega_{r}\right) L_{m} i_{q s} \\
p x_{3}=Q_{s r e f}-Q_{s} \\
p x_{4}=K_{p 3}\left(Q_{\text {sref }}-Q_{s}\right)+K_{i 3} x_{3}-i_{q r}
\end{gathered}
$$




$$
\begin{gathered}
v_{q r}=K_{p 4} K_{p 3}\left(Q_{s r e f}-Q_{s}\right)+K_{p 4} K_{i 3} x_{3}-K_{p 4} i_{q r}+K_{i 4} x_{4}+\left(\omega_{s}-\omega_{r}\right) L_{r} i_{d r} \\
-\left(\omega_{s}-\omega_{r}\right) L_{m} i_{d s}
\end{gathered}
$$

Grid side controller model:

$$
\begin{gathered}
p x_{5}=V_{d c}^{*}-V_{d c} \\
p x_{6}=K_{p 5}\left(V_{d c}^{*}-V_{d c}\right)+K_{i 5} x_{5}-i_{d g} \\
v_{d g}=K_{p 6} K_{p 5}\left(V_{d c}^{*}-V_{d c}\right)+K_{p 6} K_{i 5} x_{5}-K_{p 6} i_{d g}+K_{i 6} x_{6}+L_{g}\left(\omega_{p l l}\right) i_{q g}+v_{d s} \\
p x_{7}=Q_{g}^{*}-Q_{g} \\
p x_{8}=K_{p 7}\left(Q_{g}^{*}-Q_{g}\right)+K_{i 7} x_{7}-i_{q g} \\
v_{q g}=K_{p 8} K_{p 7}\left(Q_{g}^{*}-Q_{g}\right)+K_{p 8} K_{i 7} x_{7}-K_{p 8} i_{q g}+K_{i 8} x_{8}-L_{g}\left(\omega_{p l l}\right) i_{d g}
\end{gathered}
$$

Linearized model of the rotor side controller:

$$
\begin{gathered}
p\left(\Delta x_{1}\right)=-\left(v_{d s 0}\left(\Delta i_{d s}\right)+i_{d s 0}\left(\Delta v_{d s}\right)+v_{q s 0}\left(\Delta i_{q s}\right)+i_{q s 0}\left(\Delta v_{q s}\right)\right) \\
p\left(\Delta x_{2}\right)=-K_{p 1}\left(v_{d s 0}\left(\Delta i_{d s}\right)+i_{d s 0}\left(\Delta v_{d s}\right)+v_{q s 0}\left(\Delta i_{q s}\right)+i_{q s 0}\left(\Delta v_{q s}\right)\right)+K_{i 1} \Delta x_{1} \\
-\Delta i_{d r} \\
\Delta v_{d r}=-K_{p 2} K_{p 1}\left(v_{d s 0}\left(\Delta i_{d s}\right)+i_{d s 0}\left(\Delta v_{d s}\right)+v_{q s 0}\left(\Delta i_{q s}\right)+i_{q s 0}\left(\Delta v_{q s}\right)\right) \\
+\left(L_{m}\left(\omega_{r 0}-\omega_{s}\right)-K_{p 2} K_{p 1} v_{q s 0}\right)\left(\Delta i_{q s}\right)+K_{p 2} K_{i 1} \Delta x_{1}-K_{p 2} \Delta i_{d r}+K_{i 2} \Delta x_{2} \\
-\left(\omega_{r 0}-\omega_{s}\right) L_{r} \Delta i_{q r}+L_{r}\left(i_{q r o}+i_{q s o}\right) \Delta \omega_{r} \\
p\left(\Delta x_{4}\right)=K_{p 3}\left(-v_{d s 0}\left(\Delta i_{q s}\right)-i_{q s 0}\left(\Delta v_{d s}\right)+v_{q s 0}\left(\Delta i_{d s}\right)+i_{d s 0}\left(\Delta v_{q s}\right)\right)+K_{i 1} \Delta x_{3} \\
-\Delta i_{q r}
\end{gathered}
$$




$$
\begin{aligned}
\Delta v_{q r} & =K_{p 4} K_{p 3}\left(-v_{d s 0}\left(\Delta i_{q s}\right)-i_{q s 0}\left(\Delta v_{d s}\right)+v_{q s 0}\left(\Delta i_{d s}\right)+i_{d s 0}\left(\Delta v_{q s}\right)\right) \\
& +\left(L_{m}\left(\omega_{s}-\omega_{r 0}\right)+K_{p 4} K_{p 3} v_{q s 0}\right)\left(\Delta i_{d s}\right)+K_{p 4} K_{i 3} \Delta x_{3}-K_{p 4} \Delta i_{q r}+K_{i 4} \Delta x_{4} \\
& +\left(\omega_{s}-\omega_{r 0}\right) L_{r} \Delta i_{d r}-L_{r}\left(i_{d r o}+i_{d s o}\right) \Delta \omega_{r}
\end{aligned}
$$

Linearized model of the grid side controller:

$$
\begin{gathered}
p \Delta x_{5}=-\Delta V_{d c} \\
p \Delta x_{6}=-K_{p 5}\left(\Delta V_{d c}\right)+K_{i 5} \Delta x_{5}-\Delta i_{d g} \\
\Delta v_{d g}=-K_{p 6} K_{p 5}\left(\Delta V_{d c}\right)+K_{p 6} K_{i 5} \Delta x_{5}-K_{p 6} \Delta i_{d g}+K_{i 6} \Delta x_{6}+L_{g}\left(\omega_{p l l 0}\right) \Delta i_{q g}+\Delta v_{d s} \\
+L_{g} i_{q g 0}\left(\Delta \omega_{p l l}\right)(4.28) \\
p \Delta x_{7}=-\Delta Q_{g} \\
\begin{array}{l}
p \Delta x_{8 g}=-K_{p 7}\left(\Delta Q_{g}\right)+K_{i 7} \Delta x_{7}-\Delta i_{q g} \\
\left(-K_{p 8} K_{p 7} v_{d s 0}-K_{p 8}\right)\left(\Delta i_{q g}\right)-K_{p 8} K_{p 7} i_{q g 0}\left(\Delta v_{d s}\right)+\left(K_{p 8} K_{p 7} v_{q s 0}-L_{g} \omega_{p l l 0}\right)\left(\Delta i_{d g}\right) \\
+K_{p 8} K_{p 7} i_{d g 0}\left(\Delta v_{q s}\right)+K_{p 8} K_{i 7} \Delta x_{7}-K_{p 8} i_{q g}+K_{i 8} \Delta x_{8} \\
-L_{g} i_{d g 0}\left(\Delta \omega_{p l l}\right)
\end{array}
\end{gathered}
$$

Where $\Delta Q_{g}=v_{d s 0} \Delta i_{q g}+i_{q g 0} \Delta v_{d s}-\left(v_{q s 0} \Delta i_{d g}+i_{d g 0} \Delta v_{q s}\right)$

Linearized grid equations:

$$
\begin{aligned}
& \operatorname{Lp} \Delta\left(i_{d s}+i_{d g}\right) \\
& =\Delta v_{d s}-r \Delta i_{d s}-r \Delta i_{d g}+\omega_{0} L \Delta i_{q s}+\omega_{0} L \Delta i_{q g}+L\left(i_{q s 0}+i_{q g 0}\right)\left(\Delta \omega_{p l l}\right) \\
& -E \sin \delta_{0}\left(\Delta \delta_{p l l}\right) \\
& \operatorname{Lp} \Delta\left(i_{q s}+i_{q g}\right) \\
& =\Delta v_{q s}-r \Delta i_{q s}-r \Delta i_{q g}-\omega_{0} L \Delta i_{d s}-\omega_{0} L \Delta i_{d g}-L\left(i_{d s 0}+i_{d g 0}\right)\left(\Delta \omega_{p l l}\right) \\
& -E \cos \delta_{0}\left(\Delta \delta_{p l l}\right)
\end{aligned}
$$




$$
\begin{aligned}
& L_{g} p\left(\Delta i_{d g}\right)=\left(\Delta v_{d g}\right)-R_{g}\left(\Delta i_{d g}\right)+\omega_{0} L_{g}\left(\Delta i_{q g}\right)+L_{g} i_{q g 0}\left(\Delta \omega_{p l l}\right)-\Delta v_{d s} \\
& L_{g} p\left(\Delta i_{q g}\right)=\left(\Delta v_{q g}\right)-R_{g}\left(\Delta i_{q g}\right)-\omega_{0} L_{g}\left(\Delta i_{d g}\right)-L_{g} i_{d g 0}\left(\Delta \omega_{p l l}\right)-\Delta v_{q s}
\end{aligned}
$$

With this all the linearized equations for a DFIG based wind generation system are derived. Equations (4.32)- (4.46) contribute the linearized model of the controllers and the grid, similarly equations (4.8)- (4.17) contribute the mechanical and generator linearized model along with PLL. With these models, small signal analysis can be done for the entire wind model with the controllers. The obtained eigenvalues of $(E, A)$ are shown in Table 4.4:

Table 4.4: Eigenvalues of $(E, A)$ for grid integrated DFIG model (with controller)

\begin{tabular}{|c|c|c|c|c|c|}
\hline & $\lambda=\sigma \pm j \omega$ & $\sigma$ & $\omega$ & $\xi$ & $f_{\text {osc }}$ \\
\hline$\lambda_{1}, \lambda_{2}$ & $-749.98 \pm 899.7 i$ & -749.98 & 899.7 & 0.6403 & 143.1 \\
\hline$\lambda_{3}$ & -702.98 & -702.98 & 0 & 1 & 0 \\
\hline$\lambda_{4}, \lambda_{5}$ & $-3.61 \pm 367.32 i$ & -3.61 & 367.32 & 0.0098 & 58.46 \\
\hline$\lambda_{6}, \lambda_{7}$ & $-55.78 \pm 355.88 i$ & -55.78 & 355.88 & 0.1548 & 56.6 \\
\hline$\lambda_{8}, \lambda_{9}$ & $-53.47 \pm 38.37 i$ & -53.47 & 38.37 & 0.8125 & 6.1 \\
\hline$\lambda_{10}$ & -40.5 & -40.5 & 0 & 1 & 0 \\
\hline$\lambda_{11}$ & -33.8 & -33.8 & 0 & 1 & 0 \\
\hline$\lambda_{12}, \lambda_{13}$ & $-8.95 \pm 8.47 i$ & -8.95 & 8.47 & 0.7263 & 1.35 \\
\hline$\lambda_{14}$ & -0.07 & -0.07 & 0 & 1 & 0 \\
\hline$\lambda_{15}, \lambda_{16}$ & $-0.51 \pm 0.7 i$ & -0.51 & 0.7 & 0.5889 & 0.1114 \\
\hline$\lambda_{17}, \lambda_{18}$ & $-6.09 \pm 0.16 i$ & -6.09 & 0.16 & 0.9 & 0.026 \\
\hline$\lambda_{19}$ & -0.9304 & -0.9304 & 0 & 1 & 0 \\
\hline$\lambda_{20}$ & -0.93012 & -0.93012 & 0 & 1 & 0 \\
\hline
\end{tabular}


From Table 4.4 and Figure 4.4 it can be observed that all the real parts of the eigenvalues of $(E, A)$ are negative. So the system is small signal stable.

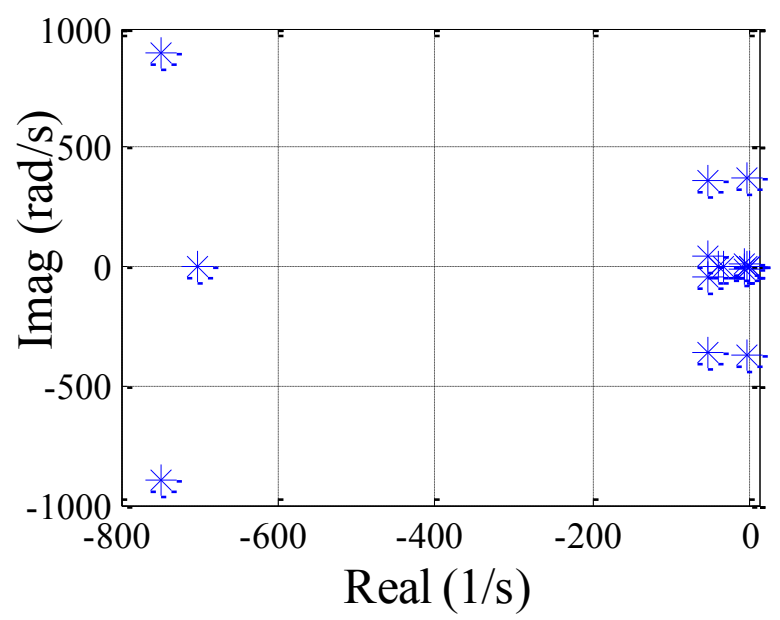

Figure 4.4: Eigen plot with controller

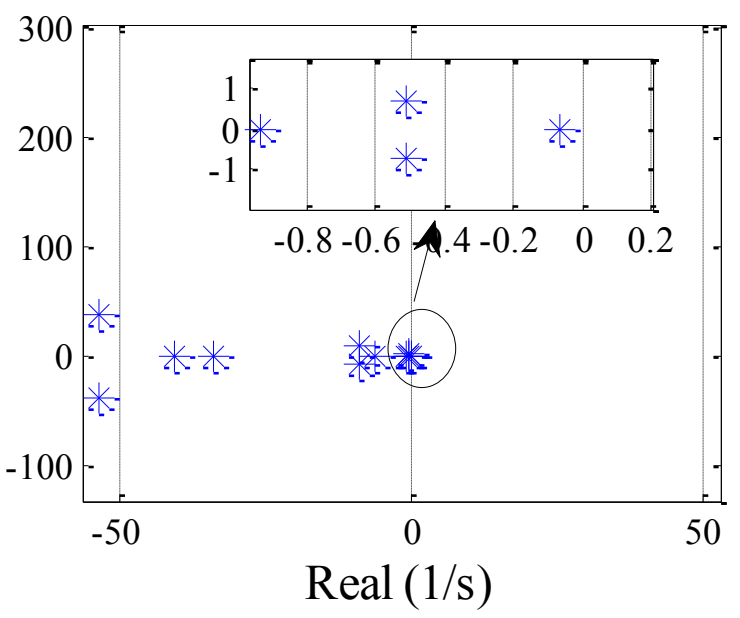

Figure 4.5: Zoomed Eigen plot with controller

The Figure 4.5 shows a zoomed plot for the eigenvalues of $(E, A)$ obtained with the controller and it can be observed that none of the values are on the right hand side of the imaginary axis. So finally the system fulfilled the stability rule according to the Lyapunov's first method.

When the system is shown with the controller the state variables are:

The state matrix is given by $\Delta X=$

$\left[\begin{array}{lllllllllllllllllllll}\omega_{t} & \theta_{t w} & \omega_{r} & i_{d s} & i_{q s} & i_{d r} & i_{q r} & v_{q s} & v_{d c} & i_{d g} & i_{q g} & \theta_{p l l} & x_{1} & x_{2} & x_{3} & x_{4} & x_{4} & x_{5} & x_{6} & x_{7} & x_{8}\end{array}\right]^{T}$

The participation matrix for the model is as given in Table 4.5.

From the participation matrix the following conclusion can be made:

- The wind turbine rotor speed state is associated with eigenvalue $\lambda_{14}$ and from Table 4.4 it can be observed that its frequency is zero and the value is very close to the imaginary axis, $\omega_{t}$ is one of the main state which is effecting the stability of the system.

- Generator speed $\omega_{r}$ and shaft twist angle $\theta_{t w}$ are associated with eigenvalues $\lambda_{15}$ and $\lambda_{16}$ and their oscillating frequencies and the damping factors are shown in Table 4.4 
Table 4.5: participation factors with controllers.

\begin{tabular}{|l|l|l|l|l|l|l|l|l|l|l|l|l|l|}
\hline & $\lambda_{1,2}$ & $\lambda_{3}$ & $\lambda_{4,5}$ & $\lambda_{6,7}$ & $\lambda_{8,9}$ & $\lambda_{10}$ & $\lambda_{11}$ & $\lambda_{12,13}$ & $\lambda_{14}$ & $\lambda_{15,16}$ & $\lambda_{17,18}$ & $\lambda_{19}$ & $\lambda_{20}$ \\
\hline$\omega_{t}$ & 0 & 0 & 0 & 0 & 0 & 0 & 0 & 0 & .8 & 0.1 & 0 & 0 & 0 \\
\hline$\theta_{t w}$ & 0 & 0 & 0 & 0 & 0 & 0 & 0 & 0 & .0 & 0.6 & 0 & 0 & 0 \\
\hline$\omega_{r}$ & 0 & 0 & 0 & 0 & 0 & 0 & 0 & 0 & .1 & 0.5 & 0 & 0 & 0 \\
\hline$I_{d s}$ & .07 & 1.1 & .06 & .058 & .15 & .007 & .001 & .012 & 0 & 0 & 0 & 0 & 0 \\
\hline$I_{q s}$ & .09 & .12 & .04 & 0.49 & 0.01 & .004 & .005 & .002 & 0 & 0 & 0 & 0 & 0 \\
\hline$I_{d r}$ & .01 & .05 & .4 & 0.06 & 0.08 & .007 & .002 & .005 & 0 & 0 & 0 & 0 & 0 \\
\hline$I_{q r}$ & .03 & .04 & .56 & 0.08 & 0.00 & .004 & .005 & .001 & 0 & 0 & 0 & 0 & 0 \\
\hline$V_{q S}$ & .09 & .05 & .01 & .173 & 0.04 & .001 & .003 & 0.74 & 0 & 0 & .011 & 0 & 0 \\
\hline$V_{d c}$ & .05 & .04 & .14 & .112 & 0.89 & .025 & 0.01 & .018 & 0 & 0 & .006 & 0 & 0 \\
\hline$I_{d g}$ & .32 & 0 & .03 & .147 & .064 & .002 & 0 & .024 & 0 & 0 & 0.0 & 0 & 0 \\
\hline$I_{q g}$ & .41 & 0 & .08 & 0.13 & .036 & 0 & 0 & .013 & 0 & 0 & 0 & 0 & 0 \\
\hline$\theta_{P L L}$ & 0 & 0 & 0 & .107 & 0.06 & .001 & .002 & 0.54 & 0 & 0 & .007 & 0 & 0 \\
\hline$X_{1}$ & 0 & 0 & 0 & .010 & .003 & .508 & 0.51 & .002 & 0 & 0 & 0 & 0 & 0 \\
\hline$X_{2}$ & 0 & 0 & 0 & 0 & 0 & 0 & 0 & 0 & 0 & 0 & 0 & .02 & 1 \\
\hline$X_{3}$ & 0 & 0 & 0 & .009 & .048 & 0.5 & 0.5 & 0.01 & 0 & 0 & 0 & 0 & 0 \\
\hline$X_{4}$ & 0 & 0 & 0 & 0 & 0 & 0 & 0 & 0 & 0 & 0 & 0 & 1 & 0 \\
\hline$X_{5}$ & 0 & 0 & .02 & .015 & .883 & .064 & .011 & .027 & 0 & 0 & .017 & 0 & 0 \\
\hline$X_{6}$ & 0 & 0 & 0 & 0 & .051 & .004 & 0 & .021 & 0 & 0 & .524 & 0 & 0 \\
\hline$X_{7}$ & .14 & .08 & .01 & .276 & .004 & .016 & .00 & .118 & 0 & 0 & .014 & 0 & 0 \\
\hline$X_{8}$ & 0 & 0 & 0 & .006 & .001 & .001 & 0 & .061 & 0 & 0 & .501 & 0 & 0 \\
\hline
\end{tabular}


- $x_{2}$ and $x_{4}$ are associated with $\lambda_{20}$ and $\lambda_{19}$ respectively and they are very close to imaginary axis.

These states and their corresponding parameters along with the controllers need to be designed with care as they effect the stability of the system. In this thesis work the power system stabilizer is implemented at the inputs of $x_{2}$ and $x_{4}$ states and obtained better performance which is discussed in Chapter 5.

Table 4.6: State variables corresponding to eigenvalues of $(E, A)$

\begin{tabular}{|c|c|c|}
\hline & $\lambda=\sigma \pm \mathrm{j} \omega$ & State Variables $\Delta X$ \\
\hline$\lambda_{1}, \lambda_{2}$ & $-749.98 \pm 899.7 i$ & $i_{d g}$ and $i_{q g}$ \\
\hline$\lambda_{3}$ & -702.98 & $i_{d s}$ \\
\hline$\lambda_{4}, \lambda_{5}$ and $i_{q r}$ \\
\hline$\lambda_{6}, \lambda_{7}$ & $-3.61 \pm 367.32 i$ & $i_{q s}$ and $x_{7}$ \\
\hline$\lambda_{8}, \lambda_{9}$ & $-55.78 \pm 355.88 i$ & $V_{d c}$ and $x_{5}$ \\
\hline$\lambda_{10}$ & $-53.47 \pm 38.37 i$ & $x_{3}$ \\
\hline$\lambda_{11}$ & -40.5 & $x_{1}$ \\
\hline$\lambda_{12}, \lambda_{13}$ & -33.8 & $v_{q s}$ and $\theta_{p l l}$ \\
\hline$\lambda_{14}$ & $-8.95 \pm 8.47 i$ & $\omega_{t}$ \\
\hline$\lambda_{15}, \lambda_{16}$ & -0.07 & $\omega_{r}$ and $\theta_{t w}$ \\
\hline$\lambda_{17}, \lambda_{18}$ & $-0.51 \pm 0.7 i$ & $x_{6}$ and $x_{8}$ \\
\hline$\lambda_{19}$ & $-6.09 \pm 0.16 i$ & $x_{4}$ \\
\hline$\lambda_{20}$ & -0.9304 & $x_{2}$ \\
\hline & -0.93012 & \\
\hline & & \\
\hline & & \\
\hline
\end{tabular}


The other eigenvalues of $(E, A)$ and the states associated with them are shown in Table 4.6.

\subsection{Large Signal Analysis of a DFIG based Wind Generation System:}

In large signal analysis the mathematical model developed in Chapter 2 and the control blocks described in Chapter 3 are implemented in Simulink environment. In Section 4.2, the small signal model discussed is a fully mathematical model and no simulations are shown. Moreover in small signal stability analysis it is difficult to conduct analysis during fault conditions and observe the behaviour of the system. In large signal analysis the simulations are carried out during fault conditions and the system behaviour is observed.

In this section two models are analyzed, first analysis is carried out for a detailed switching model with the power electronic converters, this is termed as the detailed model. In the next step the power electronic converters are replaced by voltage sources and this model is termed as the large signal average model. The average model is mainly used for validating the detailed large signal model. The mathematical model developed for small signal analysis is based on the averaged large signal model. As the averaged large signal model is small signal stable the same averaged large signal model is used for validating the developed large signal detailed model. Finally system response is observed by applying a single line to ground fault at the grid end of the system.

The main aspects that need to be discussed for performing large signal analysis are the reference commands that are given to the controllers and the modes of control. The two control strategies are mentioned in Chapter 3 and the design is shown in Section 4.2. In small signal analysis the reference commands are set at constant values as the system analysis is performed at an operating point, so there is no much attention on the reference commands in small signal analysis. Whereas in large signal analysis the system is dynamic and some of the reference 
commands tend to change along with the operating conditions. The different reference commands which are used for control of the model are the stator active and reactive power references used at the RSC, i.e. $P_{S}^{*}$ and $Q_{S}^{*}$ respectively. Whereas at the GSC the reference commands are the dc-link voltage reference and grid reactive power reference. In these commands $P_{S}^{*}$ is the only reference which is related to the speed and all the other reference commands are set as constant values.

The simulation is run for a time period of $\tau=40 \mathrm{~s}$. The obtained simulations results are discussed below.

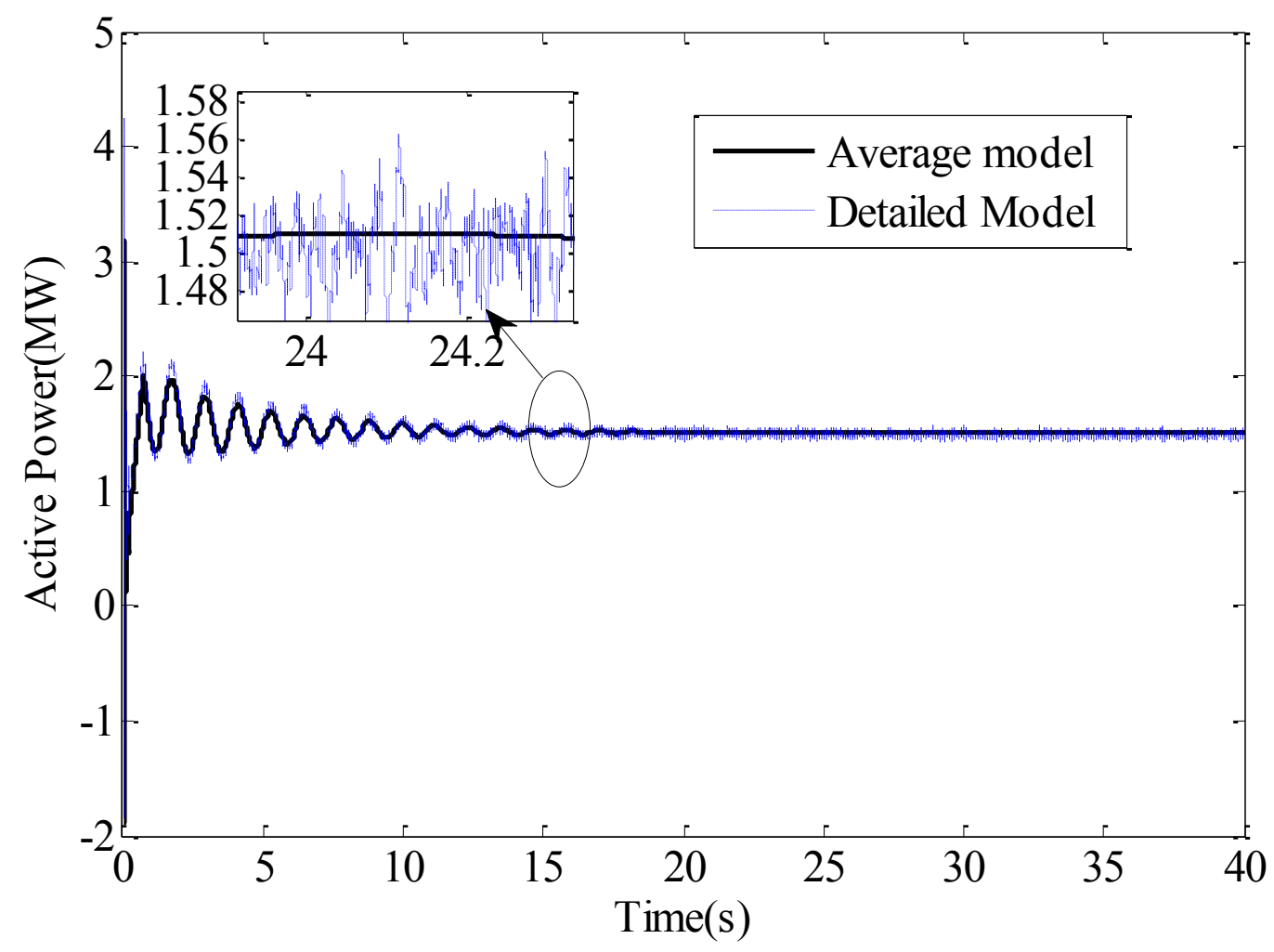

Figure 4.6: Plot for active power

Figure 4.6, describe the characteristics of active power supplied to the grid. It shows both the detailed switched model and the averaged model active power. Reactive power plot and generator speed plot are shown in Figure 4.7 and 4.8: 


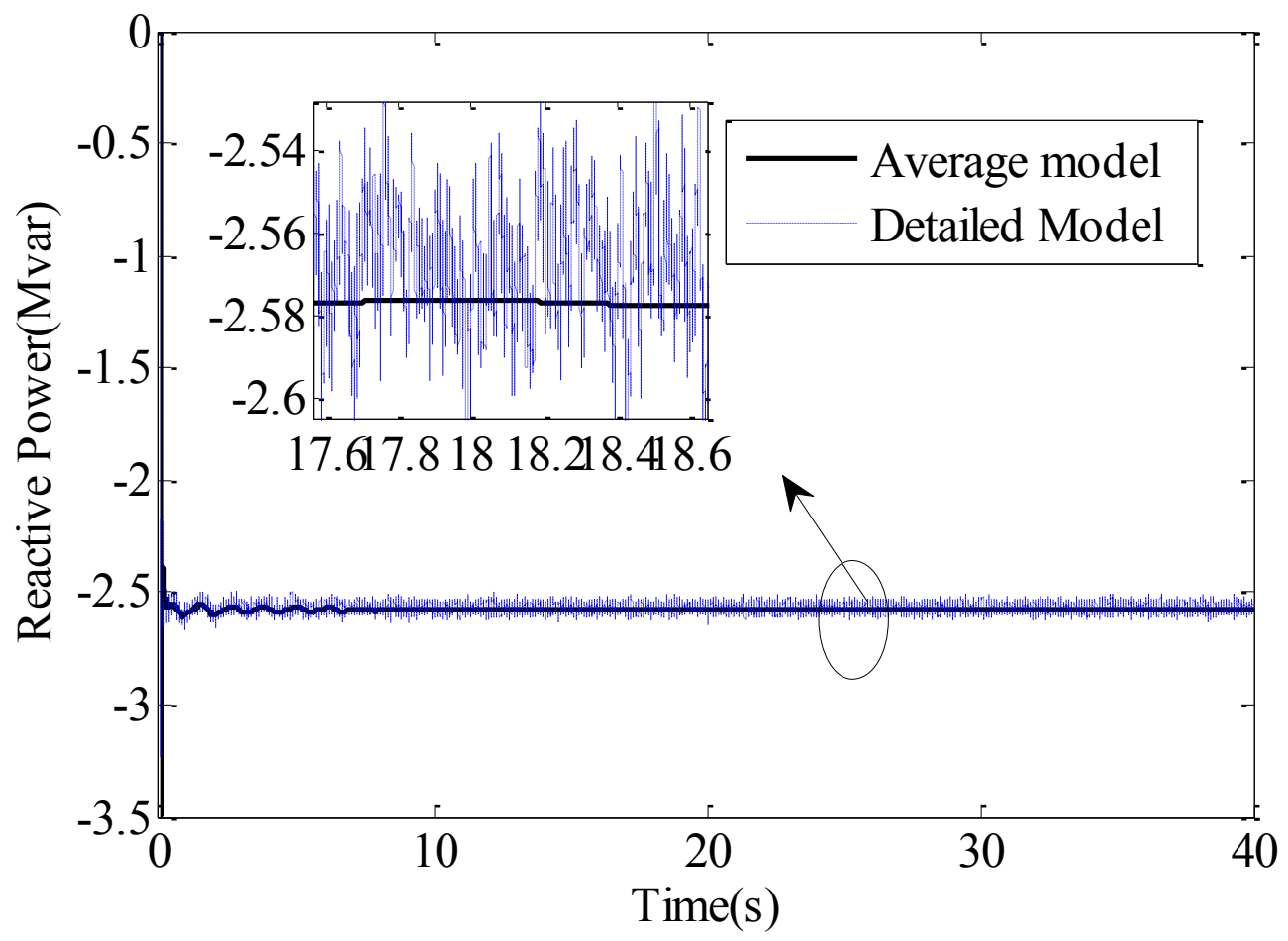

Figure 4.7: Reactive power

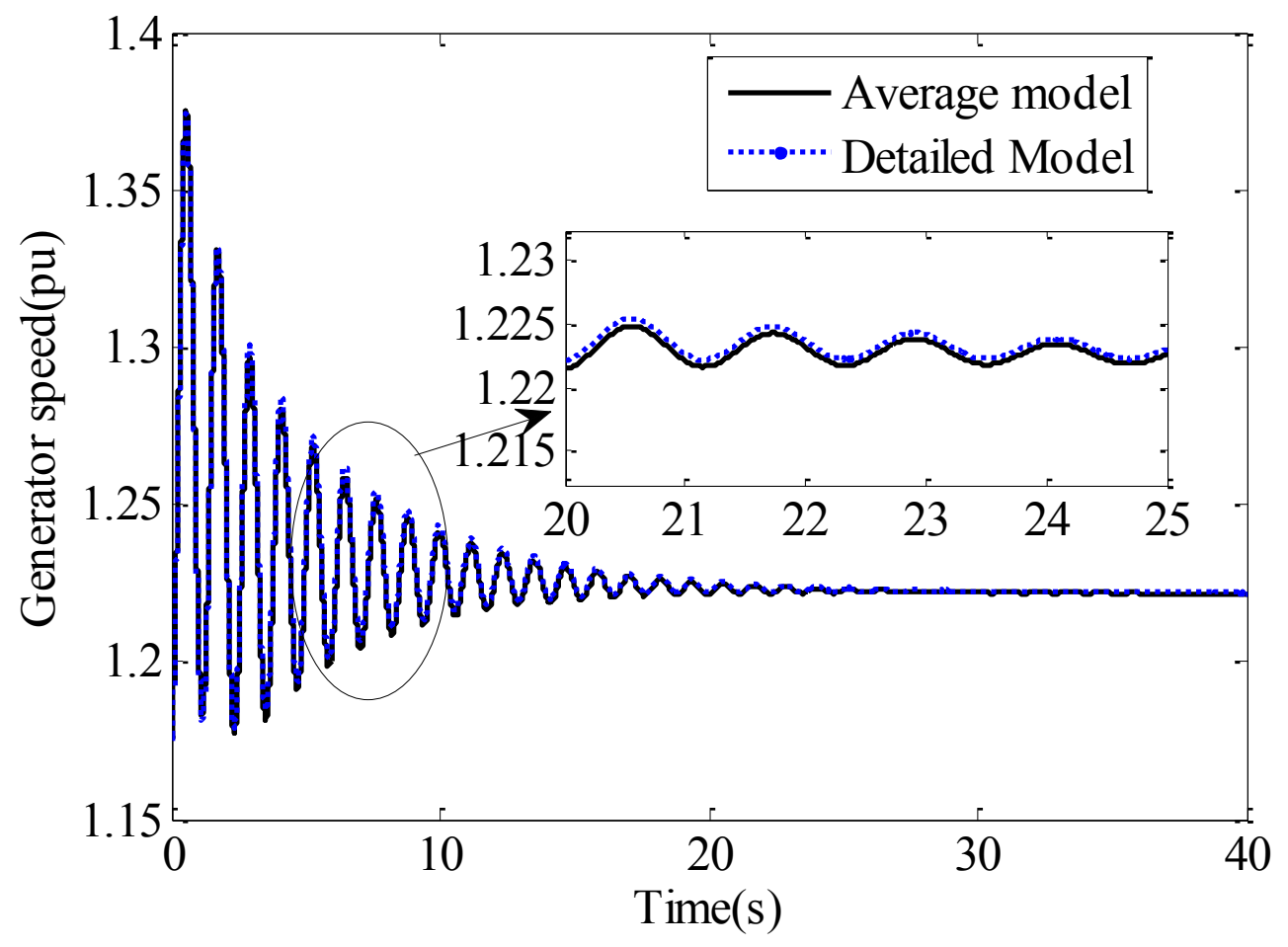

Figure 4.8: Generator speed 
In the above figures the averaged and detailed models are plotted and from the figures it can be observed that the waveforms are similar for both the models. Finally the plot for DC-link is shown in Figure 4.9.

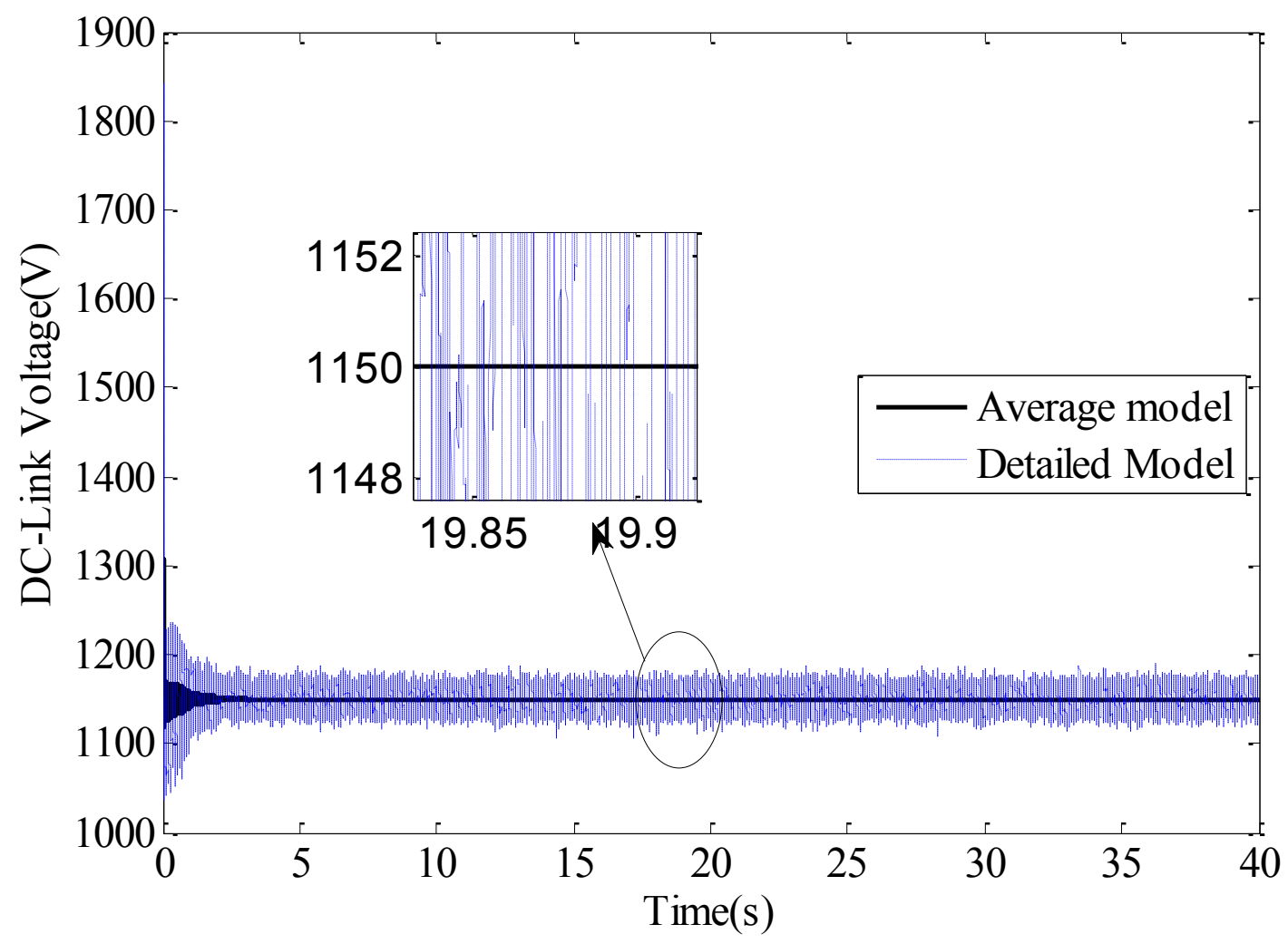

Figure 4.9: DC-link plot.

The parameters for the model developed is given in appendix B. The model operated at a specific operating conditions with the wind speed equal to $15 \mathrm{~m} / \mathrm{s}$. The machine is operated at above synchronous speed, the power is supplied to the grid from the rotor side converter. The reactive reference powers are kept at zero and dc-link reference is the nominal dc link voltage. All the plots shown above are drawn when the system is operating under the normal and rated operating conditions. Further analysis can be carried out for different operating condition of wind speed and when the machine is operating at sub-synchronous mode of operation, these are not shown in this research work as the main focus of this work is on stability studies and 
design of power system stabilizer for wind system. However for further analysis on the system response to the fault conditions, a three phase to ground fault is applied at the grid end that is at the point of common connection where the grid and the wind system are connected. System response to three phase to ground fault. The model is run for a time period of $\tau=40 \mathrm{~s}$ and the fault is applied for a period of $\tau=30 s$ to $\tau=32 s$.

The obtained results are:

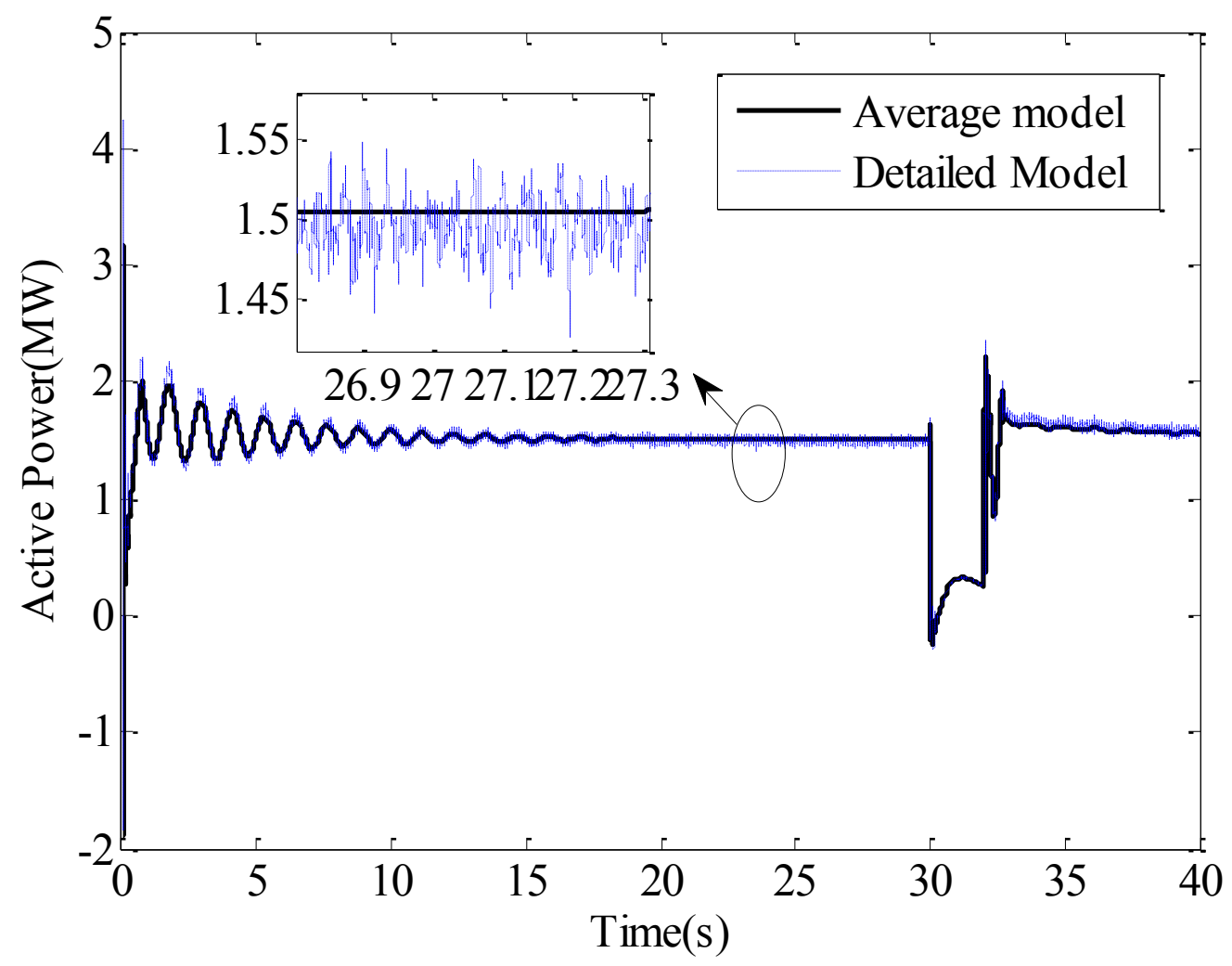

Figure 4.10: Active power during three phase fault at the grid end.

As discussed above the fault is applied for time period of $2 s$ and the active power obtained is shown in Figure 4.10. The fault is applied for both the detailed and averaged mode and the obtained waveforms are similar in both the cases. Due to the fault applied a dip in the power curve can be observed in Figure 4.10. From the Figure 4.10 it can be concluded that the power which is derived from stator voltages and the total current supplied to the grid causes dip in 
the stator voltages thus effecting the machine operation. In the similar way the plots are shown for the reactive power and the DC-link in Figure 4.11 and 4.12 respectively.

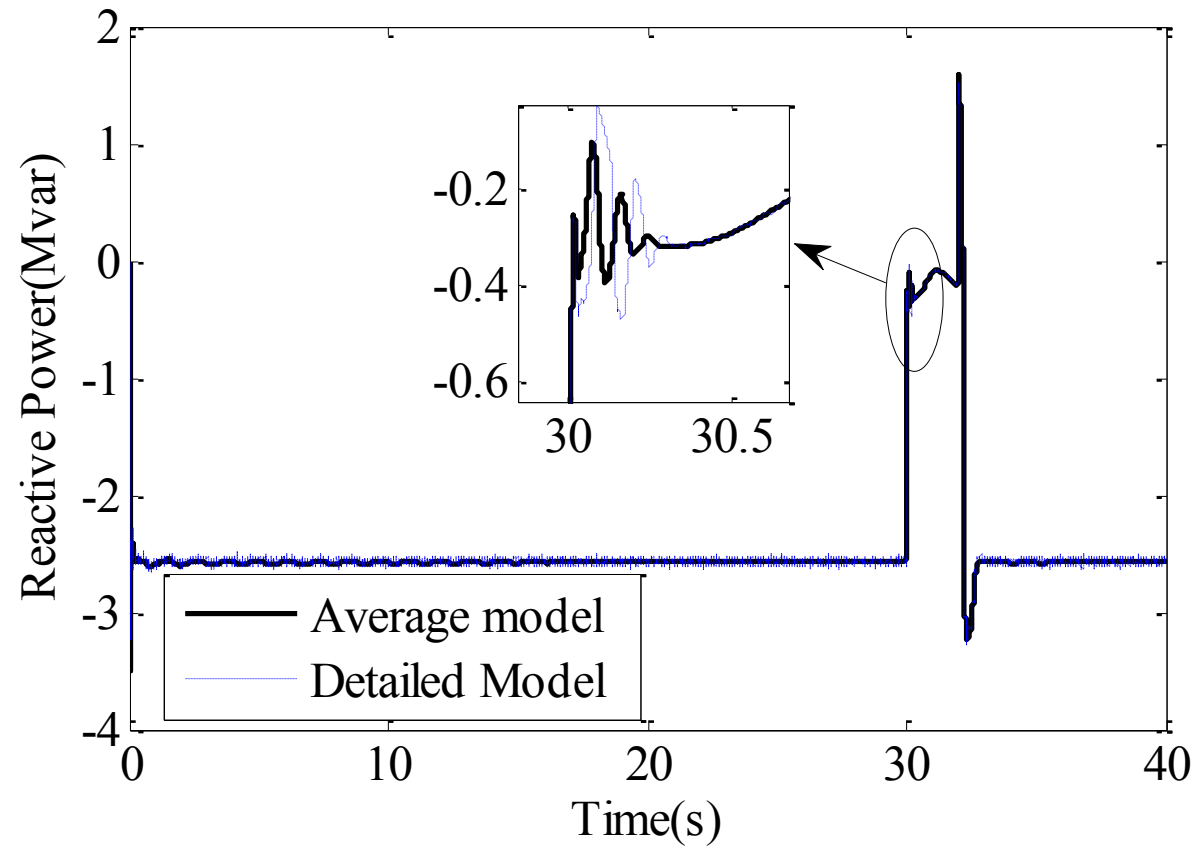

Figure 4.11: Reactive power during three phase fault at the grid end.

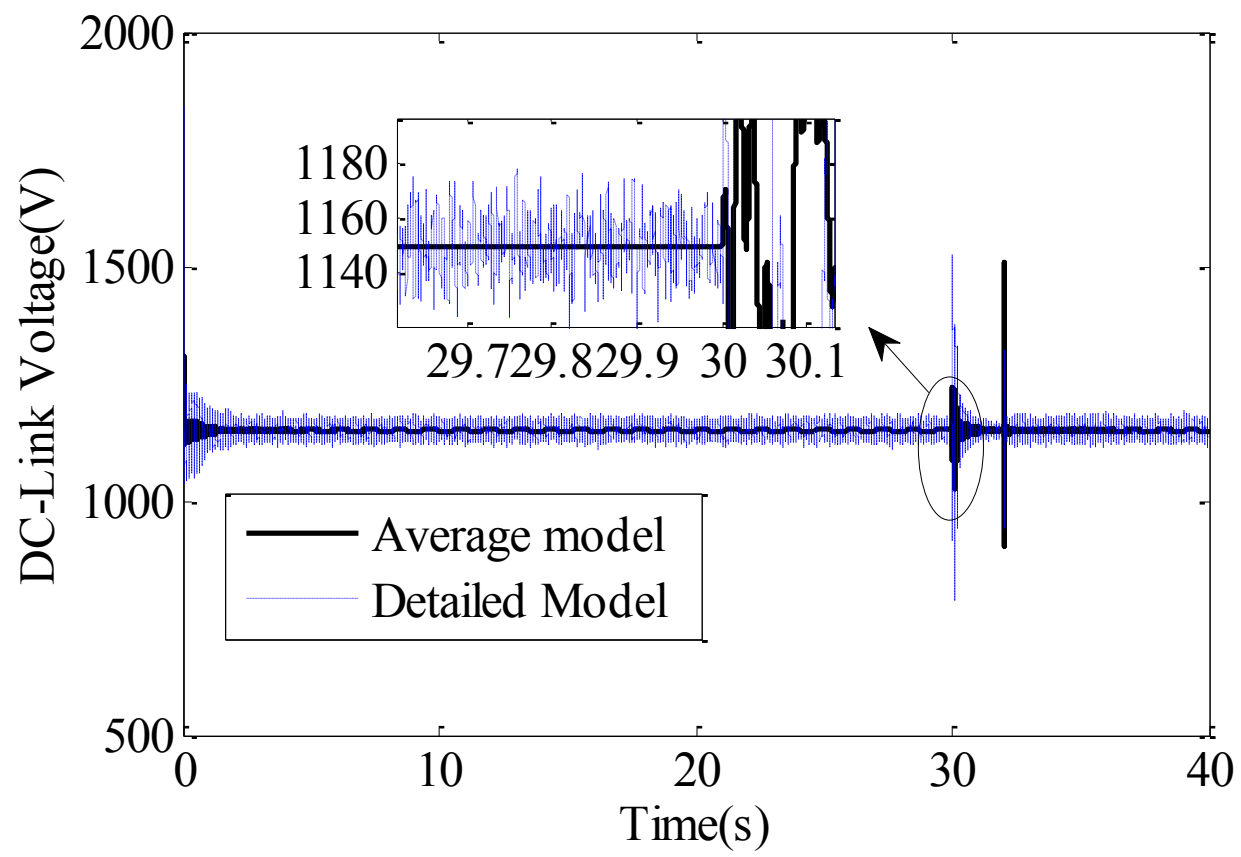

Figure 4.12: DC-Link voltage during three phase fault at the grid end 
From Figure 4.11 and 4.12 it can be observed that there is a dip in dc-link voltages during the grid fault and similarly there is a sudden change in the reactive power during the fault.

Finally generator speed is plotted during the fault condition in Figure 4.13.

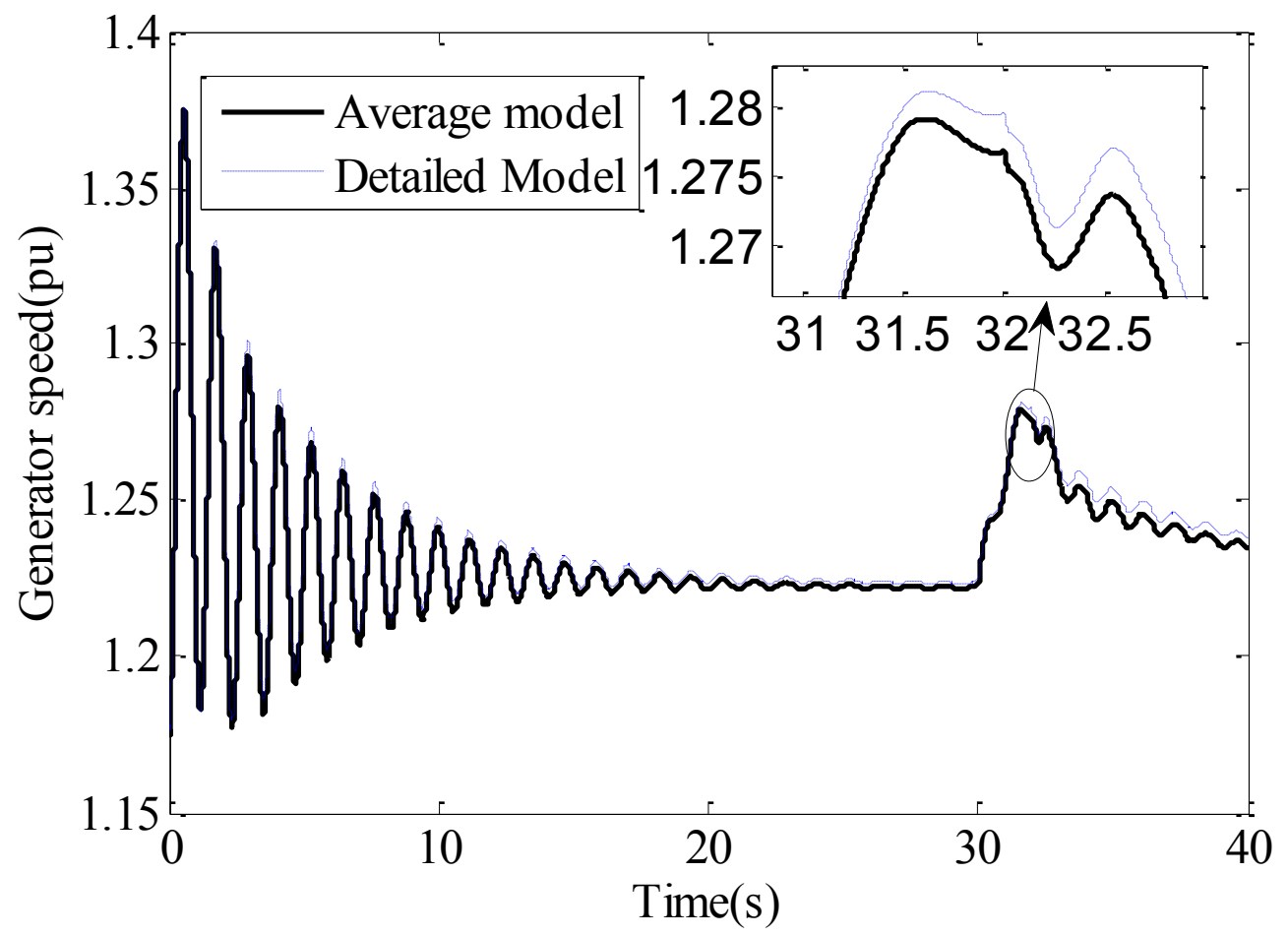

Figure 4.13: Generator speed during fault condition

During the fault condition a sudden change in generator speed can be observed in Figure 4.13. These plots prove that the developed system is responding to fault conditions.

Finally the developed model can be validated as the graphs for both the detailed and averaged model are similar and the averaged model is used for conduction small signal stability analysis which proved that the system is small signal stable.

\section{4 summary:}

In this chapter first a small signal model is developed for stability studies. In the second step simulations are performed for the large signal model which is derived in Chapter 2 and 3 . The developed model is validated by checking the simulations for the detailed and averaged model 
in the final step to check the system response to the fault, for this a three phase to ground fault is applied at the grid end. From small signal stability analysis the state variables which are most sensitive the stability are drawn. From the small signal model the observed states which are most effecting the system stability are $\left[\begin{array}{llllllll}\omega_{t} & x_{1} & i_{d r} & i_{q r} & i_{q s} & x_{7} & x_{2} & x_{4}\end{array}\right]$. To make the system more stable and to supply a stable power supply to the grid a power system stabiliser is introduced and its performance analysis is carried out in Chapter 5. For the observed states effecting the stability the PSS is designed and implemented at their corresponding points.

\subsection{Summary of equations from chapter 4:}

Aerodynamic small model:

$$
\begin{gathered}
2 H_{t} p\left(\Delta \omega_{t}\right)=-\left(\frac{-P_{t}}{\omega_{t 0}^{2}}-D_{t g}\right)\left(\Delta \omega_{t}\right)-K_{t g} \Delta\left(\theta_{t}-\theta_{r}\right)+D_{t g}\left(\Delta \omega_{r}\right) \\
p \Delta\left(\theta_{t}-\theta_{r}\right)=\left(\Delta \omega_{t}\right)-\left(\Delta \omega_{r}\right) \\
2 H_{g} p\left(\Delta \omega_{r}\right)=K_{t g} \Delta\left(\theta_{t}-\theta_{r}\right)+D_{t g}\left(\Delta \omega_{t}\right)-D_{t g}\left(\Delta \omega_{r}\right)-L_{m} i_{q s 0}\left(\Delta i_{d r}\right)-L_{m} i_{d r 0}\left(\Delta i_{q s}\right) \\
+L_{m} i_{d s 0}\left(\Delta i_{q r}\right)+L_{m} i_{q r 0}\left(\Delta i_{d s}\right)
\end{gathered}
$$

DFIG small signal model:

$$
\begin{gathered}
\quad \frac{L_{s}}{\omega_{b}} p\left(\Delta i_{d s}\right)+\frac{L_{m}}{\omega_{b}} p\left(\Delta i_{d r}\right)=\left(\Delta v_{d s}\right)+\frac{\omega_{e}}{\omega_{b}} L_{s}\left(\Delta i_{q s}\right)-R_{s}\left(\Delta i_{d s}\right)+\frac{\omega_{e}}{\omega_{b}} L_{m}\left(\Delta i_{q r}\right) \\
\quad \frac{L_{s}}{\omega_{b}} p\left(\Delta i_{q s}\right)+\frac{L_{m}}{\omega_{b}} p\left(\Delta i_{q r}\right)=\left(\Delta v_{q s}\right)-R_{s}\left(\Delta i_{q s}\right)-\frac{\omega_{e}}{\omega_{b}} L_{s}\left(\Delta i_{d s}\right)-\frac{\omega_{e}}{\omega_{b}} L_{m}\left(\Delta i_{d r}\right) \\
\frac{L_{r}}{\omega_{b}} p\left(\Delta i_{d r}\right)+\frac{L_{m}}{\omega_{b}} p\left(\Delta i_{d s}\right)=\left(\Delta v_{d r}\right)+b_{1}\left(\Delta i_{q s}\right)-a_{1}\left(\Delta \omega_{r}\right)+c_{1}\left(\Delta i_{q r}\right)-R_{r}\left(\Delta i_{d r}\right) \\
\text { Where } a_{1}=\left(\frac{L_{m}}{\omega_{b}} i_{q s 0}+\frac{L_{r}}{\omega_{b}} i_{q r 0}\right) ; b_{1}=\frac{L_{m}}{\omega_{b}}\left(\omega_{e}-\omega_{r 0}\right) c_{1}=\frac{L_{r}}{\omega_{b}}\left(\omega_{e}-\omega_{r 0}\right) \\
\frac{L_{r}}{\omega_{b}} p\left(\Delta i_{q r}\right)+\frac{L_{m}}{\omega_{b}} p\left(\Delta i_{q s}\right)=\left(\Delta v_{q r}\right)-b_{1}\left(\Delta i_{d s}\right)+a_{2}\left(\Delta \omega_{r}\right)-c_{1}\left(\Delta i_{d r}\right)-R_{r}\left(\Delta i_{d r}\right)
\end{gathered}
$$


Where $a_{2}=\left(\frac{L_{m}}{\omega_{b}} i_{d s 0}+\frac{L_{r}}{\omega_{b}} i_{d r 0}\right)$

DC-link small signal model:

$$
C p\left(\Delta v_{d c}\right)=a_{3}\left(\Delta v_{d c}\right)+\frac{1}{v_{d c 0}}\left(b_{2}-c_{2}\right)
$$

Where $a_{3}=\frac{1}{v_{d c 0}^{2}}\left(v_{d r 0} i_{d r 0}+v_{q r 0} i_{q r 0}-\left(v_{d g 0} i_{d g 0}+v_{q g 0} i_{q g 0}\right)\right)$;

$$
\begin{gathered}
b_{2}=\left(v_{d g 0}\left(\Delta i_{d g}\right)+i_{d g 0}\left(\Delta v_{d g}\right)+v_{q g 0}\left(\Delta i_{q g}\right)+i_{q g 0}\left(\Delta v_{q g}\right)\right) \\
c_{2}=\left(v_{d r 0}\left(\Delta i_{d r}\right)+i_{d r 0}\left(\Delta v_{d r}\right)+v_{q r 0}\left(\Delta i_{q r}\right)+i_{q r 0}\left(\Delta v_{q r}\right)\right)
\end{gathered}
$$

Controller model:

$$
\begin{gathered}
p \Delta \theta_{p l l}=\Delta \omega_{p l l} \\
p\left(\Delta \omega_{p l l}\right)-k_{p p l l} p\left(\Delta v_{q s}\right)=k_{\text {ipll }}\left(\Delta v_{q s}\right)
\end{gathered}
$$

Rotor side controller large signal model:

$$
\begin{gathered}
p x_{1}=P_{s r e f}-P_{s} \\
p x_{2}=K_{p 1}\left(P_{s r e f}-P_{s}\right)+K_{i 1} x_{1}-i_{d r} \\
v_{d r}=K_{p 2} K_{p 1}\left(P_{\text {sref }}-P_{s}\right)+K_{p 2} K_{i 1} x_{1}-K_{p 2} i_{d r}+K_{i 2} x_{2}-\left(\omega_{s}-\omega_{r}\right) L_{r} i_{q r} \\
-\left(\omega_{s}-\omega_{r}\right) L_{m} i_{q s} \\
p x_{3}=Q_{s r e f}-Q_{s} \\
p x_{4}=K_{p 3}\left(Q_{s r e f}-Q_{s}\right)+K_{i 3} x_{3}-i_{q r} \\
v_{q r}=K_{p 4} K_{p 3}\left(Q_{s r e f}-Q_{s}\right)+K_{p 4} K_{i 3} x_{3}-K_{p 4} i_{q r}+K_{i 4} x_{4}+\left(\omega_{s}-\omega_{r}\right) L_{r} i_{d r} \\
-\left(\omega_{s}-\omega_{r}\right) L_{m} i_{d s}
\end{gathered}
$$

Grid side controller large signal model:

$$
\begin{gathered}
p x_{5}=V_{d c}^{*}-V_{d c} \\
p x_{6}=K_{p 5}\left(V_{d c}^{*}-V_{d c}\right)+K_{i 5} x_{5}-i_{d g}
\end{gathered}
$$




$$
\begin{gathered}
v_{d g}=K_{p 6} K_{p 5}\left(V_{d c}^{*}-V_{d c}\right)+K_{p 6} K_{i 5} x_{5}-K_{p 6} i_{d g}+K_{i 6} x_{6}+L_{g}\left(\omega_{p l l}\right) i_{q g}+v_{d s} \\
p x_{7}=Q_{g}^{*}-Q_{g} \\
p x_{8}=K_{p 7}\left(Q_{g}^{*}-Q_{g}\right)+K_{i 7} x_{7}-i_{q g} \\
v_{q g}=K_{p 8} K_{p 7}\left(Q_{g}^{*}-Q_{g}\right)+K_{p 8} K_{i 7} x_{7}-K_{p 8} i_{q g}+K_{i 8} x_{8}-L_{g}\left(\omega_{p l l}\right) i_{d g}
\end{gathered}
$$

Linearized model of the rotor side controller:

$$
\begin{aligned}
& p\left(\Delta x_{1}\right)=-\left(v_{d s 0}\left(\Delta i_{d s}\right)+i_{d s 0}\left(\Delta v_{d s}\right)+v_{q s 0}\left(\Delta i_{q s}\right)+i_{q s 0}\left(\Delta v_{q s}\right)\right) \\
& p\left(\Delta x_{2}\right)=-K_{p 1}\left(v_{d s 0}\left(\Delta i_{d s}\right)+i_{d s 0}\left(\Delta v_{d s}\right)+v_{q s 0}\left(\Delta i_{q s}\right)+i_{q s 0}\left(\Delta v_{q s}\right)\right)+K_{i 1} \Delta x_{1} \\
&-\Delta i_{d r} \\
& \Delta v_{d r}=-K_{p 2} K_{p 1}\left(v_{d s 0}\left(\Delta i_{d s}\right)+i_{d s 0}\left(\Delta v_{d s}\right)+v_{q s 0}\left(\Delta i_{q s}\right)+i_{q s 0}\left(\Delta v_{q s}\right)\right) \\
&+\left(L_{m}\left(\omega_{r 0}-\omega_{s}\right)-K_{p 2} K_{p 1} v_{q s 0}\right)\left(\Delta i_{q s}\right)+K_{p 2} K_{i 1} \Delta x_{1}-K_{p 2} \Delta i_{d r}+K_{i 2} \Delta x_{2} \\
& \quad-\left(\omega_{r 0}-\omega_{s}\right) L_{r} \Delta i_{q r}+L_{r}\left(i_{q r o}+i_{q s o}\right) \Delta \omega_{r} \\
& p\left(\Delta x_{3}\right)=-v_{d s 0}\left(\Delta i_{q s}\right)-i_{q s 0}\left(\Delta v_{d s}\right)+v_{q s 0}\left(\Delta i_{d s}\right)+i_{d s 0}\left(\Delta v_{q s}\right) \\
& \Delta\left(\Delta x_{4}\right)=K_{p 3}\left(-v_{d s 0}\left(\Delta i_{q s}\right)-i_{q s 0}\left(\Delta v_{d s}\right)+v_{q s 0}\left(\Delta i_{d s}\right)+i_{d s 0}\left(\Delta v_{q s}\right)\right)+K_{i 1} \Delta x_{3} \\
&- \Delta i_{q r} \\
& \Delta v_{q r}=K_{p 4} K_{p 3}\left(-v_{d s 0}\left(\Delta i_{q s}\right)-i_{q s 0}\left(\Delta v_{d s}\right)+v_{q s 0}\left(\Delta i_{d s}\right)+i_{d s 0}\left(\Delta v_{q s}\right)\right) \\
&+\left(L_{m}\left(\omega_{s}-\omega_{r 0}\right)+K_{p 4} K_{p 3} v_{q s 0}\right)\left(\Delta i_{d s}\right)+K_{p 4} K_{i 3} \Delta x_{3}-K_{p 4} \Delta i_{q r}+K_{i 4} \Delta x_{4} \\
&+\left(\omega_{s}-\omega_{r 0}\right) L_{r} \Delta i_{d r}-L_{r}\left(i_{d r o}+i_{d s o}\right) \Delta \omega_{r}
\end{aligned}
$$

Linearized model of the grid side controller:

$$
\begin{gathered}
p \Delta x_{5}=-\Delta V_{d c} \\
p \Delta x_{6}=-K_{p 5}\left(\Delta V_{d c}\right)+K_{i 5} \Delta x_{5}-\Delta i_{d g}
\end{gathered}
$$




$$
\begin{gathered}
\Delta v_{d g}=-K_{p 6} K_{p 5}\left(\Delta V_{d c}\right)+K_{p 6} K_{i 5} \Delta x_{5}-K_{p 6} \Delta i_{d g}+K_{i 6} \Delta x_{6}+L_{g}\left(\omega_{p l l 0}\right) \Delta i_{q g}+\Delta v_{d s} \\
+L_{g} i_{q g 0}\left(\Delta \omega_{p l l}\right) \\
p \Delta x_{7}=-\Delta Q_{g} \\
p \Delta x_{8}=-K_{p 7}\left(\Delta Q_{g}\right)+K_{i 7} \Delta x_{7}-\Delta i_{q g} \\
\Delta v_{q g}=\left(-K_{p 8} K_{p 7} v_{d s 0}-K_{p 8}\right)\left(\Delta i_{q g}\right)-K_{p 8} K_{p 7} i_{q g 0}\left(\Delta v_{d s}\right)+\left(K_{p 8} K_{p 7} v_{q s 0}-L_{g} \omega_{p l l 0}\right)\left(\Delta i_{d g}\right) \\
+K_{p 8} K_{p 7} i_{d g 0}\left(\Delta v_{q s}\right)+K_{p 8} K_{i 7} \Delta x_{7}-K_{p 8} i_{q g}+K_{i 8} \Delta x_{8} \\
-L_{g} i_{d g 0}\left(\Delta \omega_{p l l}\right)
\end{gathered}
$$

Where $\Delta Q_{g}=v_{d s 0} \Delta i_{q g}+i_{q g 0} \Delta v_{d s}-\left(v_{q s 0} \Delta i_{d g}+i_{d g 0} \Delta v_{q s}\right)$

Linearized grid equations:

$$
\begin{aligned}
& L p \Delta\left(i_{d s}+i_{d g}\right) \\
& =\Delta v_{d s}-r \Delta i_{d s}-r \Delta i_{d g}+\omega_{0} L \Delta i_{q s}+\omega_{0} L \Delta i_{q g}+L\left(i_{q s 0}+i_{q g 0}\right)\left(\Delta \omega_{p l l}\right) \\
& -E \sin \delta_{0}\left(\Delta \delta_{p l l}\right) \\
& \operatorname{Lp} \Delta\left(i_{q s}+i_{q g}\right) \\
& =\Delta v_{q s}-r \Delta i_{q s}-r \Delta i_{q g}-\omega_{0} L \Delta i_{d s}-\omega_{0} L \Delta i_{d g}-L\left(i_{d s 0}+i_{d g 0}\right)\left(\Delta \omega_{p l l}\right) \\
& -E \cos \delta_{0}\left(\Delta \delta_{p l l}\right) \\
& L_{g} p\left(\Delta i_{d g}\right)=\left(\Delta v_{d g}\right)-R_{g}\left(\Delta i_{d g}\right)+\omega_{0} L_{g}\left(\Delta i_{q g}\right)+L_{g} i_{q g 0}\left(\Delta \omega_{p l l}\right)-\Delta v_{d s} \\
& L_{g} p\left(\Delta i_{q g}\right)=\left(\Delta v_{q g}\right)-R_{g}\left(\Delta i_{q g}\right)-\omega_{0} L_{g}\left(\Delta i_{d g}\right)-L_{g} i_{d g 0}\left(\Delta \omega_{p l l}\right)-\Delta v_{q s}
\end{aligned}
$$




\section{Chapter 5: Performance Analysis of a Grid connected DFIG based Wind}

\section{Generation System with a Power System Stabilizer}

\subsection{Introduction:}

This chapter discusses the power system stabilizer (PSS) designed in this thesis work. In Chapter 4 the system is shown without any damping controller or PSS, and it can be observed from the time domain simulations that the oscillations are greater and the settling time for the system to be completely stable is also more. The other observations made without PSS is during the small signal stability analysis, where the state variables affect the stability of the system. In this chapter a PSS is developed to damp the oscillations. A small signal analysis is also carried out for the developed PSS model.

Section 5.2 describes the power system stabilizer and the need to introduce the PSS in a wind energy conversion system. The design aspects of the newly developed PSS is discussed in Section 5.3. In Section 5.4 the small signal analysis is performed on the developed PSS by discussing the effects and the improvements it has brought to the model detailed in Chapter 4. Finally the time domain analysis is performed on the system with the PSS introduced and the results obtained are compared with those of the system without PSS developed in Chapter 4. It is observed that the developed system with a PSS is able to provide a more stable supply to the grid without damped oscillations and with a much less settling time.

\subsection{Power System Stabilizer (PSS):}

In reference to [82], power system stability can be defined as the property of the power system that enables it to remain in a state of operating equilibrium under normal operating conditions and to regain an acceptable state of equilibrium after being subjected to a disturbance. The main function of the PSS is to improve the system dynamic performance by providing a stable 
power which is done by damping the oscillation so the signals provided to the network. In conventional power plants, the power system stabilizer is used for controlling the excitation system and it is mainly employed for synchronous generators.

The input signal to the PSS that are usually employed in the conventional methods are the shaft speed, terminal frequency and power [82], simply it can be said that auxiliary stabilizing signals are used as input signals for a PSS.

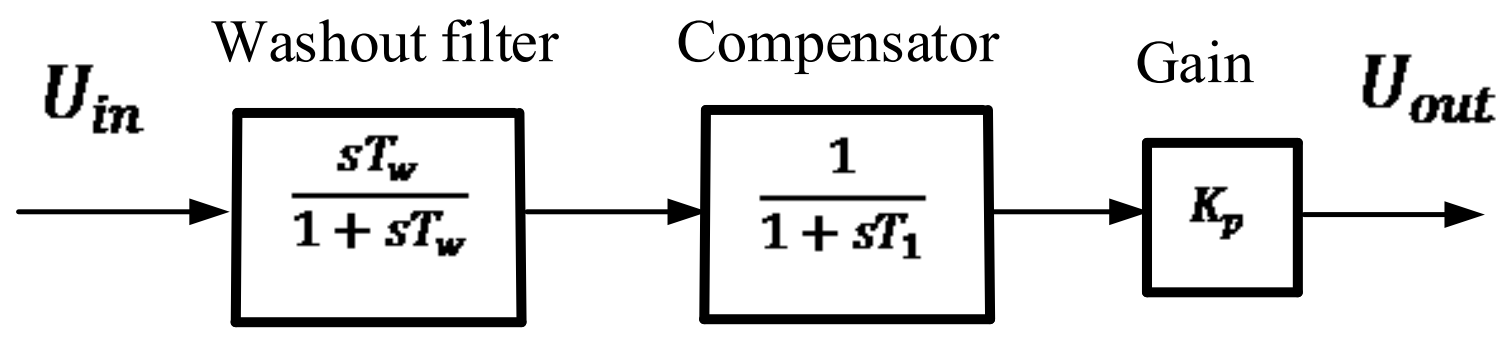

Figure 5.1: General structure of a power system stabilizer [82]

In general the structure of a power system stabilizer as shown in Figure 5.1 consists of a washout filter, a proportional gain or a PI controller and a compensator block. In conventional power plants where synchronous generators generate the power, in PSS design the compensator used is a phase compensator and it is mainly used to compensate for the phase lag between the input signal and the electrical torque and it is usually a first order block. Whereas the washout filter acts as a high pass filter and it is designed to respond only to the changes in the input signal provided to it, and this do not show much effect or changes in the final output signal like voltage signals. The range of the time constant in washout filter is given in the range of $1 s$ to $20 s$ [82], finally the gain determines the amount of damping introduced into PSS. As described in [82], the value of gain is taken as high as possible and it is observed that the damping increases with the increase in the gain and beyond certain maximum value the system damping started decreasing. The time constant and the gains chosen for the current 
research work are discussed and are given while presenting the design aspects of PSS in Section 5.3.

As discussed in Section 5.1 and 5.2, the PSS is mainly implemented in conventional power plants for synchronous generators, similar to this a PSS can be employed for a wind energy conversion system and there is a need for this, as a stable power with damped signals is required for a wind energy system to connect to the grid. Implementing PSS with wind energy conversion system is considered to be one of the new technologies in grid connected wind energy conversion system. It is mention in [41], that presence of PSS in DFIG system improves the damping of the oscillations in the network. So this thesis mainly focused on implementing new PSS for DFIG based wind energy conversion system and a complete analysis is carried out by showing the improvements achieved on employing PSS at required points in the system.

\subsection{Design and Small Signal Stability Analysis of a DFIG based Wind System with a Power System Stabilizer (PSS):}

In Chapter 4 the states effecting the stability are discussed, and in these states it is observed that the most common variables effecting the stability of the system are the stator voltages and the PLL frequency $\omega$. Even in time domain analysis it is observed that the oscillations are more due to these variables, so the PSS is employed at the points required, which are determined from small signal analysis. The newly designed PSS is able to damp the oscillations and even increase the stability of the system.

Two power system stabilizers are designed and implemented in this research work. As mentioned above the two input signals which are implemented for PSS are the stator voltage signal $v_{d q s}$ and the PLL frequency $\omega_{p l l}$. The two PSS's are implemented for the rotor control loops. The two PSS's are connected at the current reference signals of the rotor, i.e. at the input 
of the inner current control loops. The GEVS and GEFS which are introduced for a single DG system in [51] are taken as base for designing the two PSS's for current research work.

In this research work, both small signal analysis and the large signal time domain analysis is performed for the entire wind energy conversion system along with the PSS's. As mentioned in [82], proper care is taken to ensure that the overall system stability is enhanced, and not just the small signal stability analysis. So the system time domain analysis is carried out and is presented in Section 5.5.

\subsubsection{Design of voltage PSS:}

The overall control block diagram with a voltage stabilizer is given in Figure 5.2.

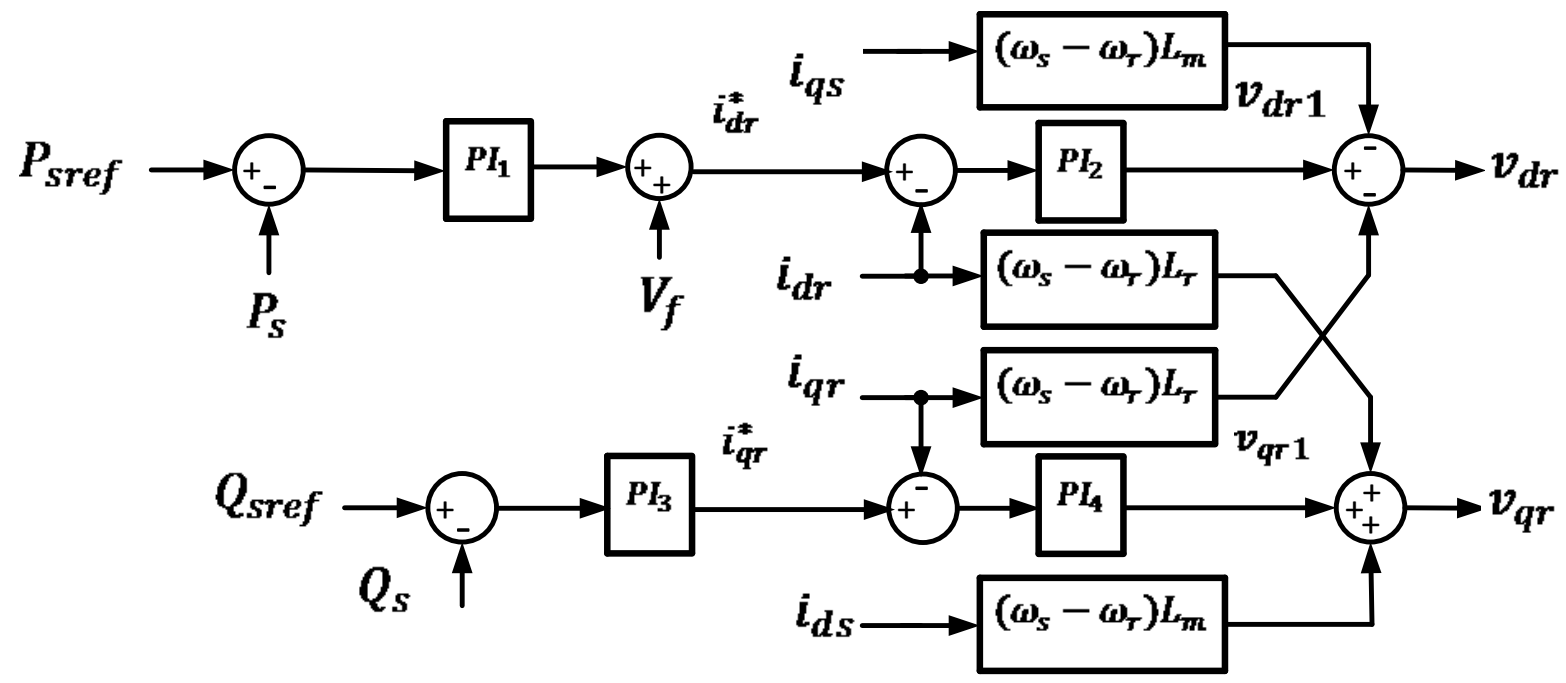

Figure 5.2: RSC with voltage PSS

In Figure 5.2 the additional signal to the rotor side controller used in Chapter 4 is the stabilized signal $V_{f}$. The voltage of the stator is fed to this signal, as the input signal and is passed through the washout filter and the compensator and finally the output signal is obtained from the proportional gain controller which acts as the damping factor. The block diagram for the stabilizer is shown in Figure 5.3. The final output signal $V_{f}$ is fed as the input signal to the inner current controller at the $i_{d r e f}$ point along with the controlled signal of the power. The 
reason for employing PSS at this point is explained clearly by observing the small signal stability analysis discussed below.

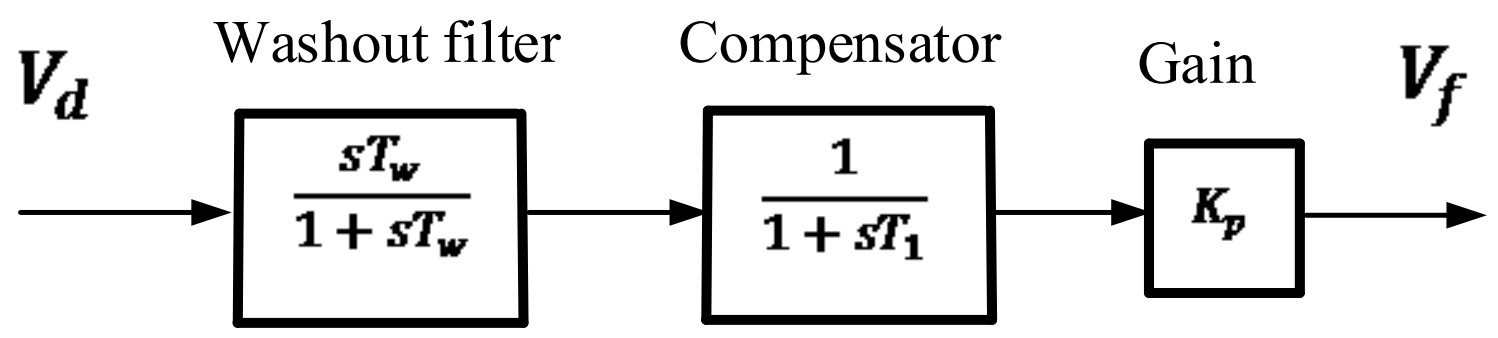

Figure 5.3: Diagram block for voltage PSS.

\subsubsection{Modeling of voltage stabilizer:}

From the Figure 5.3, the voltage stabilizer can be modeled and the mathematical model can be derived as:

$$
V_{f}=V_{d}\left(\frac{s T_{w}}{1+s T_{w}}\right)\left(\frac{1}{1+s T_{1}}\right)\left(K_{p}\right)
$$

In 5.1, $T_{w}$ is the time constant of the washout filter and $T_{1}$ is the time constant of the compensator; $K_{p}$ is the proportional gain and $V_{f}$ is the output signal of PSS which is added to the inner current control loop of the rotor side converter and finally $V_{d}$ is the stator voltage and it is modeled as given in 5.2.

$$
V_{d}=\sqrt{v_{d s}^{2}+v_{q s}^{2}}
$$

The block diagram given in Figure 5.3 and the mathematical models 5.1 and 5.2 are used for large signal time domain analysis. For developing the small signal model the same procedure is followed as mentioned in Chapter 4 of Section 4.2. All the linearized equation used for developing the small signal model and for analysis are taken from Chapter 4 for all the modules except for the rotor side current controller. In RSC the current $d$-reference frame is fed with the new voltage stabilizer signal which is given in Figure 5.3 


\subsubsection{Small signal stability analysis with voltage PSS:}

The small signal analysis is carried out by adding this block and the linearized equations for

5.1 can be derived and it is expressed as given below:

$$
\left(\Delta V_{f}\right)+\left(T_{w}+T_{1}\right) p\left(\Delta V_{f}\right)=T_{w} K_{p} p\left(\Delta V_{d}\right)
$$

The eigenvalues of $(E, A)$ obtained with the developed PSS are shown in Table 5.1.

Table 5.1: Eigenvalues of $(E, A)$ of grid connected DFIG ystem with voltage stabilizer

\begin{tabular}{|c|c|c|c|c|c|}
\hline & $\lambda=\sigma \pm j \omega$ & $\sigma$ & $\omega$ & $\xi$ & $f_{\text {osc }}$ \\
\hline$\lambda_{1}, \lambda_{2}$ & $-738.50 \pm 890.7 i$ & -738.50 & 890.7 & 0.63 & 141.7 \\
\hline$\lambda_{3}$ & -403.03 & -403.03 & 0 & 1 & 0 \\
\hline$\lambda_{4}, \lambda_{5}$ & $-4.6 \pm 376.47 i$ & -4.6 & 376.47 & 0.012 & 59.9 \\
\hline$\lambda_{6}, \lambda_{7}$ & $-69.85 \pm 364.88 i$ & -69.85 & 364.88 & 0.18 & 58.07 \\
\hline$\lambda_{8}, \lambda_{9}$ & $-57.67 \pm 30.03 i$ & -57.67 & 30.03 & 0.88 & 4.7 \\
\hline$\lambda_{10}$ & -40.67 & -40.67 & 0 & 1 & 0 \\
\hline$\lambda_{11}$ & -28.09 & -28.09 & 0 & 1 & 0 \\
\hline$\lambda_{12}, \lambda_{13}$ & $-9.45 \pm 8.39 i$ & -9.45 & 8.39 & 0.74 & 1.33 \\
\hline$\lambda_{14}$ & -0.07 & -0.07 & 0 & 1 & 0 \\
\hline$\lambda_{15}, \lambda_{16}$ & $-0.51 \pm 0.7 i$ & -0.51 & 0.7 & 0.5812 & 0.1114 \\
\hline$\lambda_{17}, \lambda_{18}$ & $-6.09 \pm 0.16 i$ & -6.09 & 0.16 & 0.9 & 0.025 \\
\hline$\lambda_{19}$ & -0.9304 & -0.9304 & 0 & 1 & 0 \\
\hline$\lambda_{20}$ & -0.93012 & -0.93012 & 0 & 1 & 0 \\
\hline$\lambda_{21}$ & -11.14 & -11.14 & 0 & 1 & 0 \\
\hline
\end{tabular}


From Table 5.1 the small signal stability can be analyzed. Figure 5.4, shows the Eigen plot for the model, in this figure plot (b) represents the zoomed portion of the eigenvalues of $(E, A)$.

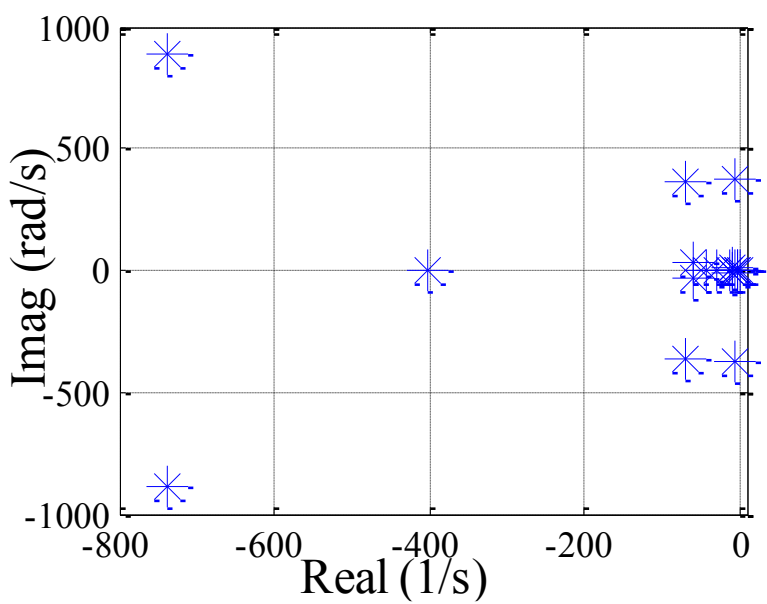

$a$

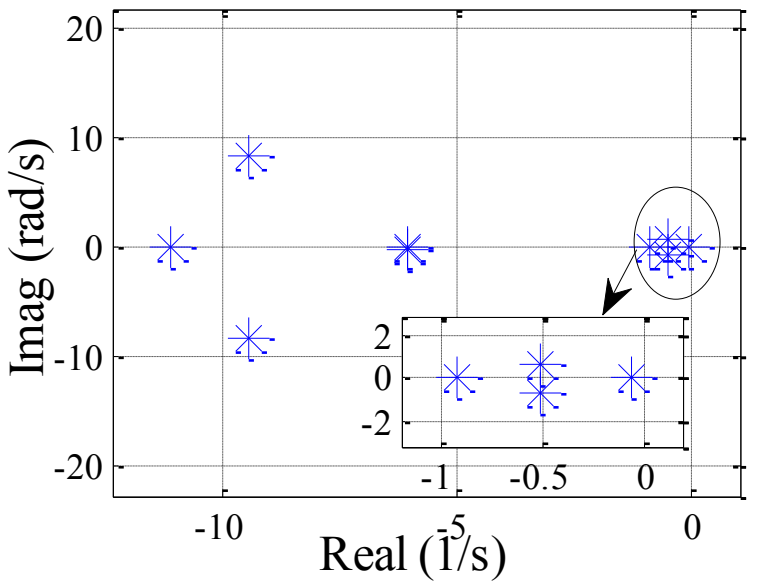

$b$

Figure 5.4: Eigen plot with voltage stabilizer

By adding a voltage stabilizer the eigenvalues of $(E, A)$ that are obtained can be observed in Table 5.1 and the table with corresponding state variables is given in Table 5.2. All of the state variables described in equation (4.47) of Chapter 4 are same even in this model except the new state variable added i.e. the output signal of the voltage stabilizer $V_{f}$. As discussed this new stabilizer signal is added at the inner current controller loop of PSS along with the power signal. From Table 5.2, it can be observed that the real parts of some of the eigenvalues of $(E, A)$ have decreased towards negative part, which shows that the system has become more stable. This can be better understood by comparing the state variable Table 4.5 in chapter 4 with the Table 5.2 in this chapter. The light color indicates the variables that have increased with negative real parts and the thick color indicates the newly added variable of the stabilizer i.e. $V_{f}$. On adding the voltage stabilizer the state variables which are effected and which attained more stability are $i_{d r}, i_{q r}, i_{q s}, x_{7}, v_{d s}$ and $\theta_{p l l}$. These are the main state variables that 
need to be improved and the developed stabilizer is successful in achieving more stable system with desired outputs. The large signal model and effect of the voltage stabilizer is discussed Section 5.4.

Table 5.2: State variables corresponding to eigenvalues of $(E, A)$

\begin{tabular}{|c|c|c|}
\hline & $\lambda=\sigma \pm j \omega$ & State Variables $\Delta X$ \\
\hline$\lambda_{1}, \lambda_{2}$ & $-738.50 \pm 890.7 i$ & $i_{d g}$ and $i_{q g}$ \\
\hline$\lambda_{3}$ & -403.03 & $i_{d s}$ \\
\hline$\lambda_{4}, \lambda_{5}$ & $-4.6 \pm 376.47 i$ & $i_{d r}$ and $i_{q r}$ \\
\hline$\lambda_{6}, \lambda_{7}$ & $-69.85 \pm 364.88 i$ & $i_{q s}$ and $x_{7}$ \\
\hline$\lambda_{8}, \lambda_{9}$ & $-57.67 \pm 30.03 i$ & $V_{d c}$ and $x_{5}$ \\
\hline$\lambda_{10}$ & -40.67 & $x_{3}$ \\
\hline$\lambda_{11}$ & -28.09 & $x_{1}$ \\
\hline$\lambda_{12}, \lambda_{13}$ & $-9.45 \pm 8.39 i$ & $v_{q s}$ and $\theta_{p l l}$ \\
\hline$\lambda_{14}$ & -0.07 & $\omega_{t}$ \\
\hline$\lambda_{15}, \lambda_{16}$ & $-0.51 \pm 0.7 i$ & $\omega_{r}$ and $\theta_{t w}$ \\
\hline$\lambda_{17}, \lambda_{18}$ & $-6.09 \pm 0.16 i$ & $x_{6}$ and $x_{8}$ \\
\hline$\lambda_{19}$ & -0.9304 & $x_{4}$ \\
\hline$\lambda_{20}$ & -0.93012 & $x_{2}$ \\
\hline$\lambda_{21}$ & -11.14 & $V_{f}$ \\
\hline
\end{tabular}

The time constants and the proportional gain is determined from small signal analysis. It is observed that $T_{w}$ and $T_{1}$ has same value which is equal to 1 , beyond this value the system becomes unstable, therefore the maximum time constant for the washout filter for the PSS with 
voltage as input is taken, similarly the proportional gain value is observed for different values and the system finally attained stability with $K_{p}=5$.

From the small signal mode discussed above and the large signal of the system with voltage PSS, which will be described in Section 5.4 it is observed that some of the desired output is not achieved at the maximum extent and with this PSS there is control over only some parts of the system. From Figure 5.2, it is clear that the voltage stabilizer is fed only to the $d$-axis reference frame of the RSC, so due to this, there is no proper control on the $q$-axis reference frame. To solve this issue a new stabilizer is developed with transformation technique to attain a full control and stable system and it is discussed in Section 5.3.4.

\subsubsection{Modeling of PSS with transformation technique:}

As discussed above the transformation technique is implemented on the inner current controller of RSC on both $d$ and $q$ axis control loops. The block diagram with transformation technique is given in Figure 5.5:

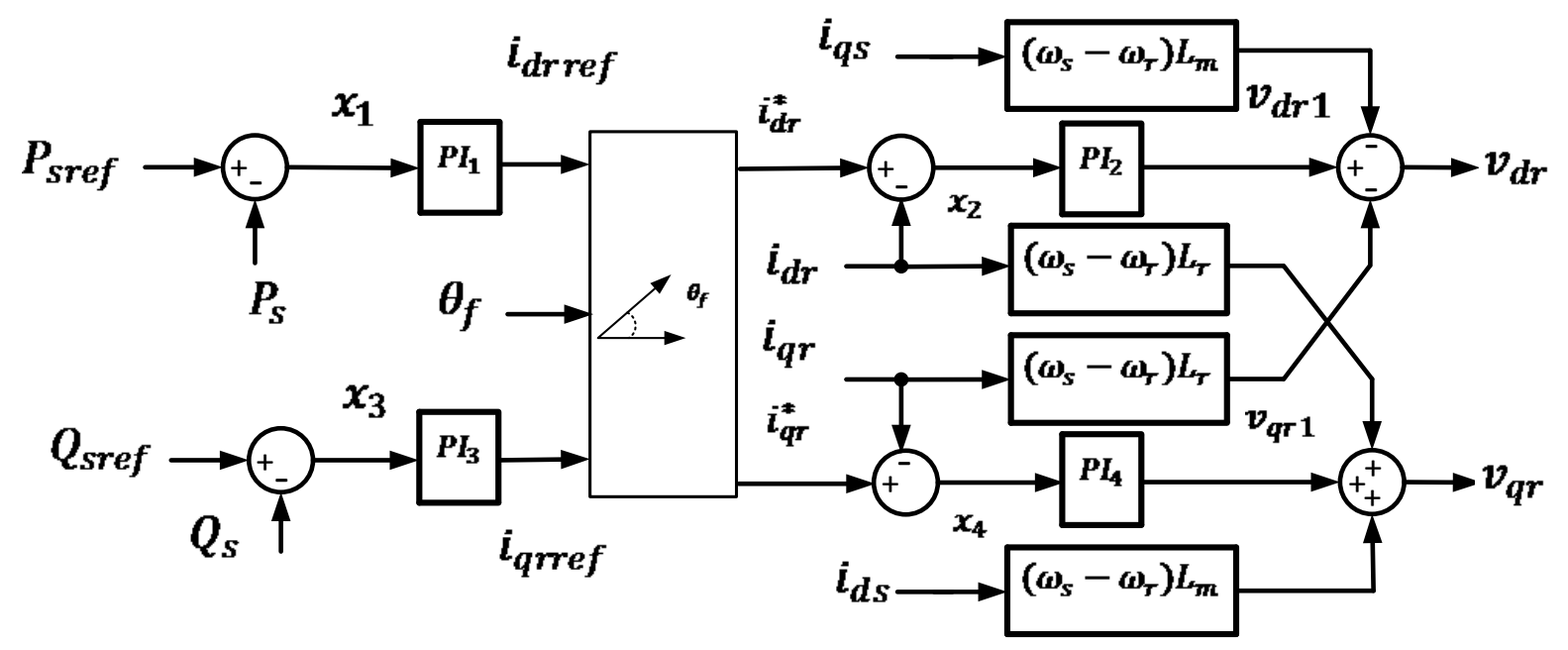

Figure 5.5: RSC with transformation PSS 
It can be observed form the Figure 5.5, the transformation technique is implemented for both $d$ and $q$ axis. The signal $\theta_{f}$ is obtained from PLL frequency which is represented as $\omega_{p l l}$. The block diagram for generating $\theta_{f}$ is given in Figure 5.6.

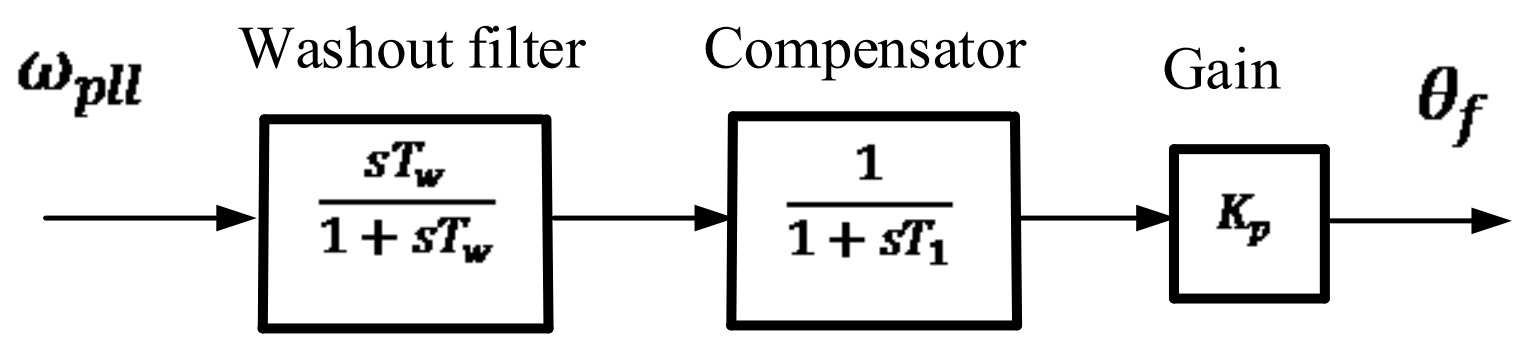

Figure 5.6: Block diagram for PSS with transformation technique.

Mathematical modeling for signal $\theta_{f}$ can be given as:

$$
\theta_{f}=\left(K_{t} \omega_{p l l}\right)\left(\frac{s T_{w}}{1+s T_{w}}\right)\left(\frac{1}{1+s T_{1}}\right)
$$

The transformation is defined as:

$$
\begin{aligned}
& i_{d r}^{*}=\cos \left(\theta_{f}\right) i_{d r r e f}-\sin \left(\theta_{f}\right) i_{\text {qrref }} \\
& i_{q r}^{*}=\sin \left(\theta_{f}\right) i_{d r r e f}+\cos \left(\theta_{f}\right) i_{q r r e f}
\end{aligned}
$$

All the constant values are determined from small signal analysis. Once the transformation angle signal $\theta_{f}$ is obtained it is fed to the reference current blocks, since the reference currents $i_{d r}^{*}$ and $i_{q r}^{*}$ are transformed to currents $i_{d r r e f}$ and $i_{q r r e f}$ through the transformation block as discussed in [51]. As mentioned, the transformation technique discussed in [51] is implemented for a DG system but, in this thesis it is implemented for a complete grid connected DFIG based wind generating system and all the analysis with DFIG based wind system is discussed in this thesis work. It can be seen from Figure 5.5, that the new reference signals to the current controller loops are transformed and they are $i_{d r}^{*}$ and $i_{q r}^{*}$. The other signals in (5.5) and (5.6) are the $i_{d r r e f}$ and $i_{\text {qrref }}$ these signals can be obtained from the power 
control block shown in Figure 5.5. This contribute to the large signal model. The small signal model is developed by linearizing the above equations (5.4) - (5.6). The small signal model is developed by linearizing the above equations and by following the same procedure discussed in Chapter 4.

\subsubsection{Small signal model with PSS transformation technique:}

On linearizing (5.4) - (5.6) the linearized model obtained is given as:

$$
\begin{gathered}
\Delta \theta_{f}+T_{w} p\left(\Delta \theta_{f}\right)+T_{1} p\left(\Delta \theta_{f}\right)=K_{t} T_{w} p\left(\Delta \omega_{p l l}\right) \\
\Delta i_{d r}^{*}=\cos \theta_{f 0} \Delta i_{d r r e f}-\sin \theta_{f 0} i_{q r r e f}-i_{q 0} \Delta \theta_{f} \\
\Delta i_{q r}^{*}=\sin \theta_{f 0} \Delta i_{d r r e f}+\cos \theta_{f 0} i_{q r r e f}+i_{d 0} \Delta \theta_{f}
\end{gathered}
$$

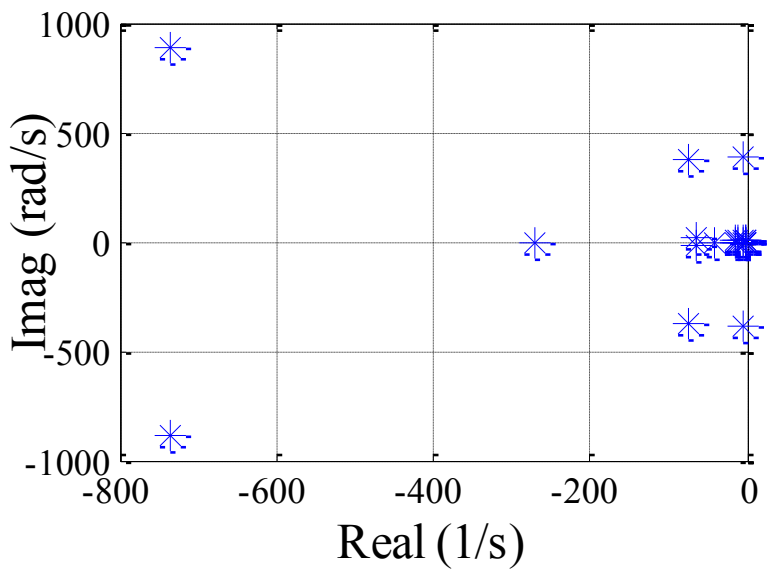

$a$

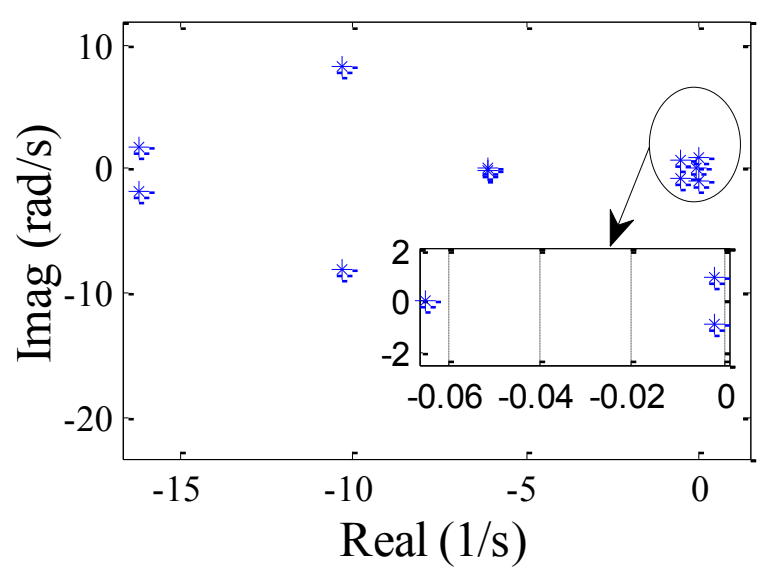

$b$

Figure 5.7: Eigen plot with PSS

In Figure 5.7, plot (a) represents the eigenvalues of $(E, A)$ obtained for the model with transformation technique power system stabilizer, whereas plot (b) represents the zoomed plot for the Eigen values shown in plot (a). Here in this case the input given to the power system stabilizer is the frequency $\omega_{\text {pll }}$. A clear description about the plot and the Eigen values obtained is discussed below. 
The plot obtained after performing small signal analysis is given in Figure 5.7. From this figure it can be observed that there is a shift in the Eigen plot. The shift is away from the imaginary axis and even the real negative parts of the system also increased. This indicates that the system has reached a higher level of stability. The main difference between the system with voltage power system stabilizer and the system with the transformation technique power system stabilizer is that there is full control over the stability of the system with transformation PSS which is fed at both axis. This statement can be supported by observing the Table 5.3 and Table 5.1 which describes the eigenvalues of $(E, A)$.

Table 5.3: Eigenvalues of $(E, A)$ of the grid connected DFIG ystem with transformation PSS

\begin{tabular}{|c|c|c|c|c|c|}
\hline & $\lambda=\sigma \pm j \omega$ & $\sigma$ & $\omega$ & $\xi$ & $f_{\text {osc }}$ \\
\hline$\lambda_{1}, \lambda_{2}$ & $-735.81 \pm 897.7 i$ & -735.81 & 897.7 & 0.63 & 142.1 \\
\hline$\lambda_{3}$ & -269.8 & -269.8 & 0 & 1 & 0 \\
\hline$\lambda_{4}, \lambda_{5}$ & $-5.02 \pm 389.67 i$ & -5.02 & 389.67 & 0.0129 & 62.07 \\
\hline$\lambda_{6}, \lambda_{7}$ & $-73.54 \pm 376.36 i$ & -73.54 & 376 & 0.19 & 59.9 \\
\hline$\lambda_{8}, \lambda_{9}$ & $-64 \pm 37.08 i$ & -64.19 & 37.08 & 0.86 & 5.9 \\
\hline$\lambda_{10}$ & -40.67 & -40.67 & 0 & 1 & 0 \\
\hline$\lambda_{12}, \lambda_{13}$ & $-10.34 \pm 8.47 i$ & -10.34 & 8.47 & 0.77 & 1.35 \\
\hline$\lambda_{14}$ & -0.07 & -0.07 & 0 & 1 & 0 \\
\hline$\lambda_{15}, \lambda_{16}$ & $-0.51 \pm 0.7 i$ & -0.51 & 0.7 & 0.5889 & 0.1114 \\
\hline$\lambda_{17}, \lambda_{18}$ & $-6.09 \pm 0.16 i$ & -6.09 & 0.16 & 0.9 & 0.026 \\
\hline$\lambda_{19}, \lambda_{20}$ & $-0.0019 \pm 0.93 i$ & -0.0019 & 0.93 & 0.002 & 0.15 \\
\hline$\lambda_{11}, \lambda_{21}$ & $-16.16 \pm 1.79 i$ & -16.16 & 1.79 & 0.99 & 0.28 \\
\hline
\end{tabular}


Table 5.4, describes the corresponding state variables for the value obtained in Table 5.3.

Table 5.4: State variables corresponding to eigenvalues of $(E, A)$

\begin{tabular}{|c|c|c|}
\hline & $\lambda=\sigma \pm j \omega$ & State Variables $\Delta X$ \\
\hline$\lambda_{1}, \lambda_{2}$ & $-735.81 \pm 897.7 i$ & $i_{d g}$ and $i_{q g}$ \\
\hline$\lambda_{3}$ & -269.8 & $i_{d s}$ \\
\hline$\lambda_{4}, \lambda_{5}$ & $-5.02 \pm 389.67 i$ & $i_{d r}$ and $i_{q r}$ \\
\hline$\lambda_{6}, \lambda_{7}$ & $-73.54 \pm 376.36 i$ & $i_{q s}$ and $x_{7}$ \\
\hline$\lambda_{8}, \lambda_{9}$ & $-64 \pm 37.08 i$ & $V_{d c}$ and $x_{5}$ \\
\hline$\lambda_{10}$ & -40.67 & $x_{3}$ \\
\hline$\lambda_{12}, \lambda_{13}$ & $-10.34 \pm 8.47 i$ & $v_{q s}$ and $\theta_{p l l}$ \\
\hline$\lambda_{14}$ & -0.07 & $\omega_{t}$ \\
\hline$\lambda_{15}, \lambda_{16}$ & $-0.51 \pm 0.7 i$ & $\omega_{r}$ and $\theta_{t w}$ \\
\hline$\lambda_{17}, \lambda_{18}$ & $-6.09 \pm 0.16 i$ & $x_{6}$ and $x_{8}$ \\
\hline$\lambda_{19}, \lambda_{20}$ & $-0.0019 \pm 0.93 i$ & $x_{2}, x_{4}$ \\
\hline$\lambda_{11}, \lambda_{21}$ & $-16.16 \pm 1.79 i$ & $x_{1}$ and $\theta_{f}$ \\
\hline
\end{tabular}

The stability attained when implementing the system with the transformed PSS can be better understood by comparing the Table 5.4 with Table 4.5 described in Chapter 4 . The first observation is that there is a greater shift of the eigenvalues of $(E, A)$ away from the imaginary axis and the next observation is that on implementing the system with PSS, some of the negative real numbers are replaced by the complex numbers by attaining more stability. The light coloured eigenvalues of $(E, A)$ and the corresponding state variables are shown in Table 5.4, and these light colored indicate the state variables which attained more stability. The dark 
coloured state variable is the newly added signal input from the PSS. As the signal is added at the $x_{1}$ and $x_{4}$ states there is change in the state variables related to these states, along with the change in the states which are interrelated to these states. Finally the system obtained is more stable when compared to the previous two models. The time constants for the washout filter and compensator are determined from the small signal model by checking the stability of the system. The parameters are given in appendix B. The state variables which are need to be improved in stability have attained the desired stability by performing this PSS. The large signal model and the comparison with the model without PSS is discussed in Section 5.4.

\subsection{Large Signal Analysis with PSS:}

In this section large signals analysis is performed for both the PSS's developed Section 5.3. The obtained outputs with the PSS are compared without PSS obtained in Chapter 4.

\section{Results for voltage power system stabilizer:}
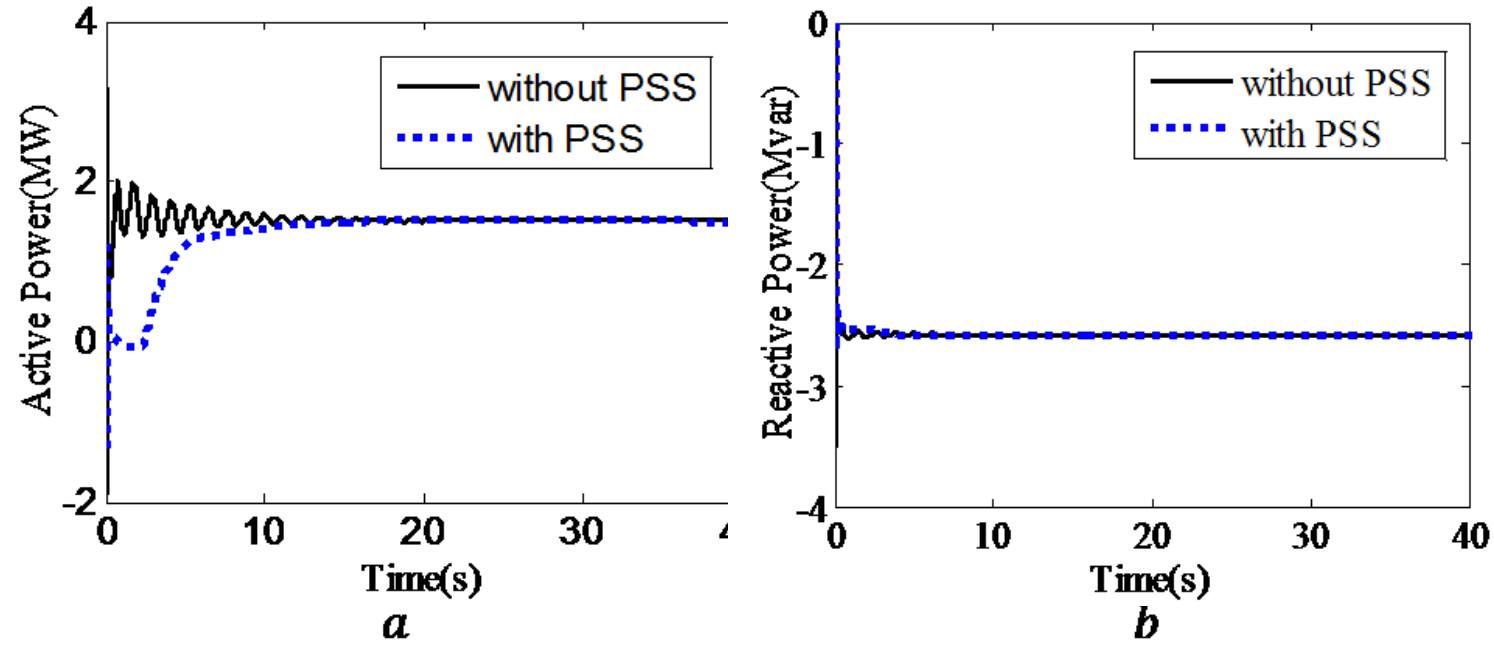

Figure 5.8: (a).Active power and (b).reactive power with and without PSS

Figure 5.8 represents the plot obtained by employing voltage PSS discussed in Section 5.3.2 to the wind energy system. Figure5.8 (a), represents active power and 5.8(b) represents plot 
obtained for reactive power. The think coloured waveform indicates the waveform obtained without PSS and the dotted line plot indicate the plot obtained for voltage PSS. From this figure it can be noticed that the damping of the active power are increased and a stable power is supplied to the grid. Similarly the waveforms for DC-Link voltage and generator speed are given in Figure 5.9.
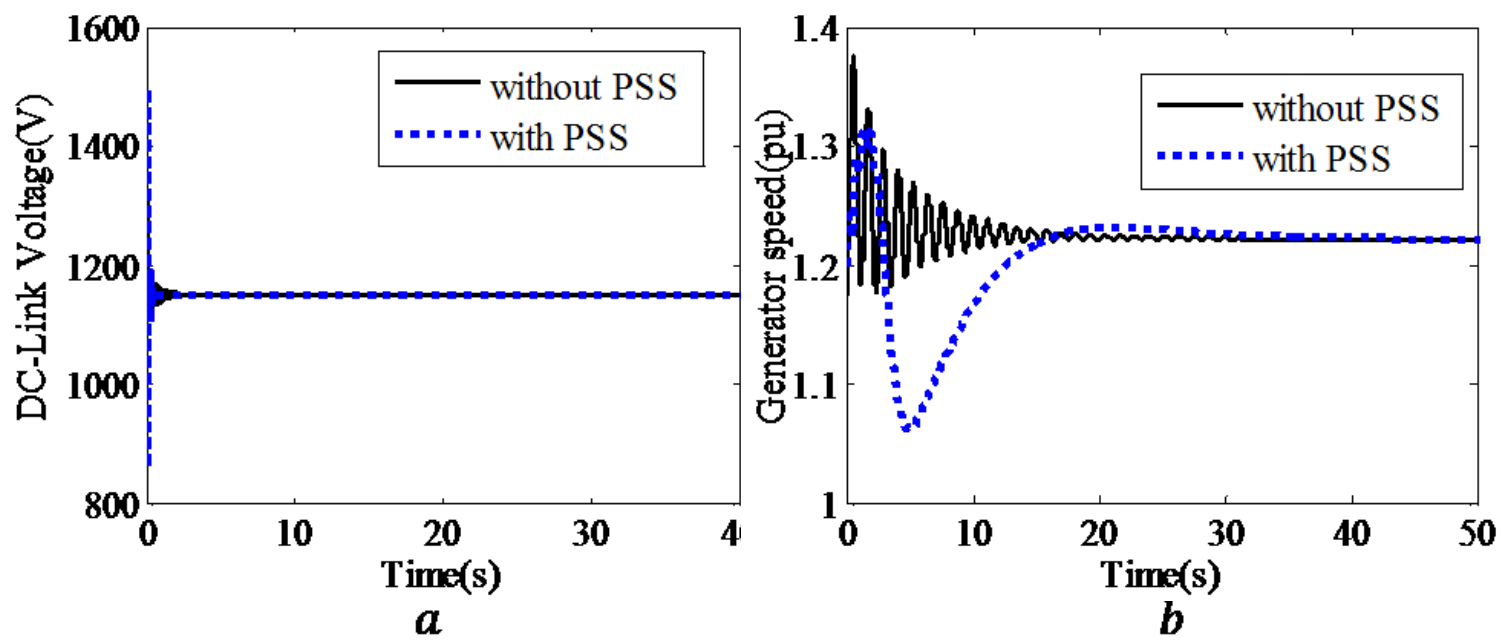

Figure 5.9: (a). DC-link voltage and (b). Generator speed

Figure 5.9 (a) shows the plot for the DC-link voltage and 5.9 (b) shows the plot for the generator speed. The increase in damping resulting from the use of the voltage power system stabilizer can be observed from the plot. Therefore, it can be concluded that when the designed voltage power system stabilizer is employed, the system achieves the desired outputs and is even small signal stable. From Figure 5.9(b) which represents the generator speed it can be observed that there are some deviations in the generator speed with voltage PSS. From time period $\tau=20 \mathrm{~s}$ to $\tau=40 \mathrm{~s}$, there is some deviation and the system is not able to give the complete desired output with voltage PSS, to overcome this problem and to achieve a complete stable system a new PSS i.e. the power system stabilizer with transformation technique is designed and is implemented for the system. 


\section{Results for power system stabilizer with transformation technique:}

As disused above and in Section 5.3.3, the system with voltage power system stabilizer is having some drawbacks and these can be eliminated by using the transformation technique to the system and this is observed from the small signal model and the large signal model which is discussed in this section.
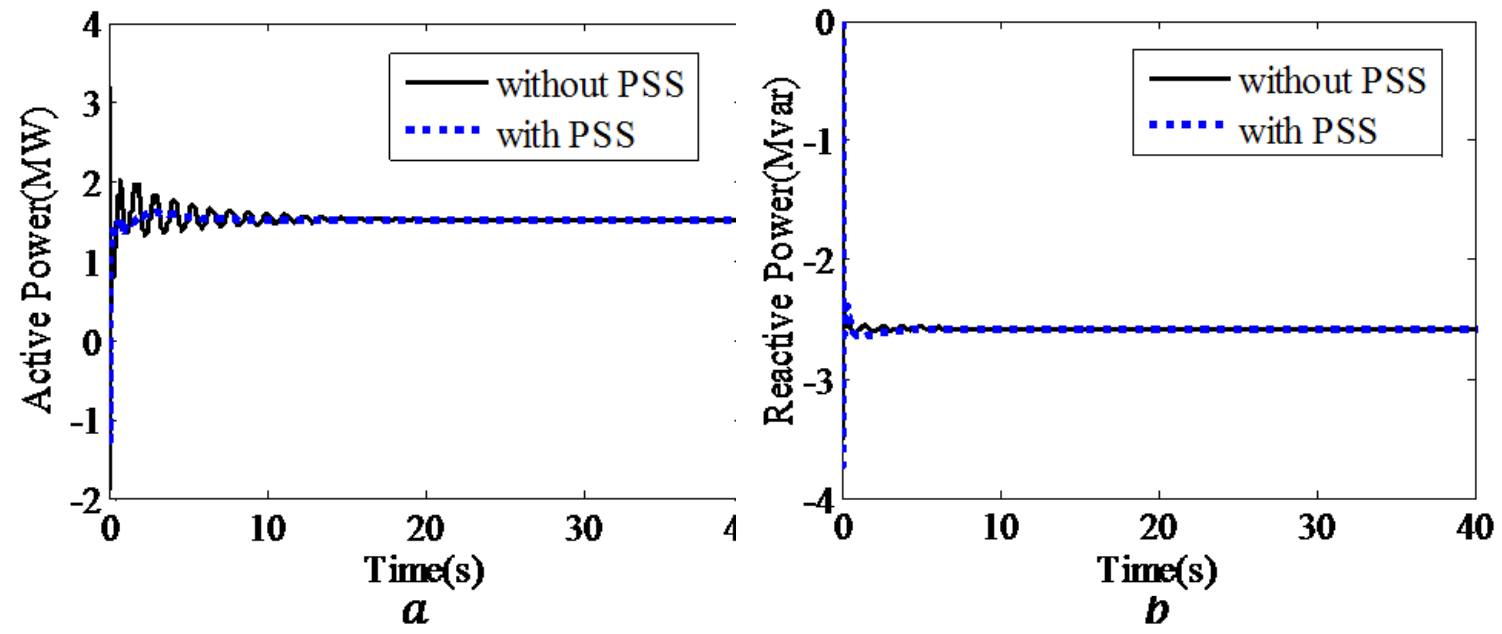

Figure 5.10: (a).Active power and (b).reactive power with and without PSS
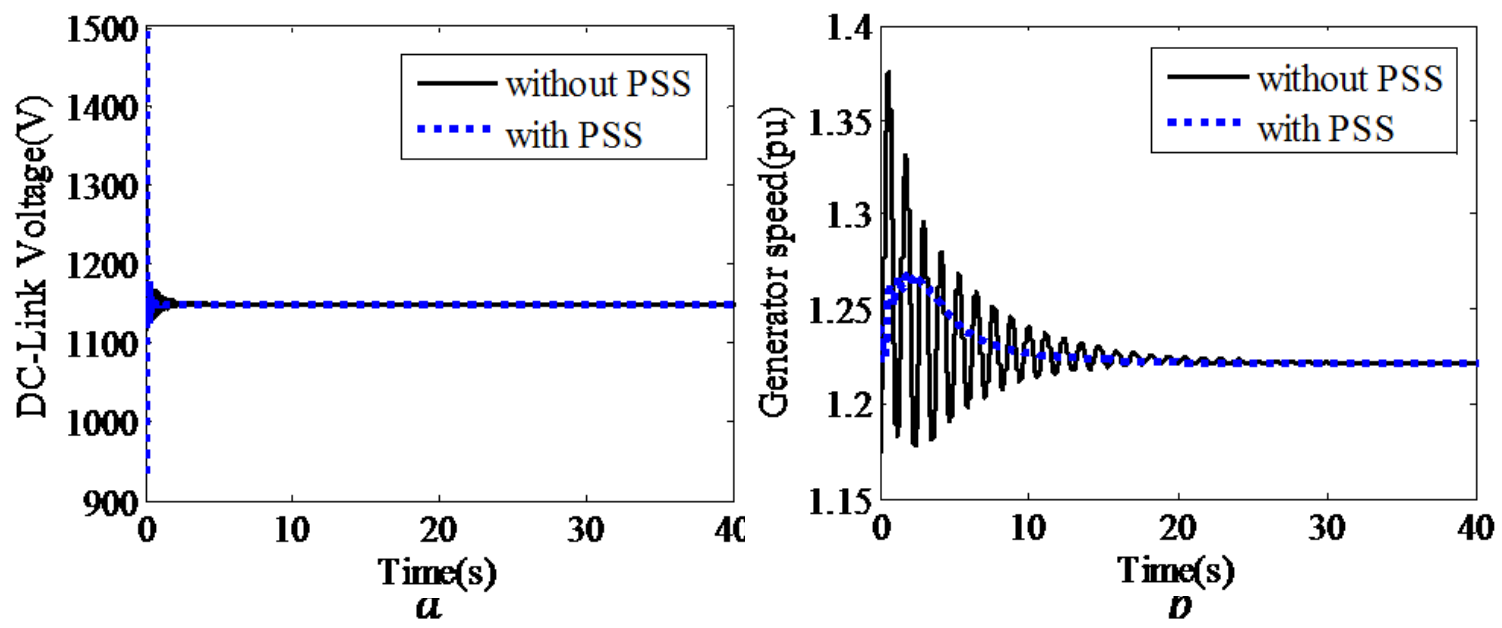

Figure 5.11: (a). DC-link voltage and (b). Generator speed with PSS 
In Figure 5.10, (a) represents the plot for active power and figure (b) represents the plot for reactive power with PSS and without PSS. The plot with PSS is for transformation PSS. Figure 5.11 (a) indicates the plot for DC-link voltage and 5.10 (b) represents the plot for generator speed. From the Figure 5.10, it can be observe that the active power plot is increased in its damping. Similarly from Figure 5.11(b), the plot for generator speed, it can be observed that there is an increase in damping the oscillations. When compared with the voltage power system stabilizer the PSS with transformation technique achieved good damping and a stable power is given to the grid both in the small signal model and the large signal model.

Fault analysis with voltage PSS:
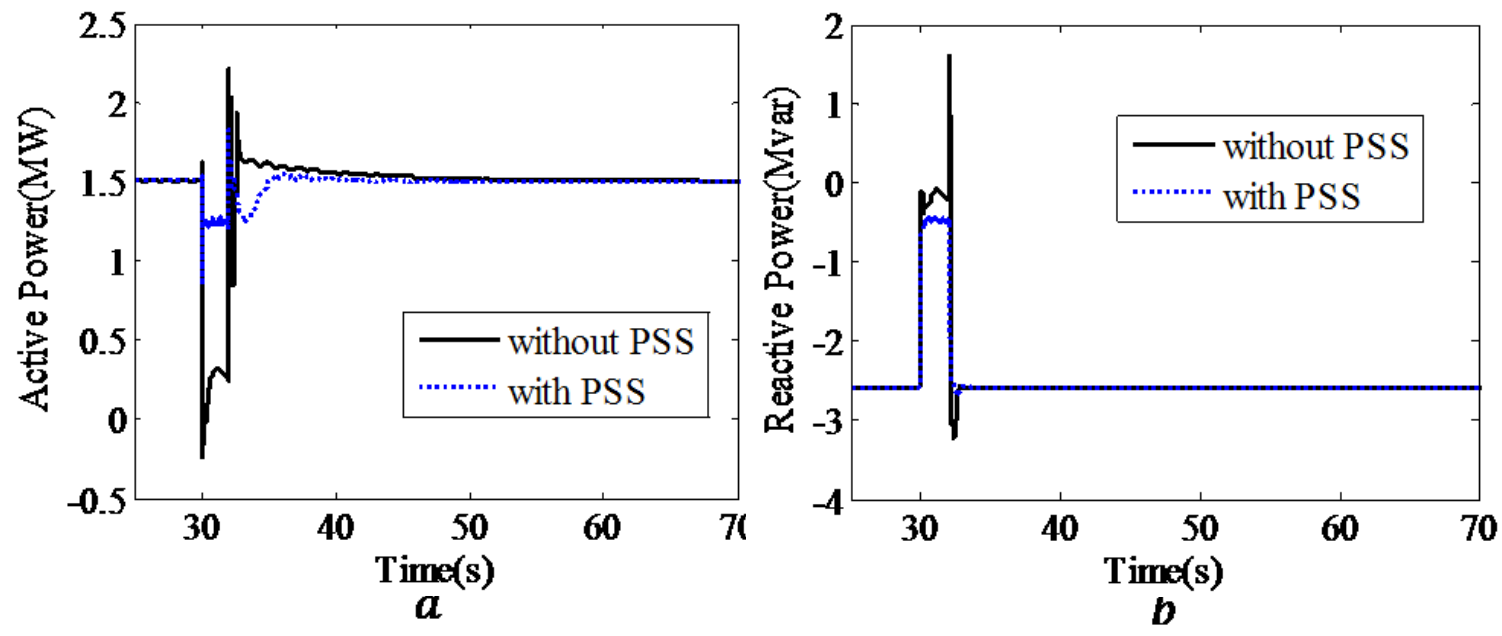

Figure 5.12: (a).Active power and (b).reactive power with and without PSS with fault For fault analysis a three phase fault is applied to the system for a time period of $\tau=2 s$ between times $\tau=30$ s to $\tau=32 s$. The results obtained for active power and reactive power are shown on Figure 5.12 (a) and (b) respectively. From the above plot it can be observed that the PSS with voltage as input is able to damp the oscillations during the fault and the settling time after fault is also very less. 
The plot for DC-link voltage and generator speed during the fault is shown in Figure 5.13.
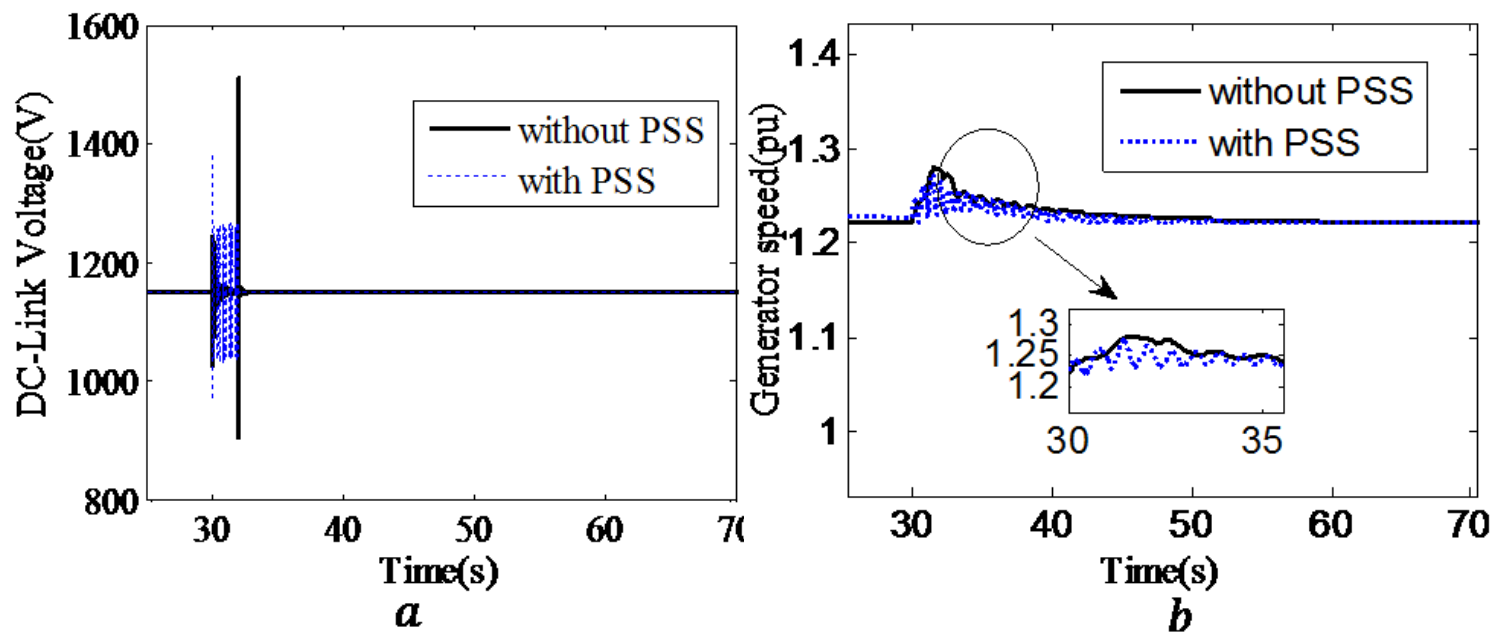

Figure 5.13: (a). DC-link voltage and (b). Generator speed with PSS during fault condition The effect of voltage PSS on DC- link voltage and generator speed during the fault condition can be observed from Figure 5.13. The oscillations of the generator speed during the fault condition are damped and the settling time is also less.

Fault analysis with transformation PSS:
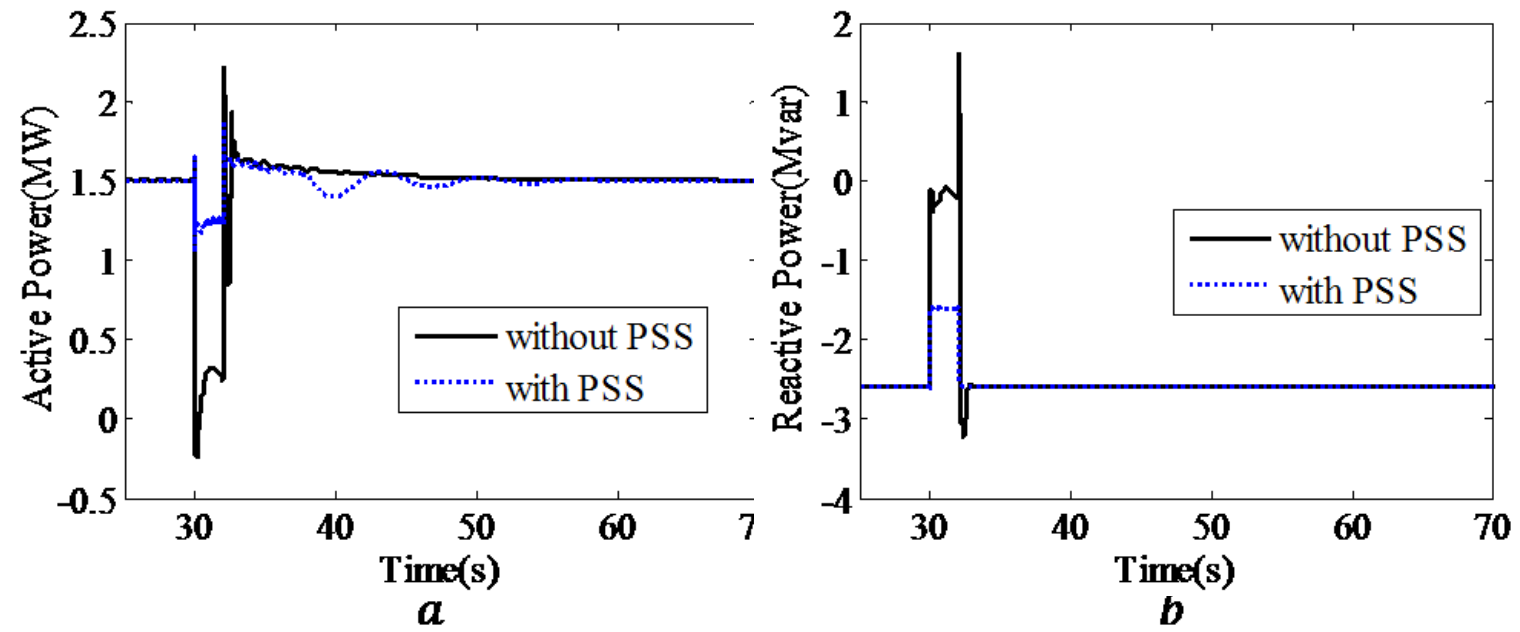

Figure 5.14: (a).Active power and (b).reactive power with and without PSS during fault condition 
The Figure 5.14, represents the plot for active and reactive power for the PSS with transformation technique. The comparison is made with and without PSS. The plot for DClink voltage and generator speed are given in Figure 5.15 (a) and (b) respectively.
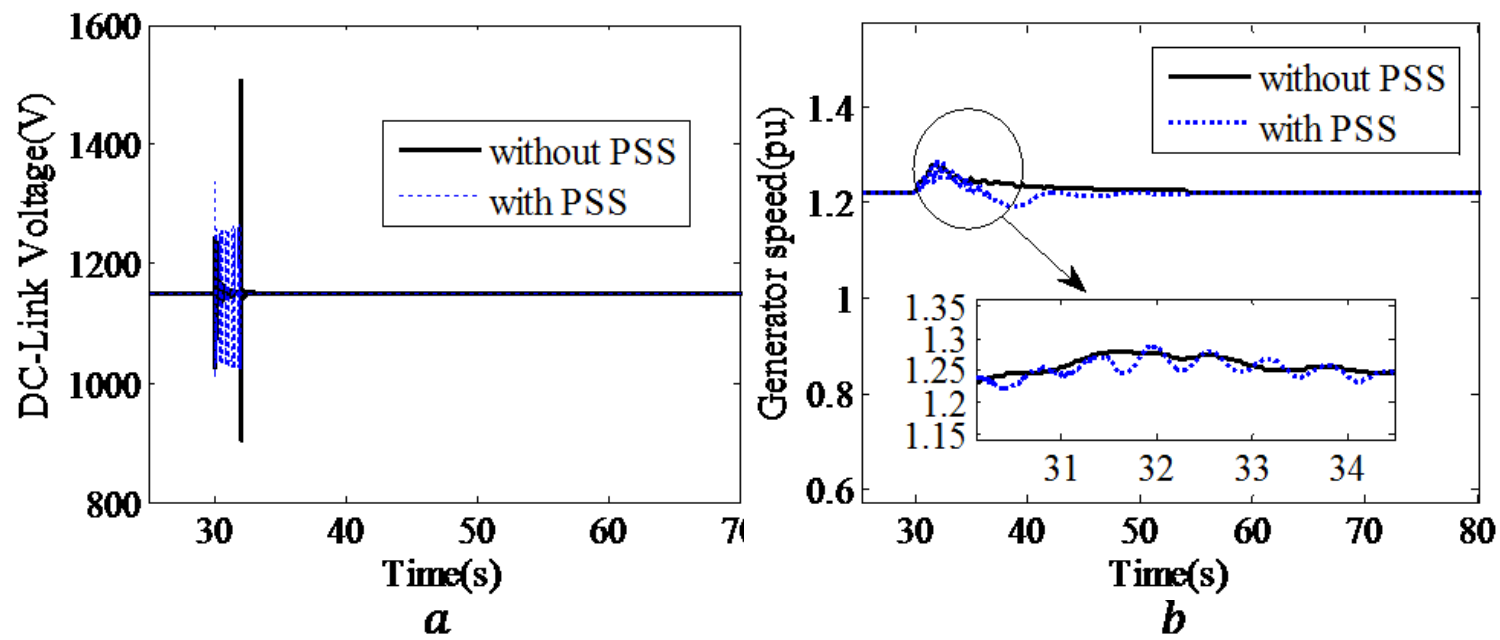

Figure 5.15: (a). DC-link voltage and (b). Generator speed with PSS during fault condition

Figures 5.14 and 5.15 shows the plot with transformation PSS during fault condition. It can be observed that the oscillations are damped even during the fault condition. This can be more clearly observed from the power plot and the speed plot in which the oscillations are damped the settling time is also very less. From this discussion it can be concluded that both the PSS's are working effectively during the fault condition.

\subsection{Summary:}

In this chapter the two power system stabilizers are designed and they are discussed, the two power system stabilizers are the voltage power system stabilizer and the power system stabilizer with transformation technique. The complete analysis i.e. the small signal and the large signal analysis as discussed in Chapter 4 is carried out for the entire system by employing both the PSS's. The PSS's with the voltage and transformation techniques are able to provide a stable power to the grid and they are successful in improving the damping of the oscillations. 
A fault analysis is also carried out during the large signal analysis and the performance of the PSS's during the fault condition are discussed. The improvements in the system's performance when employing PSS to the grid integrated DFIG wind generation system are discussed. 


\section{Chapter 6: Conclusions and Future Work}

\subsection{Conclusions:}

In the field of renewable energy, wind energy is one of the fastest growing and considered as the promising technology for electric power generation. From the literature review in Chapter 1, it is noticed that the contribution of wind farms to electric power grid continues to increase, majorly the DFIG based wind farms. For this reason the current research work mainly focused on DFIG based wind generation system. When integrating a wind farm into the grid one of the major issues that needs to be considered is the power system stability. By considering power system stability as the major challenge, this thesis developed a set of power system stabilizers and investigated the impact of the designed PSS's on the grid integrated DFIG based wind generation system. From the investigated results it is observed that the designed power system stabilizers provide a better performance to supply a stable power to the grid.

The main contributions and conclusions of this research work are summarized as follows:

- A thorough literature review is conducted on the different available power system stabilizers and the damping controllers that are implemented to DFIG based wind generation system. From the literature survey it is noticed that very few power systems stabilizers and damping controllers are implemented to DFIG based wind generation systems, since employing PSS's for a wind generation system is a very new technology. So the main focus of this current research work is done on investigating the performance analysis of the designed two power system stabilizers. The results obtained are compared to those of wind generation system without power system stabilizer.

- In the first step the complete mathematical modeling of the DFIG based wind generation system is carried out for all the modules of the system. Then the modeling of all the 
controllers is presented. The two main electrical controllers are the RSC and GSC. Bothe the controllers are developed based on voltage oriented vector control strategy. Implementing voltage control oriented control strategy for both the RSC and GSC is new and this included in this research work, along with a complete analysis.

- In the second step, the analyses of the developed system are carried out without any PSS. The two analyses that are performed are the small signal stability analysis and the time domain analysis. The small signal stability analysis is carried out for the averaged wind model. A thorough investigation of the results obtained for the small signal model is conducted, and the state variables that are most sensitive are noticed. The large signal analysis is carried put for the averaged model and the switching (detailed) model without PSS. From the analysis it is observed that the results obtained from time domain analysis for the averaged model and the switching model are similar. So from this observation the developed model is verified and validated. Furthermore, time domain analysis is carried out for the averaged and switching model by introducing a fault for a time period $\tau=2 \mathrm{~s}$ between $\tau=30 s$ to $\tau=32 s$. The waveforms for both the models are the same.

- The observation made from the time domain analysis is that the oscillations are very high during the initial conditions and during the fault analysis it is observed that the oscillations are very high during the fault and that the post-fault recovery time is high.

- To overcome the challenges observed during the small signal stability analysis and the time domain analysis, two PSS' are added for the developed system. The two power system stabilizers vary based on their input from each other. For one of the PSS stator voltage is taken as the input and for the other PSS grid frequency is taken as input and both of them are implemented at the input of the inner current control loop of RSC. The PSS with voltage 
as input is fed only to the $d$ - axis frame whereas the PSS with frequency as input is fed to both the axis $(d q)$. Thee PSS with stator voltage as input is able to improve the system stability to some extent in both the small signal and time domain analysis. On the other hand, the PSS with frequency as input is able to deliver desired results in both small signal and time domain analysis.

- During fault studies the PSS with frequency as input i.e. with transformation technique is able to improve the damping of the oscillations and it is observed that the post-fault recovery time is much lower. The PSS with stator voltage as input is also able to give a good performance but not as high as that of the PSS with frequency as an input.

Finally it can be concluded that the system performance is increased by employing any of the two PSS's. However the PSS with frequency as an input with transformation technique yields better results and better performance.

\subsection{Suggestions for Future Work:}

Based on the results of the present thesis work, the following points are identified as potential for future work:

- For further analysis the present research work can be extended by employing some protection techniques, for example crowbar protection.

- New control techniques can be derived to improve the performance of the DFIG system with unsymmetrical faults.

- In this research work the controllers used are the conventional PI controllers. The artificial intelligence (AI) techniques can be employed to design the controllers.

- For further complete real time analysis, the developed system can be validated through lab validation or with field validation techniques. 
- The developed system is using a single wind generation system, it can be developed to a number of wind farms and an aggregated wind farm can be developed and further more analysis can be carried out with the aggregated model.

- An additional analysis can also be carried out for a hybrid renewable power plant, i.e. integrating both PV and the developed wind system with PSS. 


\section{Appendix A: DFIG based wind generation system with parallel RLC load}

In this section the DFIG based wind system discussed in Chapter 2 is extended with parallel $R L C$ load. The system with parallel $R L C$ load can be observed form Figure A.1

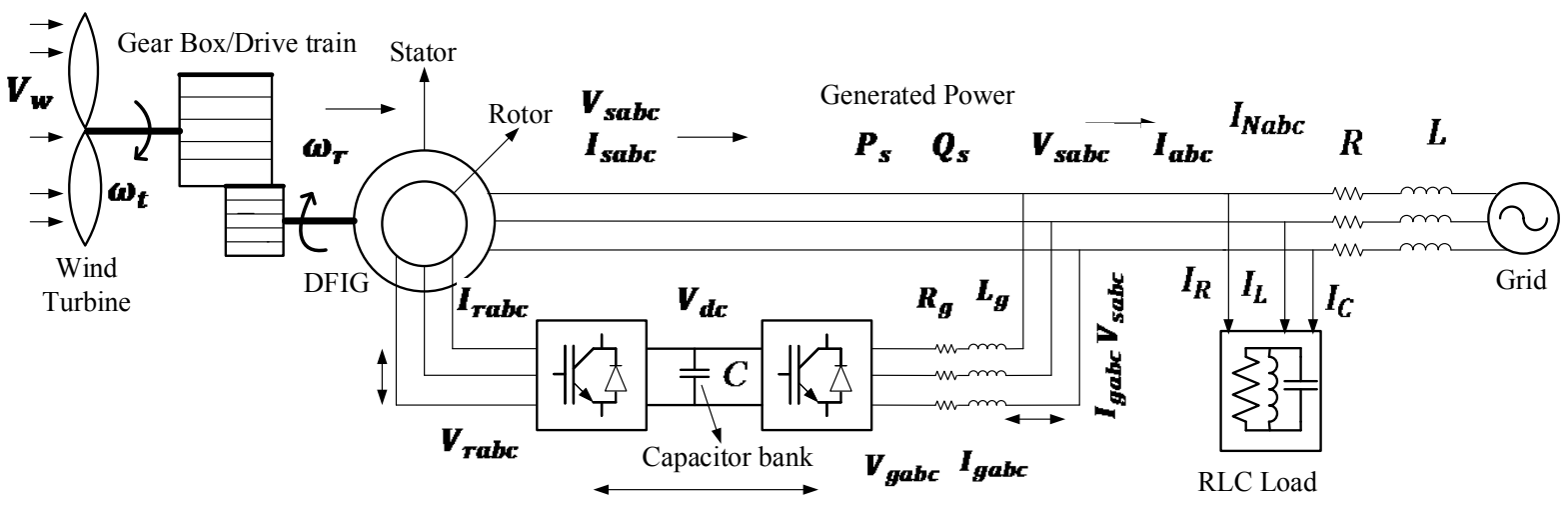

Figure A.1: System with parallel RLC load.

From the above figure the system can be modeled as follows:

$$
\begin{gathered}
I_{a b c}=I_{s a b c}+I_{g a b c} \\
I_{N a b c}=I_{a b c}-\left(I_{R a b c}+I_{L a b c}+I_{C a b c}\right) \\
v_{s a b c}=R I_{N a b c}+L p I_{N a b c}+e_{a b c} \\
I_{R a b c}=\frac{V_{s a b c}}{R_{l}} \\
v_{s a b c}=L_{l} p I_{L a b c} \\
I_{c a b c}=C_{l} p v_{s a b c} \\
V_{g a b c}=R_{g} I_{g a b c}+L_{g} \frac{d}{d t} I_{g a b c}+v_{s a b c}
\end{gathered}
$$

Model for parallel $R L C$ load in $d q$ reference frame:

$$
\begin{gathered}
v_{s d}=R I_{N d}+L p I_{N d}-\omega_{p l l} L I_{N q}+e_{d} \\
v_{s q}=R I_{N q}+L p I_{N q}+\omega_{p l l} L I_{N d}+e_{q} \\
I_{d q}=I_{s d q}+I_{d q g}
\end{gathered}
$$




$$
\begin{gathered}
i_{d q}=I_{R d q}+I_{L d q}+I_{C d q}+I_{N d q} \\
I_{R d q}=\frac{V_{s d q}}{R_{l}} \\
v_{s d}=L_{l} p I_{L d}-\omega_{p l l} L_{l} I_{L q} \\
v_{s Q}=L_{l} p I_{L q}+\omega_{p l l} L_{l} I_{L d} \\
I_{C d}=C_{l} p v_{s d}-\omega_{p l l} C_{l} v_{s q} \\
I_{C q}=C_{l} p v_{s q}+\omega_{p l l} C_{l} v_{s d} \\
V_{d g}=R_{g} I_{d g}+L_{g} p I_{d g}-\omega_{p l l} L_{g} I_{q g}+v_{d s} \\
V_{q g}=R_{g} I_{q g}+L_{g} p I_{q g}+\omega_{p l l} L_{g} I_{d g}+v_{q s}
\end{gathered}
$$

The parameters for this model is given in Appendix B. 


\section{Appendix B: Parameters for the system:}

To represent any electrical system, the common practice is to represent the parameters in per unit values. Representing the system in per unit values makes the complex system simpler for computations and for understanding. The main task to represent the system in per unit values is to define the base quantities. In a wind energy generation system, the convenient way of choosing base values is by taking the generator rated power, the nominal voltage, and the generator synchronous speed as the chosen base values.

\section{Base quantities:}

$S_{\text {base }}=1.5 ;(M V A)-$ Generator rated power

$V_{\text {base }}=120 \sqrt{2} ;(V)-$ Stator rated voltage

$\omega_{\text {base }}=2 p i 60 ;(\mathrm{rad} / \mathrm{s})-$ Electrical base speed

$I_{\text {base }}=\left(\frac{2}{3}\right)\left(\frac{S_{\text {base }}}{V_{\text {base }}}\right) ;(A)$-Base current

$Z_{\text {base }}=\left(\frac{V_{\text {base }}}{I_{\text {base }}}\right) ;(\Omega)-$ Base impedance

$L_{\text {base }}=\left(\frac{Z_{\text {base }}}{\omega_{\text {base }}}\right) ;(H)$-Base inductance

$\Psi_{\text {base }}=L_{\text {base }} I_{\text {base }} ;(w b-$ turns $)-$ Base flux

$P_{\text {base }}, Q_{\text {base }}=S_{\text {base }} ;(W),(V A r)-$ Base active and reactive power

\section{DFIG Parameters:}

$\omega_{s}=1[p u]-$ Synchronous speed

$r_{s}=0.00706[\mathrm{pu}]-$ Stator resistance

$r_{r}=0.005[p u]-$ Rotor resistance

$L_{l s}=0.171[p u]$ - Stator inductance

$L_{l r}=0.156[p u]-$ Rotor inductance 
$L_{m}=2.9[p u]-$ Mutual inductance

$H_{g}=0.9 s$ - Generator inertia constant

$p=3$ - No of pairs of poles

Drive train data:

$H_{t}=4.32 \mathrm{~s}-$ Wind turbine inertia constant

$K_{t g}=1.11-$ Shaft spring constant

$D_{t g}=1.5-$ Shaft mutual damping

$V_{w}=15[\mathrm{~m} / \mathrm{s}]$ - rated wind speed

$R=33.3$ Blade length

$\rho=1.225\left[\mathrm{~kg} / \mathrm{m}^{3}\right]-$ Air density

$\omega_{\text {trated }}=\frac{V_{\text {wrated }} \lambda_{\text {optimal }}}{R}[\mathrm{rad} / \mathrm{s}]$ Turbine rated speed

$\lambda_{\text {optimal }}=9.94$ Tip speed ratio

$C_{p \max }=0.5-$ Maximum value of $C_{p}$

$C_{1}=0.645 ; C_{2}=116 ; C_{3}=0.4 ; C_{4}=5 ; C_{5}=21 ; C_{6}=0.009 ; C_{7}=0.08 ; C_{8}=0.35 ;$

\section{DC-Link:}

$C=10000 E-6[$ Frads $]$

$V_{\text {dcnominal }}=1150[\mathrm{~V}]$

\section{Controller data:}

Rotor side converter controller:

$K_{p 1}=1.25 ; K_{i 1}=300-$ Active power loop

$K_{p 2}=0.6 ; K_{i 2}=8-$ Inner current controller loop $(d-$ axis $)$

$K_{p 3}=1.25 ; K_{i 3}=300-$ Stator Reactive power loop 
$K_{p 4}=0.6 ; K_{i 4}=8-$ Inner current controller loop $(q-$ axis $)$

Grid side converter controller:

$K_{p 5}=8 ; K_{i 5}=400-$ DC-link controller

$K_{p 6}=0.83 ; K_{i 6}=5-$ Inner current controller loop $(d-$ axis $)$

$K_{p 7}=1.25 ; K_{i 7}=300-$ Grid side Reactive power controller

$K_{p 8}=0.83 ; K_{i 8}=5-$ Inner current controller loop $(q-$ axis $)$

\section{PLL:}

$K_{p p l l}=50 ; K_{i p l l}=500$

\section{Reference values:}

$Q_{\text {sref }}=Q_{\text {gref }}=0$ Reactive power reference values

$V_{\text {dcref }}=V_{\text {dcnominal }}$

PSS with voltage as input:

$K_{p}=5 ; T_{w}=T_{1}=1$

PSS with frequency as input (transformation technique):

$K_{f}=10$

$T_{w}=2 ;$ And $T_{1}=1$

\section{Pitch controller:}

$K_{p p}=150 ; K_{p c}=3 ; K_{i c}=30$

$\theta_{\max }=27 ; \theta_{\min }=0$

Operating point values for small signal analysis:

$$
\begin{aligned}
& v_{d s 0}=V_{0} ; v_{q s 0}=0 ; \\
& i_{d s 0}=P_{s r e f} / V_{0} ; v_{q s 0}=Q_{s r e f} / V_{0} ; \\
& i_{d r 0}=\left(1 / L_{m}\right)\left(\left(R_{s} i_{q s 0}\right)+\left(L_{s s} i_{d s o}\right)\right)
\end{aligned}
$$




$$
\begin{aligned}
& i_{q r 0}=\left(1 / L_{m}\right)\left(\left(R_{s} i_{d s 0}\right)-\left(L_{s s} i_{q s o}\right)\right) \\
& i_{d 0}=V_{d c r e f} / V_{d c 0} ; i_{d 0}=Q_{r e f} / V_{0} \\
& i_{d g 0}=\left(i_{d 0}-i_{d s 0}\right) ; i_{q g 0}=\left(i_{q 0}-i_{q s 0}\right) \\
& V_{d g 0}=\left(R_{g} i_{d g 0}\right)-\left(\omega_{0} L_{g} i_{q g 0}\right)+v_{d s 0} \\
& V_{q g 0}=\left(R_{g} i_{q g 0}\right)+\left(\omega_{0} L_{g} i_{d g 0}\right)+v_{q s 0}
\end{aligned}
$$




\section{Appendix C: Large signal equations:}

Aerodynamic model:

$$
\begin{gathered}
P_{t}=0.5 \rho A V_{w}^{3} C_{p}(\lambda, \theta) \\
\lambda=\frac{\omega_{t} R}{V_{w}}
\end{gathered}
$$

Drive train model

$$
\begin{gathered}
2 H_{t} p \omega_{t}=T_{m}-D_{t} \omega_{t}-D_{t g}\left(\omega_{t}-\omega_{r}\right)-T_{t g} \\
2 H_{g} p \omega_{r}=D_{t g}\left(\omega_{t}-\omega_{r}\right)+T_{t g}-D_{g} \omega_{r}-T_{e} \\
p T_{t g}=K_{t g}\left(\omega_{t}-\omega_{r}\right) \\
T_{m}=\frac{P_{t}}{\omega_{t}}
\end{gathered}
$$

Electrical generator model in $a b c$ reference frame

$$
\begin{gathered}
v_{a s}=R_{s} i_{a s}+\frac{d \psi_{a s}}{d t} \\
v_{b s}=R_{s} i_{b s}+\frac{d \psi_{b s}}{d t} \\
v_{c s}=R_{s} i_{c s}+\frac{d \psi_{c s}}{d t} \\
v_{a r}=R_{r} i_{a r}+\frac{d \psi_{a r}}{d t} \\
v_{b r}=R_{r} i_{b r}+\frac{d \psi_{b r}}{d t} \\
v_{c r}=R_{r} i_{c r}+\frac{d \psi_{c r}}{d t} \\
\psi_{a b c s}=L_{s s} i_{a b c s}+L_{s r} i_{a b c r} \\
\psi_{a b c r}=L_{r r} i_{a b c r}+L_{r s} i_{a b c s}
\end{gathered}
$$

Electrical generator model in $d q$ reference frame 


$$
\begin{aligned}
& v_{d s}=r_{s} i_{d s}-\frac{\omega_{s}}{\omega_{b}} \psi_{q s}+p \psi_{d s} \\
& v_{q s}=r_{s} i_{q s}+\frac{\omega_{s}}{\omega_{b}} \psi_{d s}+p \psi_{q s} \\
& v_{d r}=r_{r} i_{d r}-\left(\frac{\omega_{s}-\omega_{r}}{\omega_{b}}\right) \psi_{q r}+p \psi_{d r} \\
& v_{q r}=r_{r} i_{q r}+\left(\frac{\omega_{s}-\omega_{r}}{\omega_{b}}\right) \psi_{d r}+p \psi_{q r} \\
& \psi_{d s}=L_{s} i_{d s}+L_{m} i_{d r} \\
& \psi_{q s}=L_{s} i_{q s}+L_{m} i_{q r} \\
& \psi_{d r}=L_{r} i_{d r}+L_{m} i_{d s} \\
& \psi_{q r}=L_{r} i_{q r}+L_{m} i_{q s} \\
& L_{s}=L_{l s}+L_{m} \\
& L_{r}=L_{l r}+L_{m} \\
& P_{s}=\frac{3}{2}\left(v_{d s} i_{d s}+v_{q s} i_{q s}\right) \\
& Q_{s}=\frac{3}{2}\left(v_{q s} i_{d s}-v_{d s} i_{q s}\right) \\
& P_{r}=\frac{3}{2}\left(v_{d r} i_{d r}+v_{q r} i_{q r}\right) \\
& Q_{r}=\frac{3}{2}\left(v_{q r} i_{d r}-v_{d r} i_{q r}\right) \\
& T_{e}=L_{m}\left(i_{q s} i_{d r}-i_{d s} i_{q r}\right)
\end{aligned}
$$

DC-Link model:

$$
\begin{gathered}
P_{d c}=V_{d c} I_{d c}=C V_{d c} \frac{d V_{d c}}{d t} \\
\frac{d V_{d c}}{d t}=\frac{1}{V_{d c} C}\left(v_{d r} i_{d r}+v_{q r} i_{q r}-\left(v_{d g} i_{d g}+v_{q g} i_{q g}\right)\right)
\end{gathered}
$$


The network model in $a b c$ reference frame can be expressed as:

$$
\begin{gathered}
v_{s a}=R i_{a}+L p i_{a}+e_{a} \\
v_{s b}=R i_{b}+L p i_{b}+e_{b} \\
v_{s c}=R i_{c}+L p i_{c}+e_{c} \\
i_{a}=i_{s a}+i_{g a} \\
i_{b}=i_{s b}+i_{g b} \\
i_{c}=i_{s c}+i_{g c} \\
v_{g a}=R_{g} i_{g a}+L_{g} p i_{g a}+v_{s a} \\
v_{g b}=R_{g} i_{g a}+L_{g} p i_{g a}+v_{s a} \\
v_{g c}=R_{g} i_{g a}+L_{g} p i_{g a}+v_{s a}
\end{gathered}
$$

Large signal equations of controllers:

$$
\begin{gathered}
\omega_{p l l}=\left(k_{p p l l}+\frac{k_{i p l l}}{s}\right) v_{q s}+\omega_{0} \\
p \delta_{p l l}=\omega_{p l l}-\omega_{0} \\
v_{d s}=r i_{d}+L p i_{d}-\omega_{p l l} L i_{q}+e_{d} \\
v_{q s}=r i_{q}+L p i_{q}-\omega_{p l l} L i_{d}+e_{q} \\
i_{d}=i_{d s}+i_{d g} \\
i_{q}=i_{q s}+i_{q g} \\
v_{d g}=R_{g} i_{d g}+L_{g} p i_{d g}-\omega_{p l l} L_{g} i_{q g}+v_{d s} \\
v_{q g}=R_{g} i_{q g}+L_{g} p i_{q g}+\omega_{p l l} L_{g} i_{d g}+v_{q s} \\
e_{d}=E \cos \left(\delta_{p l l}\right) \\
e_{q}=-E \sin \left(\delta_{p l l}\right)
\end{gathered}
$$




$$
\begin{gathered}
P_{g c}=\frac{3}{2}\left(v_{d s} i_{d g}+v_{q s} i_{q g}\right) \\
Q_{g}=\frac{3}{2}\left(v_{q s} i_{d g}-v_{d s} i_{q g}\right) \\
v_{d g}=R_{g} i_{d g}+L_{g} p i_{d g}-\omega_{p l l} L_{g} i_{q g}+v_{d s} \\
v_{q g}=R_{g} i_{q g}+L_{g} p i_{q g}+\omega_{p l l} L_{g} i_{d g} \\
v_{d g}=R_{g} i_{d g}+L_{g} p i_{d g} \\
v_{q g}=R_{g} i_{q g}+L_{g} p i_{q g}
\end{gathered}
$$

Rotor side controller large signal model:

$$
\begin{gathered}
p x_{1}=P_{\text {sref }}-P_{s} \\
p x_{2}=K_{p 1}\left(P_{\text {sref }}-P_{s}\right)+K_{i 1} x_{1}-i_{d r} \\
v_{d r}=K_{p 2} K_{p 1}\left(P_{s r e f}-P_{s}\right)+K_{p 2} K_{i 1} x_{1}-K_{p 2} i_{d r}+K_{i 2} x_{2}-\left(\omega_{s}-\omega_{r}\right) L_{r} i_{q r} \\
-\left(\omega_{s}-\omega_{r}\right) L_{m} i_{q s} \\
p x_{3}=Q_{s r e f}-Q_{s} \\
p x_{4}=K_{p 3}\left(Q_{s r e f}-Q_{s}\right)+K_{i 3} x_{3}-i_{q r} \\
v_{q r}=K_{p 4} K_{p 3}\left(Q_{s r e f}-Q_{s}\right)+K_{p 4} K_{i 3} x_{3}-K_{p 4} i_{q r}+K_{i 4} x_{4}+\left(\omega_{s}-\omega_{r}\right) L_{r} i_{d r} \\
-\left(\omega_{s}-\omega_{r}\right) L_{m} i_{d s}
\end{gathered}
$$

Grid side controller large signal model:

$$
\begin{gathered}
p x_{5}=V_{d c}^{*}-V_{d c} \\
p x_{6}=K_{p 5}\left(V_{d c}^{*}-V_{d c}\right)+K_{i 5} x_{5}-i_{d g} \\
v_{d g}=K_{p 6} K_{p 5}\left(V_{d c}^{*}-V_{d c}\right)+K_{p 6} K_{i 5} x_{5}-K_{p 6} i_{d g}+K_{i 6} x_{6}+L_{g}\left(\omega_{p l l}\right) i_{q g}+v_{d s} \\
p x_{7}=Q_{g}^{*}-Q_{g} \\
p x_{8}=K_{p 7}\left(Q_{g}^{*}-Q_{g}\right)+K_{i 7} x_{7}-i_{q g}
\end{gathered}
$$




$$
v_{q g}=K_{p 8} K_{p 7}\left(Q_{g}^{*}-Q_{g}\right)+K_{p 8} K_{i 7} x_{7}-K_{p 8} i_{q g}+K_{i 8} x_{8}-L_{g}\left(\omega_{p l l}\right) i_{d g}
$$




\section{Appendix D: Small signal equations:}

Aerodynamic small model:

$$
\begin{gathered}
2 H_{t} p\left(\Delta \omega_{t}\right)=-\left(\frac{-P_{t}}{\omega_{t 0}^{2}}-D_{t g}\right)\left(\Delta \omega_{t}\right)-K_{t g} \Delta\left(\theta_{t}-\theta_{r}\right)+D_{t g}\left(\Delta \omega_{r}\right) \\
p \Delta\left(\theta_{t}-\theta_{r}\right)=\left(\Delta \omega_{t}\right)-\left(\Delta \omega_{r}\right) \\
2 H_{g} p\left(\Delta \omega_{r}\right)=K_{t g} \Delta\left(\theta_{t}-\theta_{r}\right)+D_{t g}\left(\Delta \omega_{t}\right)-D_{t g}\left(\Delta \omega_{r}\right)-L_{m} i_{q s 0}\left(\Delta i_{d r}\right)-L_{m} i_{d r 0}\left(\Delta i_{q s}\right) \\
+L_{m} i_{d s 0}\left(\Delta i_{q r}\right)+L_{m} i_{q r 0}\left(\Delta i_{d s}\right)
\end{gathered}
$$

DFIG small signal model:

$$
\begin{gathered}
\frac{L_{s}}{\omega_{b}} p\left(\Delta i_{d s}\right)+\frac{L_{m}}{\omega_{b}} p\left(\Delta i_{d r}\right)=\left(\Delta v_{d s}\right)+\frac{\omega_{e}}{\omega_{b}} L_{s}\left(\Delta i_{q s}\right)-R_{s}\left(\Delta i_{d s}\right)+\frac{\omega_{e}}{\omega_{b}} L_{m}\left(\Delta i_{q r}\right) \\
\frac{L_{s}}{\omega_{b}} p\left(\Delta i_{q s}\right)+\frac{L_{m}}{\omega_{b}} p\left(\Delta i_{q r}\right)=\left(\Delta v_{q s}\right)-R_{s}\left(\Delta i_{q s}\right)-\frac{\omega_{e}}{\omega_{b}} L_{s}\left(\Delta i_{d s}\right)-\frac{\omega_{e}}{\omega_{b}} L_{m}\left(\Delta i_{d r}\right) \\
\frac{L_{r}}{\omega_{b}} p\left(\Delta i_{d r}\right)+\frac{L_{m}}{\omega_{b}} p\left(\Delta i_{d s}\right)=\left(\Delta v_{d r}\right)+b_{1}\left(\Delta i_{q s}\right)-a_{1}\left(\Delta \omega_{r}\right)+c_{1}\left(\Delta i_{q r}\right)-R_{r}\left(\Delta i_{d r}\right)
\end{gathered}
$$

Where $a_{1}=\left(\frac{L_{m}}{\omega_{b}} i_{q s 0}+\frac{L_{r}}{\omega_{b}} i_{q r 0}\right) ; b_{1}=\frac{L_{m}}{\omega_{b}}\left(\omega_{e}-\omega_{r 0}\right) c_{1}=\frac{L_{r}}{\omega_{b}}\left(\omega_{e}-\omega_{r 0}\right)$

$$
\frac{L_{r}}{\omega_{b}} p\left(\Delta i_{q r}\right)+\frac{L_{m}}{\omega_{b}} p\left(\Delta i_{q s}\right)=\left(\Delta v_{q r}\right)-b_{1}\left(\Delta i_{d s}\right)+a_{2}\left(\Delta \omega_{r}\right)-c_{1}\left(\Delta i_{d r}\right)-R_{r}\left(\Delta i_{d r}\right)
$$

Where $a_{2}=\left(\frac{L_{m}}{\omega_{b}} i_{d s 0}+\frac{L_{r}}{\omega_{b}} i_{d r 0}\right)$

DC-link small signal model:

$$
C p\left(\Delta v_{d c}\right)=a_{3}\left(\Delta v_{d c}\right)+\frac{1}{v_{d c 0}}\left(b_{2}-c_{2}\right)
$$

Where $a_{3}=\frac{1}{v_{d c 0}^{2}}\left(v_{d r 0} i_{d r 0}+v_{q r 0} i_{q r 0}-\left(v_{d g 0} i_{d g 0}+v_{q g 0} i_{q g 0}\right)\right)$;

$$
\begin{gathered}
b_{2}=\left(v_{d g 0}\left(\Delta i_{d g}\right)+i_{d g 0}\left(\Delta v_{d g}\right)+v_{q g 0}\left(\Delta i_{q g}\right)+i_{q g 0}\left(\Delta v_{q g}\right)\right) \\
c_{2}=\left(v_{d r 0}\left(\Delta i_{d r}\right)+i_{d r 0}\left(\Delta v_{d r}\right)+v_{q r 0}\left(\Delta i_{q r}\right)+i_{q r 0}\left(\Delta v_{q r}\right)\right)
\end{gathered}
$$


Controller model:

$$
\begin{gathered}
p \Delta \theta_{p l l}=\Delta \omega_{p l l} \\
p\left(\Delta \omega_{p l l}\right)-k_{p p l l} p\left(\Delta v_{q s}\right)=k_{i p l l}\left(\Delta v_{q S}\right)
\end{gathered}
$$

Linearized model of the rotor side controller:

$$
\begin{aligned}
& p\left(\Delta x_{1}\right)=-\left(v_{d s 0}\left(\Delta i_{d s}\right)+i_{d s 0}\left(\Delta v_{d s}\right)+v_{q s 0}\left(\Delta i_{q s}\right)+i_{q s 0}\left(\Delta v_{q s}\right)\right) \\
& p\left(\Delta x_{2}\right)=-K_{p 1}\left(v_{d s 0}\left(\Delta i_{d s}\right)+i_{d s 0}\left(\Delta v_{d s}\right)+v_{q s 0}\left(\Delta i_{q s}\right)+i_{q s 0}\left(\Delta v_{q s}\right)\right)+K_{i 1} \Delta x_{1} \\
& -\Delta i_{d r} \\
& \Delta v_{d r}=-K_{p 2} K_{p 1}\left(v_{d s 0}\left(\Delta i_{d s}\right)+i_{d s 0}\left(\Delta v_{d s}\right)+v_{q s 0}\left(\Delta i_{q s}\right)+i_{q s 0}\left(\Delta v_{q s}\right)\right) \\
& +\left(L_{m}\left(\omega_{r 0}-\omega_{s}\right)-K_{p 2} K_{p 1} v_{q s 0}\right)\left(\Delta i_{q s}\right)+K_{p 2} K_{i 1} \Delta x_{1}-K_{p 2} \Delta i_{d r}+K_{i 2} \Delta x_{2} \\
& -\left(\omega_{r 0}-\omega_{s}\right) L_{r} \Delta i_{q r}+L_{r}\left(i_{q r o}+i_{q s o}\right) \Delta \omega_{r} \\
& p\left(\Delta x_{3}\right)=-v_{d s 0}\left(\Delta i_{q s}\right)-i_{q s 0}\left(\Delta v_{d s}\right)+v_{q s 0}\left(\Delta i_{d s}\right)+i_{d s 0}\left(\Delta v_{q s}\right) \\
& p\left(\Delta x_{4}\right)=K_{p 3}\left(-v_{d s 0}\left(\Delta i_{q s}\right)-i_{q s 0}\left(\Delta v_{d s}\right)+v_{q s 0}\left(\Delta i_{d s}\right)+i_{d s 0}\left(\Delta v_{q s}\right)\right)+K_{i 1} \Delta x_{3} \\
& -\Delta i_{q r} \\
& \Delta v_{q r}=K_{p 4} K_{p 3}\left(-v_{d s 0}\left(\Delta i_{q s}\right)-i_{q s 0}\left(\Delta v_{d s}\right)+v_{q s 0}\left(\Delta i_{d s}\right)+i_{d s 0}\left(\Delta v_{q s}\right)\right) \\
& +\left(L_{m}\left(\omega_{s}-\omega_{r 0}\right)+K_{p 4} K_{p 3} v_{q s 0}\right)\left(\Delta i_{d s}\right)+K_{p 4} K_{i 3} \Delta x_{3}-K_{p 4} \Delta i_{q r}+K_{i 4} \Delta x_{4} \\
& +\left(\omega_{s}-\omega_{r 0}\right) L_{r} \Delta i_{d r}-L_{r}\left(i_{d r o}+i_{d s o}\right) \Delta \omega_{r}
\end{aligned}
$$

Linearized model of the grid side controller:

$$
\begin{gathered}
p \Delta x_{5}=-\Delta V_{d c} \\
p \Delta x_{6}=-K_{p 5}\left(\Delta V_{d c}\right)+K_{i 5} \Delta x_{5}-\Delta i_{d g} \\
\Delta v_{d g}=-K_{p 6} K_{p 5}\left(\Delta V_{d c}\right)+K_{p 6} K_{i 5} \Delta x_{5}-K_{p 6} \Delta i_{d g}+K_{i 6} \Delta x_{6}+L_{g}\left(\omega_{p l l 0}\right) \Delta i_{q g}+\Delta v_{d s} \\
+L_{g} i_{q g 0}\left(\Delta \omega_{p l l}\right)
\end{gathered}
$$




$$
\begin{gathered}
p \Delta x_{7}=-\Delta Q_{g} \\
p \Delta x_{8}=-K_{p 7}\left(\Delta Q_{g}\right)+K_{i 7} \Delta x_{7}-\Delta i_{q g} \\
\Delta v_{q g}=\left(-K_{p 8} K_{p 7} v_{d s 0}-K_{p 8}\right)\left(\Delta i_{q g}\right)-K_{p 8} K_{p 7} i_{q g 0}\left(\Delta v_{d s}\right)+\left(K_{p 8} K_{p 7} v_{q s 0}-L_{g} \omega_{p l l 0}\right)\left(\Delta i_{d g}\right) \\
+K_{p 8} K_{p 7} i_{d g 0}\left(\Delta v_{q s}\right)+K_{p 8} K_{i 7} \Delta x_{7}-K_{p 8} i_{q g}+K_{i 8} \Delta x_{8} \\
-L_{g} i_{d g 0}\left(\Delta \omega_{p l l}\right)
\end{gathered}
$$

Where $\Delta Q_{g}=v_{d s 0} \Delta i_{q g}+i_{q g 0} \Delta v_{d s}-\left(v_{q s 0} \Delta i_{d g}+i_{d g 0} \Delta v_{q s}\right)$

Linearized grid equations:

$$
\begin{aligned}
& L p \Delta\left(i_{d s}+i_{d g}\right) \\
& =\Delta v_{d s}-r \Delta i_{d s}-r \Delta i_{d g}+\omega_{0} L \Delta i_{q s}+\omega_{0} L \Delta i_{q g}+L\left(i_{q s 0}+i_{q g 0}\right)\left(\Delta \omega_{p l l}\right) \\
& -E \sin \delta_{0}\left(\Delta \delta_{p l l}\right)
\end{aligned}
$$

$$
\begin{aligned}
L p \Delta\left(i_{q s}+i_{q g}\right) & \\
& =\Delta v_{q s}-r \Delta i_{q s}-r \Delta i_{q g}-\omega_{0} L \Delta i_{d s}-\omega_{0} L \Delta i_{d g}-L\left(i_{d s 0}+i_{d g 0}\right)\left(\Delta \omega_{p l l}\right) \\
& -E \cos \delta_{0}\left(\Delta \delta_{p l l}\right)
\end{aligned}
$$

$$
\begin{aligned}
& L_{g} p\left(\Delta i_{d g}\right)=\left(\Delta v_{d g}\right)-R_{g}\left(\Delta i_{d g}\right)+\omega_{0} L_{g}\left(\Delta i_{q g}\right)+L_{g} i_{q g 0}\left(\Delta \omega_{p l l}\right)-\Delta v_{d s} \\
& L_{g} p\left(\Delta i_{q g}\right)=\left(\Delta v_{q g}\right)-R_{g}\left(\Delta i_{q g}\right)-\omega_{0} L_{g}\left(\Delta i_{d g}\right)-L_{g} i_{d g 0}\left(\Delta \omega_{p l l}\right)-\Delta v_{q s}
\end{aligned}
$$




\section{Appendix E: Matrices of small signal model for grid connected system}

\section{without controllers:}

Matrices $E$ and $A$ for the grid connected system without controller:

The size of matrices $E$ and $A$ are $18 \times 18$,

$$
E=\left[\begin{array}{ll}
E_{1} & E_{2}
\end{array}\right]
$$

$E_{1}$ size is $18 \times 15, E_{2}$ is $18 \times 3$

$E_{1}$

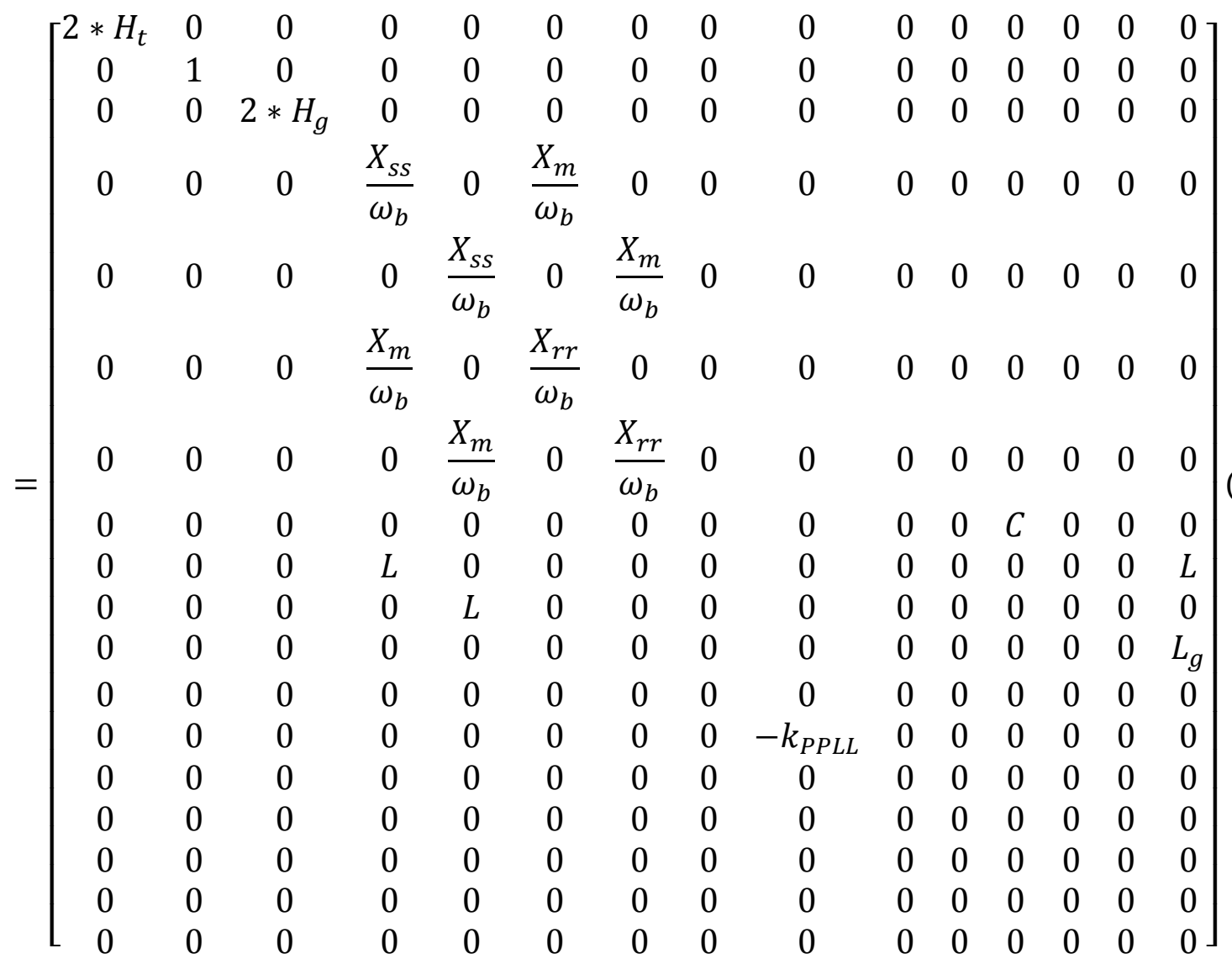




$$
\begin{gathered}
E_{2}=\left[\begin{array}{ccc}
0 & 0 & 0 \\
0 & 0 & 0 \\
0 & 0 & 0 \\
0 & 0 & 0 \\
0 & 0 & 0 \\
0 & 0 & 0 \\
0 & 0 & 0 \\
0 & 0 & 0 \\
L & 0 & 0 \\
0 & 0 & 0 \\
L_{g} & 0 & 0 \\
0 & 1 & 0 \\
0 & 0 & 1 \\
0 & 0 & 0 \\
0 & 0 & 0 \\
0 & 0 & 0 \\
0 & 0 & 0 \\
0 & 0 & 0
\end{array}\right] \\
A=\left[\begin{array}{ll}
A_{1} & A_{2}
\end{array}\right]
\end{gathered}
$$

$A_{1}$ size is $18 \times 12, E_{2}$ is $18 \times 6$

$$
A_{1}=\left[\begin{array}{cccccccccccc}
a_{11} & a_{12} & a_{13} & 0 & 0 & 0 & 0 & 0 & 0 & 0 & 0 & 0 \\
1 & 0 & -1 & 0 & 0 & 0 & 0 & 0 & 0 & 0 & 0 & 0 \\
a_{31} & a_{32} & a_{33} & a_{34} & a_{35} & a_{36} & a_{37} & 0 & 0 & 0 & 0 & 0 \\
0 & 0 & 0 & a_{44} & a_{45} & 0 & a_{47} & a_{48} & 0 & 0 & 0 & 0 \\
0 & 0 & 0 & a_{54} & a_{55} & a_{56} & 0 & 0 & a_{59} & 0 & 0 & 0 \\
0 & 0 & a_{63} & 0 & a_{65} & a_{66} & a_{67} & 0 & 0 & a_{610} & 0 & 0 \\
0 & 0 & a_{73} & a_{74} & 0 & a_{76} & a_{77} & 0 & 0 & 0 & a_{711} & 0 \\
0 & 0 & 0 & 0 & 0 & a_{86} & a_{87} & 0 & 0 & a_{810} & a_{811} & a_{812} \\
0 & 0 & 0 & a_{94} & a_{95} & 0 & 0 & a_{98} & 0 & 0 & 0 & 0 \\
0 & 0 & 0 & a_{104} & a_{105} & 0 & 0 & 0 & a_{109} & 0 & 0 & 0 \\
0 & 0 & 0 & 0 & 0 & 0 & 0 & a_{118} & 0 & 0 & 0 & 0 \\
0 & 0 & 0 & 0 & 0 & 0 & 0 & 0 & a_{129} & 0 & 0 & 0 \\
0 & 0 & 0 & 0 & 0 & 0 & 0 & 0 & k_{i P L L} & 0 & 0 & 0 \\
0 & 0 & 0 & 0 & 0 & 0 & 0 & 0 & 0 & 0 & 0 & 0 \\
0 & 0 & 0 & 0 & 0 & 0 & 0 & 0 & 0 & 0 & 0 & 0 \\
0 & 0 & 0 & 0 & 0 & 0 & 0 & 0 & 0 & 0 & 0 & 0 \\
0 & 0 & 0 & 0 & 0 & 0 & 0 & 0 & 0 & 0 & 0 & 0 \\
0 & 0 & 0 & 0 & 0 & 0 & 0 & 0 & 0 & 0 & 0 & 0
\end{array}\right]
$$




$$
A_{2}=\left[\begin{array}{cccccc}
0 & 0 & 0 & 0 & 0 & 0 \\
0 & 0 & 0 & 0 & 0 & 0 \\
0 & 0 & 0 & 0 & 0 & 0 \\
0 & 0 & 0 & 0 & 0 & 0 \\
0 & 0 & 0 & 0 & 0 & 0 \\
0 & 0 & 0 & 0 & 0 & 0 \\
0 & 0 & 0 & 0 & 0 & 0 \\
a_{813} & a_{814} & a_{815} & a_{816} & 0 & 0 \\
0 & 0 & a_{915} & a_{916} & a_{917} & a_{918} \\
0 & 0 & a_{1015} & a_{1016} & a_{1017} & a_{1018} \\
a_{1113} & 0 & a_{115} & a_{1116} & a_{1117} & 0 \\
0 & a_{1214} & a_{1215} & a_{1216} & a_{1217} & 0 \\
0 & 0 & 0 & 0 & 0 & 0 \\
0 & 0 & 0 & 0 & 1 & 0 \\
0 & 0 & 0 & 0 & 0 & 0 \\
0 & 0 & 0 & 0 & 0 & 0 \\
0 & 0 & 0 & 0 & 0 & 0 \\
0 & 0 & 0 & 0 & 0 & 0
\end{array}\right]
$$

The elements $a_{i j}$ are derived from the small signal model as shown in Appendix D for corresponding states described in Chapter 4 for the system without controllers. 


\section{Appendix F: Matrices of small signal model for grid connected system with}

\section{controllers:}

Matrices $E$ and $A$ for the grid connected system with controller:

The size of matrices $E$ and $A$ are $26 \times 26$,

$$
E=\left[\begin{array}{ll}
E_{1} & E_{2} \\
E_{3} & E_{4}
\end{array}\right]
$$

$E_{1}$ size is $18 \times 15 ; E_{2}$ is $18 \times 11 ; E_{3}$ is $8 \times 15$ and $E_{4}$ is $8 \times 11$

$$
\begin{aligned}
& E_{1} \\
& {\left[\begin{array}{ccccccccccccccc}
2 * H_{t} & 0 & 0 & 0 & 0 & 0 & 0 & 0 & 0 & 0 & 0 & 0 & 0 & 0 & 0 \\
0 & 1 & 0 & 0 & 0 & 0 & 0 & 0 & 0 & 0 & 0 & 0 & 0 & 0 & 0 \\
0 & 0 & 2 * H_{g} & 0 & 0 & 0 & 0 & 0 & 0 & 0 & 0 & 0 & 0 & 0 & 0 \\
0 & 0 & 0 & \frac{X_{s s}}{\omega_{b}} & 0 & \frac{X_{m}}{\omega_{b}} & 0 & 0 & 0 & 0 & 0 & 0 & 0 & 0 & 0
\end{array}\right]} \\
& \begin{array}{lllllllllllllll}
0 & 0 & 0 & 0 & \frac{X_{s s}}{\omega_{b}} & 0 & \frac{X_{m}}{\omega_{b}} & 0 & 0 & 0 & 0 & 0 & 0 & 0 & 0
\end{array} \\
& \begin{array}{lllllllllllllll}
0 & 0 & 0 & \frac{X_{m}}{\omega_{b}} & 0 & \frac{X_{r r}}{\omega_{b}} & 0 & 0 & 0 & 0 & 0 & 0 & 0 & 0 & 0
\end{array} \\
& =\left[\begin{array}{lllllllllllllll}
0 & 0 & 0 & 0 & \frac{X_{m}}{\omega_{b}} & 0 & \frac{X_{r r}}{\omega_{b}} & 0 & 0 & 0 & 0 & 0 & 0 & 0 & 0
\end{array}\right. \\
& \begin{array}{lllllllllllllll}
0 & 0 & 0 & 0 & 0 & 0 & 0 & 0 & 0 & 0 & 0 & C & 0 & 0 & 0
\end{array} \\
& \begin{array}{lllllllllllllll}
0 & 0 & 0 & L & 0 & 0 & 0 & 0 & 0 & 0 & 0 & 0 & 0 & 0 & L
\end{array} \\
& \begin{array}{lllllllllllllll}
0 & 0 & 0 & 0 & L & 0 & 0 & 0 & 0 & 0 & 0 & 0 & 0 & 0 & 0
\end{array} \\
& \begin{array}{lllllllllllllll}
0 & 0 & 0 & 0 & 0 & 0 & 0 & 0 & 0 & 0 & 0 & 0 & 0 & 0 & L_{g}
\end{array} \\
& \begin{array}{lllllllllllllll}
0 & 0 & 0 & 0 & 0 & 0 & 0 & 0 & 0 & 0 & 0 & 0 & 0 & 0 & 0
\end{array} \\
& \begin{array}{lllllllllllllll}
0 & 0 & 0 & 0 & 0 & 0 & 0 & 0 & -k_{P P L L} & 0 & 0 & 0 & 0 & 0 & 0
\end{array} \\
& \begin{array}{lllllllllllllll}
0 & 0 & 0 & 0 & 0 & 0 & 0 & 0 & 0 & 0 & 0 & 0 & 0 & 0 & 0
\end{array} \\
& \begin{array}{lllllllllllllll}
0 & 0 & 0 & 0 & 0 & 0 & 0 & 0 & 0 & 0 & 0 & 0 & 0 & 0 & 0
\end{array} \\
& \begin{array}{lllllllllllllll}
0 & 0 & 0 & 0 & 0 & 0 & 0 & 0 & 0 & 0 & 0 & 0 & 0 & 0 & 0
\end{array} \\
& \left.\begin{array}{lllllllllllllll}
0 & 0 & 0 & 0 & 0 & 0 & 0 & 0 & 0 & 0 & 0 & 0 & 0 & 0 & 0 \\
0 & 0 & 0 & 0 & 0 & 0 & 0 & 0 & 0 & 0 & 0 & 0 & 0 & 0 & 0
\end{array}\right]
\end{aligned}
$$




$$
\begin{aligned}
& E_{2}=\left[\begin{array}{lllllllllll}
0 & 0 & 0 & 0 & 0 & 0 & 0 & 0 & 0 & 0 & 0 \\
0 & 0 & 0 & 0 & 0 & 0 & 0 & 0 & 0 & 0 & 0 \\
0 & 0 & 0 & 0 & 0 & 0 & 0 & 0 & 0 & 0 & 0 \\
0 & 0 & 0 & 0 & 0 & 0 & 0 & 0 & 0 & 0 & 0 \\
0 & 0 & 0 & 0 & 0 & 0 & 0 & 0 & 0 & 0 & 0 \\
0 & 0 & 0 & 0 & 0 & 0 & 0 & 0 & 0 & 0 & 0 \\
0 & 0 & 0 & 0 & 0 & 0 & 0 & 0 & 0 & 0 & 0 \\
0 & 0 & 0 & 0 & 0 & 0 & 0 & 0 & 0 & 0 & 0 \\
0 & 0 & 0 & 0 & 0 & 0 & 0 & 0 & 0 & 0 & 0 \\
L & 0 & 0 & 0 & 0 & 0 & 0 & 0 & 0 & 0 & 0 \\
0 & 0 & 0 & 0 & 0 & 0 & 0 & 0 & 0 & 0 & 0 \\
L_{g} & 0 & 0 & 0 & 0 & 0 & 0 & 0 & 0 & 0 & 0 \\
0 & 0 & 1 & 0 & 0 & 0 & 0 & 0 & 0 & 0 & 0 \\
0 & 0 & 0 & 1 & 0 & 0 & 0 & 0 & 0 & 0 & 0 \\
0 & 0 & 0 & 0 & 1 & 0 & 0 & 0 & 0 & 0 & 0 \\
0 & 0 & 0 & 0 & 0 & 1 & 0 & 0 & 0 & 0 & 0 \\
0 & 0 & 0 & 0 & 0 & 0 & 1 & 0 & 0 & 0 & 0 \\
0 & 0 & 0 & 0 & 0 & 0 & 0 & 1 & 0 & 0 & 0
\end{array}\right] \\
& E_{3}=\left[\begin{array}{lllllllllllllll}
0 & 0 & 0 & 0 & 0 & 0 & 0 & 0 & 0 & 0 & 0 & 0 & 0 & 0 & 0 \\
0 & 0 & 0 & 0 & 0 & 0 & 0 & 0 & 0 & 0 & 0 & 0 & 0 & 0 & 0 \\
0 & 0 & 0 & 0 & 0 & 0 & 0 & 0 & 0 & 0 & 0 & 0 & 0 & 0 & 0 \\
0 & 0 & 0 & 0 & 0 & 0 & 0 & 0 & 0 & 0 & 0 & 0 & 0 & 0 & 0 \\
0 & 0 & 0 & 0 & 0 & 0 & 0 & 0 & 0 & 0 & 0 & 0 & 0 & 0 & 0 \\
0 & 0 & 0 & 0 & 0 & 0 & 0 & 0 & 0 & 0 & 0 & 0 & 0 & 0 & 0 \\
0 & 0 & 0 & 0 & 0 & 0 & 0 & 0 & 0 & 0 & 0 & 0 & 0 & 0 & 0 \\
0 & 0 & 0 & 0 & 0 & 0 & 0 & 0 & 0 & 0 & 0 & 0 & 0 & 0 & 0
\end{array}\right] \\
& E_{4}=\left[\begin{array}{lllllllllll}
0 & 0 & 0 & 0 & 0 & 0 & 0 & 1 & 0 & 0 & 0 \\
0 & 0 & 0 & 0 & 0 & 0 & 0 & 0 & 1 & 0 & 0 \\
0 & 0 & 0 & 0 & 0 & 0 & 0 & 0 & 0 & 1 & 0 \\
0 & 0 & 0 & 0 & 0 & 0 & 0 & 0 & 0 & 0 & 1 \\
0 & 0 & 0 & 0 & 0 & 0 & 0 & 0 & 0 & 0 & 0 \\
0 & 0 & 0 & 0 & 0 & 0 & 0 & 0 & 0 & 0 & 0 \\
0 & 0 & 0 & 0 & 0 & 0 & 0 & 0 & 0 & 0 & 0 \\
0 & 0 & 0 & 0 & 0 & 0 & 0 & 0 & 0 & 0 & 0
\end{array}\right] \\
& A=\left[\begin{array}{ll}
A_{1} & A_{2} \\
A_{3} & A_{4}
\end{array}\right]
\end{aligned}
$$

$A_{1}$ size is $18 \times 12 ; A_{2}$ is $18 \times 14 ; A_{3}$ is $8 \times 12$ and $A_{4}$ is $8 \times 14$ 


$$
\begin{aligned}
& A_{1}=\left[\begin{array}{cccccccccccc}
a_{11} & a_{12} & a_{13} & 0 & 0 & 0 & 0 & 0 & 0 & 0 & 0 & 0 \\
1 & 0 & -1 & 0 & 0 & 0 & 0 & 0 & 0 & 0 & 0 & 0 \\
a_{31} & a_{32} & a_{33} & a_{34} & a_{35} & a_{36} & a_{37} & 0 & 0 & 0 & 0 & 0 \\
0 & 0 & 0 & a_{44} & a_{45} & 0 & a_{47} & a_{48} & 0 & 0 & 0 & 0 \\
0 & 0 & 0 & a_{54} & a_{55} & a_{56} & 0 & 0 & a_{59} & 0 & 0 & 0 \\
0 & 0 & a_{63} & 0 & a_{65} & a_{66} & a_{67} & 0 & 0 & a_{610} & 0 & 0 \\
0 & 0 & a_{73} & a_{74} & 0 & a_{76} & a_{77} & 0 & 0 & 0 & a_{711} & 0 \\
0 & 0 & 0 & 0 & 0 & a_{86} & a_{87} & 0 & 0 & a_{810} & a_{811} & a_{812} \\
0 & 0 & 0 & a_{94} & a_{95} & 0 & 0 & a_{98} & 0 & 0 & 0 & 0 \\
0 & 0 & 0 & a_{104} & a_{105} & 0 & 0 & 0 & a_{109} & 0 & 0 & 0 \\
0 & 0 & 0 & 0 & 0 & 0 & 0 & a_{118} & 0 & 0 & 0 & 0 \\
0 & 0 & 0 & 0 & 0 & 0 & 0 & 0 & a_{129} & 0 & 0 & 0 \\
0 & 0 & 0 & 0 & 0 & 0 & 0 & 0 & k_{i P L L} & 0 & 0 & 0 \\
0 & 0 & 0 & 0 & 0 & 0 & 0 & 0 & 0 & 0 & 0 & 0 \\
0 & 0 & 0 & a_{154} & a_{155} & 0 & 0 & a_{158} & a_{159} & 0 & 0 & 0 \\
0 & 0 & 0 & a_{164} & a_{165} & 0 & -1 & a_{168} & a_{169} & 0 & 0 & 0 \\
0 & 0 & 0 & a_{174} & a_{175} & 0 & 0 & a_{178} & a_{179} & 0 & 0 & 0 \\
0 & 0 & 0 & a_{184} & a_{185} & -1 & 0 & a_{188} & a_{189} & 0 & 0 & 0
\end{array}\right](F .7) \\
& A_{2} \\
& {\left[\begin{array}{cccccccccccccc}
0 & 0 & 0 & 0 & 0 & 0 & 0 & 0 & 0 & 0 & 0 & 0 & 0 & 0 \\
0 & 0 & 0 & 0 & 0 & 0 & 0 & 0 & 0 & 0 & 0 & 0 & 0 & 0 \\
0 & 0 & 0 & 0 & 0 & 0 & 0 & 0 & 0 & 0 & 0 & 0 & 0 & 0 \\
0 & 0 & 0 & 0 & 0 & 0 & 0 & 0 & 0 & 0 & 0 & 0 & 0 & 0 \\
0 & 0 & 0 & 0 & 0 & 0 & 0 & 0 & 0 & 0 & 0 & 0 & 0 & 0 \\
0 & 0 & 0 & 0 & 0 & 0 & 0 & 0 & 0 & 0 & 0 & 0 & 0 & 0 \\
0 & 0 & 0 & 0 & 0 & 0 & 0 & 0 & 0 & 0 & 0 & 0 & 0 & 0 \\
a_{813} & a_{814} & a_{815} & a_{816} & 0 & 0 & 0 & 0 & 0 & 0 & 0 & 0 & 0 & 0 \\
0 & 0 & a_{915} & a_{916} & a_{917} & a_{918} & 0 & 0 & 0 & 0 & 0 & 0 & 0 & 0 \\
0 & 0 & a_{1015} & a_{1016} & a_{1017} & a_{1018} & 0 & 0 & 0 & 0 & 0 & 0 & 0 & 0 \\
a_{1113} & 0 & a_{1115} & a_{1116} & a_{1117} & 0 & 0 & 0 & 0 & 0 & 0 & 0 & 0 & 0 \\
0 & a_{1214} & a_{1215} & a_{1216} & a_{1217} & 0 & 0 & 0 & 0 & 0 & 0 & 0 & 0 & 0 \\
0 & 0 & 0 & 0 & 0 & 0 & 0 & 0 & 0 & 0 & 0 & 0 & 0 & 0 \\
0 & 0 & 0 & 0 & 1 & 0 & 0 & 0 & 0 & 0 & 0 & 0 & 0 & 0 \\
0 & 0 & 0 & 0 & 0 & 0 & 0 & 0 & 0 & 0 & 0 & 0 & 0 & 0 \\
0 & 0 & 0 & 0 & 0 & 0 & a_{1619} & 0 & 0 & 0 & 0 & 0 & 0 & 0 \\
0 & 0 & 0 & 0 & 0 & 0 & 0 & 0 & 0 & 0 & 0 & 0 & 0 & 0 \\
0 & 0 & 0 & 0 & 0 & 0 & 0 & 0 & a_{1821} & 0 & 0 & 0 & 0 & 0
\end{array}\right]}
\end{aligned}
$$




$$
A_{3}=\left[\begin{array}{cccccccccccc}
0 & 0 & 0 & 0 & 0 & 0 & 0 & 0 & 0 & 0 & 0 & -1 \\
0 & 0 & 0 & 0 & 0 & 0 & 0 & 0 & 0 & 0 & 0 & a_{1912} \\
0 & 0 & 0 & 0 & 0 & 0 & 0 & a_{218} & a_{219} & 0 & 0 & 0 \\
0 & 0 & 0 & 0 & 0 & 0 & 0 & a_{228} & a_{229} & 0 & 0 & 0 \\
0 & 0 & a_{233} & a_{234} & a_{235} & a_{236} & a_{237} & a_{238} & a_{239} & 0 & -1 & 0 \\
0 & 0 & a_{243} & a_{244} & a_{245} & a_{246} & a_{247} & a_{248} & a_{249} & -1 & 0 & 0 \\
0 & 0 & 0 & 0 & 0 & 0 & 0 & a_{258} & 0 & 0 & 0 & a_{2512} \\
0 & 0 & 0 & 0 & 0 & 0 & 0 & a_{268} & a_{269} & 0 & 0 & 0
\end{array}\right]
$$

$$
=\left[\begin{array}{cccccccccccccc}
0 & \multicolumn{10}{c}{A_{4}} \\
0 & 0 & 0 & 0 & 0 & 0 & 0 & 0 & 0 & 0 & 0 & 0 & 0 & 0 \\
0 & 0 & a_{2115} & a_{2116} & 0 & 0 & 0 & 0 & 0 & 0 & 0 & 0 & 0 & 0 \\
0 & 0 & a_{2215} & a_{2216} & 0 & 0 & 0 & 0 & 0 & 0 & 0 & 0 & a_{2225} & 0 \\
0 & 0 & 0 & 0 & 0 & 0 & a_{2319} & k_{i 2} & 0 & 0 & 0 & 0 & 0 & 0 \\
0 & 0 & 0 & 0 & 0 & 0 & 0 & 0 & a_{2421} & k_{i 4} & 0 & 0 & 0 & 0 \\
-1 & 0 & a_{2515} & a_{2516} & a_{2517} & 0 & 0 & 0 & 0 & 0 & a_{2523} & k_{i 6} & 0 & 0 \\
0 & -1 & a_{2615} & a_{2616} & a_{2616} & 0 & 0 & 0 & 0 & 0 & 0 & 0 & a_{2625} & k_{i 8}
\end{array}\right]
$$

The elements $a_{i j}$ are derived from the small signal model as shown in Appendix D for corresponding states described in Chapter 4 for the system with controllers.

Simailr procedure is carried out for the system with the power system stabilizers. 


\section{Bibliography:}

[1] Arthouros Zervos. Renewables 2014 Global Status Report. Paris: REN21, 2014, pp 24-26

[2] NRCan, "Renewable energy," in Energy Markets Fact Book, Ed. Natural Resources Canada, 2014- 2015, pp 66-79.

[3] NRCan, "Natural Resources Canada (2014) briefing on renewable energy resources". [Online]. Available: $\quad$ http://www.nrcan.gc.ca/energy/renewable-electricity/7295\#key, April.11, 2014[Aug.8, 2014].

[4] Aradhya Sambhu Satpathy, N.K.Kishore, Debaprasad Kastha and N.C.Sahoo, "Control scheme for a stand-alone wind energy conversion system," IEEE Trans. Energy Convers., vol.29, no.2, pp. 418-425, Jun 2014.

[5] Rion Takahashi and Junji Tamura, "Frequency control of isolated power system with wind farm by using flywheel energy storage system," in Proc. int. Conf. on Electric Machines, 2008, pp. 1-6.

[6] Wei Xian, Wijia Yuan, Yu Yan and T.A.Coombs, "Minimize frequency fluctuations of isolated power system with wind farm by using superconducting magnetic energy storage," in Proc. Int. Conf. on Power Electronics and Drive Systems, Nov 2009, pp. 1329-1332.

[7] S.Sharma and B.Singh, "Control of permanent magnet synchronous generator-based standalone wind energy conversion system," IET Power Electron., vol. 5, Iss.8, pp.1519-1526, May 2012.

[8] F.Blaabjerg, F.Iov, Z.Chen and K.Ma, "Power electronics and controls for wind turbine systems," in Proc. IEEE Int. Energy Conf. Exhib (Energycon), Dec 2010, pp. 333-344. 
[9] Salman K. Salman and L.J.Teo, "Windmill modeling consideration and factors influencing the stability of a grid-connected wind power-based embedded generator," IEEE Trans. Power Syst., vol.18, no.2, pp. 793-802, May 2003.

[10] Dale S.L.Dolan and Peter W.Lehn, "Simulation model of wind turbine 3p torque oscillations due to wind shear and tower shadow," IEEE Trans. Energy Convers., vol.21, no.3, pp. 717-724, Sep 2006.

[11] Christian Eisenhut, Florian Krug, Christian Schram and Berd Klockl, "Wind- turbine model for system simulations near cut-in wind speed," IEEE Trans. Energy Convers., vol.22, no.2, pp. 414-420, Jun 2007.

[12] Scott J.Schreck and Michael C.Robinson, "Horizontal axis wind turbine blade aerodynamics in experiments and modeling," IEEE Trans. Energy Convers., vol.22, no.1, pp. 61-70, Mar 2007.

[13] J.G.Slootweg, H.Polinder and W.L.Kling, "Dynamic modelling of a wind turbine with doubly fed induction generator," in Proc. IEEE Power Eng. Soc. Summer Meeting., vol.1, 2001, pp. 644-649.

[14] H.Geng and G.Yang, "Robust pitch controller for output power levelling of variablespeed variable-pitch wind turbine generator systems," IET Renewable Power Gen., vol. 3, Iss.2, pp.168-179, Jul 2008.

[15] Yunqian Zhang, Zhe Chen, Weihao $\mathrm{Hu}$ and Ming Cheng, "Flicker mitigation by individual pitch control of variable speed wind turbines with dfig," IEEE Trans. Energy Convers., vol.29, no.1, pp. 20-28, Mar 2014.

[16] Hazim Namik and Karl Stol, "Individual blade pitch control of a spar-buoy floating wind turbine," IEEE Trans. Control Syst. Technol., vol.22, no.1, pp. 214-223, Jan 2014. 
[17] Shuai Xiao, Hua Geng, Geng Yang, "Non-linear pitch control of wind turbines for tower load reduction," IET Renewable Power Gen., vol. 8, Iss.7, pp.786-794, Feb 2014.

[18] F.D. Bianchi, H.De Battista and R.J. Mantz, "Optimal gain-scheduled control of fixedspeed active stall wind turbines," IET Renewable Power Gen., vol. 2, Iss.2, pp.228-238, Apr 2008.

[19] E.B. Muhando, T. Senjyu, A. Yona, H. Kinjo and T. Funabashi, "Disturbance rejection by dual pitch control and self-tuning regulator for wind turbine generator parametric uncertainty compensation," IET Control Theory Appl., vol. 1, Iss.5, pp.1431-1440, Feb 2007. [20] Ahmet Arda Ozdemir, Peter Seiler and Gary J. Balas, "Design tradeoffs of wind turbine preview control," IEEE Trans. Control Syst. Technol., vol.21, no.4, pp. 1143-1154, Jul 2013. [21] Shuhui Li, Timothy A. Haskew, Richard P. Swatloski and William Gathings, "Optimal and direct-current vector control of direct-driven PMSG wind turbines," IEEE Trans. Power Electron., vol.27, no.5, pp. 2325-2337, May 2012.

[22] Jingya Dai, Dewei (David) Xu, and Bin Wu, "A novel control scheme for current-sourceconverter-based PMSG wind energy conversion systems," IEEE Trans. Power Electron., vol.24, no.4, pp. 963-972, Apr 2009.

[23] Venkata Yaramasu, and Bin Wu, "Predictive control of a three-level boost converter and an NPC inverter for high-power PMSG-based medium voltage wind energy conversion systems," IEEE Trans. Power Electron.,vol.29, no.10, pp. 5308-5322, Oct 2014.

[24] Hossein Hojabri, Hossein Mokhtari, and Liuchen Chang, "Reactive power control of permanent-magnet synchronous wind generator with matrix converter," IEEE Trans. Power Del., vol.28, no.2, pp. 575-584, Apr 2013. 
[25] Mats Wang-Hansen, Robert Josefsson, and Haris Mehmedovic, "Frequency controlling wind power modeling of control strategies," IEEE Trans. Sustain. Energy, vol.4, no.4, pp. 954959, Oct 2013.

[26] W. L. CHEN and Y. Y. HSU, "Unified voltage and pitch angle controller for wind-driven induction generator system," IEEE Trans. Aerosp. Electron. Syst, vol.44, no.3, pp. 913-926, Jul 2008.

[27] R. Pena, J.C.Clare and G. M. As her, "Doubly fed induction generator using back-to-back PWM converters and its application to variable-speed wind-energy generation," in Proc. IEEE., Electr. Power Appl., vol.143, no.3, May 1996, pp. 231-241.

[28] Ted Brekken and Ned Mohan, “A novel doubly-fed induction wind generator control scheme for reactive power control and torque pulsation compensation under unbalanced grid voltage conditions," in Proc. Power Electronics Specialist Conf., vol.2, Jun 2003, pp. 760764.

[29] E. Tremblay, A. Chandra, and P.J. Lagacé, "Grid-side converter control of dfig wind turbines to enhance power quality of distribution network," in in Proc. IEEE Power Eng. Soc. General Meeting., 2006, pp. 1-6.

[30] Liyan Qu, and Wei Qiao, "Constant power control of dfig wind turbines with supercapacitor energy storage,” IEEE Trans. Ind. Appl., vol.47, no.1, pp. 359-367, Feb 2011. [31] Yu Zou, Malik E. Elbuluk, and Yilmaz Sozer, "Simulation comparisons and implementation of induction generator wind power systems," IEEE Trans. Ind. Appl., vol.49, no.3, pp. 1119-1128, Jun 2013. 
[32] B. Wu, Y. Lang, N. Zargari, and S. Kouro, Power Conversion and Control of Wind Energy Systems. John Wiley \& Sons, Inc., 2011.

[33] Tao Zhang, Liuxue Sun, and Yin Zhang, "Study on switching overvoltage in off-shore wind farms," IEEE Trans. Appl. Supercond., vol.24, no.5, Oct 2014.

[34] Tore Bakka, Hamid Reza Karimi, and Soren Christiansen, "Linear parameter-varying modelling and control of an offshore wind turbine with constrained information," IET Control theory and Applications, vol. 8, Iss.1, pp.22-29, Sep 2013.

[35] Hassan Bevrani, Masayuki Watanabe and Yasunori Mitani, Power System Monitoring and Control. NY, John Wiley \& Sons, Inc. 2014.

[36] Gregory S. Vassell, “Notheast Blackout of 1965," IEEE Power Engineering Review, Jan 1991, pp. 4-8.

[37] O. Anaya-Lara, F. M. Hughes, N. Jenkins and G. Strbac, "Power system stabiliser for a generic dfig-based wind turbine controller," in Proc. 8th IEE AC/DC Conf., Mar 2006, pp. 145-149.

[38] F. Michael Hughes, Olimpo Anaya-Lara, Nicholas Jenkins, and Goran Strbac, “Control of DFIG-based wind generation for power network support," IEEE Trans. Power Syst., vol.20, no.4, pp. 1958-1966, Nov 2005.

[39] F. Michael Hughes, Olimpo Anaya-Lara, Nicholas Jenkins, and Goran Strbac, "A power system stabilizer for DFIG-based wind generation," IEEE Trans. Power Syst., vol.21, no.2, pp. 763-772, May 2006.

[40] F. Michael Hughes, Olimpo Anaya-Lara, G. Ramtharan, Nicholas Jenkins, and Goran Strbac, "Influence of tower shadow and wind turbulence on the performance of power system 
stabilizers for DFIG-based wind farms," IEEE Trans. Energy Convers., vol.23, no.2, pp. 519528, Jun 2008.

[41] Yateendra Mishra, S. Mishra, Fangxing Li, Zhao Yang Dong, and Ramesh C. Bansal, "Small-signal stability analysis of a dfig-based wind power system under different modes of operation," IEEE Trans. Energy Convers., vol.24, no.4, pp. 972-982, Dec 2009.

[42] Y. Mishra, S. Mishra, M. Tripathy, N. Senroy, and Z. Y. Dong, "Improving stability of a DFIG-based wind power system with tuned damping controller," IEEE Trans. Energy Convers., vol.24, no.3, pp. 650-660, Sep 2009.

[43] Angelo Mendonca, and J. A. Pecas Lopes, "Simultaneous tuning of power system stabilizers installed in DFIG-based wind generation," in Proc. IEEE Power Tech., Lausanne, Switzerland, Jul 2007, pp. 219-224

[44] Katherine Elkington, Mehrdad Ghandhari and Lennart Soder, "Using power system stabilisers in doubly fed induction generators," Australasian Universities Power Engineering Conference, 2008, pp. 1-6.

[45] D. P. Ke, C. Y. Chung and Yusheng Xue, "Controller design for dfig-based wind power generation to damp inter-area oscillation," Int. Conf. on Critical Infrastructure, Sept. 2010, pp. 1-6.

[46] Bing Gong, Dewei Xu and Bin Wu, "Network damping capability of DFIG-based wind farm," in IEEE Energy conversion congress and exposition, Sept.2010, pp. 4083-4090.

[47] Durga Gautam, Vijay Vittal, Raja Ayyanar and Terry Harbour, "Supplementary control for damping power oscillations due to increased penetration of doubly fed induction generators in large power systems," in IEEE power systems conference and exposition, March.2011, pp. $1-6$. 
[48] Tossaporn Surinkaew and Issarachai Ngamroo, "Coordinated robust control of dfig wind turbine and PSS for stabilization of power oscillations considering system uncertainties," IEEE Trans. Sustain. Energy., vol.5, no.3, pp. 823-833, July 2014.

[49] Iswadi HR, D. John Morrow, Robert J. Best, "Small signal stability performance of power system during high penetration of wind generation," International Universities Power Engineering conference, Sept. 2014, pp. 1-6.

[50] Xiaoyu Wang, Walmir Freitas, Venkata Dinavahi, and Wilsun Xu, "Investigation of positive feedback anti-islanding control for multiple inverter-based distributed generators," IEEE Trans. Power Syst., vol.24, no.2, pp. 785-795, May 2009.

[51] Xiaoyu Wang, “Investigation of positive feedback anti-islanding scheme for inverterbased distributed generation” Ph.D. thesis, University of Alberta, Canada, Jun 2008.

[52] Eric Hau. Wind Turbines Fundamentals, Technologies, Applications, Economics. Springer, 2005.

[53] Dale E. Berg. "Wind Energy Conversion," in Handbook of Energy Efficiency and Renewable Energy, Frank Kreith and D.Yogi Goswami, Ed. New York: CRC Press, Taylors \&Francis Group, 2007, pp. 22-4.

[54] G. Abad, J. Lopez, M. A. Rodriguez, L. Marroyo, and G. Iwanski, Doubly Fed Induction Machine: Modeling and Control for Wind Energy Generation, John Wiley\&Sons, Inc, 2011

[55] Donald S. Zinger, and Eduard Muljadi, “Annualized wind energy improvement using variable speeds," IEEE Trans. Ind. Appl., vol.33, no.6, pp. 1444-1447, Nov 1997.

[56] Abraham Ellis and Eduard Muljadi, "Wind power plant representation in large-scale power flow simulations in WECC," in Proc. 2008 IEEE PES General Meeting., July 2008, pp. $1-6$. 
[57] Ahmed F.Zobaa, Ramesh Bansal. Handbook of Renewable Energy technology. Singapore: World Scientific Publishing Co.Pte.Ltd, 2011.

[58] Frede Blaabjerg, Zhe Chen and Soeren Baekhoej Kjaer, "Power electronics as efficient interface in dispersed power generation systems," IEEE Trans. Power Syst., vol.19, no.5, pp. 1184-1194, Sept 2004.

[59] C. V. Nayar, S. M. Islam,H. Dehbonei, K. Tan and H. Sharma. "Power Electronics for Renewable Energy Sources," in Power Electronics Handbook, 3rd ed., M.H.Rashid, Ed. Elsevier, 2011, pp.754

[60] Torbjom Thiringer, Andreas Petersson and TomPS Petru, "Grid disturbance response of wind turbines equipped with induction generator and doubly-fed induction generator," in Proc. IEEE Power Eng. Soc. Gen. Meeting, July 2003, pp. 1542-1547

[61] J. G. Slootweg, H. Polinder, and W. L. Kling, "Representing wind turbine electrical generating systems in fundamental frequency simulations," IEEE Trans. Energy Convers., vol.18, no.4, pp. 516-524, Dec 2003.

[62] Anca D. Hansen, Lars H. Hansen, "Market penetration of wind turbine concepts over the years,"

[63] Wei Qiao, "Dynamic modeling and control of doubly fed induction generators driven by wind turbines," in Proc. 2009 IEEE/PES Power Systems Conference and Exposition, March 2009, pp. 1-8.

[64] T. Ackermann "Historical Development and Current Status of Wind Power," in Wind Power in Power Systems, 2nd ed., Thomas Ackermann, Ed. John Wiley\&Sons, Inc, 2012.

[65] J. G. Slootweg, H. Polinder, and W. L. Kling, "Representing wind turbine electrical generating systems in fundamental frequency simulations," IEEE Trans. Energy Convers., vol.18, no.4, pp. 516-524, Dec 2003. 
[66] Francoise Mei, Bikash C. Pal, "Modelling of doubly-fed induction generator for power system stability study," in Proc. IEEE Power \& Energy General Meeting, July 2008, pp. 1-8. [67] J. G. Slootweg, S. W. H. de Haan, H. Polinder and W. L. Kling, "General model for representing variable speed wind turbines in power system dynamics simulations," IEEE Trans. Power Syst., vol.18, no.1, pp. 144-151, Feb 2003.

[68] N. W. Miller, W. W. Price, J. J. Sanchez-Gasca, "Dynamic modeling of GE 1.5 and 3.6 wind turbine-generators", GE-Power Systems Energy Consulting, Version 3.0, Oct. 2003.

[69] Lok-Fu Pak and Venkata Dinavahi, "Real-time simulation of a wind energy system based on the doubly-fed induction generator," IEEE Trans. Power Syst., vol.24, no.3, pp. 1301-1309, Aug 2009.

[70] Tony Burton, Nick Jenkins, David Sharpe and Ervin Bossamyi. Wind Energy Handbook. 2nd ed., John Wiley\&Sons, Inc, 2011.

[71] Hermann-Josef Wagner and Jyotirmay Mathur. Introduction to wind enengy systems, basics, technology and operation. $2^{\text {nd }}$ ed., Springer 2013

[72] Matlab. Wind turbine model of simpowersystem.

[73] By B. Wu, Y. Lang, N. Zargari, and S. Kouro, "Fundamentals Of Wind Energy Conversion System Control" in Power Conversion and Control of Wind Energy Systems, John Wiley \& Sons, Inc., 2011.

[74] J.F.Manwell, J.G.Mcgowan and A.L.Rogers, Wind Energy Explained, theory, design and applications. John Wiley \& Sons Ltd 2009.

[75] Singiresu S.Rao. Mechanical Vibrations. Prentice Hall 2011.

[76] Francoise Mei and Bikash C. Pal, "Modelling and small-signal analysis of a grid connected doubly-fed induction generator," in Proc. IEEE Power \& Energy General Meeting, June 2005, pp. 2101-2108. 
[77] Francoise Mei and Bikash C. Pal, "Modal analysis of grid-connected doubly fed induction generators," IEEE Trans. Energy Convers., vol.22, no.3, pp. 728-736, Sept 2007.

[78] Marcus V. A. Nunes, J. A. Peças Lopes, Hans Helmut Zürn, Ubiratan H. Bezerra, and Rogério G. Almeida, "Influence of the variable-speed wind generators in transient stability margin of the conventional generators integrated in electrical grids," IEEE Trans. Energy Convers., vol.19, no.4, pp. 692-701, Dec 2004.

[79] E. N. Hinrichsen and P. J. Nolan, "Dynamics and stability of wind turbine generators," IEEE Trans. Power App. Syst., vol. PAS-101, no.8, pp. 2640-2648, Aug 1982.

[80] S.K.Salman, A.L.J.Teo and I.M.Rida, "The effect of shaft modelling on the assessment of fault cct and the power quality of a wind farm," in Proc. Int. Conf. Harmonics and Quality of power, Oct 2000, pp. 994-998.

[81] R. Pena, J. C. Clare, and G. M. Asher, "A doubly fed induction generator using back-toback PWM converters supplying an isolated load from a variable speed wind turbine," in Proc. IEEE. Power Appl., pp. 380-387, Sept 1996.

[82] P. Kundur, Power System Stability and Control. McGraw-Hill, 1994.

[83] Pablo Ledesma and Julio Usaola, "Doubly fed induction generator model for transient stability analysis," IEEE Trans. Energy Convers., vol. 20, no.2, pp. 388-397, Jun 2005.

[84] Paul C. Krause, Analysis of Electric Machinery. McGraw-Hill, 1986.

[85] Paul C. Krause and C.H.Thomas, "Simulation of symmetrical induction machinery," IEEE Trans. Power App. Syst., vol. PAS-84, no.11, pp. 1038-1053, Nov 1965.

[86] Chee-Mun Ong, Dynamic Simulation of Electric Machinery using Matlab/Simulink. Prentice Hall, 1998. 
[87] Janaka B. Ekanayake, Lee Holdsworth, XueGuang Wu, and Nicholas Jenkins, “Dynamic modeling of doubly fed induction generator wind turbines," IEEE Trans. Power Syst., vol. 18, no.2, pp. 803-809, May 2003.

[88] Julio Usaola and Pablo Ledesma, "Dynamic incidence of wind turbines in networks with high wind penetration" in Proc. IEEE Power Engineering Society Summer Meeting,, Jul 2011, pp. $755-760$.

[89] Vijay Vittal and Raja Ayyanar, Grid Integration and Dynamic Impact of Wind Energy. New York: Springer, 2013.

[90] John Lygeros and Federico A. Ramponi.Class Lecture, Topic: "Lecture Notes on Linear System Theory.” Automation Control Laboratory, ETH Zurich, Zurich, Switzerland, Jan. 3, 2015.

[91] D.G.Luenberger, “Time-invariant descriptor systems,” Automatica, vol. 14, pp. 473-480, Sept. 1978. 